A Dissertation

entitled

\title{
THREE-DIMENSIONAL NUMERICAL SIMULATION AND PERFORMANCE STUDY OF AN INDUSTRIAL HELICAL STATIC MIXER
}

by

Ramin Khosravi Rahmani

Submitted as partial fulfillment of the requirements for the Doctor of Philosopby in Engineering Science

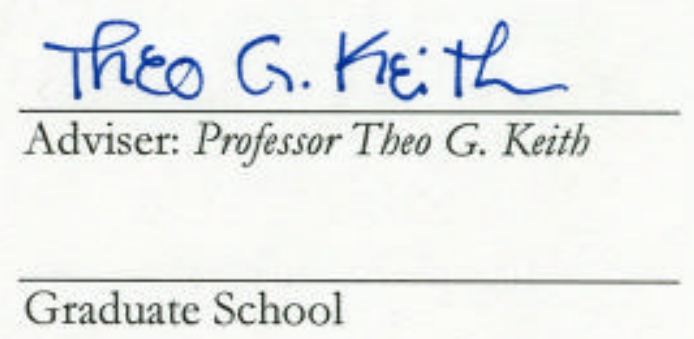

The University of Toledo

December 2004 
An Abstract of

THREE-DIMENSIONAL NUMERICAL SIMULATION AND

PERFORMANCE STUDY OF AN INDUSTRIAL HELICAL STATIC MIXER

by

Ramin Khosravi Rahmani

Submitted as partial fulfillment of the requirements for the Doctor of Philosophy in Engineering Science

The University of Toledo

December 2004

In chemical processing industries, heating, cooling and other thermal processing of viscous fluids are an integral part of the unit operations. Consequences of improper mixing include non-reproducible processing conditions and lowered product quality.

Static mixers economically promote the mixing of flowing fluid streams. One typical static mixer, the helical static mixer, consists of left- and right-twisting helical elements placed at right angles to each other. The range of Reynolds numbers of practical flows for helical static mixers in industry is usually from very small values to not very large values (e.g., $\operatorname{Re}=5,000)$.

This thesis describes how static mixing processes of single-phase Newtonian and also non-Newtonian liquids can be simulated numerically and provides useful information that can be extracted from the simulation results. The Turbulent flow case is solved using the most common Reynolds Averaged Navier-Stocks (RANS) models as well as Large-Eddy 
Simulation (LES) turbulent flow model. The numerical simulation of the mixing in the helical static mixer has been performed via a two-step procedure. In the first step, the flow velocity (and the pressure) is computed. These values are then used as input to the next step. In the second step the particle trajectory in the flow field is calculated. At the entry of the pipe inlet, a large number of marker particles are uniformly distributed over half of the flow field. This represents a simplified model for diametrical feeding of the mixer with two liquids.

Using different measurement tools, such as Residence Time Distribution (RTD) and Particles Distribution Uniformity (PDU), the performance of a six-element helical static mixer is studied.

It is shown that the Reynolds number has a major impact on the performance of a static mixer.

It is also shown that the performance of a helical static mixer is different for Newtonian and non-Newtonian fluids in non-creeping flows.

Finally, heat transfer within a helical static mixer is investigated. The effects of different flow conditions on the performance of the mixer are studied. It is shown that the helical static mixer is more effective for low Reynolds number laminar flows. 


\section{ACKNOWLEDGMENTS}

This research was sponsored by Illino is Tool Works, Inc. 


\section{Table of Contents}

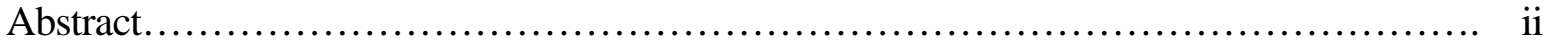

Acknowledgments.................................................................. iv

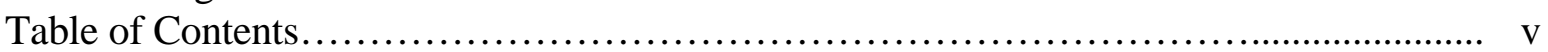

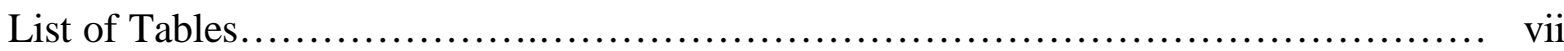

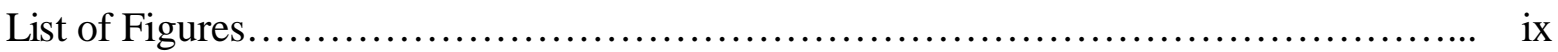

Nomenclature............................................................... xiv

Chapter 1: Introduction................................................................... 1

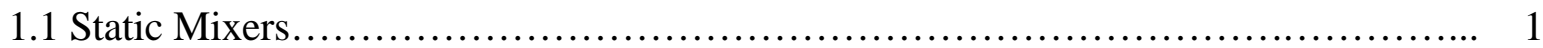

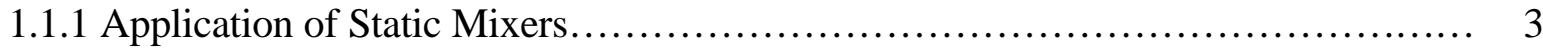

1.2 Thesis Objectives and Outlines............................................ 4

Chapter 2: Previous Studies of Static Mixers...................................... 12

2.1Experimental Studies of Static Mixers...................................... 12

2.1.1 Empirical Correlations for Static Mixer Design Parameters............................... 14

2.2 Numerical Modeling of Static Mixers................................................ 19

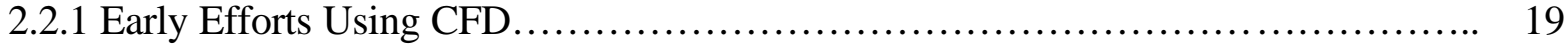

2.2.2 Recent CFD Modeling...................................................... 20

Chapter3: Analysis and Numerical Method ..................................... 30

3.1 Incompressible Flows..................................................... 30

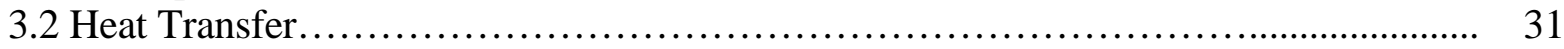

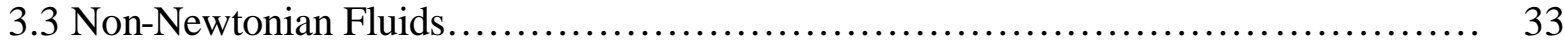

3.3.1 Time-Independent Non-Newtonian Fluids................................. 34

3.3.2 Time-Dependent Non-Newtonian Fluids................................... 39

3.3.3 Viscoelastic Fluids............................................................. 41

3.4 Non-Newtonian Fluid Modeling................................................. 44

3.5 Turbulence Modeling ...................................................... 45

3.5.1 Comments on the Physics of Turbulence...................................... 48

3.5.2 A Brief History of Turbulence Modeling........................................ 51

3.5.3 The Closure Problem.................................................. 57

3.5.4 The Reynolds Stress Model................................................. 60

3.5.5 The Large-Eddy Simulation or LES Model.................................. 60

3.6 Numerical Method........................................................ 62

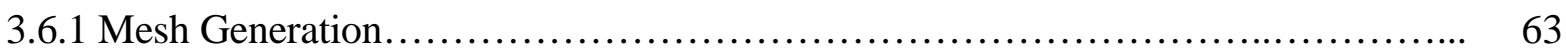

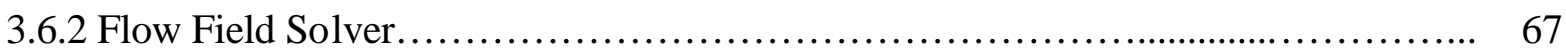

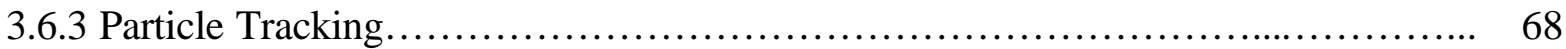

Chapter 4: Static Mixer Performance Measurement....................................... 79

4.1 Pressure Drop ............................................................ 79

4.2 Temperature Difference Ratio.............................................. 80

4.3 Heat Flux Ratio........................................................... 81

4.4 Residence Time Distribution............................................... 81

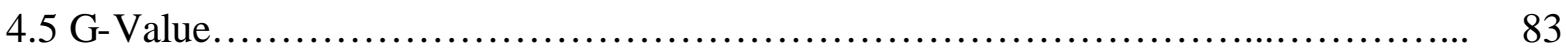

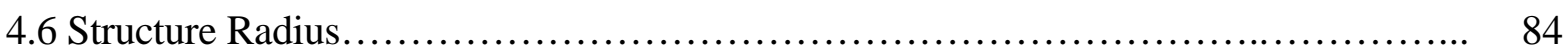

4.7 Particles Distribution Uniformity ........................................ 85

4.8 Static Mixer Efficiency.................................................... 89 
Chapter 5: Creeping and Laminar Flows in Helical Static Mixer.

5.1 Numerical Solution Accuracy................................................ 92

5.1.1 Solution Accuracy Evaluation............................................ 93

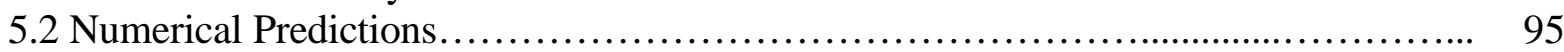

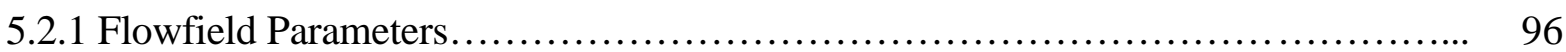

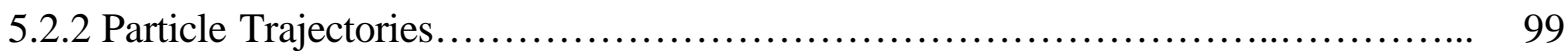

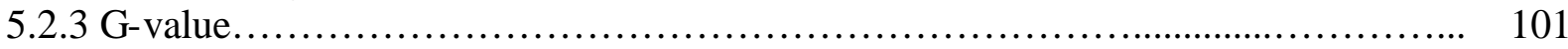

5.2.4 Residence Time Distribution............................................... 102

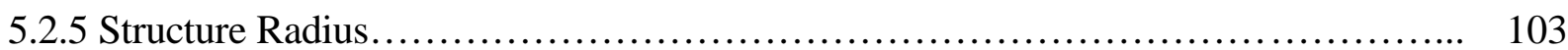

5.2.6 Particles Distribution Uniformity........................................... 104

5.2.7 Helical Static Mixer Efficiency............................................ 106

Chapter 6: Mixing of Non-Newtonian Fluids in a Helical Static Mixer................ 143

6.1: Non-Newtonian Material: Carboxymethyl Cellulose Solution in

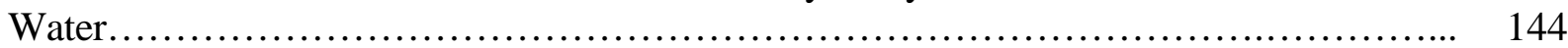

6.2 Numerical Predictions.......................................................... 147

6.2.1 Flowfield Parameters................................................... 148

6.2.2 Particle Trajectories....................................................... 151

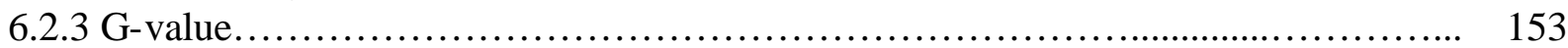

6.2.4 Residence Time Distribution............................................. 154

6.2.5 Structure Radius....................................................... 156

5.2.6 Particles Distribution Uniformity (PDU)................................... 158

6.2.7 Helical Static Mixer Efficiency............................................. 159

Chapter 7: Helical Static Mixer Performance under Turbulent Flow Regime

Conditions.......................................................................................................

7.1 RANS Turbulent Models........................................................ 218

7.1.1 Numerical Approach......................................................... 226

7.1.2 Numerical Solution Accuracy............................................. 227

7.2 Results and Discussion Using RANS Models ..................................... 229

7.3 Large-Eddy Simulation.................................................. 236

7.3.1 Numerical Approach.................................................... 239

7.4 Results and Discussion Using a LES Turbulence Model .......................... 241

Chapter 8: Heat Transfer Rate in a Helical Static Mixer............................ 282

8.1 Numerical Method........................................................... 283

8.2 Numerical Solution Accuracy............................................. 285

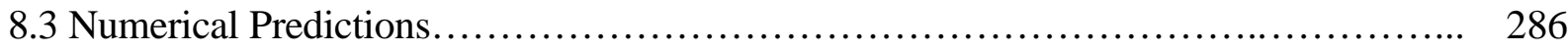

Conclusions..................................................................... 317

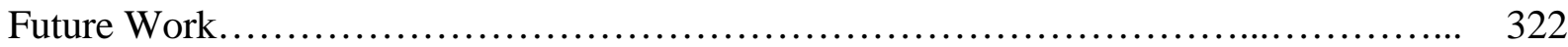

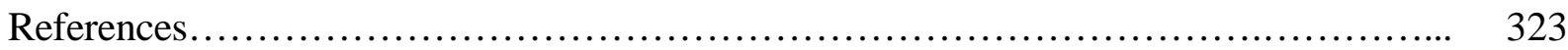




\section{LIST OF TABLES}

Number

Page

\section{Chapter 1:}

Table 1.1: Static mixer geometry 8

Table 1.2: Applications of static mixers in industries 9

Chapter 2:

Table 2.1: Summery of recent numerical studies on static mixers 28

Chapter 3:

Table 3.1: Mesh quality tests result

Table 3.2: Mesh information for the turbulent flow cases using the $k-\omega$ model 73

Chapter 5:

Table 5.1: Mean axial velocities and velocity magnitudes (m/s) 108

Table 5.2: Vorticity magnitude at each mixing element 109

Table 5.3: Pressure drop ratio for a six-element helical static mixer 110

Table 5.4: Helical Static Mixer Efficiency 111

Chapter 6:

Table 6.1: Mass flow rates for different Reynolds number and different CMC concentrations

Table 6.2: Rheological properties of CMC solutions

Table 6.3: Pressure drop prediction for different Reynolds number and different CMC concentrations

Table 6.4: Vorticity magnitude at 2nd, 4th, and 6th mixing element

(CMC concentration level of $5000 \mathrm{ppm}$ )

Table 6.7: Pressure drop comparison

165

Table 6.8: Standard deviation of the distribution function 166

Table 6.9: PDU values at the sixth mixing element 167

Table 6.10: Helical Static Mixer Efficiency 168

Chapter 7:

Table 7.1: Mesh information for the turbulent flow cases $(\operatorname{Re}=3,000)$

Table 7.2: Convergence of pressure drop across the mixer 248

Table 7.3: CPU time and memory used by different turbulence models $(\operatorname{Re}=$ $3,000)$

Table 7.4: Vorticity magnitude at $2 \mathrm{nd}, 4 \mathrm{th}$, and 6th mixing element

$(\operatorname{Re}=3,000)$

Table 7.5: Pressure drop predicted by different turbulence models

$(\operatorname{Re}=3,000)$

Table 7.6: G-value for a six-element static mixer $(\mathrm{Re}=3,000)$

Table 7.7: Structure radius at $2 \mathrm{nd}$, 4 th, and 6th mixing element

$(\operatorname{Re}=3,000)$

253

Table 7.8: Standard deviation of distribution function 254

Table 7.9: Mesh quality test results for the LES case 255

Table 7.10: Maximum, minimum, and averaged vorticity magnitudes $(\operatorname{Re}=3,000) 256$

Chapter 8: 
Table 8.1: Values of thermal conductivity and viscosity for water

Table 8.2: Averaged and minimum fluid temperatures for a pipe with static mixer (wall temperature $=318.15 \mathrm{~K}, \mathrm{BC}(\mathrm{A})$ )

Table 8.3: Averaged and minimum fluid temperatures for a pipe with no mixer (wall temperature $=318.15 \mathrm{~K}$ )

Table 8.4: Minimum to averaged temperature ratio (BC (A))

Table 8.5: Heat flux ratio (BC (A))

Table 8.6: Averaged and minimum fluid temperatures for a pipe with static mixer (wall temperature $=318.15 \mathrm{~K}, \mathrm{BC}(\mathrm{T})$ )

Table 8.7: Minimum to averaged temperature ratio $\left(\mathrm{T}_{\mathrm{W}}=318.15 \mathrm{~K}, \mathrm{BC}(\mathrm{T})\right)$

Table 8.8: Maximum to minimum temperature ratio $(\mathrm{Re}=1000, \mathrm{BC}(\mathrm{T}))$

Table 8.9: Ratio of heat flux with BC (T) to heat flux with BC (A) 303

Table 8.10: Temperature Difference Ratio $\left(\mathrm{T}_{\mathrm{W}}=348.15 \mathrm{~K}\right)$ 


\section{LIST OF FIGURES}

Number

Page

\section{Chapter 1:}

Figure 1.1: A six-element static mixer $\quad 10$

Figure 1.2: The helical static mixer studied in this research 11

\section{Chapter2:}

Figure 2.1: Progressive mixing in a helical static mixer (Kenics mixer)

\section{Chapter 3:}

Figure 3.1: Flow curves for Newtonian and time-independent non-Newtonian Fluids

Figure 3.2: Computational domain across one helical surface is cut by a large number of parallel planes perpendicular to the flowfield centerline

Figure 3.3: Cross-sectional mesh across one helical surface

Figure 3.4: Volume mesh for one mixing element

Figure 3.5: Trajectory of one particle through the mixer

\section{Chapter 4:}

Figure 4.1: A flow cross-section divided by similar strips with equal area

\section{Chapter 5:}

Figure 5.1a: The obtained structure radius for $R e=10$

Figure 5.1b: The obtained structure radius for $\mathrm{Re}=100$

Figure 5.2a: The computed pressure drop for $\mathrm{Re}=10$

Figure 5.2b: The computed pressure drop for $\operatorname{Re}=100$

Figure 5.3: Images of particle distribution at $2^{\text {nd }}$ element for $\mathrm{Re}=1000$

Figure 5.4: Residual curves, from top to bottom: $\operatorname{Re}=0.01,10$, and 1000

Figure 5.5: Velocity field, from top to bottom: at 2nd, 4th, and 6th mixing elements $(\operatorname{Re}=1)$

Figure 5.6: Velocity field, from top to bottom: 2nd, 4th, and 6th mixing elements $(\operatorname{Re}=10)$

Figure 5.7: Velocity field, from top to bottom: at 2nd, 4th, and 6th mixing elements $(\operatorname{Re}=100)$

Figure 5.8: Velocity field, from top to bottom: at 2nd, 4th, and 6th mixing elements $(\mathrm{Re}=1000)$

Figure 5.9: Vorticity contours (1/s), from left to right, top to bottom: at 1st, 2nd, 3rd, 4th, 5th, and 6th mixing elements $(\operatorname{Re}=1)$

Figure 5.10: Vorticity contours (1/s), from left to right, top to bottom: at 1st, 2nd, 3rd, 4th, 5th, and 6th mixing elements $(\operatorname{Re}=10)$

Figure 5.11: Vorticity contours (1/s), from left to right, top to bottom: at 1st, 2nd, 3rd, 4th, 5th, and 6th mixing elements $(\operatorname{Re}=100)$

Figure 5.12: Vorticity contours (1/s), from left to right, top to bottom: at 1st, 2 nd, 3 rd, 4th, 5th, and 6th mixing elements $(\operatorname{Re}=1000)$

Figure 5.13: Particles locations at each mixing element $(\operatorname{Re}=1)$

Figure 5.14: Particles locations at each mixing element $(\operatorname{Re}=100)$

Figure 5.15: Particles locations at 2nd, 4th, and 6th elements $(\operatorname{Re}=0.01)$ 
Figure 5.16: Particles locations at 2nd, 4th, and 6th elements $(\operatorname{Re}=0.1) \quad 129$

Figure 5.17: Particles locations at 2nd, 4th, and 6th elements $(\operatorname{Re}=10)$

Figure 5.18: Particles locations at 2nd, 4th, and 6th elements $(\operatorname{Re}=1000)$

Figure 5.19: G-value (1/s) 132

Figure 5.20: Distribution function in a six-element mixer, $d t^{*}=0.01$ $(\operatorname{Re}=1)$

Figure 5.21: Distribution function in a six-element mixer, $d t^{*}=0.01$ $(\operatorname{Re}=10)$

Figure 5.22: Distribution function in a six-element mixer, $d t^{*}=0.01$ $(\operatorname{Re}=100)$

Figure 5.23: Distribution function in a six-element mixer, $d t^{*}=0.01$

$(\operatorname{Re}=1000)$

Figure 5.24: Structure radii at the end of each mixing element $(\operatorname{Re}=1) \quad 137$

Figure 5.25: Structure radii at the end of the last mixing element 138

Figure 5.26: PDU values at the end of each mixing element $(\operatorname{Re}=1) \quad 139$

Figure 5.27: PDU values at the end of each mixing element $(\operatorname{Re}=100)$

Figure 5.28: PDU values at the end of even numbered mixing elements 141

Figure 5.29: PDU values at the fourth mixing element 142

Chapter 6:

Figure 6.1: Non-dimensionalized difference between the viscosities predicted by the power law and the Carreau models

Figure 6.2: Pressure drop across a six-element helical static mixer for a CMC concentration level of $50 \mathrm{ppm}$

Figure 6.3: Pressure drop across a six-element helical static mixer for a CMC concentration level of $500 \mathrm{ppm}$

Figure 6.4: Pressure drop across a six-element helical static mixer for a CMC concentration level of $5000 \mathrm{ppm}$

Figure 6.5: Velocity field at 2nd, 4th, and 6th mixing element $\left(\operatorname{Re}^{\prime}=1\right)$

Figure 6.6: Velocity field at 2nd, 4th, and 6th mixing element $\left(\operatorname{Re}^{\prime}=10\right)$

Figure 6.7: Velocity field at 2nd, 4th, and 6th mixing element $\left(\operatorname{Re}^{\prime}=100\right)$

Figure 6.8: Velocity field at 2nd, 4th, and 6th mixing element $\left(\operatorname{Re}^{\prime}=1000\right)$

Figure 6.9: Particles locations at 2nd, 4th, and 6th mixing element for a CMC concentration level of $50 \mathrm{ppm}\left(\operatorname{Re}^{\prime}=0.1\right)$

Figure 6.10: Particles locations at $2 \mathrm{nd}$, 4th, and 6th mixing element for a CMC concentration level of $50 \mathrm{ppm}\left(\mathrm{Re}^{\prime}=1\right)$

Figure 6.11: Particles locations at 2nd, 4th, and 6th mixing element for a CMC concentration level of $50 \mathrm{ppm}\left(\mathrm{Re}^{\prime}=10\right)$

Figure 6.12: Particles locations at 2nd, 4th, and 6th mixing element for a CMC concentration level of $50 \mathrm{ppm}\left(\mathrm{Re}^{\prime}=100\right)$

Figure 6.13: Particles locations at $2 \mathrm{nd}$, 4th, and 6th mixing element for a CMC concentration level of $50 \mathrm{ppm}\left(\mathrm{Re}^{\prime}=1000\right)$

Figure 6.14: Particles locations at 2nd, 4th, and 6th mixing element for a CMC concentration level of $500 \mathrm{ppm}\left(\mathrm{Re}^{\prime}=0.1\right)$

Figure 6.15: Particles locations at 2nd, 4th, and 6th mixing element for a CMC concentration level of $500 \mathrm{ppm}\left(\mathrm{Re}^{\prime}=1\right)$ 
Figure 6.16: Particles locations at 2nd, 4th, and 6th mixing element for a CMC concentration level of $500 \mathrm{ppm}\left(\mathrm{Re}^{\prime}=10\right)$

Figure 6.17: Particles locations at 2nd, 4th, and 6th mixing element for a CMC concentration level of $500 \mathrm{ppm}\left(\mathrm{Re}^{\prime}=100\right)$

Figure 6.18: Particles locations at 2nd, 4th, and 6th mixing element for a CMC concentration level of $500 \mathrm{ppm}\left(\mathrm{Re}^{\prime}=1000\right)$

Figure 6.19: Particles locations at 2nd, 4th, and 6th mixing element for a CMC concentration level of $5000 \mathrm{ppm}\left(\mathrm{Re}^{\prime}=0.1\right)$

Figure 6.20: Particles locations at 2nd, 4th, and 6th mixing element for a CMC concentration level of $5000 \mathrm{ppm}\left(\mathrm{Re}^{\prime}=1\right)$

Figure 6.21: Particles locations at 2nd, 4th, and 6th mixing element for a CMC concentration level of $5000 \mathrm{ppm}\left(\mathrm{Re}^{\prime}=10\right)$

Figure 6.22: Particles locations at 2nd, 4th, and 6th mixing element for a CMC concentration level of $5000 \mathrm{ppm}\left(\mathrm{Re}^{\prime}=100\right)$

Figure 6.23: Particles locations at 2nd, 4th, and 6th mixing element for a CMC concentration level of 5000 ppm $\left(\operatorname{Re}^{\prime}=1000\right)$

Figure 6.24: Gvalues for a six-element mixer (1/s) CMC concentration level: 50 ppm

Figure 6.25: G-values for a six-element mixer (1/s) CMC concentration level: 50 ppm

Figure 6.26: G-values for a six-element mixer (1/s) CMC concentration level: 50 ppm

Figure 6.27: Distribution function for CMC solutions flow in the six-element static mixer $\left(\operatorname{Re}^{\prime}=0.1\right.$, Concentration level: $50 \mathrm{ppm}, d t^{*}=0.01$ )

Figure 6.28: Distribution function for CMC solutions flow in the six-element static mixer $\left(\mathrm{Re}^{\prime}=0.1\right.$, Concentration level: $500 \mathrm{ppm}, d t^{*}=0.01$ )

Figure 6.29: Distribution function for CMC solutions flow in the six-element static mixer $\left(\operatorname{Re}^{\prime}=0.1\right.$, Concentration level: $5000 \mathrm{ppm}, d t^{*}=0.01$ )

Figure 6.30: Distribution function for CMC solutions flow in the six-element static mixer ( $\operatorname{Re}^{\prime}=1$, Concentration level: $50 \mathrm{ppm}, d t^{*}=0.01$ )

Figure 6.31: Distribution function for CMC solutions flow in the six-element static mixer $\left(\operatorname{Re}^{\prime}=1\right.$, Concentration level: $500 \mathrm{ppm}, d t^{*}=0.01$ )

Figure 6.32: Distribution function for CMC solutions flow in the six-element static mixer ( $\mathrm{Re}^{\prime}=1$, Concentration level: $5000 \mathrm{ppm}, d t^{*}=0.01$ )

Figure 6.33: Distribution function for CMC solutions flow in the six-element static mixer ( $\operatorname{Re}^{\prime}=10$, Concentration level: $50 \mathrm{ppm}, d t^{*}=0.01$ )

Figure 6.34: Distribution function for CMC solutions flow in the six-element static mixer ( $\mathrm{Re}^{\prime}=10$, Concentration level: $500 \mathrm{ppm}, d t^{*}=0.01$ )

Figure 6.35: Distribution function for CMC solutions flow in the six-element static mixer ( $\mathrm{Re}^{\prime}=10$, Concentration level: $5000 \mathrm{ppm}, d t^{*}=0.01$ )

Figure 6.36: Distribution function for CMC solutions flow in the six-element static mixer $\left(\mathrm{Re}^{\prime}=100\right.$, Concentration level: $50 \mathrm{ppm}, d t^{*}=0.01$ )

Figure 6.37: Distribution function for CMC solutions flow in the six-element static mixer $\left(\operatorname{Re}^{\prime}=100\right.$, Concentration level: $500 \mathrm{ppm}, d t^{*}=0.01$ ) 
Figure 6.38: Distribution function for CMC solutions flow in the six-element static mixer $\left(\mathrm{Re}^{\prime}=100\right.$, Concentration level: $\left.5000 \mathrm{ppm}, d t^{*}=0.01\right)$

Figure 6.39: Distribution function for CMC solutions flow in the six-element static mixer ( $\operatorname{Re}^{\prime}=1000$, Concentration level: $\left.50 \mathrm{ppm}, d t^{*}=0.01\right)$

Figure 6.40: Distribution function for CMC solutions flow in the six-element static mixer $\left(\mathrm{Re}^{\prime}=1000\right.$, Concentration level: $500 \mathrm{ppm}, d t^{*}=0.01$ )

Figure 6.41: Distribution function for CMC solutions flow in the six-element static mixer ( $\mathrm{Re}^{\prime}=1000$, Concentration level: $5000 \mathrm{ppm}, d t^{*}=0.01$ )

Figure 6.42: Structure radii for $\operatorname{Re}^{\prime}=1$ (CMC concentration level: 5000 ppm) 210

Figure 6.43: Structure radii at the end of the last mixing element (CMC concentration level: $5000 \mathrm{ppm}$ )

Figure 6.44: PDU values at the end of even numbered mixing elements (CMC concentration level: 50ppm)

Figure 6.45: PDU values at the end of even numbered mixing elements (CMC concentration level: $500 \mathrm{ppm}$ )

Figure 6.46: PDU values at the end of even numbered mixing elements (CMC concentration level: 5000ppm)

Figure 6.47: Helical static mixer efficiency

\section{Chapter 7:}

Figure 7.1: Velocity contours $(\mathrm{m} / \mathrm{s})$ and the vorticity contours $(1 / \mathrm{s})$ for $\operatorname{Re}=5,000$ (using the $k$-? model)

Figure 7.2: Velocity field, from top to bottom: at 2nd, 4th and 6th mixing element, predicted by Spalart-Allmaras model

Figure 7.3: Velocity field, from top to bottom: at 2nd, 4th, and 6th mixing element, predicted by standard $k$-e model

Figure 7.4: Velocity field, from top to bottom: at 2nd, 4th, and 6th mixing element, predicted by SST $k-$ ? model

Figure 7.5: Velocity field, from top to bottom: at 2nd, 4th, and 6th mixing element, predicted by RSM model

Figure 7.6: Velocity contours $(\mathrm{m} / \mathrm{s})$, from top to bottom: at $2 \mathrm{nd}$, 4th, and 6th element, predicted by Spalart-Allmaras model

Figure 7.7: Velocity contours $(\mathrm{m} / \mathrm{s})$, from top to bottom: at $2 \mathrm{nd}$, 4th, and 6th element, predicted by standard $k$-e model

Figure 7.8: Velocity contours $(\mathrm{m} / \mathrm{s})$, from top to bottom: at $2 \mathrm{nd}$, 4 th, and 6 th element, predicted by standard SST $k-$ ? model

Figure 7.9: Velocity contours (m/s), from top to bottom: at $2 \mathrm{nd}$, 4th, and 6 th element, predicted by standard RSM model

Figure 7.10: Particles locations at 2nd, 4th, and 6th element

Predicted by Spalart-Allmaras model

Figure 7.11: Particles locations at 2nd, 4th, and 6th element

Predicted by standard $k$-e model

Figure 7.12: Particles locations at 2nd, 4th, and 6th element

Predicted by SST $k-$ ? model

Figure 7.13: Particles locations at 2nd, 4th, and 6th element 
Figure 7.14: Distribution function for flow in the six-element static mixer, predicted by Spalart-Allmaras model

Figure 7.15: Distribution function for flow in the six-element static mixer, predicted by standard $k-e$ model

Figure 7.16: Distribution function for flow in the six-element static mixer, predicted by SST $k-$ ? model

Figure 7.17: Distribution function for flow in the six-element static mixer, predicted by RSM model

Figure 7.18: Static pressure at the upstream and the down stream locations of a six-element static mixer $(\mathrm{Pa})$

Figure 7.19: Static pressure at end of the 1st, 2nd, 3rd, 4th, 5th, and 6th mixing elements $(\mathrm{Pa})$

Figure 7.20: Cross-sectional velocity vectors at each mixing ele ment cross section (from left to right, top to bottom at the end of the 1st, 2nd, 3rd, 4th, 5th, and 6th elements)

Figure 7.21: Particle locations the end of each mixing element (from left to right, top to bottom at the end of 1st, 2nd, 3rd, 4th, 5th, and 6th elements)

Figure 7.22: Particle locations the end of the 2nd, 4th, and 6th mixing elements, respectively from left to right (Left: at physical time $=0.7509 \mathrm{~s}$, Right: at physical time $=0.80546 \mathrm{~s}$ )

Figure 7.23: Distribution function for flow in the six-element helical static mixer

Figure 7.24: PDU values at the six mixing elements

Figure 7.25: Static pressure at the upstream and the down stream of a six-element static mixer (Pa), predicted by $k-$ ? (top) and RSM (bottom) models

\section{Chapter 8:}

Figure 8.1: Comparison between numerical and experimental data 305

Figure 8.2: Temperature at even numbered elements, $\operatorname{Re}=10 \quad 306$

Figure 8.3: Temperature at even numbered elements, $\mathrm{Re}=100$

Figure 8.4: Temperature at even numbered elements, $\operatorname{Re}=1000 \quad 308$

Figure 8.5: Temperature contours at the same cross-sections, $\operatorname{Re}=10$

Figure 8.6: Temperature contours at the same cross-sections, $\operatorname{Re}=100 \quad 310$

Figure 8.7: Temperature contours at the same cross-sections, $\operatorname{Re}=1000$

Figure 8.8: Heat transfer coefficient in a pipe $(\operatorname{Re}=1, \mathrm{BC}(\mathrm{A}))$

Figure 8.9: Heat transfer coefficient in a pipe $(\operatorname{Re}=100, \mathrm{BC}(\mathrm{A}))$

Figure 8.10: Heat transfer coefficient in a pipe $(\operatorname{Re}=1000, B C(A))$

Figure 8.11: Temperature contours, $\mathrm{BC}(\mathrm{T}), \mathrm{Re}=100$

Figure 8.12: Temperature contours, BC (T), $\mathrm{Re}=1000$ 


\section{Nomenclature}

\begin{tabular}{|c|c|}
\hline$c_{p}$ & specific heat \\
\hline$d$ & pipe diameter \\
\hline$E$ & total energy \\
\hline$F_{i}$ & external force vector $(i=1,2,3)$ \\
\hline$g$ & acceleration due to gravity \\
\hline$H(x)$ & the Heaviside function ( $=x$ if $x>0,=0$ otherwise) \\
\hline$h$ & enthalpy \\
\hline$K$ & thermal conductivity \\
\hline$k$ & turbulence kinetic energy \\
\hline$L$ & mixing element length \\
\hline$\dot{m}$ & mass flow rate \\
\hline$N_{p}$ & particles released at the flowfield inlet \\
\hline$n$ & power law index \\
\hline$P D U$ & particles distribution uniformity \\
\hline$p$ & pressure \\
\hline$p d_{1}(i)$ & particle distribution function of the first kind $\left(i=1, \ldots, N_{s}\right)$ \\
\hline$p d_{2}(i)$ & particle distribution function of the second kind $\left(i=1, \ldots, N_{l}\right)$ \\
\hline$Q$ & volumetric flow rate \\
\hline $\operatorname{Re}$ & Reynolds number $(=\rho U d / \mu)$ \\
\hline $\operatorname{Re}^{\prime}$ & modified Reynolds number of non-Newtonian fluid \\
\hline$r_{s}$ & structure radius \\
\hline $\bar{S}_{i j}$ & rate of strain tensor \\
\hline$\Delta s$ & computational grid interval size \\
\hline$T$ & temperature \\
\hline$T_{0}$ & reference temperature \\
\hline$T_{W}$ & wall temperature \\
\hline$t^{*}$ & non-dimensionalized residence time \\
\hline$U$ & bulk velocity \\
\hline$u_{i}$ & velocity vector $(i=1,2,3)$ \\
\hline$V$ & grid cell volume \\
\hline$V_{m}$ & mixer volume \\
\hline$x_{i}$ & position vector $(i=1,2,3)$ \\
\hline$\dot{\gamma}$ & shear rate \\
\hline$\delta_{i j}$ & Kroncker delta \\
\hline$\varepsilon$ & turbulence energy dissipation \\
\hline$\kappa$ & consistency index \\
\hline$\lambda$ & time constant in the Carreau law \\
\hline$\mu$ & molecular viscosity \\
\hline$\rho$ & density \\
\hline
\end{tabular}


- $\rho \overline{u_{i}^{\prime} u_{j}^{\prime}} \quad$ Reynolds stresses $(i, j=1,2,3)$

$\tau_{i j} \quad$ stress tensor $(i, j=1,2,3)$

$\tau_{w} \quad$ wall shear stress

$\omega \quad$ specific dissipation 


\section{The University of Toledo}

\section{College of Engineering}

\section{HEREBY RECOMMEND THAT THE DISSERTATION PREPARED UNDER MY}

SUPERVISION BY Ramin Khosravi Rahmani

ENTITLED

THREE-DIMENSIONAL NUMERICAL SIMULATION AND PERFORMANCE STUDY OF AN INDUSTRIAL HELICAL STATIC MIXER

BE ACCEPTED IN PARTIAL FULFILLMENT OF THE REQUIREMENTS FOR

THE DEGREE OF DOCTOR OF PHILOSOPHY IN ENGINEERING

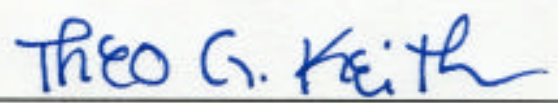

Dissertation Advisor: Professor Theo G. Keith

Recommendation concurred by

\section{Ardollen affh}

Committee member 2: Professor A. Afjeh

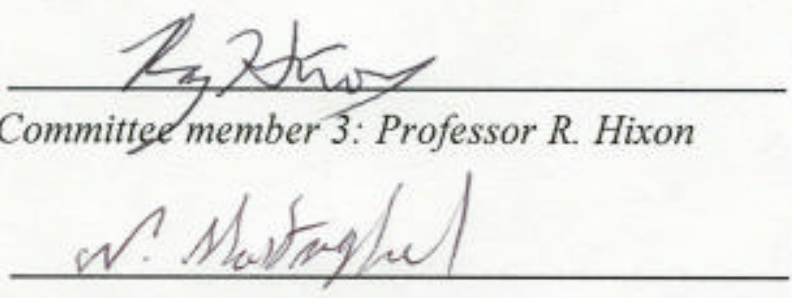

Committee

Committee member 4: Professor N. Mostaghel

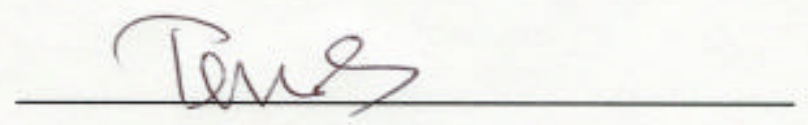

Committee member 5: Professor T. $\mathrm{Ng}$

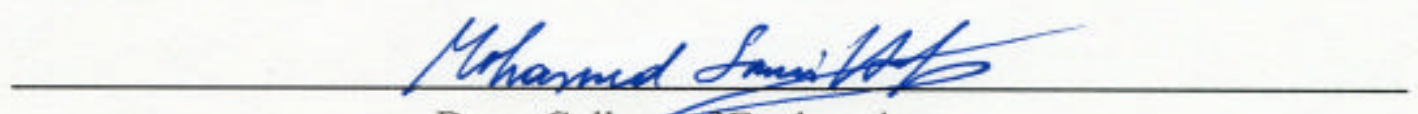

Dean, College of Engineering 


\section{CHAPTER ONE}

\section{INTRODUCTION}

\subsection{Static Mixers}

Viscous liquids have to be homogenized in continuous operations in many branches of processing industries. Consequently, fluid mixing plays a critical role in the success or failure of these processes. Mixing is an essential component of nearly all industrial chemical processes, ranging from simple blending to complex multi-phase reaction systems for which the reaction rate, the yield and the selectivity are highly dependent upon the mixing performance. Consequences of improper mixing include non-reproducible processing conditions and lowered product quality, resulting in the need for more elaborate downstream purification processes and increased waste disposal costs.

Static mixers have been utilized over a wide range of applications such as continuous mixing, blending, heat and mass transfer processes, chemical reactions, etc. The static mixer has increased in popularity within the chemical industry over recent years. The mixing elements, which are called segments, as one could imagine, appear to have been cut from a long periodic structure. Several of these segments are inserted in-line at various locations, and housed in a pipe which squeezes the liquid through the resulting mixing 
element. Despite wide spread usage, the way these mixers work is still not fully understood.

In recent years, significant progress has been made in the characterization of fluidmechanical mixing using Lagrangian tracking techniques and tools from dynamical systems theory, particularly those related to chaos. The bulk of the relevant previous work has focused on model flows that are two-dimensional and time periodic, and a smaller set of studies have considered simple, three-dimensional, spatially periodic flows $[1,2]$ where a simplified, two-dimensional analytical approximation to the velocity field was obtained. While complex, three-dimensional geometries typical of most industrial mixers make analytic solutions of the velocity field in such equipment impractical; a high quality numerical solution of the velocity field can provide a suitable starting point to characterize mixing performance. This approach has allowed Lagrangian techniques to be applied to a fully three-dimensional flow in an industrial helical static mixer. The helical static mixer consists of left- and right-twisting helical elements at right angles to each other. Each element twists through an angle of $180^{\circ}$. The complete mixer consists of a series of elements with alternating clockwise and counter-clockwise twist arranged axially within a pipe so that the leading edge of an element is at right angles to the trailing edge of the previous element. Figure 1.1 shows an illustration of a six-element helical static mixer. The bulk flow direction and leading edge and trailing edge of the second mixing element are shown in this figure.

The static mixer studied here is an industrial helical static mixer: the TAH static mixer (Robbinsville, NJ). The mixer geometrical parameters are shown in Table 1.1. The solid material thickness of the mixer is about $22 \%$ of each segment length, which makes the zero 
thickness assumption of the mixer, as has been used in previous studies, questionable. Also, the ratio of the each segment length to the mixer diameter is about 0.846 . In a standard Kenics mixer, the segment length is 1.5-2.5 times the mixer diameter. Figure 1.2 shows a picture of the mixer and compares its size to tip of a pen.

Mixing in a helical static mixer occurs because the elements:

Divide and re-combine the fluid at the leading and trailing edge of each element; Produce a strong flow perpendicular to the pipe centerline; Increase the energy dissipation and therefore increase the energy available for mixing compared to an equivalent length of pipe that does not contain mixing elements.

Pahl and Muschelknautz [3] list thirty different static mixers that were commercially available in 1979. Many of them are still available. The ones that have been studied the most are the helical mixer and Koch-Sulzer mixers e.g., SMV, SMX, and SMXL.

Some of the advantages of static mixers compared to other kinds of mixers are: No moving parts (therefore, low maintenance), No external energy required, and Few clogging problems compared to some other mixers.

\subsubsection{Application of Static Mixers}

While mechanical agitators are commonly used for batch mixing, static mixers are often preferred for continuous mixing applications. Helical mixers are used in a wide variety of applications. For example, they are used in a reactor to produce polymers and in a turbulent flow vessel to make liquid-liquid dispersions. In contrast, Koch-Sulzer mixers 
tend to be used for specific applications over a restricted range of flow rates. As an example, the SMV mixer is used for gas-liquid contacting and SMF is used for viscous flows where clogging is a concern.

Helical static mixers are used for process applications, such as blending for $\mathrm{pH}$ control, polymer dilution, and gas-liquid or solid-liquid contacting. Helical static mixers have been used in wastewater treatment. They are used for high and low pressure spray applications. They are also used in order to increase the rate of heat transfer in heat exchangers. The radial mixing action rapidly eliminates temperature gradients, reducing fouling and thermal degradation. The main applications of static mixers are categorized and shown in Table 1.2.

\subsection{Thesis Objectives and Outlines}

The objectives of this research are:

to develop and validate a 3D CFD model of laminar and turbulent flows in helical static mixers;

to apply this model to elucidate the characteristic s of the flow in helical static mixers over a wide range of Reynolds numbers;

to study the heat transfer rate in these mixers made of either heat conductive or heat resistance materials;

to study the performance of these mixers for Newtonian and non-Newtonian working fluids; 
to demonstrate the benefits of helical static mixers from understanding the flow physics; and

to use the model to identify critical research issues as a guide to future research in this area.

This dissertation is organized as follows:

Chapter 2 reviews previous experimental and numerical studies of static mixers. The majority of the experimental studies of mixers are concentrated on design parameters for these mixers. These parameters may be immediately used in the design process. However, the existing experimental studies of static mixers provide very little information about how the mixers work and whether their performance is high or not. Unfortunately, the lack of detailed experimental data makes the validation of a numerical model of helical static mixers a challenging task.

Several recent modeling studies of laminar flow of Newtonian fluids in helical static mixers have focused on the mixing process in these tools to the near exclusion of information regarding the velocity field. In contrast, very few modeling studies of the flow of non-Newtonian fluids in static mixers have been published. Moreover, there is only one published paper on the numerical simulation of turbulent flow in helical static mixer. That work employed a $k-\omega$ turbulence model. To the best knowledge of the author, there is no published numerical study of heat transfer in a helical static mixer. Hence, a primary objective of this thesis is to provide numerical prediction of non-Newtonian fluid flow, turbulent flow, and heat transfer in helical static mixers.

Chapter 3 provides the physics needed to study the flow in a mixer and describes the numerical method used to study the performance of a helical static mixer. This chapter 
briefly explains main aspects of non-Newtonian fluids and presents two commonly used models for non-Newtonian fluids. This chapter also presents a description of the Reynolds Averaged Navier-Stokes equations (RANS) turbulence models, and also provides information about the Large-Eddy Simulation approach used in this study. Moreover, heat transfer modeling is discussed in this chapter.

Chapter 4 provides a detailed definition of parameters that are used to study the performance of a static mixer. Some of these parameters can be measured experimentally or they are determined using results of a numerical simulation. Some other parameters are based on numerical data.

Chapter 5 presents a validation of the numerical method used in this research and presents results and discussion related to helical static mixer performance for laminar flow conditions.

Chapter 6 presents the mixing of non-Newtonian fluids in a helical static mixer and discusses the mixer performance using non-Newtonian fluids and provides a comparison of mixer performance for both Newtonian and non-Newtonian fluids.

Chapter 7 deals with the turbulent flow regime in static mixers. The accuracy, global performance and costs of the different RANS turbulent models are examined. A large-eddy turbulent flow simulation of a static mixer is provided in this chapter; and the static mixer performance predicted by Large-Eddy Simulation (LES) is presented.

Chapter 8 investigates heat transfer mechanisms within a helical static mixer. The solid material of the mixer is considered to be made of both heat conductive and heat resistance materials. A comparison of the static mixer performance for both materials is provided. 
The work presented in this thesis has been presented to various, peer-reviewed, technical conferences. The majority of the work has been submitted to peer-reviewed journals for publication. 


\begin{tabular}{|l|l|}
\hline Diameter (D) & $4.80 \mathrm{~mm}$ \\
\hline Element length (L) & $4.06 \mathrm{~mm}$ \\
\hline Thickness & $0.89 \mathrm{~mm}$ \\
\hline Entrance length & $9.60 \mathrm{~mm}$ \\
\hline Exit length & $9.60 \mathrm{~mm}$ \\
\hline
\end{tabular}

Table 1.1: Static mixer geometry 


\begin{tabular}{|l|l|}
\hline \multicolumn{1}{|c|}{ Industry } & \multicolumn{1}{c|}{ Applications } \\
\hline Chemicals & $\begin{array}{l}\text { Chlorination and oxidation } \\
\text { Steam injection } \\
\text { Acid and base dilution } \\
\text { Fast reactions }\end{array}$ \\
\hline Food processing & $\begin{array}{l}\text { Acid washing of fats and oils } \\
\text { Constituent blending } \\
\text { Starch slurry cooking }\end{array}$ \\
\hline Mineral processing & $\begin{array}{l}\text { Slurry dilution } \\
\text { Metal recovery by solvent extraction }\end{array}$ \\
\hline Paints and resins & $\begin{array}{l}\text { Coloring and tinting } \\
\text { Solvent blending } \\
\text { Color concentrate dilution }\end{array}$ \\
\hline $\begin{array}{l}\text { Petrochemicals and } \\
\text { refining }\end{array}$ & $\begin{array}{l}\text { Gaseous reactant blending } \\
\text { Gasoline blending } \\
\text { Emissions monitoring and control }\end{array}$ \\
\hline Pharmaceuticals & $\begin{array}{l}\text { Nutrient blending } \\
\text { Sterilization } \\
\text { pH control }\end{array}$ \\
\hline Polymers and plastics & $\begin{array}{l}\text { Reactant-catalyst blending } \\
\text { Thermal homogenization } \\
\text { Plug-flow finishing reactors }\end{array}$ \\
\hline Pulp and paper & $\begin{array}{l}\text { Chemical and coatings preparation } \\
\text { Stock dilution and consistency control } \\
\text { Addition of bleaching chemicals }\end{array}$ \\
\hline $\begin{array}{l}\text { Water and waste } \\
\text { treatment }\end{array}$ & $\begin{array}{l}\text { Rapid mixing and polymer dilution } \\
\text { Disinfection and dechlorination } \\
\text { Aeration }\end{array}$ \\
\hline
\end{tabular}

Table 1.2: Applications of static mixers in industries 


\section{Bulk flow direction}

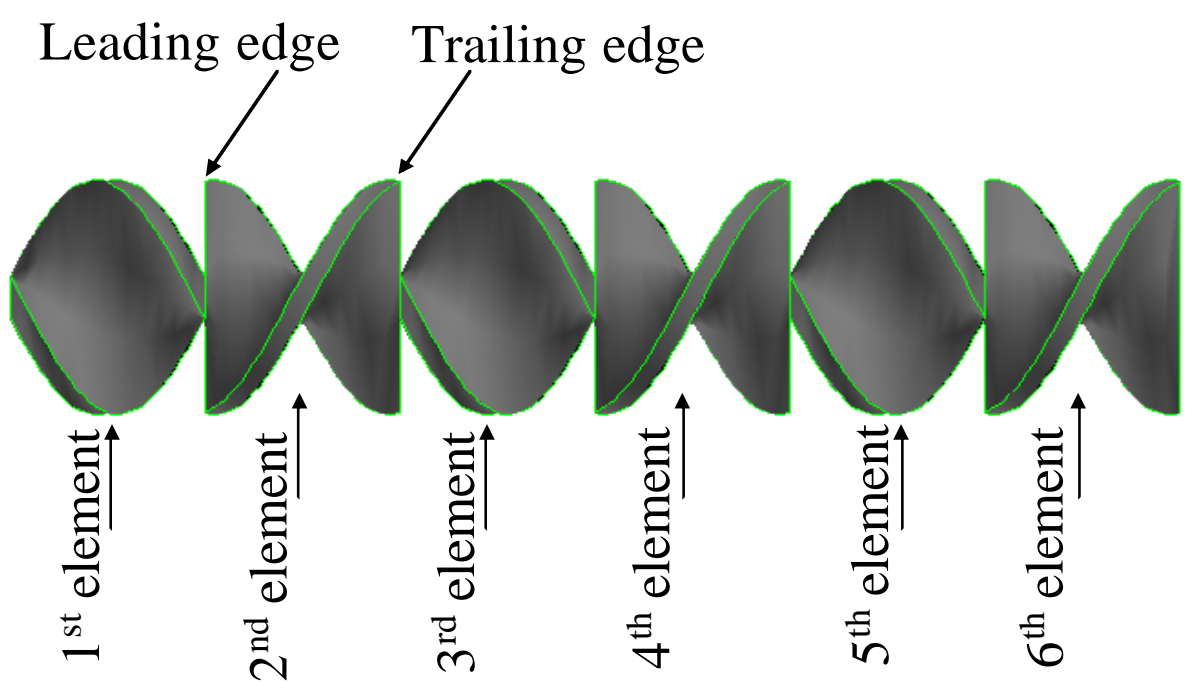

Figure 1.1: A six-element static mixer 


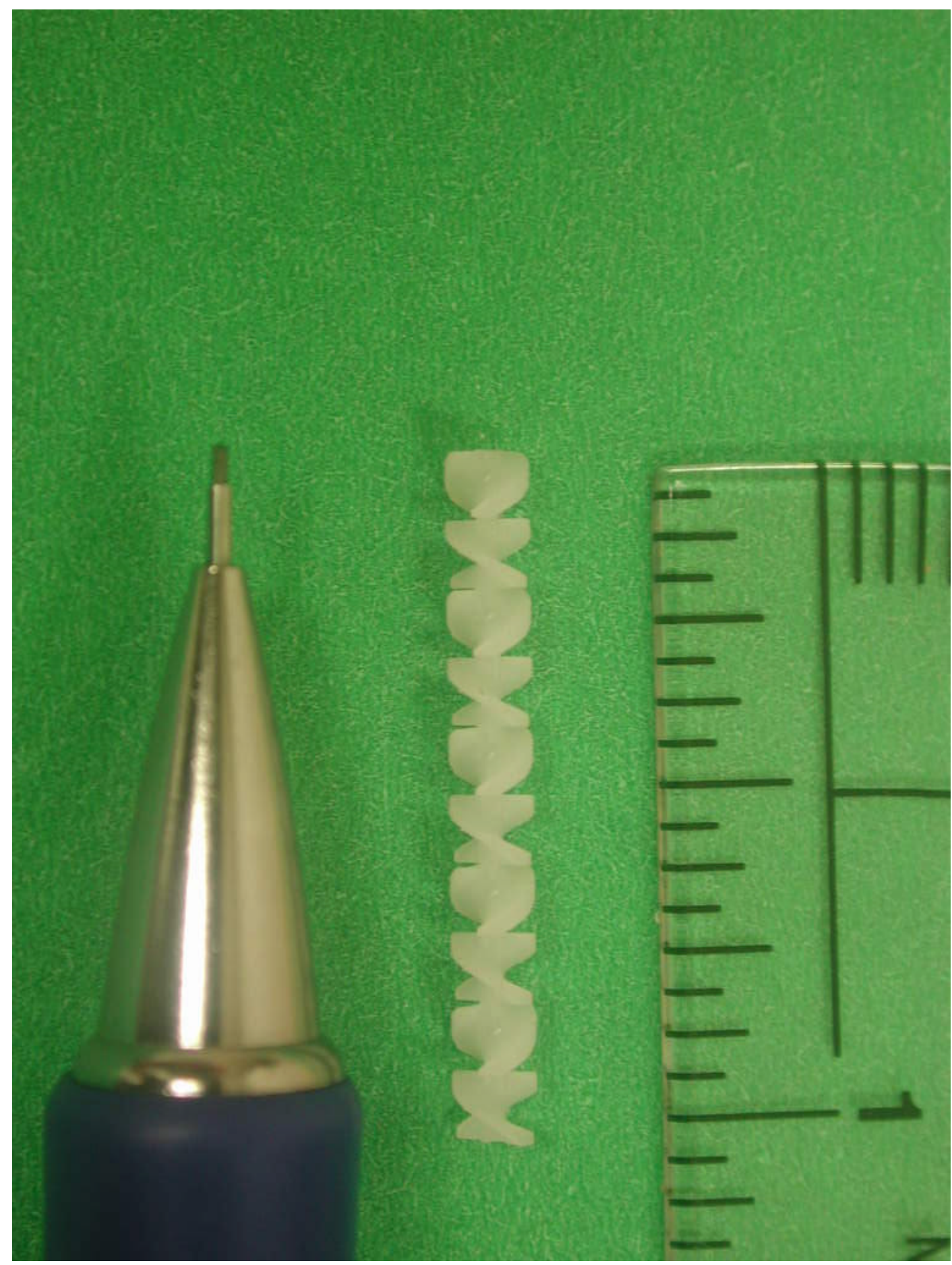

Figure 1.2: The helical static mixer studied in this research 


\section{CHAPTER TWO}

\section{PREVIOUS STUDIES OF STATIC MIXERS}

Due to the industrial importance of static mixers, many studies have been undertaken in attempts to characterize their performance. This chapter provides a two-part review of previous investigations static mixers. The first part reviews experimental studies of mixers over the past 30 years. The second part provides information about the numerical studies in this area. This part is further divided into two sub-sections: the first part is about early efforts to numerically model the flow in static mixers and the second part reviews recent numerical studies of static mixers using CFD.

\subsection{Experimental Studies of Static Mixers}

Many experimental studies have been undertaken in an attempt to characterize the performance of these devices in both laminar and turbulent flow regimes. In order to summarize the available literature, static mixer characterization studies are divided into three groups. 
The first group consists primarily of experimental measurements of basic process design parameters and the development of empirical correlations between these parameters and non-dimensional groups. This type of studies have measured and correlated basic process parameters such as pressure drop across the mixer vs. flow Reynolds number [3-7], mass transfer vs. Reynolds number and Schmidt number [8,9], heat transfer vs. Reynolds number and Prandtl numbers $[8,10,11]$, and drop size distributions produced in turbulent flow vs. Reynolds number and Weber number [12-14]. This group of experimental studies helps to provide the minimum set of parameters needed for process design using static mixers; and represents many of the earliest studies performed on static mixers. The parameters studied in these experiments are global, because they are measured before and after the mixer. The static mixer is treated as a black box and no understanding of how the static mixer affects these parameters is presented.

The goal of the work within the second group is to measure mixing performance more directly. Examples of this group include studies which have characterized mixing performance via Residence Time Distributions [15-20], mixing indices [3,21], and striation formation $[4,5,10,11,22,23]$. Characterization of static mixers is based either on the results obtained by experiment, or on an over-simplified model of the mixer behavior, without detailed measurement or calculation of the physical features of the flow. This sort of characterization represents a significant step forward toward understanding the actual performance of static mixers.

The third, and the smallest, group of experimental studies attempts to obtain fundamental information of the flow across a static mixer and the mixing process within 
and downstream of this device by using either analyt ical or numerical techniques to obtain detailed solutions for the internal velocity field [24-31]. Mixing is then evaluated via simulations, which may be compared with results from the first two groups of studies described above. This group of studies on static mixers mixer represents the forefront of current progress in mixer characterization and provides both qualitative and quantitative evaluation of performance of static mixers.

\subsubsection{Empirical Correlations for Static Mixer Design Parameters}

This section contains a review of empirical correlations that can provide basics in order to design a static mixer.

Pressure Drop: The pressure drop across a static mixer has been studied experimentally for Newtonian and non-Newtonian fluids [3,5-7,32], since it is essential in order to correctly size the extruder or pump, feeding the static mixer. A set of consistency and power law indices for the flow of a CMC solution inside different static mixers is given by Kale et al. [6,7]; however Xu et al. [32], use specific values for the rheological properties of a CMC solution. Also, pressure drop across a static mixer in turbulent flow regimes is studied $[3,4]$. The majority of correlations make use of a Z-factor approach. The Z-factor is a non-dimensional pressure drop, which is obtained by dividing the pressure drop across the mixer by the pressure drop in a pipe having the same diameter with no mixer. The Zfactor is presented as a function of the Reynolds number. Heywood, et al. [21] provide a review containing various functional dependencies of the Z-factor on Reynolds number and 
their theoretical basis. Wilkinson and Cliff [33] proposed a correlation between Reynolds number and pressure drop ratio for Kenics mixers as follows:

$$
\mathrm{Z}=7.19+0.03125 \mathrm{Re}
$$

Heat Transfer: A number of studies have considered heat transfer in different static mixers [8,29,34-37]. Joshi et al. studied wall to tube heat and mass transfer in a Kenics static mixer and recommended that a compact mixing element [34] can improve the rate of heat transfer. Li et al [35] studied pressure drop, heat transfer, and mixing mechanisms in a Sulzer SMX static mixer. Based on their experimental results, a general heat transfer coefficient correlation in a Sulzer SMX mixer shows an enhancement of up to a factor of five compared to a tube with no mixing element [35]. For laminar flow, [11,38,39] suggest correlations which are based on modifications of the Seider-Tate equation for laminar flow in an empty pipe. These correlations are written as follow, respectively:

$$
\begin{gathered}
\mathrm{Nu}=3.65+3.8\left(\operatorname{Re} \operatorname{Pr} \frac{D}{l}\right)^{1 / 3} \\
\mathrm{Nu}=4.65\left(\operatorname{Re} \operatorname{Pr} \frac{D}{l}\right)^{1 / 3} \\
\mathrm{Nu}=1.86\left(\operatorname{Re} \operatorname{Pr} \frac{D}{l}\right)^{1 / 3}
\end{gathered}
$$


For turbulent flow in a static mixer, analogous equations were presented $[8,38]$, using an exact threefold multiplication of the Seider-Tate heat transfer equation for a turbulent flow. Mass Transfer: A number of studies investigated mass transfer away from the walls of a tube equipped with helical static mixer elements. For laminar flow, Morris and Misson [9] suggest following correlation for the mass-transfer:

$$
\operatorname{Sh}=1.86(1+0.32 N)\left(\operatorname{ReSc} \frac{D}{l}\right)^{1 / 3}
$$

$N$ is the number of mixing elements. The element aspect ratio $\left(\frac{L}{D}\right)$ is 2.5 .

For turbulent flow in a static mixer, a two to three fold improvement for mass transfer in the Kenics mixer vs. an empty tube was reported, with the maximum relative improvement in the transitional flow regime $(\operatorname{Re}=1,000-4,000)$ [8].

Droplet Dispersion: The performance of static mixers has been investigated for dispersion of immiscible liquids when the flow regime is turbulent. Experimental studies of droplet break-up have been performed for different mixers [12-14]. Based on these experiments, correlations have been developed which relate the mean drop size in terms of the Sauter mean diameter $\left(D_{32}\right)$ to the Weber number (We) and the Reynolds number $(\mathrm{Re})$. Berkman and Calabrese [14] correlated their results for mean drop size as a function of Weber number and a viscosity group (Vi) as:

$$
\frac{D_{32}}{D}=\mathrm{AWe}^{-\frac{3}{5}}\left[1+1.38 \mathrm{Vi}\left(\frac{D_{32}}{D}\right)^{1 / 3}\right]^{3 / 5}
$$


Striation Thickness: Another concept that has been applied as a measure of mixing performance for static mixers in laminar flow is reduction of striation thickness during the blending of two initially segregated fluids. One experimentally obtained figure that presents perhaps the first fundamental study on the mixing in a helical mixer is shown in Fig. 2.1. In this visualization experiment, a two-part epoxy (one part black, the other white) is forced through the mixer and allowed to harden. The mixer is then cut into sections revealing the flow and mixing pattern at various axial locations along the mixer. It is unclear where the study originated ort first published. The figure appears in many publications, e.g., [22,23,40]. Avalosse and Crochet B1] recently performed a similar experiment using colored clay instead of epoxy.

Visual inspection of Fig. 2.1 also encouraged the first theoretical estimate of the mixing performance in a helical static mixer. Each element splits the flow in two. Thus, after two elements, there are four layers. After three elements, there are eight layers, and so onleading to the following formula:

$$
N_{L}=2^{N}
$$

Similar formulas have also been reported for other commercial mixers, see for example [41]. According to Eq. (2.7), twenty elements would produce nearly two million layers. In fact, for large numbers of elements the concept breaks down. After 30 elements in a $50 \mathrm{~mm}$ 
diameter pipe, the width of the layers would be $50 \mathrm{pm}$ wide, which is a width that is smaller than any single carbon atom in the epoxy.

Despite this conceptual difficulty, the mixing-layer or striation concept has been a useful mixing index in several studies. Most recently, Jaffer and Wood [42] studied the effect of flow ratio and viscosity ratio on the mixing in a helical static mixer $(4 \leq \operatorname{Re} \leq 125)$. The elements studied were different from the standard helical design. The elements had a twist ratio of $90^{\circ}$ and an element aspect ratio between 0.8 and 1.5. The number of elements $(N)$ was varied from 1 to 6 .

From digitized photographs of the dyed fluid leaving the mixer, they measured the average striation width, the variation of striation width, and the interfacial area between the dyed and un-dyed fluids leaving the mixer.

This information resembles most other mixing indices. However, Jaffer and Wood [42] also describe information of a more fundamental nature obtained using laser-induced fluorescence (LIF) to visualize the flowfield within the static mixer. Based on their observations, Jaffer and Wood report the formation of vortices on the top and bottom of the first mixing element at $\operatorname{Re}=55$ and $\operatorname{Re}=43$ for element aspect ratios of 1.0 and 0.8 , respectively. For the same element aspect ratios, vortices form at the second element at $\operatorname{Re}$ $=105$ and $\operatorname{Re}=90$, respectively. The lower critical Re for the shorter elements indicates that they tend to produce a stronger transverse motion. The gross-mixing indices did not improve with the appearance of the vortices. 


\subsection{Numerical Modeling of Static Mixers}

\subsubsection{Early Efforts Using CFD}

Several Early efforts at CFD modeling of the flow within static mixers were reported in the 1980's. Shintre and Ulbrecht [43] developed a Navier-Stokes-based model for the flow within two sheets of a Koch-Sulzer SMX mixer for $\operatorname{Re}^{\sim} 0$ and 1. Shintre [44] also extended the model to pseudo-plastic fluids.

For helical static mixers, several groups of researchers 24-27,45,46] tried to take advantage of the natural helical geometry of the mixer to simplify the Navier-Stokes equations. Each study reports transforming reduced forms of the Navier-Stokes equations into non-orthogonal, helical coordinates. Interestingly, each study claims that the previous one made a mistake in the coordinate transformation, which shows the difficulty of this approach. Despite the differences between each study and refinements in the details of the numerical methods used, the studies were limited to creeping flows $\left(\operatorname{Re}^{\sim} 0\right)$ or in the case of Dackson and Nauman [25] to $\operatorname{Re}<10$ and to the assumption of fully developed flow within the mixer. The paper by Arimond and Erwin [24] is notable because even though their flow solution was based on a fully developed assumption, they recognized the importance of the end effects and attempted flow to account for them.

Each of the studies mentioned in the previous sections focused on fully developed flow within a single mixer element. Nauman [20] demonstrated that this assumption is seriously flawed because it violates the conservation of mass; residence time distributions computed from these flow fields have an infinite mean. Moreover, recent experiments on the PPM 
flow suggest that flow in this idealized mixer is highly three-dimensional and dominated by entrance and exit effects [2]. Studies that use modern CFD methods account for both threedimensionality and entrance and exit effects. The next sections review recent modeling efforts using CFD codes.

\subsubsection{Recent CFD Modeling}

Some of the earliest efforts using a commercial CFD code were reported by Tanguy and coworkers $[30,47,48]$ for Newtonian and non-Newtonian flow through several different static mixers at low Re. Bakker and LaRoche [28] were the first to report results obtained by using a modern CFD code for the helical static mixer. Avalosse and Crochet [31] reported using a finite-element code to model the fluid flow in a mixer with an infinite number of mixer elements, which enabled them to use periodic boundary conditions in the longitudinal direction; an assumption that is only slightly better than the fully developed flow assumption of the pre-CFD studies. However, the most extensive studies were performed within the PhD dissertations of Byrde [49], Hobbs [50] and Jones [51]. Also, an investigation by Tanguy et al. [52] of the mixing performance of five different static mixers using a commercial CFD code falls within this category.

Hobbs and Muzzio: The primary objective of Hobbs' research [50] was to extend techniques which are used to study chaotic advection. These techniques have been widely used in order to study mixing in simple, two-dimensional flows [53]. Moreover, they have been used to study a realistic, three-dimensional, industrial mixer. The primary focus in Hobbs and Muzzio's research is the mixing performance of the helical mixer as computed 
by these techniques. Their work was not indented to predict the flowfield in the mixer. They used the commercial code FLUENT to predict the fluid flow in a helical static mixer. All mixing studies were based on the computed flowfield for a six-element mixer. A tetrahedral unstructured computational mesh containing 350,000 cells was used. A twodiameter long section of empty pipe was provided upstream and downstream of the mixing elements. A uniform velocity profile was used at the flowfield inlet. On the pipe wall and element surfaces, the no-slip boundary condition was used. At the flowfield outlet, a uniform pressure was specified for the entire cross-section [54]. Mixing studies were based on computing the trajectory of zero-mass particles in the computational flowfield. The summary of conclusions of this study is as follows;

- For creeping flows, mixing in the helical mixer is globally chaotic as long as the direction of twist alternates from mixing element to mixing element $[55,56]$ and the twist angle is greater than $60^{\circ}[56]$.

- Based on simulations at $\operatorname{Re}=015$, the most efficient mixing element design is based on a twist angle of $120^{\circ}$. The performance of the helical mixer was insensitive to element aspect ratio suggesting that shorter elements are more efficient [56].

- For Reynolds numbers around 100, the flow contains a mixture of chaotic and regular flow regions, and, thus, the mixer should not be used in this range of flows. Around Reynolds number 1000, the flow again appears globally chaotic [57].

- The velocity fields are essentially invariant for $0.15 \leq \operatorname{Re} \leq 10$ [54]. 
- Optimum injection sites are near the location of the manifolds of periodic points [54].

Byrde and Sawley: The primary objective of Byrde and Sawley's study was to develop a computational technique to compute mixing and flowfields using massively parallel supercomputers. A large portion of their work focused on the computational details of efficiently distributing the computational work load over many processors, that is, between 256 processors [58,59]. The flowfield calculations were mainly performed with a finitevolume solver, which uses the artificial compressibility method to couple velocity and pressure. The solver uses an alternating-direction implicit (ADI) scheme to solve the Navier-Stokes equations. Convective terms were discretized using a MUSCL-based, second-order, upwind ?-scheme combined with an approximate Roe method. The solver uses a multi-block-structured computational grid, which allows it to exploit the massively parallel computer architecture of a Cray machine and similar computers. Because the solver performed poorly for low Reynolds number conditions, Byrde and Sawley [60] used FLUENT software for low Reynolds number flows. A Lagrangian approach was used to examine the mixing process [49].

A one and half-diameter long section of empty pipe was provided upstream and downstream of the mixing elements. At the flowfield inlet, a uniform velocity profile was specified. At the flow outlet, constant pressure was specified over the entire cross-section.

Byrde and Sawley used four different mesh densities for a six-segment mixer: 122,880, $376,320,929,280$, and 1,505,280 cells. They used an Htype mesh in the core of the pipe and an O-type mesh near the pipe wall [61]. The problem with using an O-type mesh is an 
unnecessary concentration of small cells near the center of the pipe. Byrde and Sawley concluded that:

- Computational grids with $1,500,000$ nodes are required to obtain flowfields that obtain grid-independent particle trajectories based on structural radius [61].

- Comparisons of computed Poincarè sections indicated global chaos for $\mathrm{Re}=0.02$ and $\operatorname{Re}=200$, but distinct unmixed islands at $\operatorname{Re}=50$ [62].

- Based on the parametric studies the mixer performance is the greatest for a Reynolds number of 320 . At $\operatorname{Re}=200$, a twist angle of $180^{\circ}$ gives the most efficient mixing.

The work of Hobbs and Muzzio 57] and Byrde and Sawley [60] represent two substantial and important studies in the effort to correctly predict and understand the mixing behavior of helical static mixers. However, the conclusions of their work are contradictory in several important respects. The most glaring contradiction is that Hobbs and Muzzio report that mixing is the poorest for Reynolds numbers of the order of 100 , whereas, Byrde and Sawley determine that it is the most efficient for these Reynolds numbers. Further, Byrde and Sawley claim that the grid density needed to obtain physically realistic results are far in excess of the grid density used by Hobbs and Muzzio. Since neither study fully investigated the predicted flow fields, both overlooked potentially important and useful information. One particular point of agreement between the studies of Hobbs and Muzzio and Tanguy and coworkers is that, for creeping flows, the most stretching, and therefore, the most mixing occurs at the intersection of the mixing elements. 
This result demonstrates the importance CFD-based studies and the inadequacy of those earlier studies that ignored the developing flows within the mixer elements.

In the literature, only a few modeling studies of turbulent flow in static mixers have been reported [29,63-65]. Note that in a very early modeling effort, Date [66] developed a two-dimensional model for the laminar and turbulent flow in a pipe containing a twistedtape insert. The reduced Navier-Stokes equations were transformed to use stream function and vorticity as dependent variables. Turbulent flow was modeled using an early version of the $k-\varepsilon$ model. The model predictions for the friction factor were an order of magnitude lower than the then-available experimental correlations. Nonetheless, this study represents perhaps the earliest application of CFD to helical pipe geometries.

In two brief communications [28,64], Bakker and coworkers report using the commercial CFD code FLUENT to model the flow in Chemineer's HEV static mixer. The flow at a $\operatorname{Re}$ of 100,000 was predicted using the $k-\varepsilon$ turbulence model for the initial guess and the RSM turbulence model in the final predictions. A computational grid of 100,000 nodes was used. Only one-eighth of the mixer cross-section was modeled incorporating one-half of one mixer element cross-section. The inlet section in front of the first element was 0.5 diameters long and downstream of the second and last element was 2.5 diameters long. No information was given about the boundary conditions. The Quick scheme [67] was used for the convective terms. Also, no detail is given for the convergence properties of the code nor was any attempt made to investigate the effect of grid resolution on the predictions reported. Based on their predictions and the experiments of Gretta [68] and Fasano [69], it was concluded that optimal mixing occurs when chemicals are injected 
near the mixer elements and not in the center of the pipe. They also report that the calculations show small regions of reverse axial flow that could cause some axial dispersion within the mixer.

In a more detailed study [29,66-70], the commercial CFD code TASCflow was used to predict turbulent flow in a Koch-Sulzer SMV mixer. The particular application of interest was to use the static mixer for denitrification in an air pollution control facility. The mixer in this study was mounted within a rectangular duct. The Reynolds number was 426,000. Only one mixer element was used. The mixer geometry was simplified by assuming that the sidewalls of the duct do not affect the flow field and, thus, periodic boundary conditions were used in the direction perpendicular to the longitudinal flow. The grid consisted of a total of 105,575 nodes. The $k-\varepsilon$ turbulence model was used. Wall functions were used for boundary conditions on the walls of the duct. No study was made of the effect of grid density. The mixing process was modeled by solving the energy equation for the mean temperature of the fluid. By modifying the coefficient and neglecting dissimilar terms, the energy equation for incompressible flow can be reduced to the advection-dispersion equation for mean concentration.

The results of the model predictions were compared with limited LDV measurements in a 1:14-scale laboratory model of the mixer. The agreement between the model predictions and the experimental measurements for the longitudinal mean velocity and the mean temperature was largely unsatisfactory. The predictions did not capture the shape of the measured-mean velocity or mean temperature profiles. The predictions were also 
unable to reproduce the measured intensity of segregation downstream of the mixer element.

Jones, Sotiropoulos, and Amirtharajah: One of the most recent studies on helical static mixers have been done by Jones et al. [63] for water treatment. The model they used solves the three-dimensional, Reynolds-averaged Navier-Stokes equations, closed with the $k-\omega$ turbulence model, using a second order finite volume method. The model is applied to a two-element helical static mixer. The Reynolds number is 100,000, which is within the range of Reynolds numbers that is relevant to water treatment plants. The predicted flowfield is used to examine existing theories of rapid mixing and explore various approaches for characterizing mixing in static mixers for water treatment applications. Jones et al, [63] used a 1,035,125 cell structured mesh for a two-segment mixer with a long pipe and elbow prior to the mixing elements. Neither the mesh type, the mesh generation algorithm, nor the mesh density are not mentioned; however, unnecessary small cells can be seen in the computational domain.

It was concluded that in the turbulent flow regime in the helical static mixer that they considered, for $5,000 \leq \operatorname{Re} \leq 20,000$ the mixer elements appear to stabilize the incoming, fully developed, turbulent pipe flow yielding an almost laminar flow at the exit of the mixer. This laminarization depends strongly on the number of elements where more elements tend to stabilize the flow more strongly [51].

Also, they concluded that increasing the Reynolds number from 100 to 500 has a dramatic effect on the complexity of the flow in a two-element mixer. It intensifies and complicates the structure of the transverse motion, which has a profound effect on the 
cross-sectional distribution of the longitudinal momentum. More specifically, at $\operatorname{Re}=100$ the contours of longitudinal velocity at the start of the second element exhibit a flower-like pattern with a four-fold circumferential symmetry. On the other hand at $\operatorname{Re}=500$, the flow at the start of the second element exhibits six peaks with only the anti-diametrically located quadrants being symmetric [51].

They observed pockets of reversed flow near the leading and trailing edges of the mixer elements. These pockets appear at the junction of the element and the pipe wall, presumably due to locally-induced adverse pressure gradients. These reversed-flow regions were observed in both the laminar and turbulent flow simulations, but their size depends strongly on the Reynolds number. Their size was found to shrink substantially, compared to lower Reynolds number flowfields, at the highest turbulent Reynolds number simulated $(\operatorname{Re}=100,000)$ in their research [51].

What distinguished this study from the works done by Hobbs [57] and Byrde $[60,61]$ is that high Reynolds number flows were considered in helical static mixers. However, the geometry considered in this case is relatively a simple one and they only considered two mixing elements. Table 2.1 summarizes some the most recent numerical studies on static mixers. 


\begin{tabular}{|c|c|c|c|c|}
\hline Researcher(s) & $\begin{array}{c}\text { Mesh size } \\
\text { (cells) }\end{array}$ & Flow regime & Fluid type & Heat Transfer \\
\hline $\begin{array}{c}\text { Hobbs and } \\
\text { Muzzio }\end{array}$ & 350,000 & $\begin{array}{c}\text { Creeping and } \\
\text { laminar }\end{array}$ & Newtonian & Not considered \\
\hline $\begin{array}{c}\text { Byrde and } \\
\text { Sawley }\end{array}$ & $\begin{array}{c}\text { From 122,880 } \\
\text { up to 1,505,280 }\end{array}$ & $\begin{array}{c}\text { Creeping and } \\
\text { laminar }\end{array}$ & Newtonian & Not considered \\
\hline $\begin{array}{c}\text { Jones, } \\
\text { Sotiropoulos, } \\
\text { and } \\
\text { Amirtharajah }\end{array}$ & $1,035,125$ & Turbulent & Newtonian & Not considered \\
\hline $\begin{array}{c}\text { Rahmani, } \\
\text { Keith, and } \\
\text { Ayasoufi }\end{array}$ & $\begin{array}{c}\text { From 446,489 } \\
\text { up to 3,756,070 }\end{array}$ & $\begin{array}{c}\text { Creeping, } \\
\text { laminar, and } \\
\text { turbulent }\end{array}$ & $\begin{array}{c}\text { Newtonian and } \\
\text { non-Newtonian }\end{array}$ & Considered \\
\hline
\end{tabular}

Table 2.1: Summery of recent numerical studies on static mixers 


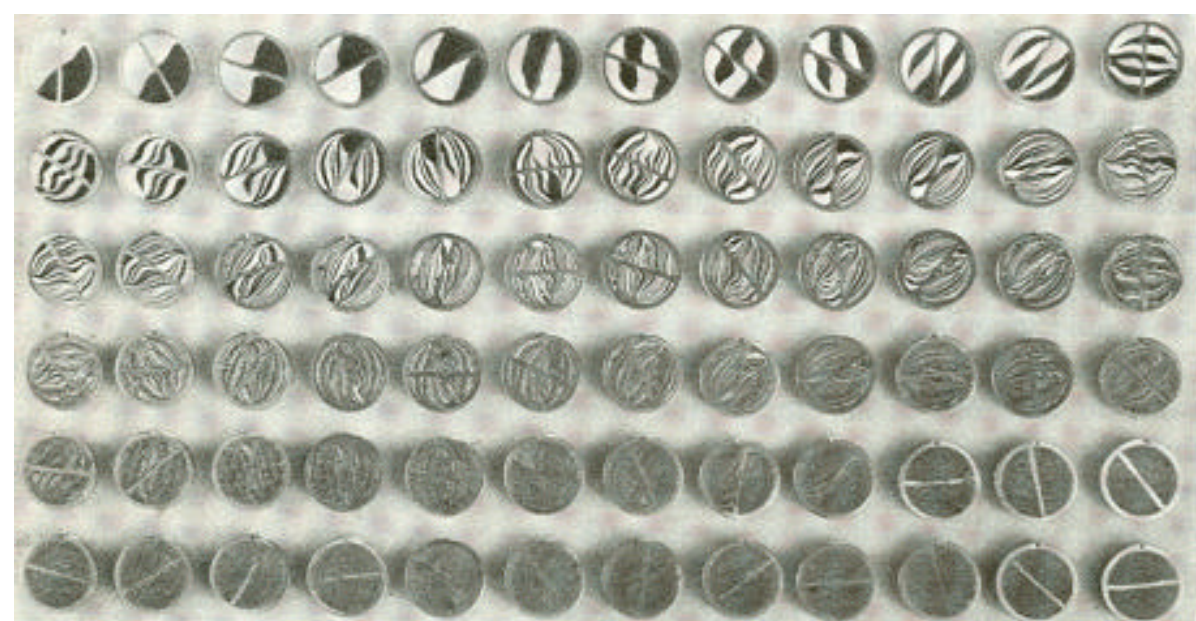

Figure2.1: Progressive mixing in a helical static mixer (Kenics mixer) 


\section{CHAPTER THREE}

\section{ANALYSIS AND NUMERICAL METHOD}

Governing equations of incompressible flow in static mixer is presented. A brief explanation of main aspects of non-Newtonian fluids and also description of the RANS turbulence models and Large-Eddy Simulation is presented in this chapter.

\subsection{Incompressible Flows}

For an unsteady incompressible flow, the mass conservation equation can be written as:

$$
\frac{\partial u_{i}}{\partial x_{i}}=0
$$

and the momentum conservation equation can be written as:

$$
\rho \frac{\partial u_{i}}{\partial t}+\rho \frac{\partial\left(u_{i} u_{j}\right)}{\partial x_{j}}+\frac{\partial p}{\partial x_{i}}=\frac{\partial \tau_{i j}}{\partial x_{j}}+\rho g_{i}+F_{i}
$$


For a steady flow, Eq. (3.2) becomes:

$$
\rho \frac{\partial\left(u_{i} u_{j}\right)}{\partial x_{j}}+\frac{\partial p}{\partial x_{i}}=\frac{\partial \tau_{i j}}{\partial x_{j}}+\rho g_{i}+F_{i}
$$

In the absence of a gravitational body force and any external body force, the last two terms on the right side of Eq. (3.3) are zero. The stress tensor, $\tau_{i j}$, in Eq. (3.3) is given by

$$
\tau_{i j}=\mu\left(\frac{\partial u_{i}}{\partial x_{j}}+\frac{\partial u_{j}}{\partial x_{i}}\right)-\frac{2}{3} \mu \frac{\partial u_{k}}{\partial x_{k}} \delta_{i j}
$$

It should be wted that for an incompressible steady flow, the conservation of mass makes the last term of Eq. (3.4) zero. In Eq. (3.4), $\mu$ is the molecular viscosity. For Newtonian fluids, $\mu$ is independent of the strain rate of the flow. For non-Newtonian fluids, viscosity becomes a function of the strain rate.

\subsection{Heat Transfer}

The energy equation for a steady incompressible flow is

$$
\frac{\partial\left(\rho u_{i} E+p\right)}{\partial x_{i}}=\frac{\partial}{\partial x_{i}}\left(K \frac{\partial T}{\partial x_{i}}+u_{j} \tau_{i j}\right)
$$


The total energy in Eq. (3.5) is

$$
E=h-\frac{p}{\rho}+\frac{u_{i} u_{i}}{2}
$$

For a pure material with no phase change the enthalpy is given by

$$
h=\int_{0}^{T} c_{p} d T
$$

For the material used in this study, water, the value of density is $998.2 \mathrm{~kg} / \mathrm{m}^{3}$. Although the specific heat is a function of temperature, for the temperature range used in this study its changes can be neglected; hence, specific heat is set at $4182 \mathrm{~J} / \mathrm{kg} . \mathrm{K}$. However, for the thermal conductivity, $K$, and the viscosity, $\mu$, the change of their values based on the temperature is significant and has an impact on the results of the numerical simulation. Therefore, the thermal conductivity and the viscosity are assumed piecewise linear functions of the temperature using the values from [71]. 


\subsection{Non-Newtonian Fluids}

Details about different models for non-Newtonian viscosity can be found in the literature, e.g., [72-74]. Here, after an introduction to non-Newtonian fluids, two of the most common models that are used in numerical studies are briefly explained.

The Newtonian viscosity depends only on temperature and pressure; it is independent of the rate of shear. The diagram relating shear stress and rate of shear for Newtonian fluids, the so-called flow curve, is therefore a straight line of slope $\mu$, and the single constant, $\mu$, completely characterizes the dynamic viscosity of the fluid. Fluids in which the dissipation of viscous energy is due to the collision of comparatively small molecular species manifest Newtonian behavior. All gases and liquids and solutions of low molecular weight fall into this category. Notable exceptions are colloidal suspensions and polymeric solutions where the molecular species are large. These fluids show obvious deviations from Newtonian behavior.

In contrast, non-Newtonian fluids are those for which the flow curve is not linear, i.e., the viscosity of a non-Newtonian fluid is not constant at a given temperature and pressure but depends on other factors such as the rate of shear in the fluid, the apparatus in which the fluid is contained or even on the previous history of the fluid. Non-Newtonian fluids can be classified into three broad types:

- Fluids in which the rate of shear at any point is some function of the shearing stress at that point and depend on nothing else, 
- Fluids in which the relation between shear stress and shear rate depend on the time that the fluid has been sheared or on its previous history, and

- Materials which have characteristics of solids and fluids and exhibit partial elastic recovery after deformation, the so-called viscoelastic fluids.

The following describe these three types briefly.

\subsubsection{Time-Independent Non-Newtonian Fluids}

Non-Newtonian fluids of the first type are fluids with properties that are independent of time. For these materials, the rate of shear at any point in the fluid is a simple function of the shear stress at that point, when the temperature is constant. These fluids are called non-Newtonian viscous fluids. These fluids may conveniently be subdivided into three

distinct types depending on the nature of the function which relates the rate of shear to the shear stress:

- Bingham plastics

- Pseudo-plastic fluids

- Dilatant fluids

Typical flow curves for these three fluids are shown in Fig. 3.1 and compared with the linear relation typical of Newtonian fluids.

Bingham Plastics: A Bingham plastic is characterized by a flow curve which is a straight line having an intercept $\tau_{y}$ on the shear-stress axis. The yield stress, $\tau_{y}$ is the stress which must be exceeded before flow starts. The rheological equation for a Bingham is: 


$$
\tau-\tau_{y}=\mu_{p} \gamma ; \tau>\tau_{y}
$$

where $\mu_{p}$ is the slope of the flow curve and called the plastic viscosity or the coefficient of rigidity. The explanation of Bingham plastic behavior is that the fluid at rest contains a three-dimensional structure of sufficient rigidity to resist any stress less than the yield stress, $\tau_{y}$. If this stress is exceeded the structure completely disintegrates and the system behaves as a Newtonian fluid under a shear stress $\tau-\tau_{y}$. When the shear stress falls below the yield stress, the structure is reformed. The concept of an idealized Bingham plastic is very convenient in practice because many real fluids closely approximate this type of behavior. Common examples of Bingham plastics are slurries, drilling muds, oil paints, toothpaste and sewage sludges.

Pseudo-Plastic Fluids: A pseudo-plastic fluid has no yield stress $\left(\tau_{y}=0\right)$. The flow curve for these materials indicates that the ratio of shear stress to the rate of shear, the apparent viscosity, falls progressively with shear rate and the flow curve becomes linear only at very high rates of shear. This limiting slope is known as the viscosity at infinite shear. The logarithmic plot of shear stress and rate of shear for these materials is often found to be linear with a slope between zero and unity. As a result, an empirical functional relation known as the power law is widely used to characterize fluids of this type. This relation was originally proposed by Ostwald [73] and has since been fully described by Reiner [75]. When the temperature is constant, the power law relation can be written as follows: 


$$
\tau=\kappa \dot{\gamma}^{n}
$$

$\kappa$ and $n$ are constants $(n<1)$ for a particular fluid. $\kappa$ is a measure of the consistency of the fluid; the larger $\kappa$ the more viscous the fluid. $n$ is a measure of the degree of nonNewtonian behavior, and the greater the departure from unity the more pronounced are the non-Newtonian properties of the fluid. It should be mentioned that although $n$ is nearly constant in many cases over wide ranges of shear rate it is not a true constant for real fluids over all possible ranges of shear. This is not a serious drawback in engineering applications because all that is needed is a rheological equation which describes the fluid over the particular range of shear rate encountered in the particular problem. Over such a range $n$ may often be regarded as constant.

The apparent viscosity for a power law fluid can be expressed in terms of $n$ as:

$$
\mu=\frac{\tau}{\dot{\gamma}}=\kappa \dot{\gamma}^{n-1}
$$

A pseudo-plastic fluid viscosity can be defined for different temperatures as:

$$
\mu=\kappa e^{\frac{T}{T_{0}}} \gamma^{n-1}
$$

where $T_{0}$ is a reference temperature, which is generally taken to be $25^{\circ} \mathrm{C}$. It should be mentioned that since $n<1$ for pseudo-plastics the apparent viscosity decreases as the rate 
of shear increases. This is the reason that pseudo-plastic fluids are also called shearthinning fluids. This type of behavior is characteristic of suspensions of asymmetric particles or solutions of high polymers such as cellulose derivatives. This suggests that the physical interpretation of this phenomenon is probably that with increasing rates of shear the asymmetric particles or molecules are progressively aligned, instead of the random intermingled state which exists when the fluid is at rest the major axes are brought into line with the direction of flow. The apparent viscosity continues to decrease with increasing rate of shear until no further alignment along the streamlines is possible and the flow curve then becomes linear.

Pseudo-plastic fluids have been defined as time-independent fluids and this implies that the alignment of molecules suggested above takes place instantaneously as the rate of shear is increased or, at any rate, so quickly that the time effect cannot be detected using ordinary viscometric techniques.

In the literature other empirical equations can be found which describe the behavior of pseudo-plastic fluids. A sampling of these relations are:

$$
\begin{aligned}
\tau & =C_{1} \sin ^{-1}\left(\frac{\gamma}{C_{2}}\right) \\
\tau & =\frac{\gamma}{C_{3}}+C_{2} \sin \left(\frac{\tau}{C_{1}}\right) \\
\tau & =C_{1} \dot{\gamma}+C_{3} \sinh ^{-1}\left(C_{2} \dot{\gamma}\right) \\
\tau & =\frac{C_{1} \gamma}{\left(C_{3}+\gamma\right)}+\mu_{\infty} \dot{\gamma}
\end{aligned}
$$


These equations are called the Prandtl, the Eyring, the Powell-Eyring, and the Williamson equations, respectively. $C_{1}, C_{2}$ and $C_{3}$ in the equations are constants which are typical of the particular fluid. These equations are considerably more difficult to use than the power law and usually do not offer any compensating advantages [73].

Another equation that is used to describe pseudo-plastic fluids is called the Carreau model. The Carreau model attempts to describe a wide range of fluids by the establishment of a curve-fit to piece together functions for both Newtonian and shearthinning non-Newtonian laws. The Carreau model uses the following equation for the viscosity:

$$
\mu=\mu_{\infty}+\left(\mu_{0}-\mu_{\infty}\right)\left[1+\left(\lambda e^{\frac{T}{T_{0}}} \gamma\right)^{2}\right]^{\frac{n-1}{2}}
$$

Dilatant Fluids: A dilatant fluid is similar to pseudo-plastics in that they show no yield stress but the apparent viscosity for these materials increases with increasing rates of shear. These materials are also called shear-thickening fluids. The power law equation is again often applicable but in this case the index $n$ is greater than unity. This type of behavior was originally found in suspensions of solids at high solids content by Osborne Reynolds. He suggested that when these concentrated suspensions are at rest the voidage is at a minimum and the liquid is only sufficient to fill these voids. When these materials are sheared at low rates the liquid lubricates the motion of one particle past another and the stresses are consequently small. At higher rates of shear the dense packing of the 
particles is broken up and the material expands or dilates slightly and the voidage increases. There is now insufficient liquid in the new structure to lubricate the flow of the particles past each other and the applied stresses have to be much greater. The formation of this structure causes the apparent viscosity to increase rapidly with increasing rates of shear. The term dilatant has since come to be used for all fluids which exhibit the property of increasing apparent viscosity with increasing rates of shear. Many of these, such as starch pastes, are not true suspensions and do not dilate on shearing in the normal sense of the word. The above explanation therefore does not apply but nevertheless they are commonly referred to as dilatant fluids. It is mentioned that, in process industries dilatant fluids are much less common than pseudo-plastic fluids but when the power law is applicable the treatment of both types is much the same.

\subsubsection{Time-Dependent Non-Newtonian Fluids}

Many real fluids cannot be described by a simple rheological equation such as Eq. (3.9), which applies to fluids for which the relation between shear stress and shear rate is independent of time. The apparent viscosity of more complex fluids depends not only on the rate of shear but also on the time the shear has been applied. Accordingly, as the shear stress decreases or increases with time when the fluid is sheared at a constant rate, these fluids are subdivided into two classes:

- Thixotropic fluids

- Rheopectic fluids 
Thixotropic Fluids: Thixotropic materials are those whose consistency depends on the duration of shear as well as on the rate of shear. If a thixotropic material is sheared at a constant rate after a period of rest, the structure will be progressively broken down and the apparent viscosity will decrease with time. The rate of breakdown of structure during shearing at a given rate will depend on the number of linkages available for this breaking and must therefore decrease with time. The simultaneous rate of reformation of structure will increase with time as the number of possible new structural linkages increases. Eventually a state of dynamic equilibrium is reached when the rate of building up of structure equals the rate of breakdown. This equilibrium position depends on the rate of shear and moves towards greater breakdown at increasing rates of shear. Thixotropy is a reversible process and after resting the structure of the material builds up again gradually.

Thixotropy is rather like pseudo-plasticity in which the time required for the alignment of particles is not negligible. This time effect for pseudo-plastic materials is not observable in the apparatus normally used for the testing of these fluids. The difference then is only a matter of degree.

Rheopectic Fluids: Rheopectic fluid is a non-Newtonian fluid in which its structure is formed by shear. For the other types of fluids considered previously, shearing tends to destroy their structure.

Freundlich and Juliusberger, [73], using a $42 \%$ gypsum paste in water, found that after shaking, this material re-solidified in 40 minutes if at rest; but it re-solidified in 20 seconds if the vessel was gently rolled in the palms of one hands. This seems to indicate that small shearing motions facilitate structure build-up but large shearing (shaking) destroys it. There is a critical amount of shear beyond which reformation of structure is 
not induced but breakdown occurs. This behavior is also observed with dilute aqueous solutions of vanadium pentoxide and bentonite.

There are other materials, however, in which structure only forms under shear and gradually disintegrates when at rest. This is usually termed rheopexy but it is quite distinct from the definition of this term given by Freundlich to the behavior of gypsum pastes. Even so this behavior is only found at moderate rates of shear, for if shearing is rapid the structure does not form. A $0.005 \mathrm{~N}$, where $\mathrm{N}$ denotes rotational speed, suspension of ammonium oleate behaves in this way. Consider the flow of this material through a capillary tube [73]. At a moderate pressure difference the flow is rapid at first and then decreases as the structure builds up. At a high pressure difference the flow is always rapid and does not fall off because the structure does not build up at high rates of shear.

Rheopectic fluids, e.g., ammonium oleate are superficially similar to their timeindependent counterparts (dilatant fluids) in which the time for structure build-up is insignificantly small. Here, however, the analogy is not so close because rheopexy is a case where build-up is brought about by small shearing rates only. There is an upper limit to the shear rate beyond which the analogy breaks down.

\subsubsection{Viscoelastic Fluids}

A viscoelastic material is one which possesses both elastic and viscous properties, i.e., although the material might be viscous, it exhibits a certain elasticity of shape. This concept is perhaps most easily visualized in the case of a very viscous liquid such as 
pitch. Consider the simplest case where we assume Newton's law for the viscous component and Hooke's law for the elastic component. In steady state flow under a shear stress $\tau$, the rate of shear will be $\frac{\tau}{\mu_{0}}$ where $\mu_{0}$ is a constant Newtonian viscosity coefficient. Now, suppose that the shear stress is increased to $\tau+d \tau$ very rapidly. The material will now be sheared through an additional angle $\frac{\delta \tau}{G}$ where $G$ is a rigidity modulus. There is therefore an additional rate of shear proportional to the rate of change of stress at any instant and the total rate of shear is given by:

$$
\dot{\gamma}=\frac{\tau}{\mu_{0}}+\frac{\dot{\tau}}{G}
$$

or it can be written as

$$
\tau+\lambda_{1} \tau=\mu_{0} \dot{\gamma}
$$

where $\lambda_{1}=\frac{\mu_{0}}{G}$. This equation was first proposed by Maxwell [74], and liquids which are described by it are usually referred to as Maxwell liquids. The parameter $\lambda_{1}$ has dimensions of time and it is seen from Eq. (3.15) that it is the time constant of the exponential decay of stress at a constant strain, i.e., if the motion is stopped the stress will relax as $\exp \left(-\frac{t}{\lambda_{1}}\right)$, Consequently $\lambda_{1}$ is known as the relaxation time. 
Oldroyd [74] investigated the elastic and viscous properties of emulsions and suspensions of one Newtonian liquid in another and derived theoretically the differential equation relating the shear stress $\tau$ and the rate of shear $\dot{\gamma}$ in the form

$$
\tau+\lambda_{1} \dot{\tau}=\mu_{0}\left(\dot{\gamma}+\lambda_{2} \ddot{\gamma}\right)
$$

where the constants $\mu_{0}, \lambda_{1}$, and $\lambda_{2}$ can be determined in terms of the physical properties of the mixture. In this system the elastic strain energy is stored during flow by virtue of the fact that interfacial tension provides the restoring force which makes the individual drops resist changes of shape. The same equation was also derived by Frolich and Sack [73] for a dilute suspension of solid particles in a viscous liquid. Elastic strain energy is stored because the solid elastic particles are deformed by the flow of the surrounding liquid.

In this equation the constant $\mu_{0}$ can be identified as the viscosity at low rates of shear in the steady state, i.e., when $\dot{\tau}=\ddot{\gamma}=0$. The constant $\lambda_{1}$ is a relaxation time and the physical significance of this is that if the motion is suddenly stopped the shear stress will decay as $\exp \left(-\frac{t}{\lambda_{1}}\right) \cdot \lambda_{2}$ is called a retardation time and has the significance that if all stresses are removed the rate of strain decays as $\exp \left(-\frac{t}{\lambda_{2}}\right)$. 
Toms and Strawbridge [73] found that the behavior of dilute poly-methylmethacrylate in pyridine can be described by means of this sort of expression. It also describes the behavior of some bitumens.

It is apparent, then, that a viscoelastic fluid cannot be characterized by a simple rheological equation of the form of $\dot{\gamma}=f(\tau)$. The essential difference is that the rheological equation contains the time derivatives of both $\tau$ and $\gamma$ in general. Details about viscoelastic materials and their modeling can be found in the literature, e.g., [74].

\subsection{Non-Newtonian Fluid Modeling}

The non-Newtonian fluid studied here is carboxymethyl cellulose (CMC) in water, which is a member of the pseudo-plastic fluids group, characterized by a progressively decreasing slope or shear stress versus shear rate. These fluids are termed shear thinning; the viscosity decreases with increasing velocity gradient. CMC is used primarily in foods, drugs and cosmetics as a viscosifier, emulsion stabilizer, and thickener to improve texture. The main applications of a technical grade of CMC are in textile warp sizing and in paper processing. CMC is also used in detergents as an antiredeposition agent, adhesives, latex paints and polishes. In the past, several studies have been performed to investigate $\mathrm{CMC}$ properties or have reported making use of CMC properties, e.g., [7679]. CMC solutions are known as power law fluids [80]. 
In this study, two models are considered: the power law model, and the Carreau model. The power law model for the shear stress uses Eq. (3.11) for the viscosity. The power law model predicts infinite viscosity when the shear rate goes to zero. The Carreau model resolves the problem by the establishment of a curve-fit to piece together functions for both Newtonian and pseudo-plastic non-Newtonian laws. The Carreau model uses Eq. (3.13) for the viscosity. Values of $\mu_{0}, \lambda, \kappa$, and $n$ used in Eqs. (3.11) and (3.13) for a CMC solution depends on the concentration level of CMC in water. The reference temperature is $25^{\circ} \mathrm{C}$, which is also the working temperature in this study. It may be worth mentioning that the temperature has a significant effect on the rheological parameters of CMC [81].

\subsection{Turbulence Modeling}

Studying helical static mixer performance under turbulent flow conditions is a main part of this research. Two different approaches have been employed in order to model the turbulence in the flow through a static mixer. In the first pproach RANS turbulence models have been considered. In the second approach an attempt to study the turbulent flow using a Large-Eddy Simulation (LES) has been made. The first approach is more an engineering approach which provides some useful data while $\mathfrak{t}$ needs relatively short simulation time and is achievable by using a relatively small amount of computational memory. The second approach however, demands much more computational time and memory; and of course provides much more detailed information regarding the helical 
static mixer performance under turbulent flow conditions. This section provides general information regarding turbulence modeling. Details about specific turbulent models employed in this research are given in Chapter 7.

Although there is no complete and sufficient definition of turbulent flow, it may be useful to describe its nature. Turbulent flows represent an intrinsically three-dimensional, time and space dependent phenomena, which have a large number of spatial degrees of freedom. They carry a wide range of vorticities from large- to small-scale, which have a random nature; and of course turbulent flow is a continuum phenomenon. Turbulent flows are characterized by fluctuating velocity fields. These fluctuations mix transported quantities of mass, momentum, and energy and in turn cause the transported quantities to fluctuate as well. These fluctuations can be of small scale and high frequency, therefore generally they are too expensive to simulate directly.

Turbulence modeling is one of three key elements in Computational Fluid Dynamics. Very precise mathematical theories have evolved for the other two key elements, viz., grid generation and algorithm development. By its nature (in creating a mathematical model that approximates the physical behavior of turbulent flows) far less precision has been achieved in turbulence modeling. This is not really a surprising event since our objective has been to approximate an extremely complicated phenomenon.

The field of turbulence modeling is, to some extent, a throwback to the days of Prandtl, Taylor, and von Karman who successfully devised engineering approximations and models describing complicated physical flows. Simplicity combined with physical insight seems to have been a common denominator of the work of these great men. Using their work as a gauge, an ideal model should introduce the minimum amount of 
complexity while capturing the essence of the relevant physics. This description of an ideal model serves as the main cornerstone of this section.

Aside from any physical considerations, turbulence is inherently three dimensional and time dependent. Thus, an enormous amount of information is required to completely describe a turbulent flow. Fortunately, we usually require something less than a complete time history over all spatial coordinates for every flow property. Thus, for a given turbulent flow application, we must pose the following question. Given a set of initial and/or boundary conditions, how do we predict the physically meaningful properties of the flow? What properties of a given flow are meaningful is generally dictated by the application. For the simplest applications, we may require only the skin friction and heattransfer coefficients. More esoteric applications may require detailed knowledge of energy spectra, turbulence fluctuation magnitudes and scales.

Certainly, we should expect the complexity of the mathematics needed for a given application to increase as the amount of required flow field detail increases. On the one hand, if all we require is skin friction for an attached flow, a simple mixing-length model might be sufficient. Such models are well developed and can be implemented with very little specialized knowledge. On the other hand, if we desire a complete time history of every aspect of a turbulent flow, only a solution to the complete Navier-Stokes equation will suffice. Such a solution requires an extremely accurate numerical solver and may require use of subtle transform techniques, not to mention vast computer resources. Most engineering problems fall somewhere between these two extremes.

Thus, once the question of how much detail we need is answered, the level of complexity of the model follows, qualitatively speaking. In the spirit of Prandtl, Taylor 
and von Karman, the conscientious engineer will strive to use as conceptually simple an approach as possible to achieve his ends. Overkill is often accompanied by unexpected difficulties that, in CFD applications, almost always manifest themselves as numerical difficulties!

\subsubsection{Comments on the Physics of Turbulence}

Before plunging into the mathematics of turbulence, it is worthwhile to first discuss physical aspects of the phenomenon. The following discussion is not intended as a complete description of this complex topic. Rather, we focus upon a few features of interest in engineering applications, and in construction of a mathematical model.

In 1937, Taylor and von Karman [82] proposed the following definition of turbulence: "Turbulence is an irregular motion which in general makes its appearance in fluids, gases or liquids, when they flow past solid surfaces or even when neighboring streams of the same fluid flow past or over one another." It is characterized by the presence of a large range of excited length and time scales. The irregular nature of turbulence stands in contrast to laminar motion, so called historically, because the fluid was imagined to flow in smooth laminae, or layers. Virtually all flows of practical engineering interest are turbulent. Turbulent flows always occur when the Reynolds number is large. For slightly viscous fluids such as water and air, large Reynolds number corresponds to anything stronger than a small swirl or a puff of wind. Careful analysis of solutions to the Navier-Stokes equation, or more typically to its boundary-layer form, show that turbulence develops as an instability of laminar flow. 
To analyze the stability of laminar flows, virtually all methods begin by linearizing the equations of motion. Although some degree of success can be achieved in predicting the onset of instabilities that ultimately lead to turbulence with linear theories, the inherent nonlinearity of the Navier-Stokes equation prevents a complete analytical description of the actual transition process, let alone the fully turbulent state. For a real, i.e., viscous, fluid, the instabilities result from interaction between the Navier-Stokes equation's nonlinear inertial terms and viscous terms. The interaction is very complex because it is rotational, fully three dimensional and time dependent.

The strongly rotational nature of turbulence goes hand-in-hand with its three dimensionality. Vigorous stretching of vortex lines is required to maintain the everpresent fluctuating vorticity in a turbulent flow. Vortex stretching is absent in twodimensional flows so that turbulence must be three-dimensional. This inherent three dimensionality means there are no satisfactory two-dimensional approximations and this is one of the reasons turbulence remained the most noteworthy unsolved scientific problem of the twentieth century (and perhaps it will remain unsolved in this century as well!).

The time-dependent nature of turbulence also contributes to its intractability. The additional complexity goes beyond the introduction of an additional dimension. Turbulence is characterized by random fluctuations thus making difficult a deterministic approach to the problem. Rather, we must use statistical methods. On the one hand, this aspect is not really a problem from the engineer's view. Even if we had a complete time history of a turbulent flow, we would usually integrate the flow properties of interest over time to extract time-averages. On the other hand, time averaging operations lead to 
statistical correlations in the equations of motion that cannot be determined a priori. This is the classical closure problem.

In principle, the time-dependent, three-dimensional Navier-Stokes equation contains all of the physics of a given turbulent flow. That this is true follows from the fact that turbulence is a continuum phenomenon. As noted by Tennekes and Lumley [83], "Even the smallest scales occurring in a turbulent flow are ordinarily far larger than any molecular length scale." Nevertheless, the smallest scales of turbulence are still extremely small. They are generally many orders of magnitude smaller than the largest scales of turbulence, the latter being of the same order of magnitude as the dimension of the object about which the fluid is flowing. Furthermore, the ratio of the smallest to the largest scales decreases rapidly as the Reynolds number increases. To make an accurate numerical simulation, i.e., a full time-dependent three-dimensional solution of a turbulent flow, all physically relevant scales must be resolved. While more and more progress is being made with such simulations, today's computers have insufficient memory and speed to solve any turbulent flow problem of practical interest. To underscore the magnitude of the problem, Speziale [84] noted that numerical simulations of turbulent pipe flow at a Reynolds number of 500,000 would require a computer 10 million times faster than a Cray Y/MP. However, the results are very useful in developing and testing turbulence models in the limit of low Reynolds number.

Turbulence consists of a continuous spectrum of scales ranging from largest to smallest, as opposed to a discrete set of scales. The nonlinearity of the Navier-Stokes equation leads to interactions between fluctuations of differing wavelengths and directions. The main physical process that spreads the motion over a wide range of 
wavelengths is vortex stretching. The turbulence gains energy if the vortex elements are primarily oriented in a direction in which the mean velocity gradients can stretch them. Most importantly, wavelengths that are not too small compared to the mean-flow width interact most strongly with the mean flow. Consequently, the larger-scale turbulent motion carries most of the energy and is mainly responsible for the enhanced diffusivity and attending stresses. In turn, the larger eddies randomly stretch the vortex elements that comprise the smaller eddies, cascading energy to them.

An especially striking feature of a turbulent shear flow is the way large bodies of fluid migrate across the flow, carrying smaller-scale disturbances with them. The arrival of these large eddies near the interface between the turbulent region and non-turbulent fluid distorts the interface into a highly convoluted shape. In addition to migrating across the flow, they have a lifetime so long that they persist for distances as great as 30 times the width of the flow [85]. Hence, the turbulent stresses at a given position depend upon upstream history and cannot be uniquely specified in terms of the local strain-rate tensor as in laminar flow.

\subsubsection{A Brief History of Turbulence Modeling}

A large portion of the work done on turbulence modeling involves the time-averaged Navier-Stokes equation. The origin of this approach dates back to the end of the $19^{\text {th }}$ century when Reynolds [86] published results of his research on turbulence. His pioneering work proved to have such profound importance for all future developments that we refer to the standard time-averaging process as one type of Reynolds averaging. 
The earliest attempts at developing a mathematical description of turbulent stresses sought to mimic the molecular gradient-diffusion process. In this spirit, Boussinesq [87] introduced the concept of an eddy viscosity. As with Reynolds, Boussinesq has been immortalized in turbulence literature. The Boussinesq eddy-viscosity approximation is so widely known that few authors find the need to reference his original paper.

Neither Reynolds nor Boussinesq attempted solution of the Reynolds-averaged Navier-Stokes equation in any systematic manner. Much of the physics of viscous flows was a mystery at that time; and further progress awaited Prandtl's discovery of the boundary layer in 1904. Focusing upon turbulent flows, Prandtl 88] introduced the mixing length (an analog of the mean-free path of a gas) and a straightforward prescription for computing the eddy viscosity in terms of the mixing length. The mixinglength hypothesis, closely related to the eddy-viscosity concept, formed the basis of virtually all turbulence-modeling research for the next twenty years. Important early contributions were made by several researchers, most notably by von Karman [89]. In modern terminology, we refer to a mixing-length model as an algebraic model or a zeroequation model of turbulence. By definition, an $n$-equation model signifies a model that requires solution of $n$ additional differential transport equations in addition to those expressing conservation of mass, momentum and energy.

To improve the ability to predict properties of turbulent flows and to develop a more realistic mathematical description of the turbulent stresses, Prandtl [90] postulated a model in which the eddy viscosity depends upon the kinetic energy of the turbulent fluctuations, $k$. He proposed a modelled differential equation approximating the exact 
equation for $k$. This improvement, on a conceptual level, takes account of the fact that the eddy viscosity is affected by where the flow has been, i.e., upon flow history. Thus was born the concept of the so-called one-equation model of turbulence.

While having an eddy viscosity that depends upon flow history provides a more physically realistic model, the need to specify a turbulence length scale remains. Since the length scale can be thought of as a characteristic eddy size and since such scales are different for each flow, turbulence models that do not provide a length scale are incomplete. That is, we must know something about the flow, other than initial and boundary conditions, in advance in order to obtain a solution. Such models are not worthless; in fact, those models have proven to be of great value in many engineering applications.

To elaborate a bit further, an incomplete model generally defines a turbulence length in a prescribed manner from the mean flow, e.g., the displacement thickness, $\delta^{*}$, for an attached boundary layer. However, a different length in this example would be needed when the boundary layer separates since $\delta^{*}$ may be negative. Yet another le ngth might be needed for free shear flows, etc. In essence, incomplete models usually define quantities that may vary more simply or more slowly than the Reynolds stresses (e.g. eddy viscosity and mixing length). Presumably such quantities are easier to correlate than the actual stresses.

A particularly desirable type of turbulence model would be one that can be applied to a given turbulent flow by prescribing at most the appropriate boundary and/or initial conditions. Ideally, no advance knowledge of any property of the turbulence should be 
required to obtain a solution. Such a model is defined as being complete. Note that this definition implies nothing regarding the accuracy or universality of the model, only that it can be used to determine a flow with no prior knowledge of any flow details.

An incomplete model generally defines a turbulence length in a prescribed manner from the mean flow, e.g., the displacement thickness, $\delta^{*}$. Kolmogorov [91] introduced the first complete model of turbulence. In addition to having a modeled equation for $\mathrm{k}$, he introduced a second parameter $\omega$ that he referred to as "the rate of dissipation of energy in unit volume and time." The reciprocal of $\omega$ serves as a turbulence time scale, while $\frac{\sqrt{k}}{\omega}$ serves as the analog of the mixing length. In this model, known as a $k-\omega$ model, $\omega$ satisfies a differential equation similar to the equation for $k$. The model is thus termed a two-equation model of turbulence. While this model offered great promise, it was virtually not applied for the next quarter century because of the unavailability of computers to solve its nonlinear differential equations.

Rotta [92] laid the foundation for turbulence models that obviate use of the Boussinesq approximation. He devised a plausible model for the differential equation governing evolution of the tensor that represents the turbulent stresses, i.e., the Reynoldsstress tensor. This approach is called second-order or second-moment closure. The primary conceptual advantage of second-order closure is the natural manner in which non-local and history effects are incorporated. Such models automatically accommodate complicating effects such as streamline curvature, rigid-body rotation, and body forces. This stands in stark contrast to eddy-viscosity models that fail to properly account for 
these effects. For a three-dimensional flow, a second-order closure model introduces seven equations, one for the turbulence scale and six for the components of the Reynoldsstress tensor. As with Kolmogorov's $k$ - $\omega$ model, second-order closure models awaited adequate computer resources.

Thus, by the early 1950's, four main categories of turbulence models had evolved, viz.,

- Algebraic (Zero-Equation) Models

- One-Equation Models

- Two-Equation Models

- Second-Order Closure Models

With the coming of the age of computers since the 1960's, further development of all four classes of turbulence models has occurred. The following overview lists a few of the most important modern developments for each of the four classes.

Algebraic or Zero-Equation Models: Van Driest [93] devised a viscous damping correction for the mixing-length model that is included in virtually all algebraic models in use today. Cebeci and Smith [94] refined the eddy-viscosity/mixing-length model to a point that it can be used with great confidence for most attached boundary layers. To remove some of the difficulties in defining the turbulence length scale from the shearlayer thickness, Baldwin and Lomax [95] have proposed an alternative algebraic model that enjoys widespread use.

One-Equation Models: Of the four types of turbulence models described above, the oneequation model has enjoyed the least popularity and success. Perhaps the most successful 
model of this type was formulated by Bradshaw, Ferriss and Atwell [96]. In the 1968 Stanford Conference on Computation of Turbulent Boundary Layers the best turbulence models of the day were tested against the best experimental data of the day [97]. Some researchers reported, of all the models used, the Bradshaw-Ferriss-Atwell model most faithfully reproduced measured flow properties [97]. There has been some renewed interest in one-equation models (c.f. Baldwin and Barth [98], Goldberg [99] and Spalart and Allmaras [100]), motivated primarily by the ease with which such model equations can be solved numerically, relative to two-equation models and second-order closure models.

Two-Equation Models: While Kolmogorov's $k$ - $\omega$ model was the first of this type, it remained in obscurity until the coming of the computer. By far, the most extensive work on two-equation models has been done by Launder and Spalding [101] and a continuing succession of students and colleagues. Launder's $k$ - $\varepsilon$ model, where $\varepsilon$ is proportional to the product of $k$ and $\omega$, is as well known as the mixing-length model and is the most widely used two-equation model. Even the model's demonstrable inadequacy for flows with adverse pressure gradient (c.f. Rodi and Scheuerer [102] and Wilcox [103] has done little to discourage its widespread use. With no prior knowledge of Kolmogorov's work, Saffman [104] formulated a $k-\omega$ model that enjoys advantages over $k$ - $\varepsilon$ model, especially for integrating through the viscous sub-layer and for predicting effects of adverse pressure gradient. Wilcox and Alber [105], Saffman and Wilcox [106], Wilcox and Traci [107], Wilcox and Rubesin [108], and Wilcox [103], for example, have pursued further development and application of $k$ - $\omega$ models. About 10 years ago, a new version 
of $k$ - $\omega$ model has been introduced by Menter [109]. This new version is a modified version of the original model of Wilcox [103]. One problem with the original $k$ - $\omega$ model is that it does not correctly predict the asymptotic behavior of the turbulence in near-wall regions, because of the fact that the eddy viscosity is much smaller than the molecular viscosity close to a solid surface. The Menter's $k$ - $\omega$ model overcomes this problem (see Chapter 7). As pointed out by Lakshminarayana [110], $k$ - $\omega$ models are the second most widely used type of two-equation turbulence model.

Second-Order Closure Models: By the 1970's, sufficient computer resources became available to permit serious development of this class of model. The most noteworthy efforts were those of Donaldson [111], Daly and Harlow [112] and Launder, Reece and Rodi [113]. The latter has become the baseline second-order closure model: more recent contributions by Lumley [114], Speziale [84,115] and Reynolds [116] have added mathematical rigor to the closure process. However, because of the large number of equations and complexity involved in second-order closure models, they have thus far found their way into a relatively small number of applications compared to algebraic and two-equation models.

\subsubsection{The Closure Problem}

When a flow is turbulent it is preferable to decompose the instantaneous variables (for example velocity components and pressure) into a mean value and a fluctuating value, i.e., 


$$
\begin{gathered}
\tilde{u}_{i}=U_{i}+u_{i} \\
\tilde{P}=P+p
\end{gathered}
$$

The continuity and the Navier-Stokes equations for an incompressible flow are given respectively as

$$
\begin{gathered}
\tilde{u}_{i, i}=0 \\
\rho\left(\tilde{u}_{i} \tilde{u}_{j}\right)_{, j}=-P_{, i}+\mu\left(\tilde{u}_{i, j}+\tilde{u}_{j, i}\right)_{, j}
\end{gathered}
$$

where ()$_{, j}$ denotes derivation with respect to $x_{j}$.

Inserting Eq. (3.17) into the above equations and averaging over time we obtain the time averaged continuity and Navier- Stokes equations as follows

$$
\begin{gathered}
U_{i, i}=0 \\
\rho\left(U_{i} U_{j}\right)_{, j}=-P_{, i}+\left[\mu\left(U_{i, j}+U_{j, i}\right)-\rho \overline{u_{i} u_{j}}\right]_{, j}
\end{gathered}
$$

A new term $\overline{u_{i} u_{j}}$ appears on the right-hand side of Eq. (3.21), which is called the Reynolds stress tensor. The tensor is symmetric (for example $\overline{u_{1} u_{2}}=\overline{u_{2} u_{1}}$ ). It represents correlations between fluctuating velocities. It is an additional stress term due to turbulence (fluctuating velocities) and it is unknown! Therefore, we need a model for $\overline{u_{i} u_{j}}$ to close the system of equations in Eq. (3.21). 
Closure Problem: This is called the closure problem: the number of unknowns (ten: three velocity components, pressure, six Reynolds stresses) is larger than the number of equations (four: the continuity equation and three components of the Navier- Stokes equations).

For steady, two-dimensional boundary-layer type of flow (i.e., boundary layers along a flat plate, channel flow, pipe flow, jet and wake flow, etc.) where

$$
V<U, \frac{\partial}{\partial x}<<\frac{\partial}{\partial y}
$$

where $x=x_{1}$ denotes streamwise coordinate, and $y=x_{2}$ coordinate normal to the flow. Equation (3.21), then reads

$$
\rho\left(\frac{\partial(U U)}{\partial x}+\frac{\partial \rho U V}{\partial y}\right)=-\frac{\partial P}{\partial x}+\frac{\partial}{\partial y}\left[\mu \frac{\partial U}{\partial y}-\rho \overline{u v}\right]
$$

Often the pressure gradient $\frac{\partial P}{\partial x}$ is taken to be zero.

Shear Stress: It should be noted that in addition to the viscous shear stress $\mu \frac{\partial U}{\partial y}$ on the right-hand side of Eq. (3.23) an extra turbulent term appears, the turbulent shear stress. The total shear stress is thus 


$$
\tau_{t o t}=\mu \frac{\partial U}{\partial y}-\rho \overline{u v}
$$

\subsubsection{The Reynolds Stress Model}

Beyond the Boussinesq approximation, there are several other approaches to model a turbulent flow. One of the most common of these models is called Reynolds Stress Model or simply the RSM model. The Reynolds stress model involves calculation of the individual Reynolds stresses, $\overline{u_{i} u_{j}}$, using differential transport equations. The individual Reynolds stresses are then used to obtain closure of the Reynolds-averaged momentum equation.

The exact form of the Reynolds stress transport equations may be derived by taking moments of the exact momentum equation. This is a process wherein the exact momentum equations are multiplied by a fluctuating property, the product then being Reynolds-averaged. Unfortunately, several of the terms in the exact equation are unknown and modeling assumptions are required in order to close the equations.

\subsubsection{The Large-Eddy Simulation or LES Model}

Turbulent flows are characterized by eddies with a wide range of length and time

scales. The largest eddies are typically comparable in size to the characteristic length of the mean flow. The smallest scales are responsible for the dissipation of turbulence kinetic energy. 
It is theoretically possible to directly resolve the entire spectrum of turbulent scales using an approach known as Direct Numerical Simulation or DNS. DNS is not, however, feasible for practical engineering problems. To understand the large computational cost of DNS, consider that the ratio of the large (energy-containing) scales to the small (energy-dissipating) scales is proportional to $\operatorname{Re}_{t}^{3 / 4}$, where $\operatorname{Re}_{t}$ is the turbulent Reynolds number. Therefore, to resolve all the scales, the mesh size in three dimensions will be proportional to $\operatorname{Re}_{t}^{9 / 4}$. Simple arithmetic shows that, for high Reynolds numbers, the mesh sizes required for DNS are prohibitive. Adding to the computational cost is the fact that the simulation will be a transient one with very small time steps, since the temporal resolution requirements are governed by the dissipating scales, rather than the mean flow or the energy-containing eddies.

The conventional approach to flow simulations employs the solution of the Reynoldsaveraged Navier-Stokes (RANS) equations. In the RANS approach, all the turbulent motions are modeled, resulting in a significant savings in computational effort. Conceptually, a large-eddy simulation (LES) is situated somewhere between DNS and the RANS approach. Basically large eddies are resolved directly in LES, while small eddies are modeled. The rationale behind LES can be summarized as follows:

- Momentum, mass, energy, and other passive scalars are transported mostly by large eddies.

- Large eddies are more problem-dependent. They are dictated by the geometries and boundary conditions of the flow involved. 
- Small eddies are less dependent on the geometry, tend to be more isotropic, and are consequently more universal.

- The chance of finding a universal model is much higher when only small eddies are modeled.

Solving only for the large eddies and modeling the smaller scales results in mesh resolution requirements that are much less restrictive than with Direct Numerical Simulation (DNS). Typically, mesh sizes can be at least one order of magnitude smaller than with DNS. Furthermore, the time step sizes will be proportional to the eddy-turnover time, which is much less restrictive than with DNS. In practical terms, however, extremely fine meshes are still required. It is only due to the explosive increases in computer hardware performance coupled with the availability of parallel processing that LES can even be considered as a possibility for engineering calculations.

Chapter 7 is about turbulent flow in static mixer. The specific turbulence models that are used in this research will be described in detail in Chapter 7. The accuracy, global performance and costs of the different turbulent models have been examined in order to select the most suitable and practical model in order to design/redesign and study mixer performance.

\subsection{Numerical Method}

The numerical simulation of the flow and mixing in a helical static mixer has been performed via a two-step procedure. In the first step, the flow velocity and the pressure is

computed. This has been achieved by the use of a commercial code. In order to apply the suitable boundary conditions to the computational domain and also in order to define 
some material properties (which are not constant), some modules were written and linked to the solver. The velocity values are then used as input to the second step that consists of the calculation of trajectories of particles (here, fluid elements) within the computed flow field. The velocity flow field and pressure drop across the mixer also are used in order to determine other parameters that are subject of interest in the performance study of a mixer. This has been achieved by the use of developed codes. These codes were programmed using FORTRAN 95 language; for the modules linked to the flow solver C programming language was used.

\subsubsection{Mesh Generation}

Numerical mesh generation is a crucial initial step of any computational simulation problem. Effective numerical processes for CFD problems depend on the geometric modeling and grid generation and grid quality. Hobbs and Muzzio [54] used an unstructured grid of 295,999 tetrahedral cells for a six-segment mixer. Mesh quality tests, if applied, were not mentioned. Based on past experience a tetrahedral mesh with no sliver cells for a geometry with highly twisted surface is difficult, if not impossible, to generate. The surface mesh shown in [54] includes surface cells which can lead to a three-dimensional mesh with sliver cells (which have four, nearly coplanar points). Also, there is no report on the study of the mesh density and its effects on the obtained results; however Byrde and Sawley [60] claim that the grid density needed to obtain realistic results are much more than the grid density used by Hobbs and Muzzio. Byrde and Sawley used four different mesh densities for a six-segment mixer: 122,880, 376,320, 
929,280, and 1,505,280 cells. They used an H-type mesh in the core of the pipe and an Otype mesh near the pipe wall [61]. The problem of using O-type mesh is an unnecessary concentration of small cells near the center of the pipe. Jones et al, [63] used a 1,035,125 cell structured mesh for a two-segment mixer with a long pipe and elbow prior to the mixing elements. The mesh type, the mesh generation algorithm, and the mesh density studies were not mentioned; however unnecessary small cells can be seen in the computational domain.

An alternative to a structured mesh, and the concomitant difficulties, is to use a high quality unstructured mesh [117]. Here, an unstructured hexagonal mesh (which compare to a tetrahedral mesh is much more suitable for this kind of problem involving shear flow) was generated to model the six-element static mixer inside a pipe; the geometry was modeled exactly, with no simplifications. To achieve the most accurate results of the solver all efforts have been taken to maximize the quality of the mesh. Instead of the traditional approach of three-dimensional unstructured mesh generation, in which the solid and boundary surfaces grid is generated prior to the volume meshing, here a novel technique is introduced. The flowfield geometry is cut by a large number of parallel planes perpendicular to the pipe centerline ( $x_{1}$-direction). These parallel constant- $x_{1}$ planes are distributed uniformly with a distance, say $\Delta s$, separated from each other (as illustrated in Fig. 3.2 for one twisted surface equal to the surface of one mixing element). The cross-section of each plane is meshed using an interval size equal to the separation distance between the planes, $\Delta s$. Figure 3.3 shows the cross-sectional mesh for two surfaces, which are two parts of an originally one cross-sectional circle cut by the twisted 
surface. The next step is to mesh the flowfield volume using these cross-sectional surface meshes. This technique leads to a high quality mesh, which, in turn, improves the rate of convergence of the flow solver. It also, makes the particle tracking calculations much faster and easier. Figure 3.4 shows two helical mixing elements and the resulting volume mesh on one mixing element.

Table 3.1 shows the outcome of different mesh quality tests performed on the generated mesh with 1,043,249 cells, used in this study for cases where $\operatorname{Re}=1,000$. Here, Maximum volume ratio means the ratio of the volume of the largest cell to the volume of the smallest one. Maximum face ratio is the ratio of the largest face to the smallest one. The Aspect ratio is the maximum value of the average lengths of the edges in three coordinate directions in a cell to the minimum value of the average lengths of the edges in three coordinate directions in that cell. The Diagonal ratio is the fraction of the longest diagonal to the shortest diagonal of a cell. The Edge ratio is the fraction of the longest edge to the shortest edge of a cell. The Mid-angle skewness is defined by the cosine of the minimum angle formed between the bisectors of the cell faces and is the maximum amount of the cosines of the three angles computed from the face-bisecting lines of the cell. Finally, equal-size skewness is a measure of skewness, which is defined as follows:

$$
\text { Equal-size skewness }=\left(V_{e q}-V\right) / V
$$

where $V$ is the volume of the cell, and $V_{e q}$ is the maximum volume of an equilateral cell the circumscribing radius of which is identical to that of the mesh element. More details 
about different computational mesh generation algorithms and different mesh quality tests can be found in $[117,118]$.

Considerations for Turbulent Flow: A successful turbulent flow computation requires a sufficiently fine grid particularly in regions of shear layers with a large rate of strain. For the problem studied here, a large portion of computational domain is in the near wall region. Depending on the near wall treatment used in a turbulent flow simulation, different meshing strategies must be used. For example if wall functions are used, an excessively fine mesh near the walls may increase the error, because the wall functions are not valid in the viscous sublayer. More details can be fo und in [97].

A standard approach to measure the fineness of the mesh in the near wall region is to monitor the distance of the computational cell centroid from the solid wall measured in terms of the viscous length, $y+=\frac{y \sqrt{\rho \tau_{w}}}{\mu}$. For the methods used in this study, a fine grid with $y+$ of the order of one is desirable in order to obtain accurate results. Table 3.2 gives the information related to the computational grid used in this study for the $k-\omega$ model used in this study. More information will be provided in Chapter 7.

A uniformly refined mesh leads to an enormous computational cost. Here, an adaptive mesh approach is used, which adds a grid node in the center of an existing computational cell and divides the cell into four new cells. The deviation of orthogonality of the original uniform grid used in this study is negligible and thus this approach does not degenerate the mesh. 


\subsubsection{Flow Field Solver}

The numerical simulation of the flow and mixing in the helical static mixer has been performed via a two-step procedure. In the first step, the flow velocity and the pressure are computed. The solver used in this study, for the flow computation part, is a commercial code: FLUENT (Fluent Inc., Lebanon, NH). The employed solver is a segregated, implicit, second order upwind [119], double precision, finite volume solver. Using the segregated approach, the governing equations are solved sequentially.

To obtain second-order accuracy, quantities at cell faces are computed using a multidimensional linear reconstruction approach $[119,120]$. Pressure-velocity coupling is achieved by using the SIMPLEC (SIMPLE-Consistent) algorithm [121]. The SIMPLEC pressure-velocity coupling scheme, part of the SIMPLE (Semi-Implicit Method for Pressure-Linked Equations) family of algorithms [122], is similar to the SIMPLE procedure, however it has been shown to accelerate the convergence in problems where pressure-velocity coupling is the main deterrent to obtaining a solution. As the convergence criterion, a scaled residual defined as the L2 norm [123] is employed. For, cases where there is no heat transfer between the fluid in the mixer and the solid surfaces, convergence criteria less than $10^{-5}$ is used. For the case of heat transfer, a convergence criterion is that the residual is less than $10^{-9}$ for temperature and is less than $10^{-6}$ for other variables.

Boundary Conditions: No-slip boundary conditions are applied to the solid surface of the static mixer and also at the wall of the pipe. A constant mass flow rate constraint is applied at the inlet and outlet boundaries. Different Reynolds numbers for the flow inside 
the pipe are obtained by varying the mass flow rate at the inlet boundary. The velocity profile for fully developed flow in a pipe (parabolic distribution for laminar flow and one seventh-root law for turbulent flow) is used at the flowfield inlet. The turbulence intensity is used to specify the turbulent boundary conditions at the flow inlet. The turbulence intensity is equal to $6.99 \%$ for the case of $\operatorname{Re}=3,000$, and is $6.56 \%$ for the case of $\operatorname{Re}=$ 5,000. Near solid walls an enhanced wall treatment, see Chapter 7, is employed for the cases for which wall treatment is needed.

For the heat transfer cases, the fluid temperature at the inlet is set to $298.15 \mathrm{~K}\left(25^{\circ} \mathrm{C}\right)$ for all cases but one, which is set to $278.15 \mathrm{~K}$. The mixer surface and the pipe surface in the entrance and exit regions where there are no mixing elements are adiabatic. The pipe surface temperature in the region including the static mixer is maintained at a constant for any run. Three constant values were used: $318.15 \mathrm{~K}, 348.15 \mathrm{~K}$, and $368.15 \mathrm{~K}$.

\subsubsection{Particle Tracking}

To determine the efficiency of a mixer, it is necessary to establish means by which the fluid mixing can be gauged both qualitatively and quantitatively. In the present study, this was achieved by calculating the trajectories of fluid particles in the flowfield of the mixer. This method avoids the problem of excessive numerical diffusion that is observed if the species continuity equations are solved [60]. For a steady flow, the particle trajectories correspond to streamlines. Therefore, trajectories are tracked by integrating the vector equation of motion, using the numerically computed velocity field as input. 


$$
\frac{d x_{i}}{d t}=u_{i}
$$

Some care must be taken whe $\mathrm{n}$ integrating the equation that describes particle motion in order to retain a sufficient degree of accuracy. Preliminary tests have indicated that while lower order schemes appear to provide acceptable results, they accentuate the problem of lost particles, that is, particle s that have trajectories cause them to be trapped near a solid wall, where the local velocity is zero, or bave the computational domain [60]. For the cases presented, a fourth order Runge-Kutta integration algorithm with adaptive time-step-size control was employed. In addition, to avoid problems near stagnation points, the numerical integration of the streamline equation was performed using a fixed spatial increment rather than a fixed time step. By this method, the trajectory of each fluid particle released at flowfield inlet can be tracked and the particle locations within different cross-sections of the flowfield, e.g., at the end of each mixing element, can be obtained. Figure 3.5 shows the trajectory of one particle through the mixer.

Furthermore, it was found that the mean velocity has an impact on the number of lost particles. The mass flow rate can be written as the following

$$
\dot{m}=\frac{\pi d \mu}{4} \operatorname{Re}
$$


For a given geometry, the mass flow rate is only a function of viscosity for any specified Reynolds number. Therefore, by changing the values of the working fluid viscosity, different mean velocities can be obtained for the same Reynolds number. For the case of $\operatorname{Re}=5,000$, using the $k-\omega$ turbulence model, it was found that by decreasing the magnitude of order of the mean velocity by four, the number of lost particles decreased by a factor of 0.7158 . It is worthwhile mentioning that this effect is only significant for high Reynolds numbers.

To obtain an accurate evaluation of the mixing, a study of the trajectories of a large number of particles has been undertaken. At the entry of the inlet pipe section, 501,740 zero-volume and zero-mass particles were distributed uniformly over the half circular plane of the inlet surface. The diameter of this plane is perpendicular to the front edge of the first mixing element in the pipe. This is a simplified model for the diametrical feeding of the mixer with two components fluids. Particle trajectories corresponding to only one of the working fluids are calculated.

The computational grid consists of constant- $x_{1}$ planes, which can be used as buckets. These buckets are used to find the flow velocity in each point of flowfield. This search is fast and efficient and increases the speed of the integration code of particle tracking. While integrating, the time step is chosen based on the axial velocity $\left(u_{1}\right)$ so that the particle currently existing on one constant- $x_{1}$ plane falls on the next constant- $x_{1}$ plane. In the next step, a search is performed in this new constant- $x_{1}$ plane to find the nearest neighboring grid points surrounding the particle. Once these neighboring grid nodes are located, an inverse distance weighted average function can be constructed to calculate the 
velocity components for the particle. Although for the case of turbulent flow regime the computational grid is not uniform, it is still possible to use the method mentioned here to track the trajectories of particles by the use of a tree data structure. 


\begin{tabular}{|l|c|}
\hline Maximum volume ratio & 1.000058 \\
\hline Maximum face ratio & 1.008443 \\
\hline Aspect ratio & $1.0083895 \sim 1.008390$ \\
\hline Diagonal ratio & $0.999999 \sim 1.000001$ \\
\hline Edge ratio & $1.008389 \sim 1.009009$ \\
\hline Mid-angle skewness & $<0.000001$ \\
\hline Equal-size skewness & $<0.000001$ \\
\hline
\end{tabular}

Table 3.1: Mesh quality tests result 


\begin{tabular}{|c|c|c|c|c|}
\hline $\mathrm{Re}$ & Number of cells & Model & Minimum $y+$ & Maximum $y+$ \\
\hline 3,000 & $2,335,690$ & $k-\omega$ & 0.11 & 2.96 \\
\hline 5,000 & $2,822,681$ & $k-\omega$ & 0.17 & 4.35 \\
\hline
\end{tabular}

Table 3.2: Mesh information for the turbulent flow cases using the $k-\omega$ model 


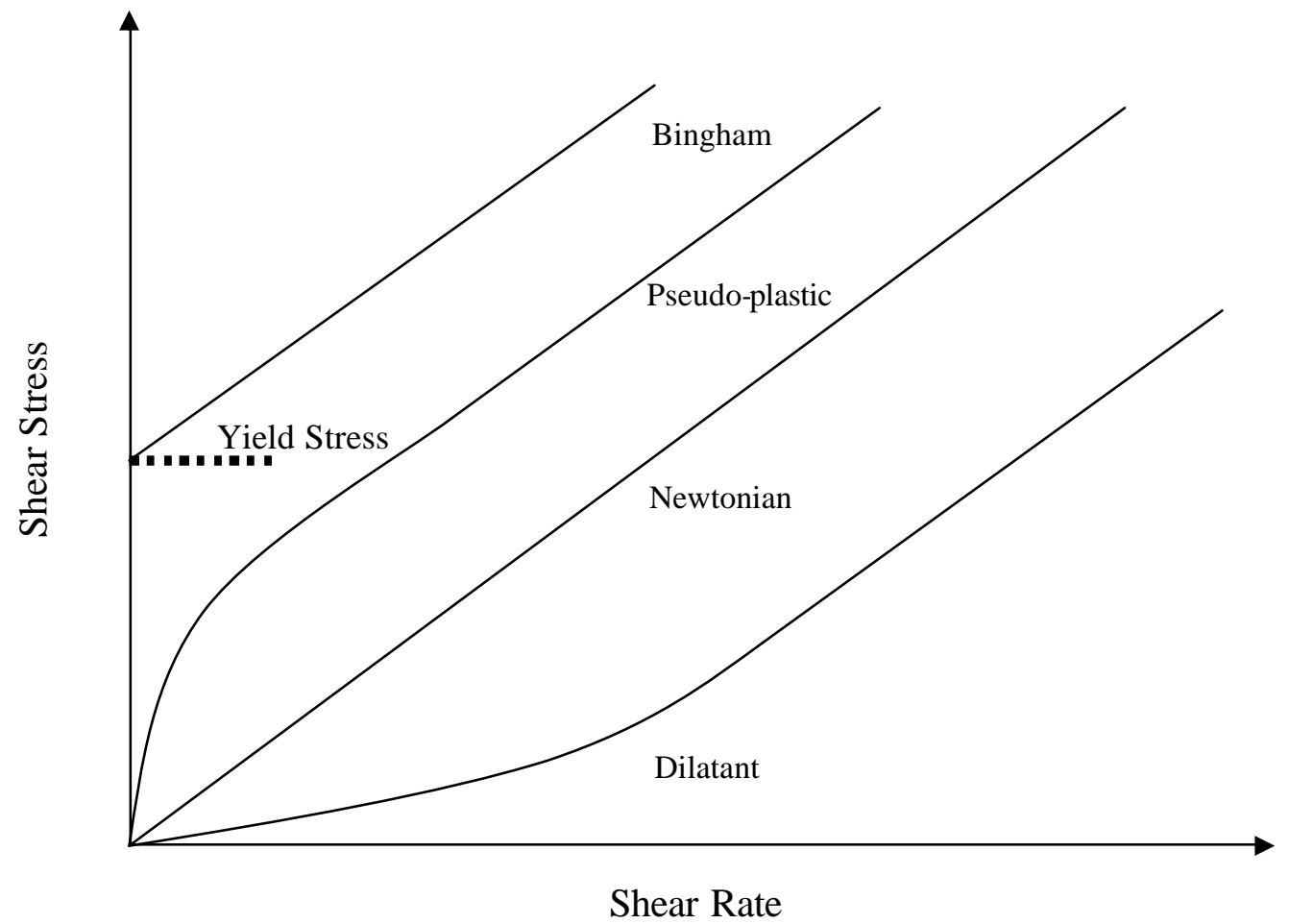

Figure 3.1: Flow curves for Newtonian and time-independent non-Newtonian Fluids 


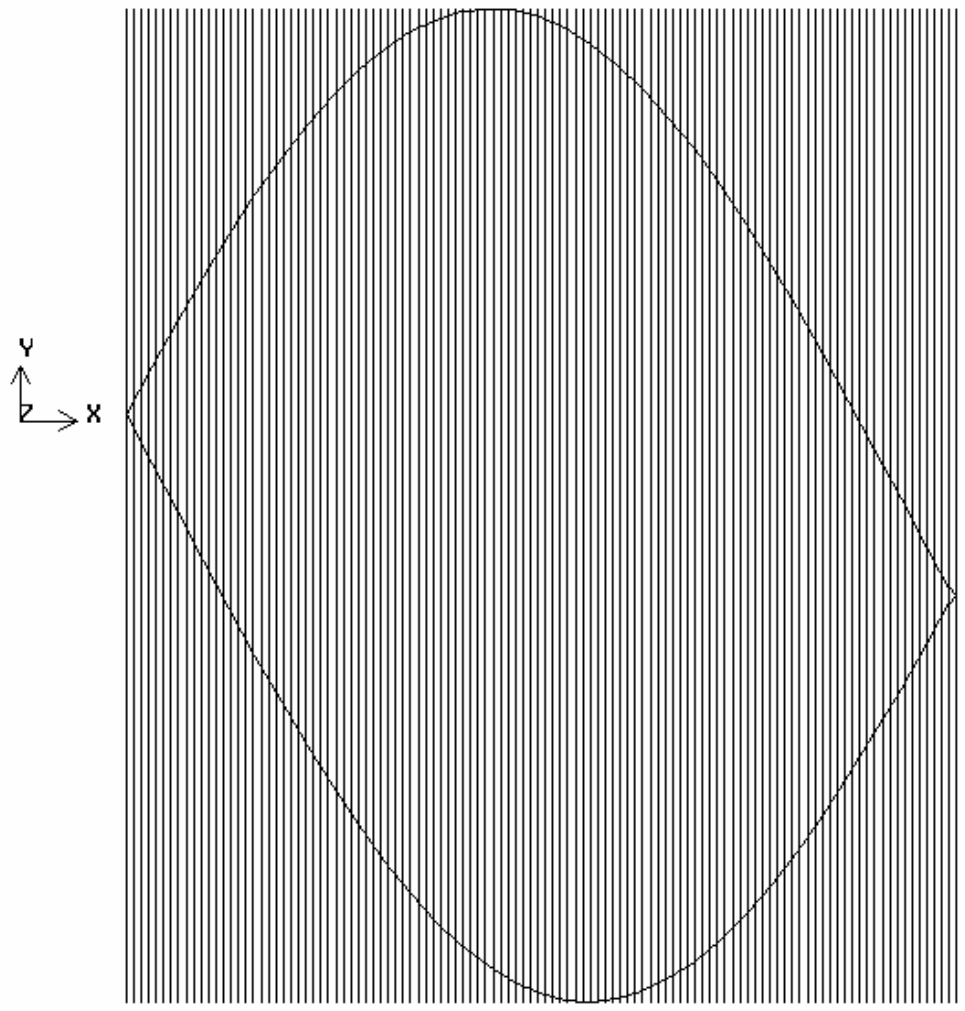

Figure 3.2: Computational domain across one helical surface is cut by a large number of parallel planes perpendicular to the flowfield centerline 


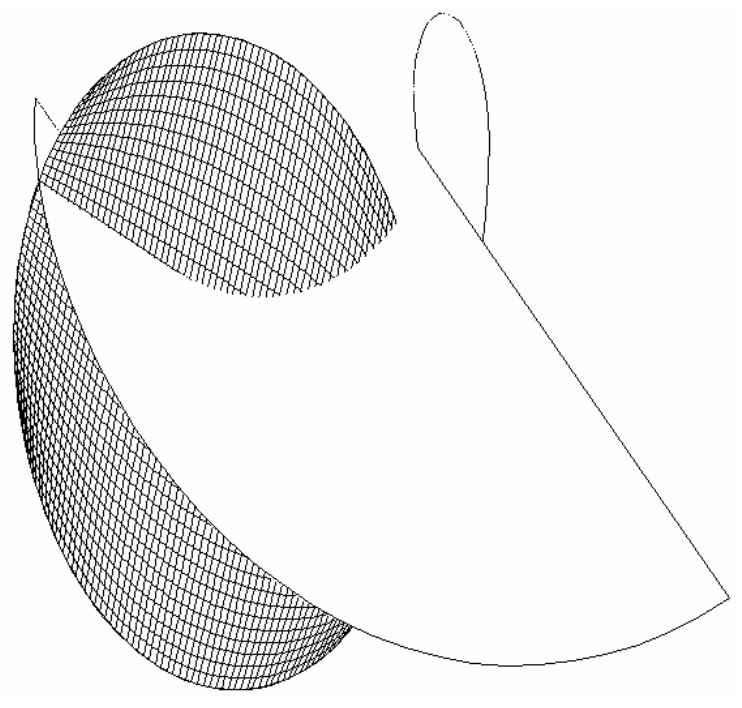

Figure 3.3: Cross-sectional mesh across one helical surface 


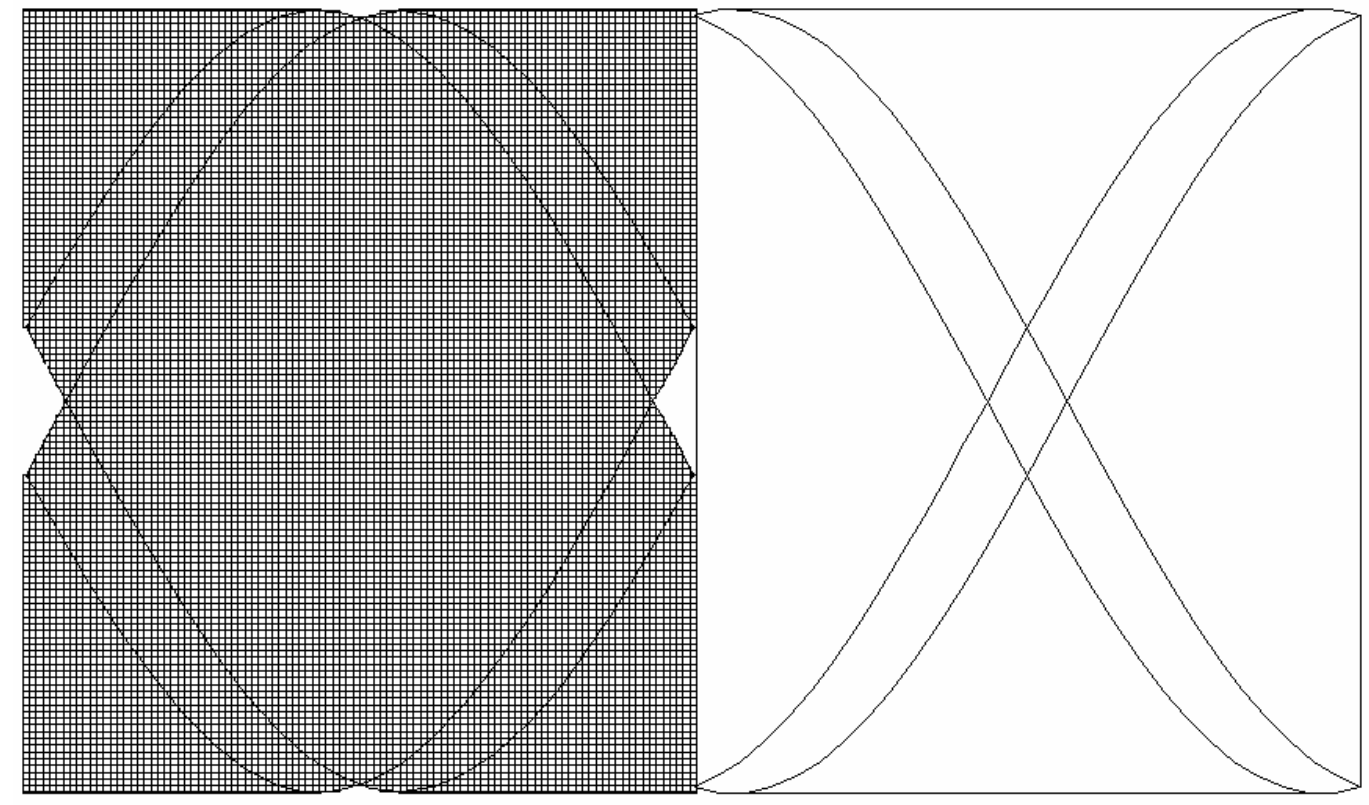

Figure 3.4: Volume mesh for one mixing element 


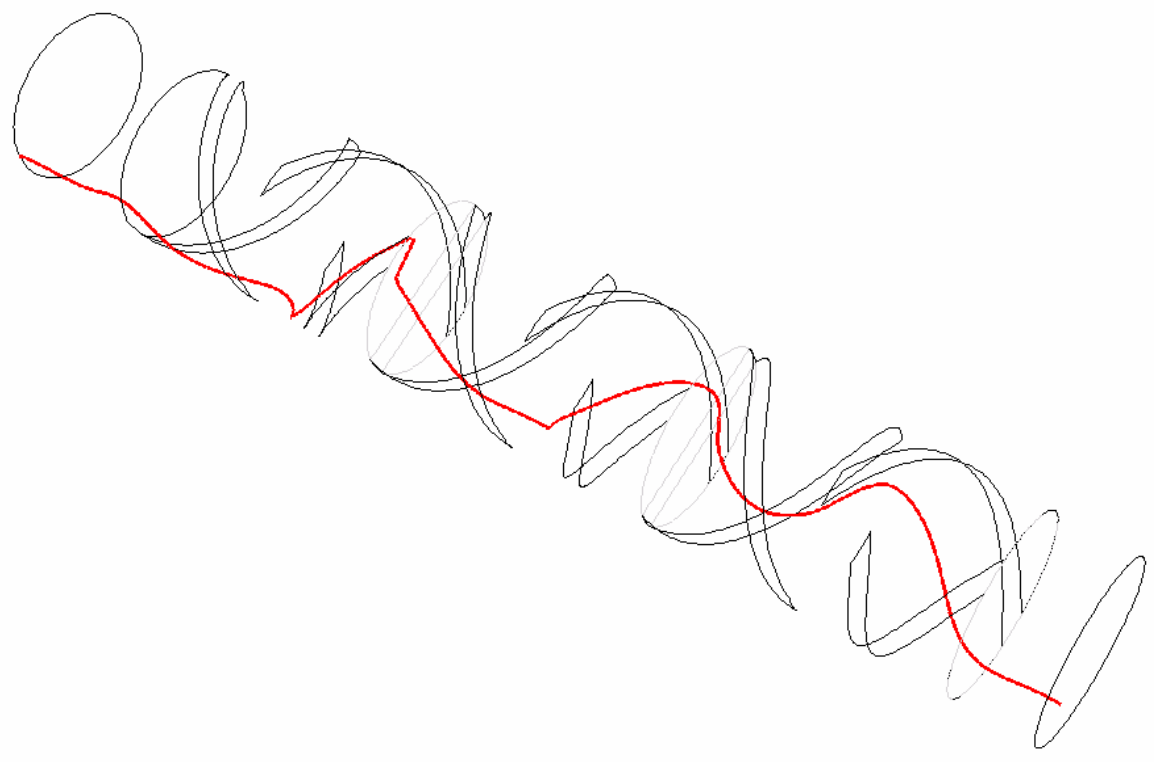

Figure 3.5: Trajectory of one particle through the mixer 


\section{CHAPTER FOUR}

\section{STATIC MIXER PERFORMANCE MEASUREMENT}

There are several parameters that are used in order to measure the performance of a static mixer. Some of these parameters are based on experimental data. Some other

parameters can be measured experimentally or they are determined using results of a numerical simulation. In this chapter, parameters that are used in this research to study the performance of an industrial helical static mixer are described. A new parameter is introduced, which has advantages over parameters that are currently used. For example, it is a global parameter and also it is easily determined.

\subsection{Pressure Drop}

The pressure drop across a static mixer has been experimentally measured for Newtonian and non-Newtonian fluids, and for laminar and turbulent flows. The measurements were made since pressure drop is essential in order to correctly size the extruder or pump, feeding the static mixer. Pressure drop across the mixer indicates the 
energy required to maintain the through flow. It also can be used to determine the static mixer efficiency.

\subsection{Temperature Difference Ratio}

To decrease the temperature gradient of two fluids entering into a pipe, helical static mixers can be employed. Effectiveness of static mixers can be estimated using a nondimensional ratio of the temperature difference in fluid at a specific flow cross-section in a pipe containing a static mixer to that in an empty pipe of the same diameter. If this ratio is greater than one, the static mixer is not effective; however this situation does not actually occur in practice. The smaller this ratio is, the more efficient the static mixer becomes. When this ratio is zero at a flow cross-section, it means that in there is no temperature difference in that cross-section of the pipe which contains a static mixer. When this ratio is one, it means that static mixer has no impact on temperature blending

of fluid elements in the flow. Temperature Difference Ratio (TDR), in a given flow crosssection, can be defined as

$$
\mathrm{TDR}=1-\frac{\left(T_{\max }-T_{\min }\right)_{\text {pipe with mixer }}}{\left(T_{\max }-T_{\text {min }}\right)_{\text {pipe without mixer }}}
$$

TDR is between zero and one. The higher TDR, the more efficient the static mixer is. 
Consider a case that TDR is equal to one. For such a case, there is no temperature gradient in the fluid inside the pipe that contains a static mixer. It is possible that the temperature gradient of fluid inside an empty pipe of the same diameter is also very small, or the temperature gradient might be significant. Therefore, when TDR is equal to one, it cannot provide information regarding the performance of static mixer. In this situation, other parameters rather than TDR should be considered in order to evaluate the performance of mixer.

\subsection{Heat Flux Ratio}

The ratio of heat flux from the pipe wall into the fluid in a pipe containing a static mixer to the heat flux in a pipe with no mixer is the heat flux ratio. In practice, helical static mixers, which are used in heat exchangers, are made of conductive materials such as cupper. Therefore, static mixers are able to increase the heat flux significantly. For cases in which the static mixer is made from a non-conductive material, the heat flux ratio can be close to one or slightly less than one, depending on the flow conditions.

\subsection{Residence Time Distribution}

The Residence Time Distribution (RTD) is used to characterize the uniformity of the history of fluid elements in a static mixer. A similar history for all fluid elements in the flow is a desirable feature in order to provide the uniformity of the product quality. This 
can be achieved by a narrow distribution of the residence times for chemical reactors. RTD for flows in a static mixer has been studied experimentally [46] and numerically $[19,20,124]$. In this thesis, the RTD for a flow in a helical static mixer was calculated by tracking a large number of uniformly spaced, zero-mass, zero-volume, particles initially located in the top half of the flow field inlet. The trajectories of these particles were tracked through the flow via integration of the pathline motion equation:

$$
\frac{d x_{i}}{d t}=u_{i}
$$

The residence time of each particle was measured from the point when the particle passes the cross-sectional plane of the leading edge of the first mixing element to the point when the particle passes the cross-sectional plane of trailing edge of the last mixing element. The measured residence time is non-dimensionalized by the residence time of a fluid particle traveling at the bulk flow velocity in a pipe with no mixer. Given the value of the non-dimensionalized residence time $\left(t^{*}\right)$ for all fluid particles which have passed the cross-sectional plane, the fraction of the volumetric flow, which has a residence time between $t^{*}$ and $t^{*}+d t^{*}$ can be calculated. This parameter is known as the distribution function, $f\left(t^{*}\right)$. 


\subsection{G-Value}

The purpose of this section is to describe calculation of the mean $G$-value for a helical static mixer. About sixty years ago, Camp and Stein [125] developed the root-meansquared G-value to characterize mixing in flocculation basins by analogy with the shear rate in a simple, one-dimensional, laminar shear flow: Couette flow. The theoretical development was an extension of the role of the velocity gradient in orthokinetic flocculation in pure shear flows. Essentially, Camp and Stein derived the G-value to represent the equivalent velocity gradient for a three-dimensional, turbulent flow. The Gvalue has been extended to characterize a variety of flowfield-induced mixing processes including the rapid mixing of coagulants, floc breakup, hydraulic flocculation, air and water induced-circulation in channels, short-circuiting in tanks, and filter cleaning during backwash [126]. Numerous authors [126-133] have discussed the inadequacies of the Gvalue.

The G-value reduces the evaluation of the process of mixing in extremely complex flowfields to a single number, a reduction that is unlikely to be successful regardless of the theoretical assumptions. Consequently, the G-value became a universal measure of mixing in the following decades.

However, it has been demonstrated that the original derivation of the G-value was flawed for three-dimensional flows [126,134] and cannot be universally applied to different types of mixers or different size mixers. Nonetheless, the G-value remains entrenched in the engineering literature and continues to be used [63]. Because of that and also because it can be calculated easily, it is not entirely futile to use the obtained 
numerical results to calculate the G-value and explore it. For any in-line mixer, the Gvalue is calculated based on the energy losses that occur in the mixer as follows:

$$
\mathrm{G} \text { - value }=\left(\frac{Q \Delta p}{\mu V_{m}}\right)^{1 / 2}
$$

For the static mixer studied here Eq. (4.3) can be written as:

$$
\mathrm{G} \text { - value }=\frac{1}{2}\left(\frac{\pi D}{V_{m}}\right)^{1 / 2}\left(\frac{\operatorname{Re} \Delta p}{\rho}\right)^{1 / 2}
$$

As can be seen from Eq. (4.4), for a given geometry and a specific Reynolds number, the G-value is a function of material density and the pressure drop of the flow in the mixer.

\subsection{Structure Radius}

As a quantitative measure of the fluid mixing, the size of flow structures at different flow cross-sections is considered. The two-dimensional structure radius at a given axial location, $r_{s}$, normalized to the pipe radius, has been defined to correspond to the radius of the largest circle that can be drawn around a particle of one of the fluid components that does not contain any particles of the other fluid component [49]. This structure radius corresponds to the striation thickness generally measured experimentally [61]. 
A computational grid needs to be significantly fine in order to produce mesh independent values for the structure radius. Byrde and Sawley studied pressure drop and structure radius for flow across a helical static mixer, when $\mathrm{Re}=100$. When they increased the number of computational cells from 122,880 to $1,505,280$ the value of predicted normalized pressure drop increased less than 3\%, but the predicted radius structure decreased more than $35 \%$. And even using 1,505,280 cells, the result is not still mesh independent [61].

The number of particles that are released in the flow inlet has a major impact on the predicted structure radius. Moreover, the fact that these particles have no dimension (they are mass-less and zero-volume particles) makes the determination of structure radius, based on location of these particles, somewhat problematic.

\subsection{Particles Distribution Uniformity}

The G-value is a parameter which is easy to calculate using the results of a numerical simulation; however it is not applicable to different types of mixers or different size mixers. On the other hand, the structure radius has a precise definition, but it is not easy to calculate the structure radius using the numerical results. Here, a new parameter is introduced that can be universally applied to different types of mixers or different size mixers and its determination from the numerical results is straightforward.

The perfect mixing of two fluids can be defined as a uniform distribution of all fluid particles in the flowfield cross-section. Based on this concept, assume that the cross- 
section of the flow is divided into very small, identical in shape and size, plane sectors. If $N_{s}$ is the total number of plane sectors, and $N_{(i)}$ is the number of particles placed in the ith sector, then the particle distribution function of the first kind for the ith sector is given by:

$$
p d_{1}(i)=\frac{\left|\frac{N_{p}}{N_{s}}-N_{(i)}\right|}{N_{p}}
$$

Now assume the flowfield cross-section is divided into equal angles by a large number, say $N_{l}$, of symmetry lines. If the number of particles on the left side of the ith symmetry line is $N_{\text {Left }}(i)$, and the number of particles on the right side of this line is $N_{\text {Right }}(i)$, then the particle distribution function of the second kind for the ith symmetry line is given by:

$$
p d_{2}(i)=\frac{\left|N_{\text {Left }}(i)-N_{\text {Right }}(i)\right|}{N_{p}}
$$

Using the particle distribution functions of the first and the second kind, the Particles Distribution Uniformity (PDU) can be defined as: 


$$
\mathrm{PDU}=\left(1-\frac{1}{2\left(1-\frac{1}{N_{s}}\right)} \sum_{i=1}^{N_{s} \rightarrow \infty} p d_{1}(i)\right)\left(1-\frac{1}{N_{l}} \sum_{i=1}^{N_{l} \rightarrow \infty} p d_{2}(i)\right)
$$

This function varies between zero (a completely non- uniform distribution of particles) and one (a totally uniform distribution of particles). It is worthwhile noticing that to obtain a meaningful value for PDU, $N_{s}$ and should be smaller or equal to $N_{p}$ and $N_{l}$ should be smaller or equal to $1 / 2 N_{p}$; also each of the $N_{s}$ and $N_{l}$ should be greater than or equal to 2 .

The PDU value can be easily obtained using Eq. (4.7), whenever it is possible to divide the flow cross-section into identical shaped and sized plane sectors. However, there are geometries which may not be divided into identical sectors. For such cases a modification to Eq. (4.7) is needed. In fact the particle distribution function of the first kind, $p d_{1}$, can be defined in a more general way, which also makes the number of calculations needed to determine it less.

Assume that the cross-section of the flow is divided into very small, similarly shaped strips, which have equal areas. Figure 4.1 shows a cross-section which is divided to several similar strips. In the center of the cross-section instead of a strip, there a region with the same shape of cross-section; when the number of these strips increases the area of this region goes to zero. In such a case, the thickness of these strips also goes to zero. In this circumstance, the length of each strip is proportional to the number of particles that lie on that strip if all the particles are distributed uniformly in the cross-section. It is 
possible to allocate a radius to each strip, $r_{i}$, which is a characteristic length showing the distance of the strip from the center of cross-section. For a circular cross-section, each strip is a circle and its distance from the center of cross-section is its radius. If the total number of strips (radial portions) is $N_{r}$, then the radius of the ith strip is:

$$
r_{i}=\sqrt{\frac{i}{N_{r}}} R
$$

where $R$ is the characteristic length of cross-section. For a circular cross-section, $R$ is simply its radius. If we defined $S_{i}$ as:

$$
S_{i}=\sum_{i=1}^{N_{r} \rightarrow \infty} \sqrt{i}
$$

In perfect mixing conditions, the number of particles on the ith strip is $\frac{\sqrt{i}}{S_{i}} N_{p}$, which $N_{p}$ is the total number of particles. Now if $N_{(i)}$ is the number of particles placed on the ith strip, in a general condition, then the modified particle distribution function of the first kind for the ith strip is given by:

$$
p d_{1}(i)=\frac{\left|\frac{N_{p}}{S_{i}} \sqrt{i}-N_{(i)}\right|}{N_{p}}
$$


The PDU value can be easily obtained by substituting Eq. (4.10) into Eq. (4.7). It should be noted that both Eqs. (4.5) and (4.10) give the same value for $p d_{1}$, when both approaches are possible.

\subsection{Static Mixer Efficiency}

To determine the mixer efficiency, a combination of pressure drop across the static mixer and the structure radius can be used. Byrde and Sawley defined the mixing efficiency as:

$$
e f f=\frac{1}{\Delta p r_{s}}
$$

where $\Delta p$ is the total pressure drop along the static mixer and $r_{s}$ is the structure radius at the end of the last mixing element [61]. The efficiency defined by Byrde and Sawley is not dimensionless.

Similarly, the mixer efficiency can be defined by using the PDU value as:

$$
e f f=\frac{P D U}{\Delta \hat{P}}
$$


In this thesis, Eq. (4.12) is used to determine helical static mixer efficiency. $\Delta \hat{P}$ is nondimensional pressure and is defined as:

$$
\Delta \hat{P}=\frac{\text { Pressure drop in a pipe with static mixer }}{\text { Pressure drop in a pipe without static mixer }}
$$

The PDU value at the end of the last mixing element is used in Eq. (4.12). 


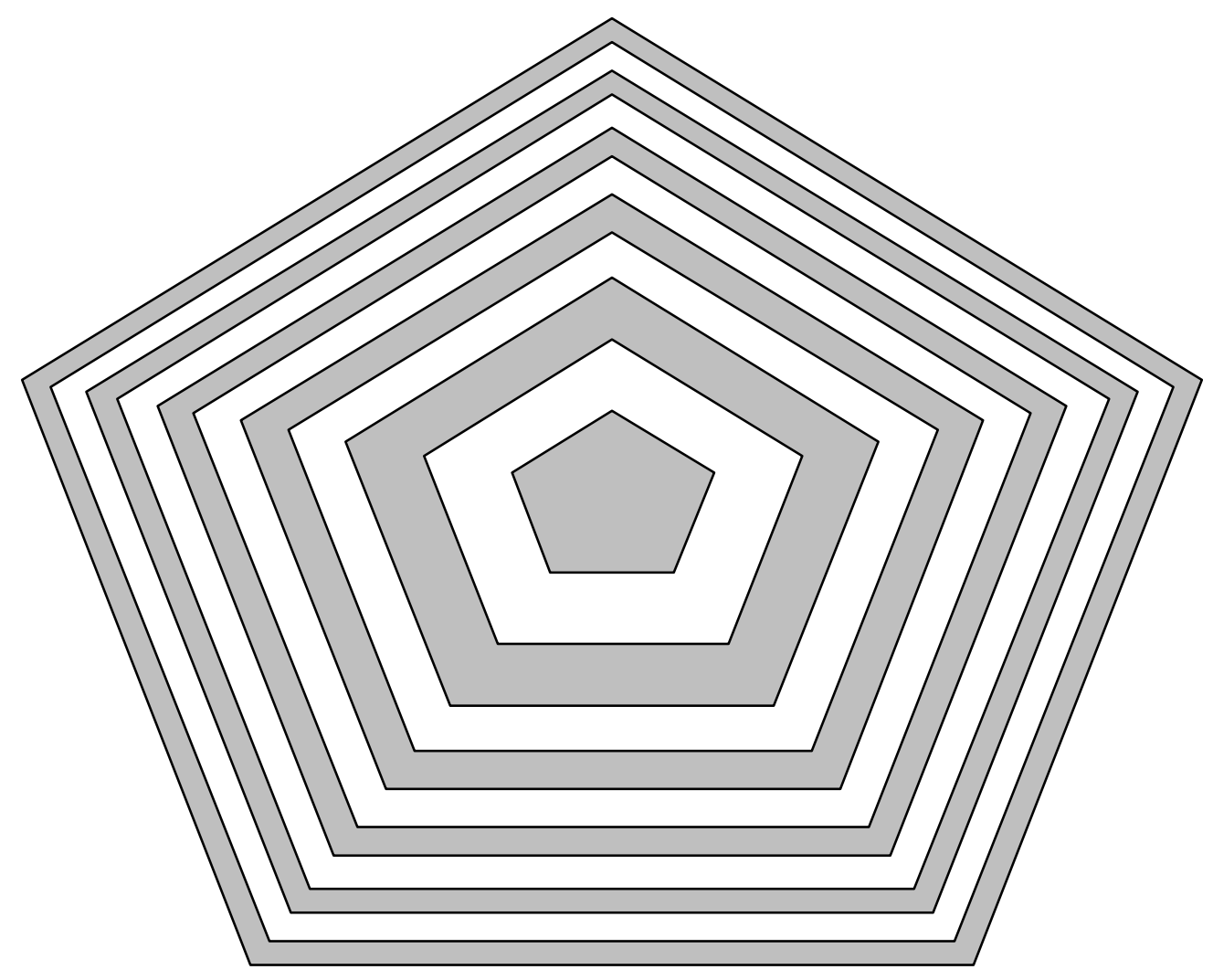

Figure 4.1: A flow cross-section divided by similar strips with equal area 


\section{CHAPTER FIVE}

\section{CREEPING AND LAMINAR FLOWS IN HELICAL STATIC MIXER}

Helical static mixers are widely used in continuous mixing, heat transfer processes and chemical reactions of high viscous fluids in low Reynolds number flows. In this chapter, the performance of a helical static mixer in creeping and laminar flow conditions is studied.

\subsection{Numerical Solution Accuracy}

It is important that the accuracy of the numerical solutions be analyzed before confidence in the predictive ability of the numerical techniques can be justified. For the present study of mixing under non-creeping flow conditions, there is unfortunately an absence of experimental data sufficiently detailed and accurate to undertake a complete validation of the numerical results. Comparison with certain experimental values of the pressure drop across the mixer, measured for the standard element twist angle, shows very good agreement over a range of flow conditions; however, good agreement with the experimental data for such a global measure is not sufficient to guaranty accurate simulation of the flow in the complex geometry. Therefore, a detailed mesh convergence 
study was indicated in order to determine that the computational mesh employed in the present study is sufficiently refined to provide good numerical resolution. A coarse mesh is not able to resolve adequately the complex flow behavior and leads to a considerably large number of lost particles. Moreover, a study conducted using different numbers of particles for the mixing analysis, has shown that the results are independent of the number of particles. The use of an inadequate number of particles to analyze mixing can be misleading and create a false impression of good mixing in the presence of the fine flow structure [60].

As a quantitative measure of fluid mixing, the size of the flow structures at the flowfield outlet is considered. The two-dimensional structure radius at a given axial location, $r_{s}$, normalized to the pipe radius, has been measured using different mesh sizes in order to study the computational grid convergence.

\subsubsection{Solution Accuracy Evaluation}

For any numerical simulation, error estimation is an important and essential task. The validity of the obtained results can be examined via various numerical studies or by comparison with experimental data. In the present study the only available experimental data is the pressure drop inside the pipe that includes a helical static mixer. Here, the pressure drop is defined as the absolute difference between the area-weighted average pressure at the computational flowfield upstream and the area-weighted average pressure at the flowfield downstream. This is an important quantity for a static mixer and provides a measure of the required energy for using the mixer in the pipeline. The range of the 
flow Reynolds number studied in this chapter range from 0.01 (creeping flow) to 1,000 (laminar flow). The maximum difference between the measured value of the pressure drop and the computed pressure drop is for the case of $\operatorname{Re}=100$, which is $2.85 \%$.

Mesh Convergence: The effects of the size of the mesh used for the flowfield computations and the influence of the number of particle trajectories calculated to analyze the mixing process have been investigated.

Different mesh sizes, that contain 446,489 cells, 613,668 cells, 1,043,249 cells, and $2,210,186$ cells, were used to numerically determine the flowfield. The obtained velocity field in each case was used to calculate the trajectories of 501,740 particles. Figures 5.1a and $5.1 \mathrm{~b}$ present the structure radius values for each of these meshes for $\operatorname{Re}=10$ and $\operatorname{Re}$ $=100$, respectively. Figures 5.2a and 5.2b present the computed pressure drops for each of these computational grids for $\operatorname{Re}=10$ and $\operatorname{Re}=100$, respectively. As the computational mesh is made finer, the results for both the structure radius and the pressure drop values show convergence. For the case of $\operatorname{Re}=100$, the difference in the computed pressure drop for the coarser mesh with 613,668 cells and the finer mesh with $1,043,249$ cells is $7.16 \%$; however the difference between the obtained structure radiuses for these two meshes is $21.43 \%$. Therefore, the general behavior of the flow is not as dependent on the mesh density as the mixing process is. However, for a mesh with $2,210,186$ cells, the variation of obtained results is negligible compared to the results obtained using a mesh with 1,043,249 cells. Also, for the case of $\operatorname{Re}=10$, the difference in the computed pressure drop for the coarser mesh with 613,668 cells and the finer mesh with $1,043,249$ cells is $8.26 \%$, and the difference between the obtained structure radius is 38.46\%. This is in agreement with the results obtained by Byrde and Sawley [61]. 
Moreover, the mesh size has a significant impact on the number of lost particles. The number of lost particles is reduced by using a finer mesh, e.g., for the case of $\operatorname{Re}=10$, the number of lost particles for the finer mesh (with 1,043,249 cells) is $90.06 \%$ of the number of lost particles for the coarser mesh (with 613,668 cells).

Number of Particles: Different numbers of particles $\left(N_{p}=457,1,208,7,728,31,180\right.$, $195,784,501,740,784,272$, and $1,225,784$ ) were used to analyze the mixing. By increasing the number of particles released at the flowfield inlet, it is possible to discern finer structures and thus quantify the mixing efficiency with a higher precision. Figure 5.3 shows the influence of the number of particles released at the flowfield inlet from the reference point of a stationary observer. A relatively small $N_{p}$ gives the impression that a high level of mixing has been achieved at the exit of the mixer. As the $N_{p}$ is increased, the presence of the striations associated with the mixing process is more and more evident. It can be seen that 501,740 particles at the flow inlet can give a correct image of mixing; although using a higher value for $N_{p}$ leads to a more pronounced mixing pattern, but the difference between the obtained results is not that significant to justify the long CPU time needed for calculations.

\subsection{Numerical Predictions}

Using the numerical methods described in Chapter 3, the flow in a six-element static mixer has been analyzed for a number of different flow conditions. In each case the initial 
conditions are set so that the axial velocity $\left(u_{1}\right)$ is equal to bulk velocity and $u_{2}=u_{3}=0$. Figure 5.4 shows the residual history for three different Reynolds numbers. The number of iterations required to obtain a converged solution was found to be a function of the flow regime. For a mesh containing 1,043,249 cells, 350 iterations for creeping flows, Re $=0.01$, were necessary to reach converged results. One the other hand, for $\operatorname{Re}=1,000$, 610 iterations were needed for convergence. A scaled residual less than $10^{-5}$ for all scalar equations is used here, as the convergence criterion.

\subsubsection{Flowfield Parameters}

Using water properties $\left(\rho=998.2 \mathrm{~kg} / \mathrm{m}^{3}\right.$ and $\left.\mu=0.001003 \mathrm{~kg} / \mathrm{m} . \mathrm{s}\right)$, the velocity field was obtained for $\operatorname{Re}=1, \operatorname{Re}=10, \operatorname{Re}=100$, and $\operatorname{Re}=1,000$. The cross-sectional velocity fields are illustrated in Figs. 5.5-5.8, which show the cross-sectional projection

of the velocity vectors at the end of the $2^{\text {nd }}$, the $4^{\text {th }}$, and the $6^{\text {th }}$ elements, respectively from top to bottom, and correspond to $\operatorname{Re}=1, \operatorname{Re}=10, \operatorname{Re}=100$, and $\operatorname{Re}=1,000$. To obtain clear images, the length scale of the product of the velocity vector and the Reynolds number in each case is set to a constant. The cross-sectional velocity fields for $\operatorname{Re}=1, \operatorname{Re}=0.1$, and $\operatorname{Re}=0.01$ (not shown in here) are almost identical. Different flow patterns for creeping and laminar flows can be seen. Also, it is observed that after the second element, four velocity zones are created. These zones combine together and create two velocity zones, as the flow goes through the mixer. The transition from four zones to two zones occurs sooner, when the Reynolds number is increased. For higher Reynolds number flows, regions of strong cross-sectional vortices are distinguishable. 
Values of mean axial velocities and mean velocity magnitudes at different flow crosssections are shown in Table 5.1. As expected from the conservation of mass, the averaged axial velocity is proportional to Reynolds number and it is the same for each case at the end of the $2^{\text {nd }}$ and the $4^{\text {th }}$ mixing elements. At the end of the last element, the averaged axial velocity is decreased as the flow cross-section is increased. As can be seen, the ratio of the averaged axial velocity to the averaged velocity magnitude is about 0.9 for $\mathrm{Re}=1$ and $\operatorname{Re}=10$ at all flow cross-sections, while it is about 0.7 for $\operatorname{Re}=100$, and it is about 0.5 for $\operatorname{Re}=1,000$. Therefore, the cross-sectional component of the velocity vectors are increased as the Reynolds number increases, which can lead to a higher degree of mixing.

Figures 5.9-5.12 show vorticity contours at different flow cross-sections for $\operatorname{Re}=1$, $\operatorname{Re}=10, \operatorname{Re}=100$, and $\operatorname{Re}=1000$, respectively. The mean and the maximum vorticity magnitudes at the end of each mixing element are presented in Table 5.2. As may be expected the mean and the maximum vorticity magnitudes are increased by increasing the Reynolds number. Therefore, a higher degree of mixing is expected in the same flow cross-section when the Reynolds number is high.

As the flow goes through the mixer the mean and the maximum vorticity magnitudes change. These values increase from the first mixing element to the second element; however, they decrease from the second to the third element. The mean and the maximum vorticity magnitudes increase for lower Reynolds numbers and they decrease for higher Reynolds numbers, when the flow passes the fourth mixing element. These values increase for all flow Reynolds numbers from the fourth mixing element to the fifth element. Also, it is observed that the vorticity magnitude is smaller overall at the end of the last mixing element compared to the vorticity magnitude at the other elements; the 
flow after the last mixing element gradually becomes similar to the flow in a pipe with no mixer. For the first five mixing elements the mean vorticity magnitude is about $20 \%-25 \%$ of the maximum vorticity magnitude. For the last mixing element, the mean vorticity magnitude is much smaller than the maximum vorticity magnitude. This suggests that flow around the internal mixing elements has a higher mixing potential; although by increasing the Reynolds number the contrast between the mean and the maximum values of vorticity at the last mixing element and other mixing elements is less pronounced.

Table 5.3 presents the ratio of flow pressure drop across the six-element static mixer to the flow pressure drop in an empty tube. The pressure drop is based on measuring the static pressure between two flow cross-sections located at a distance of half an element length from the leading edge of the first element and trailing edge of the sixth element. The pressure drop ratio increases dramatically by increasing the Reynolds number, suggesting that this kind of mixer is more suitable for low Reynolds number flows. Wilkinson and Cliff [33] proposed the following correlation between Reynolds number and pressure drop ratio for Kenics mixers:

$$
\text { Pressure drop ratio }=7.19+0.03125 \mathrm{Re}
$$

The pressure drop ratio in the helical static mixer studied here shows almost the same pattern of behavior as a function of Reynolds number; however the pressure drop ratio values are approximately two times higher than those of the Kenics mixers. This can be 
explained by the fact that the helical static mixer studied in this dissertation is more compact, resulting in a larger pressure drops.

\subsubsection{Particle Trajectories}

The flow field velocity predicted by the flow solver and applied boundary conditions is used to determine the redistribution of zero-mass and zero-volume elements, in each flow cross-section. These elements were initially uniformly distributed over the half of the inlet flow field. Trajectories of particles injected into the mixer flow inlet have been calculated. Figures 5.13 and 5.14 show the redistribution of the particles at the end of each mixing element for $\operatorname{Re}=1$ and $\operatorname{Re}=100$, respectively. These plots illustrate the redistribution of the released particles via the combined effects of flow division and reversal, resulting in stretching and folding of the observed structures. For $\operatorname{Re}=1$, redistribution of particles at the end of each mixing element display the same flow pattern that is shown in Fig. 2.1. Figure 2.1 presents one experimental picture on the mixing in a helical mixer. In that visualization experiment, a two-part epoxy (one part black, the other white) is forced through the mixer and the mixer is then cut into sections revealing the flow and mixing pattern at various axial locations along the mixer. The helical static mixer shows the same mixing behavior for creeping flows and also low Reynolds number, e.g., $\mathrm{Re}=1$, laminar flows and a comparison between the numerical results, shown in this chapter, and the existing experimental results, Fig. 2.1, shows excellent agreement for the numerical results were obtained. Also, it is observed that for low Reynolds number flows, each mixing element splits the flow into two parts and the mixer behavior follows the analytical 
prediction, Eq. 2.7. For the case of $\mathrm{Re}=100$, however, the mixing pattern is far from the pattern obtained for creeping and low Reynolds number laminar flows. The large line shape areas that were distinguished in low Reynolds number flows are replaced with curved areas. Although, like low Reynolds number cases, the particles locations at different flow cross-sections are anti-symmetric after the first element. As the flow passes through the mixer, the curvature of particles locations increases. It can be seen that after the fourth mixing element a large portion of flow cross-section is filled with fluid particles. Also, it can be seen that even numbered mixing elements have more influence on mixing. For the sixth element, however, the particles distribution at the last mixing element does not show any improvement compared to the particles distribution at the fifth element. It can be explained by the fact that after the sixth element flow enters a pipe with no mixer and therefore, empty pipe boundary conditions are applied to the flow. Hence, the last mixing element is not as effective as mixing elements located in the middle of helical static mixer.

For Reynolds numbers of $0.01,0.1,10$, and 1000, the plots of the particle positions at the end of the second, the fourth, and the sixth elements, respectively from top to bottom, are shown in Figs. 5.15-5.18. By observing the mixing patterns at the end of even numbered mixing element for all different Reynolds numbers, it is found that separated islands are distinguishable after the flow passes the second mixing element. Increasing the Reynolds number breaks these few large islands into several smaller islands.

The islands of flow, observed after the second elements in low Reynolds number flows, become divided into narrow line shape regions after the flow passes the fourth element, and can be still recognized after the sixth element at the lower Reynolds 
number. Increasing the Reynolds number breaks the separated areas and therefore increases the mixing of fluid particles in the same cross-section.

\subsubsection{G-value}

For a helical static mixer, the G-value can be calculated from the following:

$$
\mathrm{G} \text { - value }=\frac{1}{2}\left(\frac{\pi D}{V_{m}}\right)^{1 / 2}\left(\frac{\operatorname{Re} \Delta p}{\rho}\right)^{1 / 2}
$$

Although it has been demonstrated that the original derivation of the G-value was flawed for three-dimensional flows $[123,134]$ and cannot be universally applied to different types of mixers or different size mixers, the G-value remains entrenched in the engineering literature and continues to be used [63].

Figure 5.19 shows the calculated G values for different flow conditions for water $\left(\rho=998.2 \mathrm{~kg} / \mathrm{m}^{3}, \mu=0.001003 \mathrm{~kg} / \mathrm{m} . \mathrm{s}\right)$. As can be seen, the G-value increases as the Reynolds number raises. Also the rate of the increasing of the G-value is enhanced when the Reynolds number increases, suggesting that the helical static mixers are not very energy efficient in high Reynolds number flows.

As can be seen from Eq. (5.2), for a given geometry and a specific Reynolds number, the Gvalue is a function of material density and the pressure drop of the flow in the mixer. For a material which its density is $103 \mathrm{~kg} / \mathrm{m}^{3}$, for the case of $\mathrm{Re}=1,000$, the G- 
value is $29,279,317.3570 \mathrm{1} / \mathrm{s}$; however for a material witch its density is $104 \mathrm{~kg} / \mathrm{m}^{3}$ (less than one percent denser material), the G-value is $292.78561 / s$ (for the same Reynolds number), which is $10^{-5}$ times smaller than the first value.

\subsubsection{Residence Time Distribution}

Figures 5.20-5.23 show the distribution function for water, calculated for $\operatorname{Re}=1,10$, 100, and 1000. For all cases, $d t^{*}=0.01$. The distribution functions are similar for $\operatorname{Re}=$ 10 , and $\operatorname{Re}=100$; for $\operatorname{Re}=1$, a similar pattern is still observed. However, for $\operatorname{Re}=1000$, the distribution function is quite different. For all cases the function starts at $t^{*} \approx 0.6$, which means that fluid elements passes the mixing element at a minimum nondimensional time of $t^{*}=0.6$. The maximum non-dimensional residence time is more than 3 for all cases but $\operatorname{Re}=1000$, which is about 1.8 . For $\operatorname{Re}=1$, the maximum non-

dimensional residence time is about 3. Only one mode of distribution function is observed for this case.

By increasing the Reynolds number to 10 and then to 100 , the residence time increases and also a second mode of distribution function is created. For $\operatorname{Re}=10$, the first (second) mode ends (starts) at $t^{*} \approx 2.8$; and for $\operatorname{Re}=100$, the first (second) mode ends (starts) at $t^{*} \approx 2.2$. However, for both cases the second mode is substantially weaker than the first mode. The first mode is associated with streamlines that occupy a large portion of the flow. 
For $\operatorname{Re}=1000$, the majority of fluid particles belong to the first mode, which is associated with a residence time of $0.6 \leq t^{*} \leq 1.2$. The second mode is significantly weaker. This suggests that fluid elements experience the same history in the flow.

\subsubsection{Structure Radius}

Figure 5.24 shows structure radii that were obtained at the end of each mixing element for $\operatorname{Re}=1$. As the flow passes through the mixer, the structure radius decreases. At flow inlet $r_{s}=0.5$; at the end of the first mixing element $r_{s} \approx 0.2$; and at the end of the second element $r_{s} \approx 0.1$. The rate of decrease of the structure radius is greater over the upstream mixing elements; as the number of elements increases their effect on the structure radius decreases.

Structure radii at the end of the last mixing element for different flow Reynolds numbers are shown in Fig. 5.25. It might be expected that by increasing the Reynolds number, the structure radius should decrease. However, although this is true for most cases, it is not correct in general. For $\mathrm{Re}=10$, the radius structure at the last mixing element is larger than the radius structure at the same flow cross-section, when $\operatorname{Re}=1$ or less. This suggests that working fluids are less mixed at this Reynolds number, compared to the case of $\operatorname{Re}=1$. These effects are also recognizable by observing Figs. 5.13, 5.15- 


\subsubsection{Particles Distribution Uniformity}

The perfect mixing of two fluids can be defined as a uniform distribution of all fluid particles in the flowfield cross-section. The value of the PDU function can be used to determine the how close a particular flow comes to this idealization in a given flow crosssection. This function varies between zero (the poorest distribution of mixed fluid particles) and one (a totally uniform distribution of mixed fluid particles). The calculated PDU values for different flow conditions and at the different flowfield cross-sections are shown in Figs. 5.26 and 5.27 for $\operatorname{Re}=1$ and Re 100, respectively, for $N_{p}=501,740$. At flow inlet the PDU value is 0.25 corresponding to the particles which are located uniformly in half of the flow cross-section. As the flow goes through the first mixing element, the PDU value increases to above 0.4 (more than a $60 \%$ increase). PDU value also increases significantly from the first to the second mixing element; for the case of $\mathrm{Re}$ $=1$, PDU increase from 0.4174 to 0.6965 (a $66.86 \%$ increase) and for $\operatorname{Re}=100$, it increases from 0.4335 to 0.6654 (a $53.48 \%$ increase). After the second mixing element PDU values increase slightly as the flow passes through the mixer.

PDU values at the end of the $2^{\text {nd }}$, the $4^{\text {th }}$, and the $6^{\text {th }}$ mixing elements, obtained for different flow Reynolds numbers, are compared together in Fig. 5.28. The PDU values obtained for $\operatorname{Re}=0.01$ are very close to these values for $\operatorname{Re}=0.1$ (not shown in the figure) at each flow cross-section. For the case of $\mathrm{Re}=10$, PDU value decreases at the end of the last mixing element.

For $\mathrm{Re}=0.01$, the PDU is slightly less than 0.7 at the end of the second mixing element. By increasing the Reynolds number from 0.01 to 1 , and from 1 to 10 , and also 
from 10 to 100, the PDU value at the end of the second element is found to decrease. As the flow passes through the mixer, the PDU value slightly decreases at the fourth mixing element for creeping flows and it increases again at the end of the last mixing element. For laminar flows, PDU values increase by increasing the number of the mixing elements. It is recognized that by changing the flow pattern from creeping flow to a higher Reynolds number laminar flow, the rate of increasing of the PDU value, is also increased. It can be seen that by increasing the Reynolds number from Re of the order of $10^{1}$ to Re of the order of $10^{2}$, the rate of increase of PDU value s, based on the number of mixing elements, increases. This suggests that the helical static mixer is more efficient for these Reynolds numbers compared to creeping flows, as it was discussed by Byrde and Sawley [60,61]. Helical static mixer behavior is different for $\operatorname{Re}=10$ and $\operatorname{Re}=1000$. Although the PDU increases from the second mixing element to the fourth element, at the end of the last mixing element PDU decreases. For $\mathrm{Re}=10$, the PDU value at the end of the last element is even less than the PDU at the end of the second mixing element, which is in contrast with the mixer behavior for all other flow Reynolds numbers. It suggests that helical static mixers are less effective for very low Reynolds number (laminar) flows.

Figure 5.29 shows the values of PDU at the $4^{\text {th }}$ mixing element for different Reynolds numbers from $\operatorname{Re}=0.01$ to $\operatorname{Re}=1,000$. As can be seen, the values of PDU is almost the same for creeping flows, it increases as the flow pattern changes from creeping flow to laminar. By increasing the Reynolds number in laminar flows, the PDU increases; moreover, the rate of changes of PDU values is higher, when the Reynolds number increases. 
As expected, the PDU value is only a function of the Reynolds number for a specific static mixer. The density of the material used to calculate the PDU value is $10^{3} \mathrm{~kg} / \mathrm{m}^{3}$. For a material with a density of $10^{4} \mathrm{~kg} / \mathrm{m}^{3}$, for $\mathrm{Re}=1,000$, PDU value changes by $0.2 \%$.

Also, the PDU value is dependent on the accuracy of the solver. For example, for the case of $\operatorname{Re}=100$, the calculated PDU value at the $4^{\text {th }}$ mixing element is $28 \%$ larger when the second order flow solver is used compare to the calculated PDU value based on the obtained results of the first order flow solver. Moreover, it may worthwhile noting that the obtained PDU value is not very sensitive to the value of $N_{p}$, when $N_{p}$ is large enough. For example, for $N_{p}=195,784,501,740$ and 784,282 , the obtained values for PDU at the end of the $2^{\text {nd }}$ mixing element for $\operatorname{Re}=1,000$ is $99.7 \%, 99.8 \%$, and $100 \%$, respectively, of the calculated PDU value when $N_{p}=1,225,784$. However, for $N_{p}=$ 31,180, the obtained value for PDU is $97.4 \%$ of the calculated PDU value when $N_{p}=$ $1,225,784$.

\subsubsection{Helical Static Mixer Efficiency}

The efficiency of an industrial helical static mixer can be defined based on the energy required for the flow of materials through the system and on the degree of mixing of those materials. The static mixer efficiency can be determined using the following equation: 


$$
e f f=\frac{P D U}{\Delta \hat{P}}
$$

The PDU value at the last mixing element is used to calculate the mixer efficiency. Table 5.4 shows the static mixer efficiency for different working conditions. 


\begin{tabular}{|c|c|c|c|c|c|c|}
\cline { 2 - 7 } \multicolumn{1}{c|}{} & \multicolumn{3}{c|}{ Averaged axial velocity } & \multicolumn{3}{c|}{ Averaged velocity magnitude } \\
\hline $\operatorname{Re}$ & 2nd & 4th & 6th & 2nd & 4th & 6th \\
\hline 1 & 0.000278 & 0.000278 & 0.000278 & 0.000307 & 0.000307 & 0.000306 \\
\hline 10 & 0.002777 & 0.002779 & 0.002123 & 0.003078 & 0.003077 & 0.002457 \\
\hline 100 & 0.027689 & 0.027698 & 0.021245 & 0.038384 & 0.037648 & 0.029405 \\
\hline 1,000 & 0.275793 & 0.2761991 & 0.2125812 & 0.5210491 & 0.4995831 & 0.4377990 \\
\hline
\end{tabular}

Table 5.1: Mean axial velocities and velocity magnitudes $(\mathrm{m} / \mathrm{s})$ 


\begin{tabular}{|c|c|c|c|c|c|c|}
\cline { 2 - 8 } \multicolumn{1}{c|}{} & \multicolumn{7}{c|}{ Mean vorticity } \\
\hline $\operatorname{Re}$ & 1 1st & 2nd & 3rd & 4th & 5th & 6th \\
\hline 1 & $2.3805 \mathrm{E} 0$ & $2.4168 \mathrm{E} 0$ & $2.3962 \mathrm{E} 0$ & $2.4014 \mathrm{E} 0$ & $2.4102 \mathrm{E} 0$ & $9.3000 \mathrm{E}-1$ \\
\hline 10 & $2.2573 \mathrm{E} 1$ & $2.2922 \mathrm{E} 1$ & $2.2750 \mathrm{E} 1$ & $2.2842 \mathrm{E} 1$ & $2.2866 \mathrm{E} 1$ & $9.0665 \mathrm{E} 0$ \\
\hline 100 & $3.0022 \mathrm{E} 2$ & $3.0912 \mathrm{E} 2$ & $3.0375 \mathrm{E} 2$ & $3.0324 \mathrm{E} 2$ & $3.0681 \mathrm{E} 2$ & $1.1613 \mathrm{E} 2$ \\
\hline 1000 & $5.1011 \mathrm{E} 3$ & $5.1899 \mathrm{E} 3$ & $4.8749 \mathrm{E} 3$ & $4.7803 \mathrm{E} 3$ & $4.8907 \mathrm{E} 3$ & $2.1936 \mathrm{E} 3$ \\
\hline & \multicolumn{7}{c|}{ Maximum vorticity } \\
\hline $\operatorname{Re}$ & 1 1st & 2nd & 3rd & 4th & 5 th & 6th \\
\hline 1 & $1.1626 \mathrm{E} 1$ & $1.1794 \mathrm{E} 1$ & $1.1684 \mathrm{E} 1$ & $1.1803 \mathrm{E} 1$ & $1.1966 \mathrm{E} 1$ & $1.1294 \mathrm{E} 1$ \\
\hline 10 & $1.1621 \mathrm{E} 2$ & $1.1860 \mathrm{E} 2$ & $1.1518 \mathrm{E} 2$ & $1.1808 \mathrm{E} 2$ & $1.1981 \mathrm{E} 2$ & $9.0661 \mathrm{E} 1$ \\
\hline 100 & $1.2549 \mathrm{E} 3$ & $1.3304 \mathrm{E} 3$ & $1.3151 \mathrm{E} 3$ & $1.2876 \mathrm{E} 3$ & $1.3112 \mathrm{E} 3$ & $6.9353 \mathrm{E} 2$ \\
\hline 1000 & $2.4660 \mathrm{E} 4$ & $2.2002 \mathrm{E} 4$ & $2.3740 \mathrm{E} 4$ & $2.0511 \mathrm{E} 4$ & $2.3757 \mathrm{E} 4$ & $1.1642 \mathrm{E} 4$ \\
\hline
\end{tabular}

Table 5.2: Vorticity magnitude at each mixing element 


\begin{tabular}{|c|c|}
\hline $\begin{array}{c}\text { Reynolds } \\
\text { number }\end{array}$ & $\begin{array}{c}\text { Pressure drop } \\
\text { ratio }\left(\Delta P^{*}\right)\end{array}$ \\
\hline 1 & 15.2061 \\
\hline 10 & 15.4197 \\
\hline 100 & 29.4601 \\
\hline 1000 & 87.3638 \\
\hline
\end{tabular}

Table 5.3: Pressure drop ratio for a six-element helical static mixer 


\begin{tabular}{|c|c|}
\hline $\begin{array}{c}\text { Reynolds } \\
\text { number }\end{array}$ & Efficiency \\
\hline 0.1 & 0.0501 \\
\hline 1 & 0.0471 \\
\hline 10 & 0.0432 \\
\hline 100 & 0.0250 \\
\hline 1000 & 0.0092 \\
\hline
\end{tabular}

Table 5.4: Helical Static Mixer Efficiency 


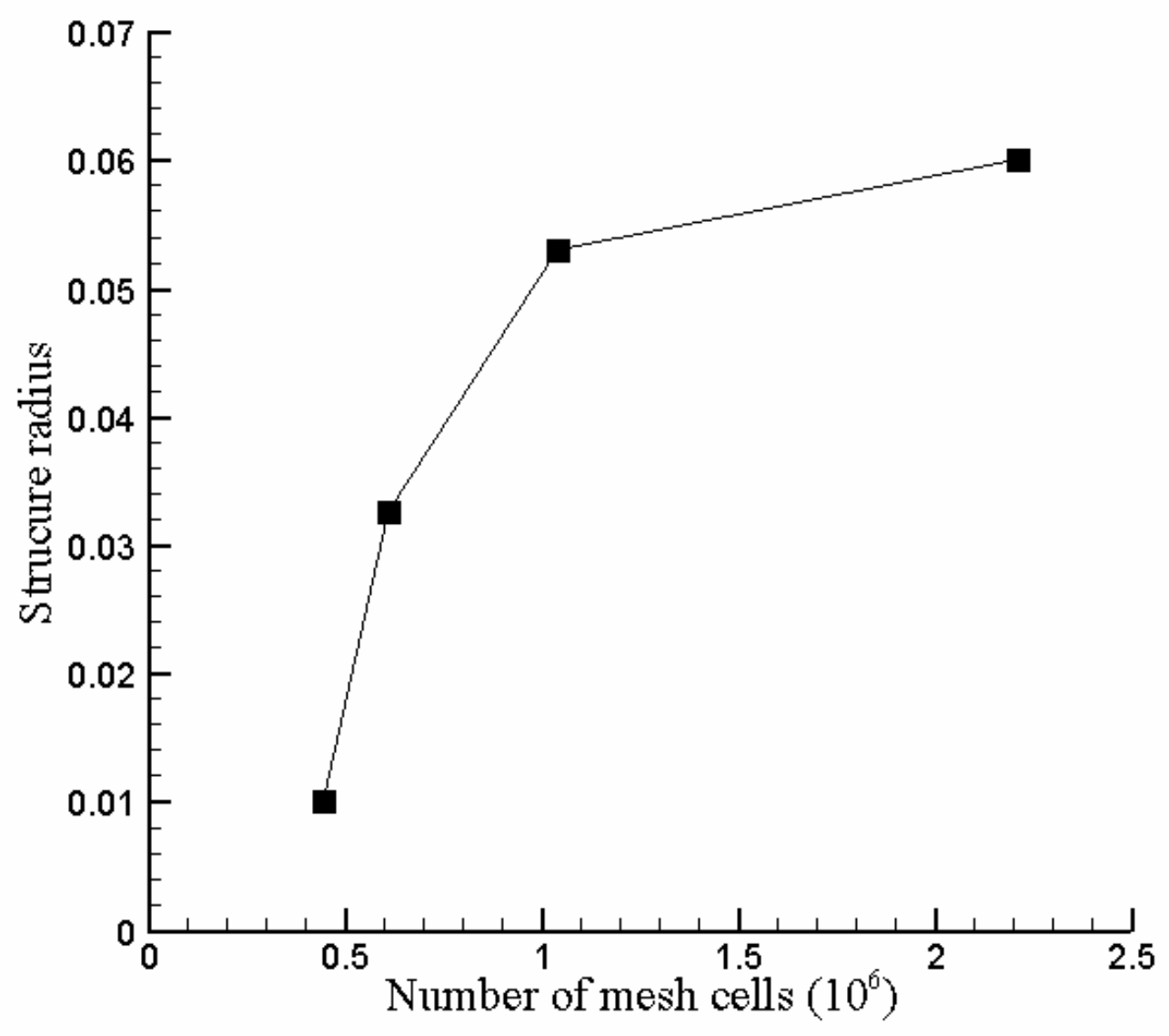

Figure 5.1a: The obtained structure radius for $\mathrm{Re}=10$ 


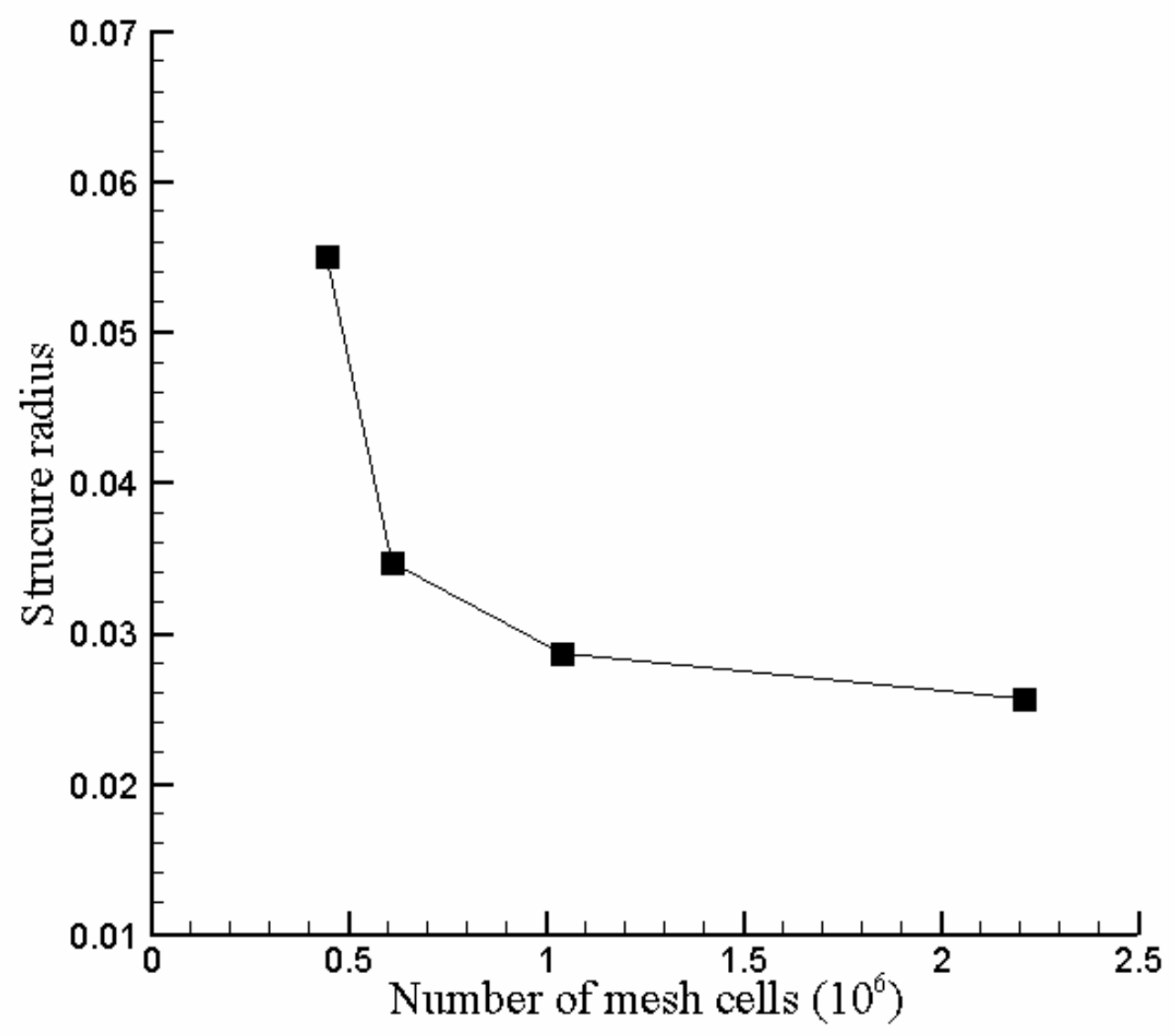

Figure 5.1b: The obtained structure radius for $\mathrm{Re}=100$ 


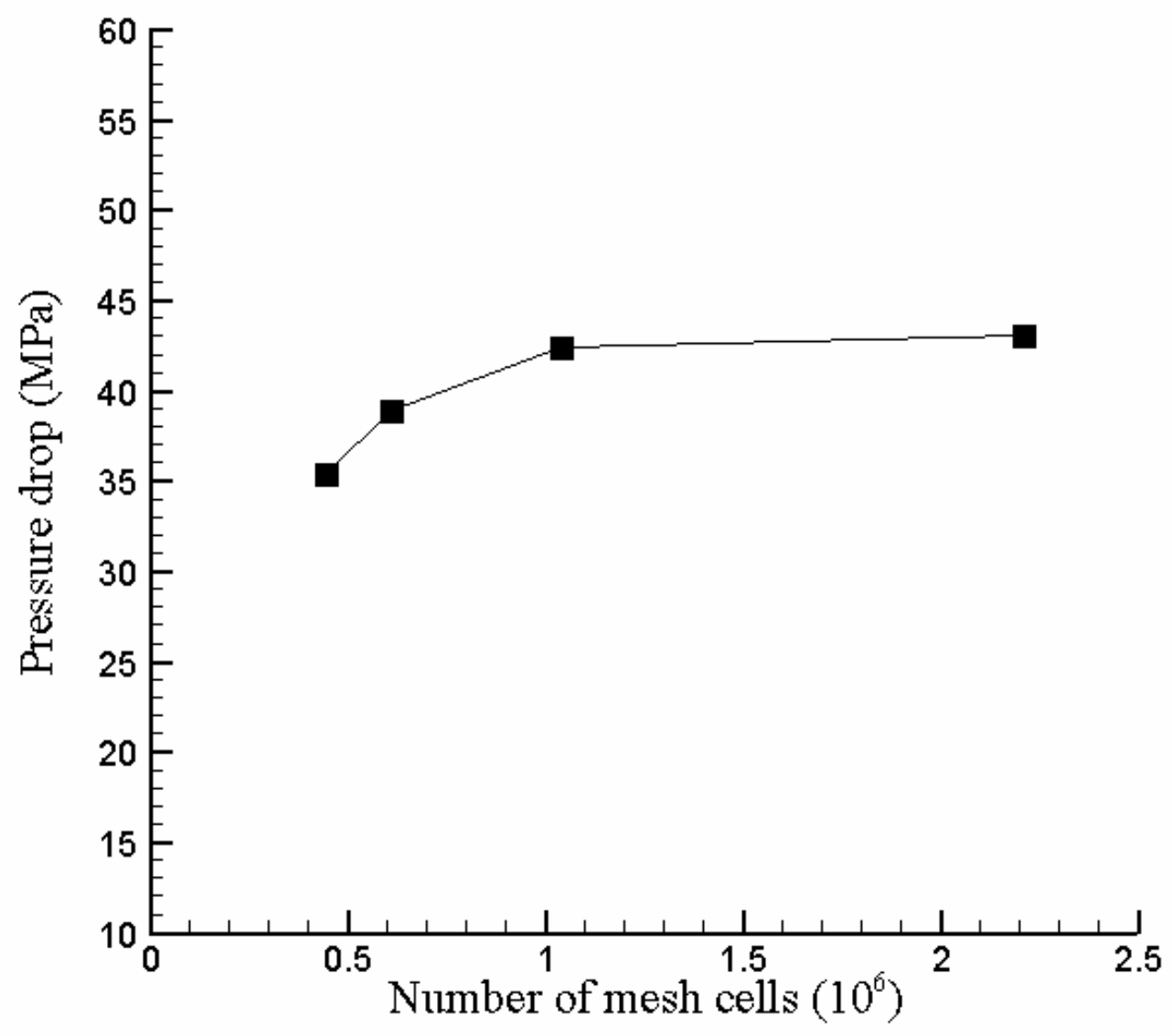

Figure 5.2a: The computed pressure drop for $\operatorname{Re}=10$ 


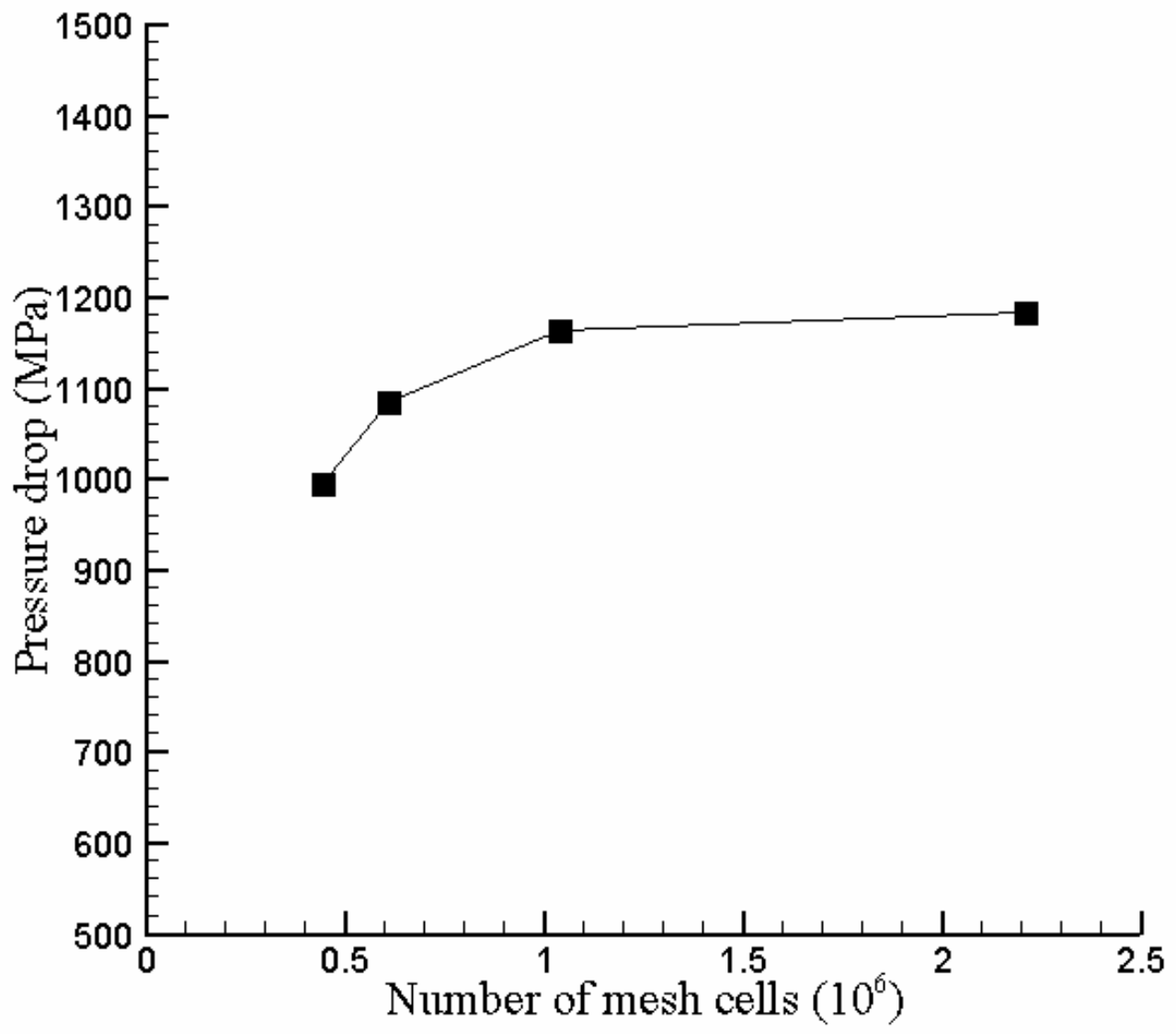

Figure 5.2b: The computed pressure drop for $\operatorname{Re}=100$ 

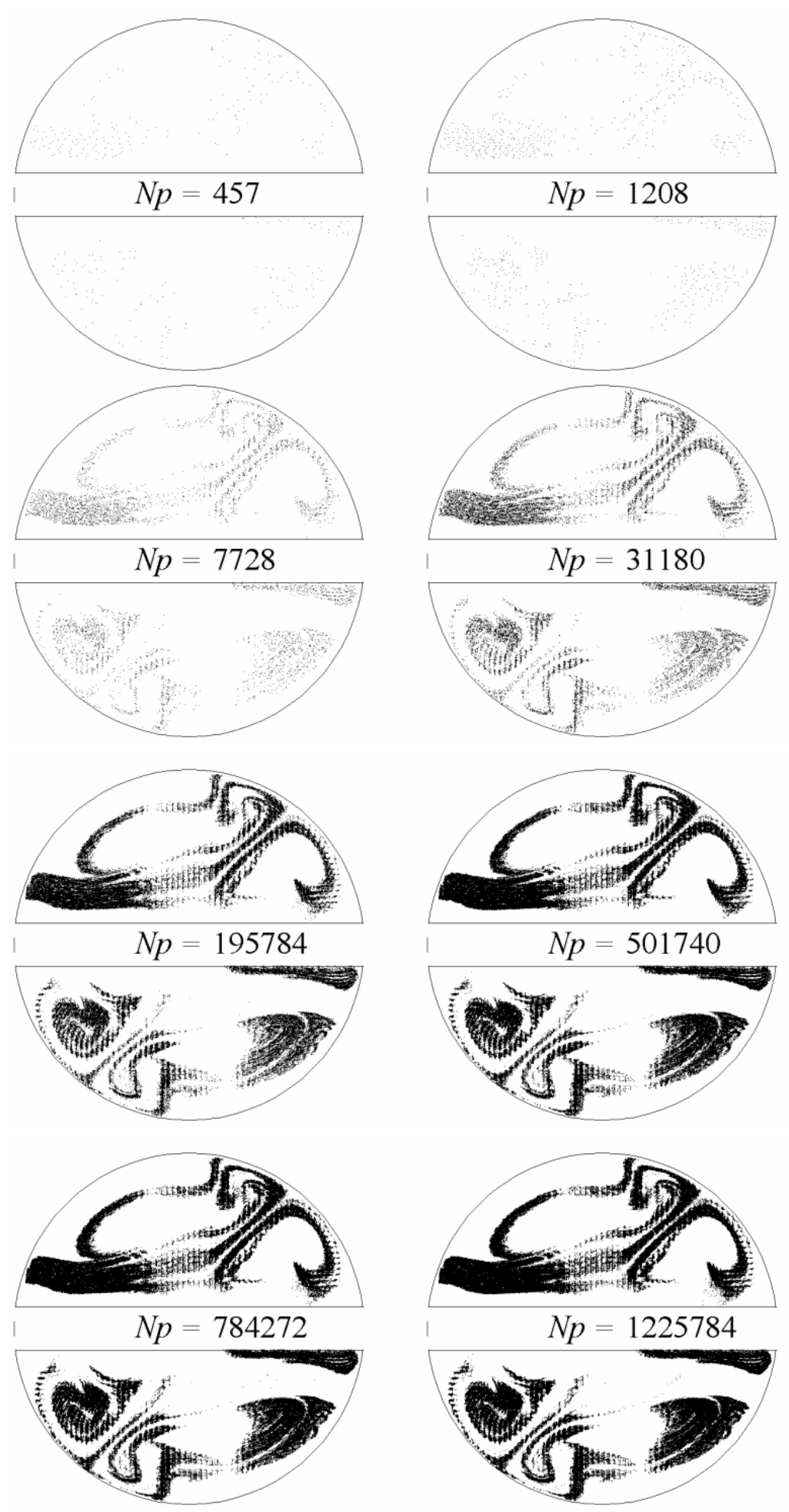

Figure 5.3: Images of particle distribution at $2^{\text {nd }}$ element for $\operatorname{Re}=1000$ 

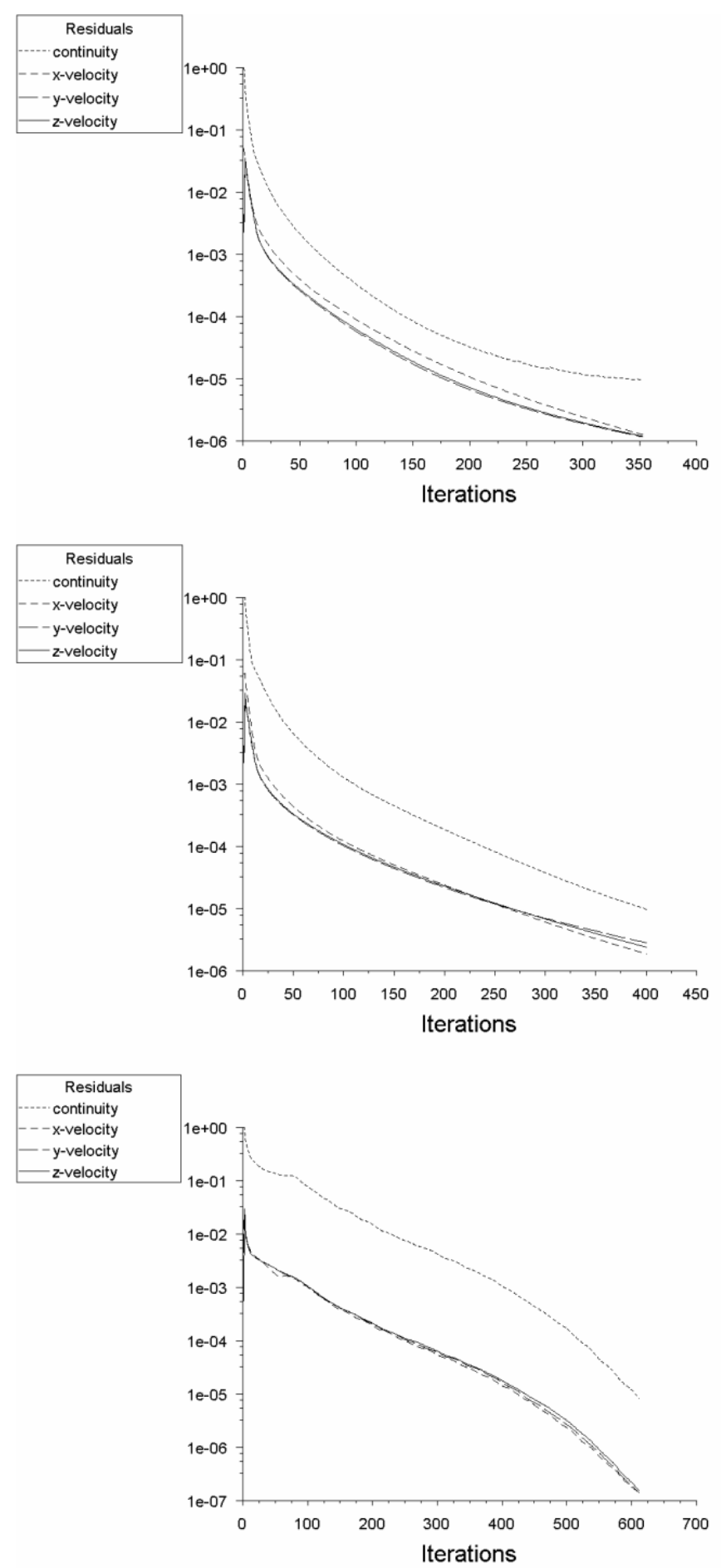

Figure 5.4: Residual curves, from top to bottom: $\operatorname{Re}=0.01,10$, and 1000 

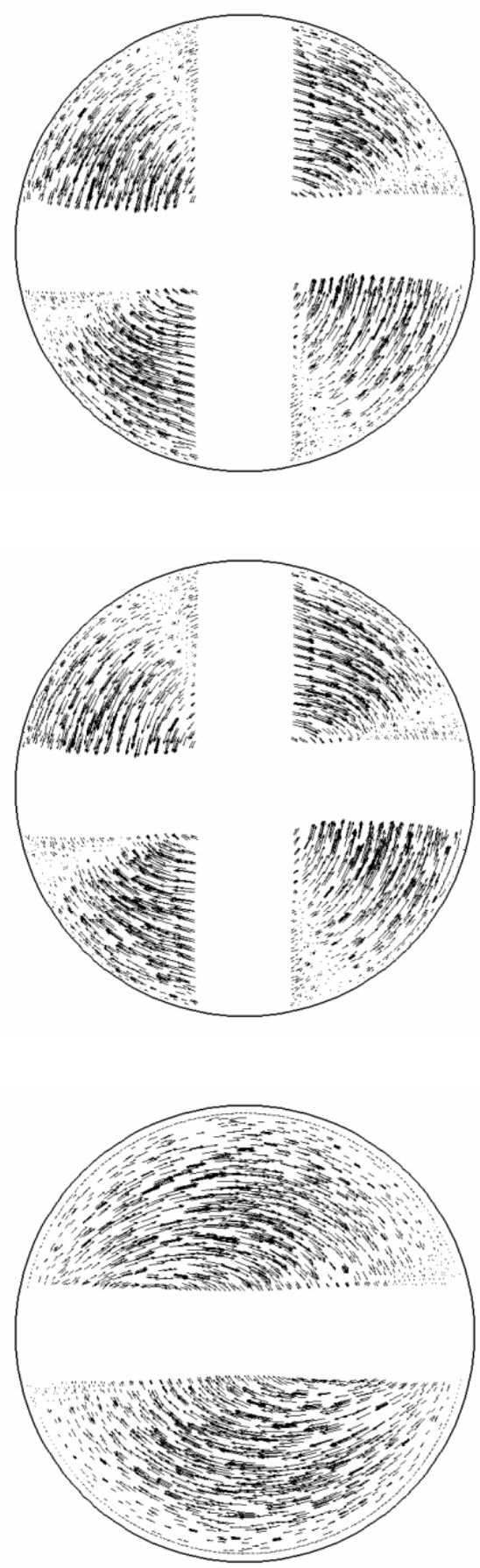

Figure 5.5: Velocity field, from top to bottom: at 2nd, 4th, and 6th mixing elements $(\operatorname{Re}=1)$ 

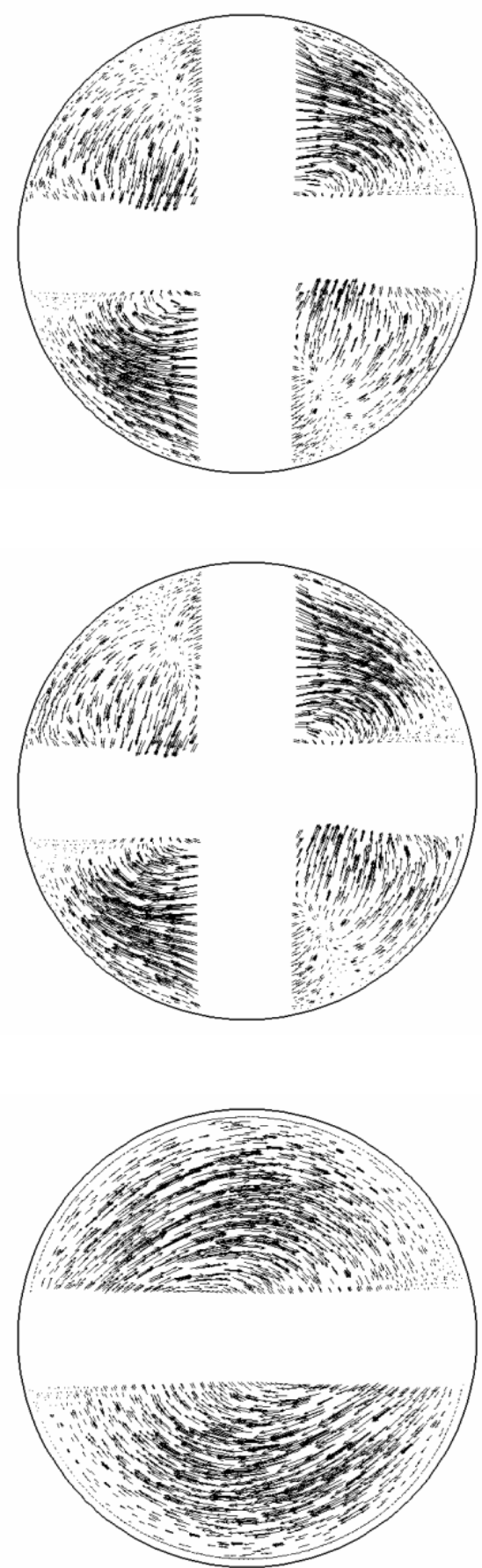

Figure 5.6: Velocity field, from top to bottom: 2 nd, 4 th, and 6th mixing elements $(\operatorname{Re}=10)$ 

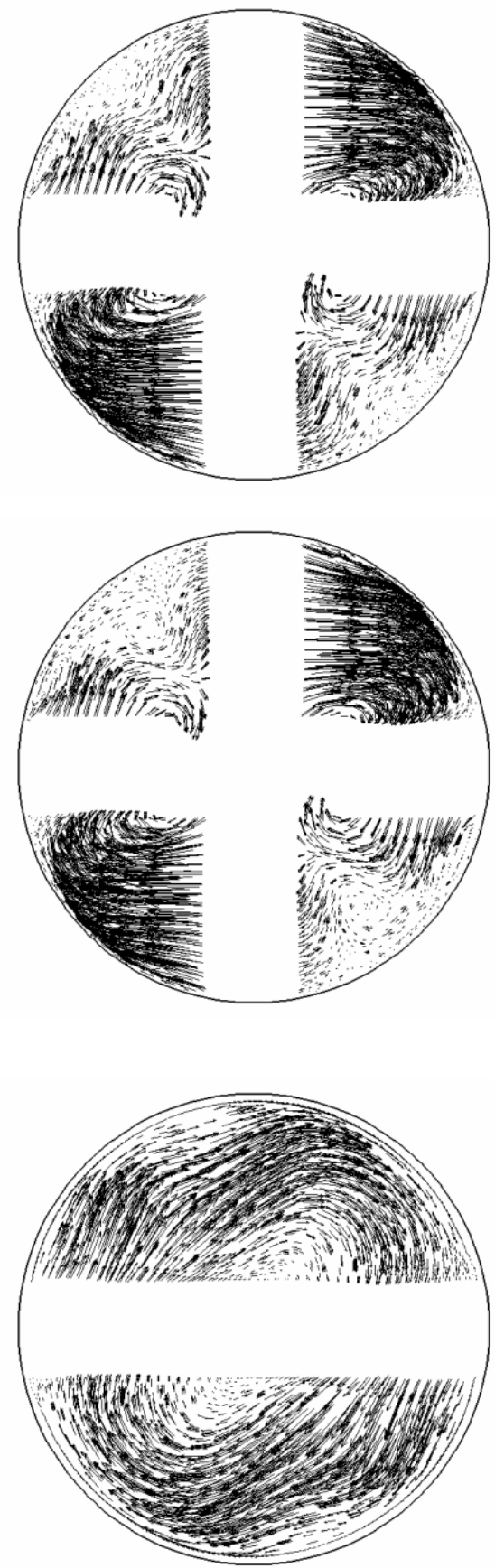

Figure 5.7: Velocity field, from top to bottom: at 2nd, 4th, and 6th mixing elements

$$
(\operatorname{Re}=100)
$$



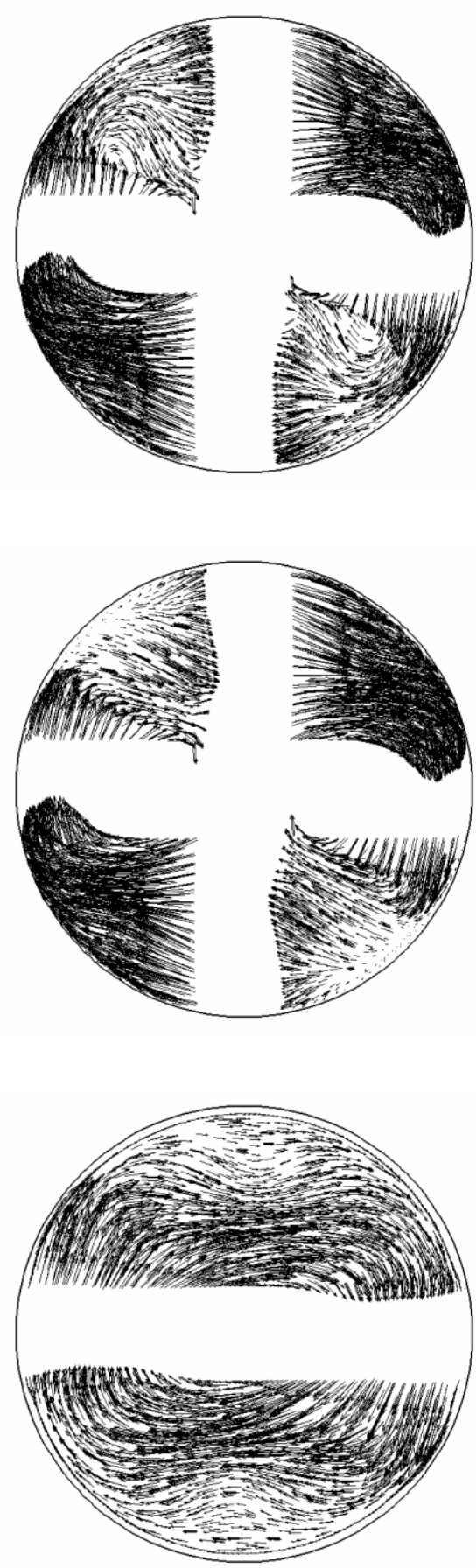

Figure 5.8: Velocity field, from top to bottom: at $2 \mathrm{nd}, 4 \mathrm{th}$, and 6th mixing elements $(\operatorname{Re}=1000)$ 

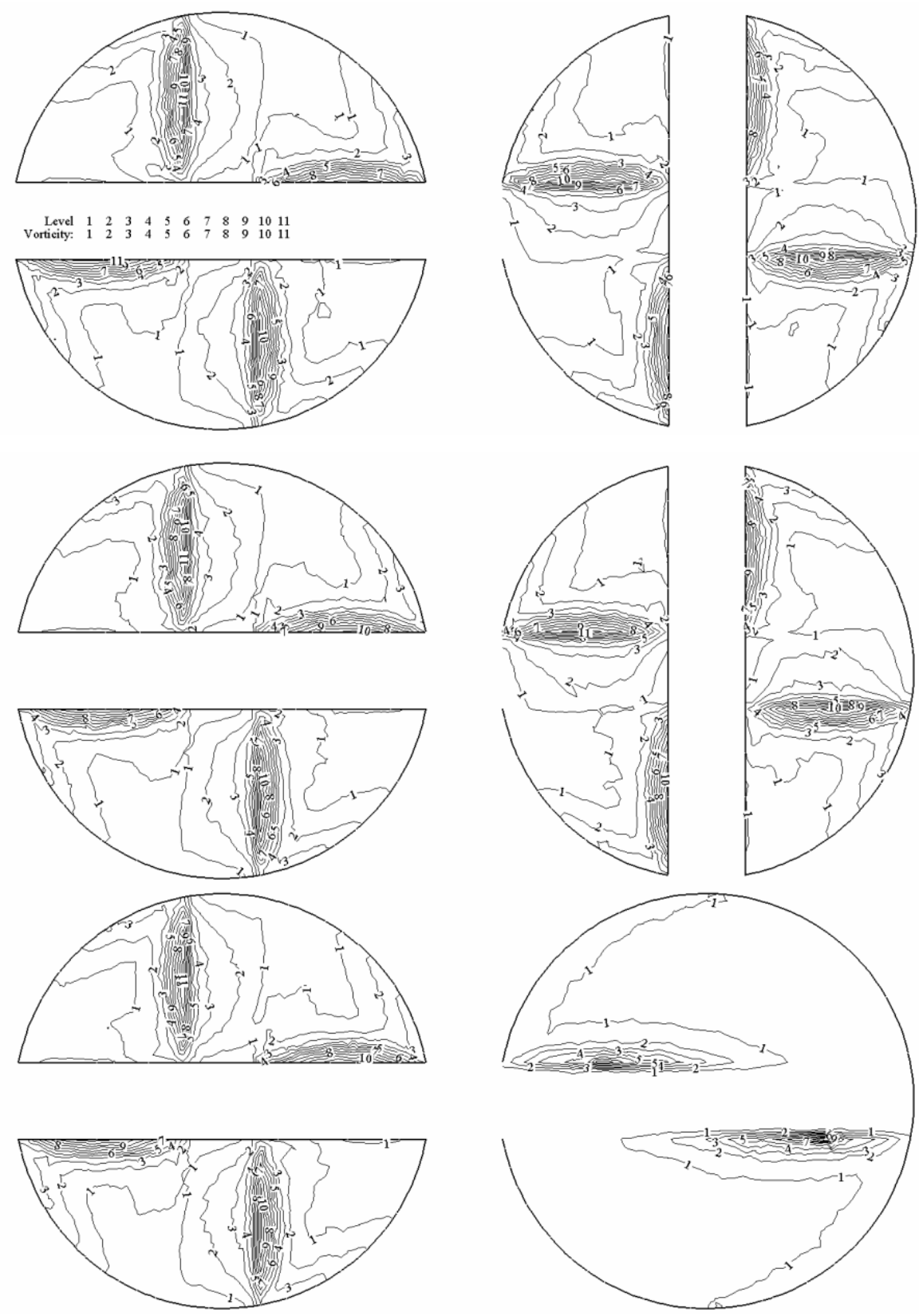

Figure 5.9: Vorticity contours ( $1 / \mathrm{s}$ ), from left to right, top to bottom: at 1st, 2nd,

3rd, 4th, 5th, and 6th mixing elements $(\operatorname{Re}=1)$ 

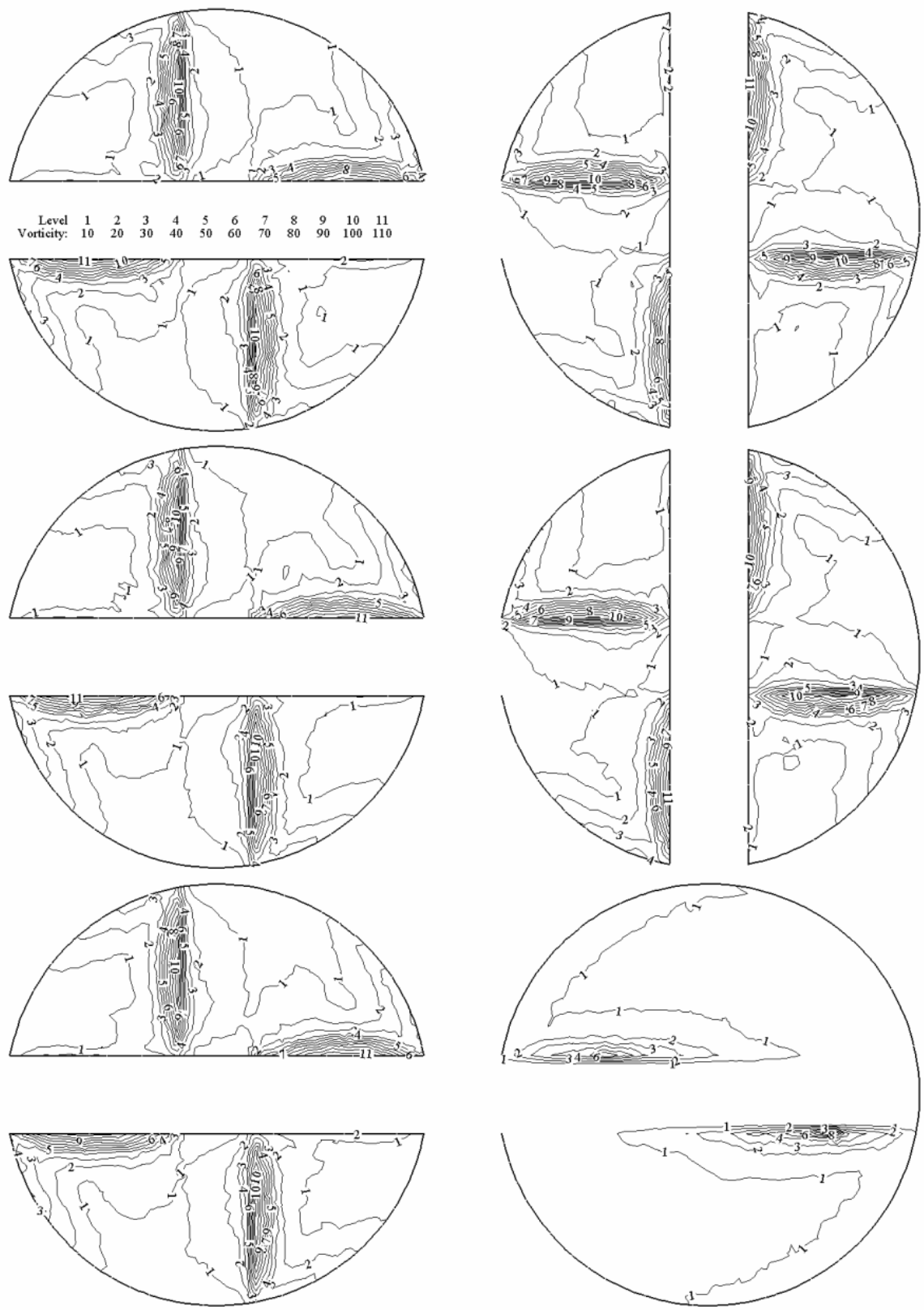

Figure 5.10: Vorticity contours ( $1 / \mathrm{s}$ ), from left to right, top to bottom: at 1st, 2nd, 3rd, 4th, 5th, and 6th mixing elements $(\operatorname{Re}=10)$ 

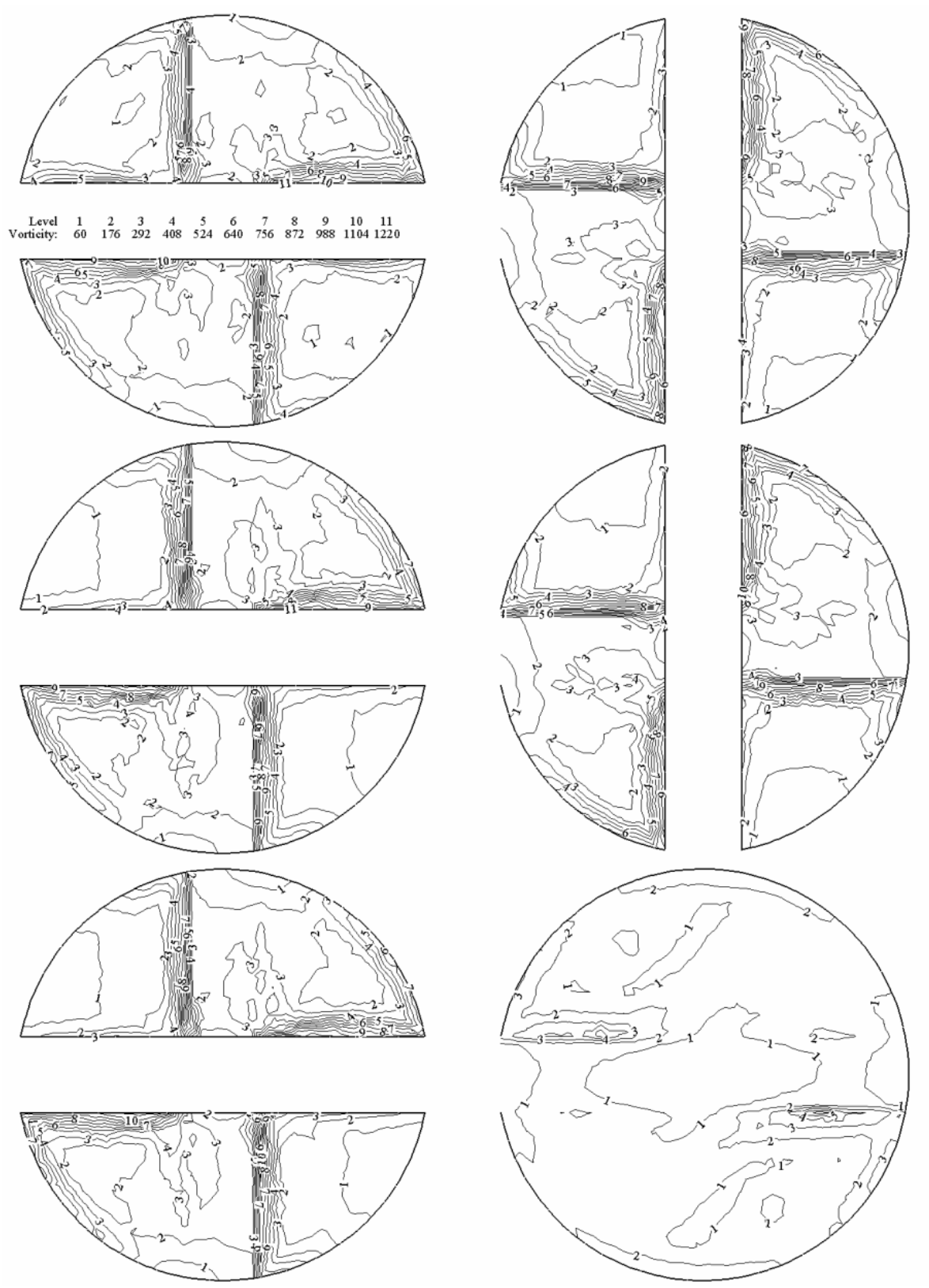

Figure 5.11: Vorticity contours ( $1 / \mathrm{s}$ ), from left to right, top to bottom: at 1st, 2nd, 3rd, 4th, 5th, and 6th mixing elements $(\operatorname{Re}=100)$ 

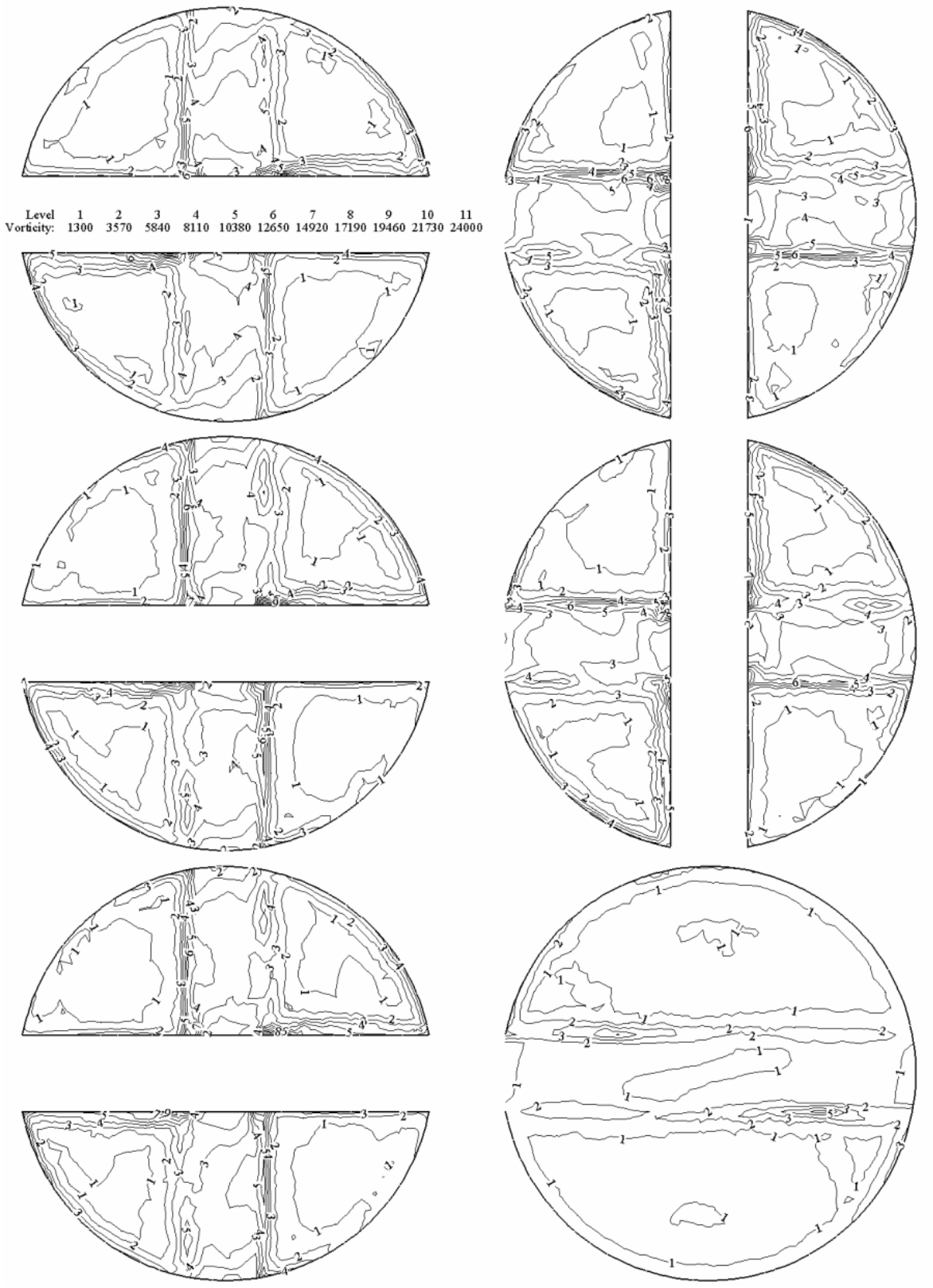

Figure 5.12: Vorticity contours ( $1 / s$ ), from left to right, top to bottom: at 1st, 2nd,

3rd, 4th, 5th, and 6th mixing elements $(\operatorname{Re}=1000)$ 

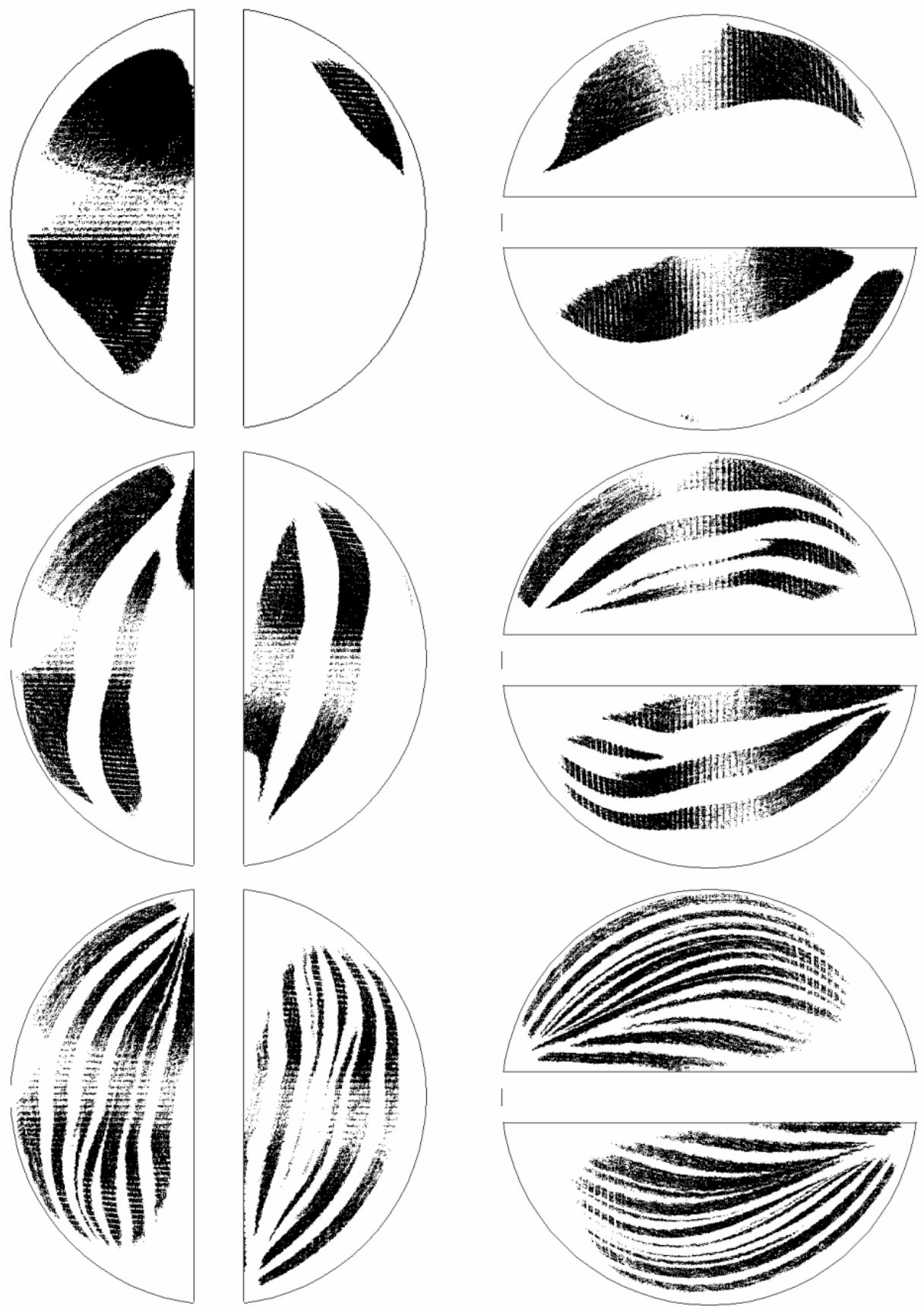

Figure 5.13: Particles locations at each mixing element $(\operatorname{Re}=1)$ 

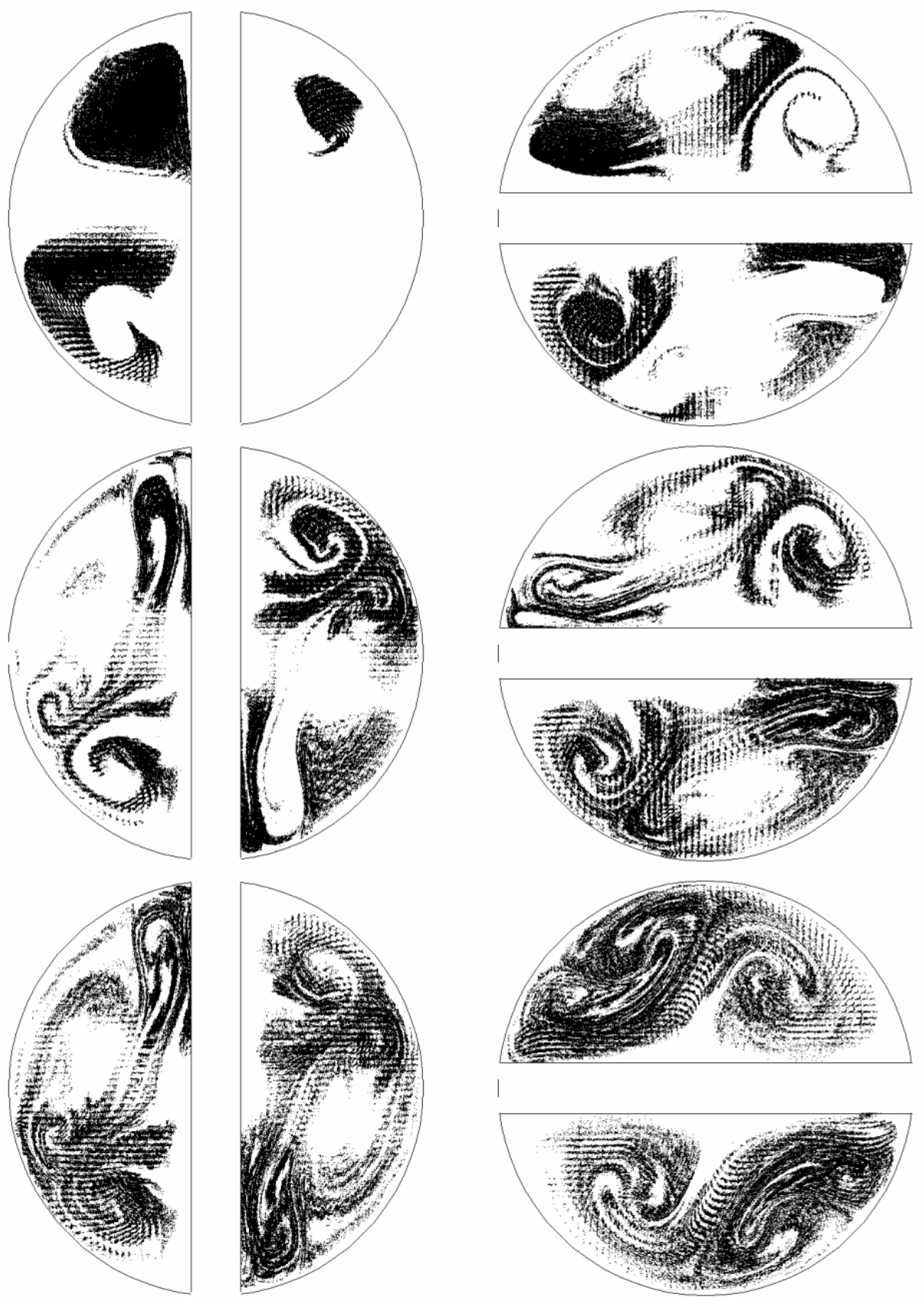

Figure 5.14: Particles locations at each mixing element $(\operatorname{Re}=100)$ 

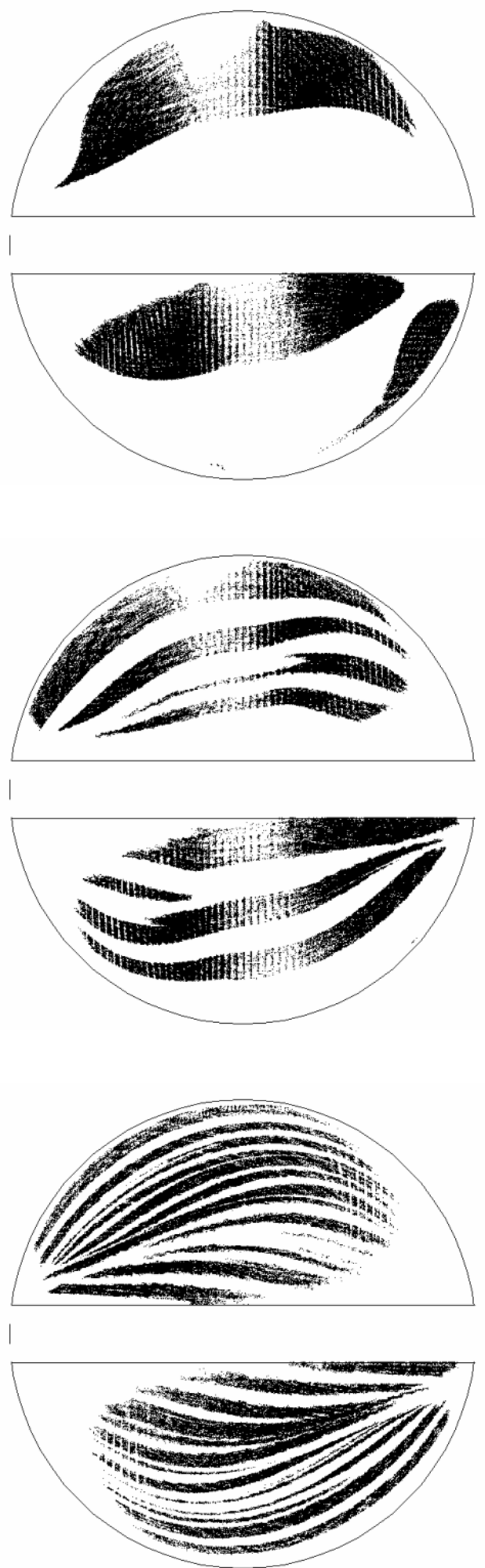

Figure 5.15: Particles locations at 2nd, 4th, and 6th elements $(\operatorname{Re}=0.01)$ 

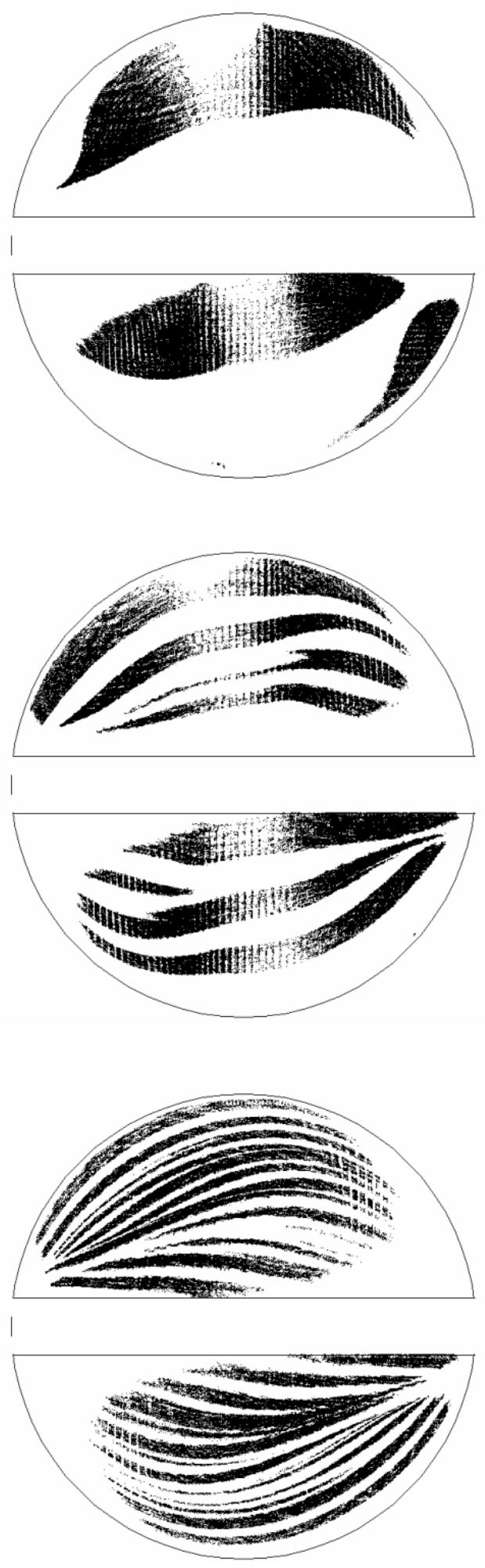

Figure 5.16: Particles locations at 2nd, 4th, and 6th elements $(\operatorname{Re}=0.1)$ 

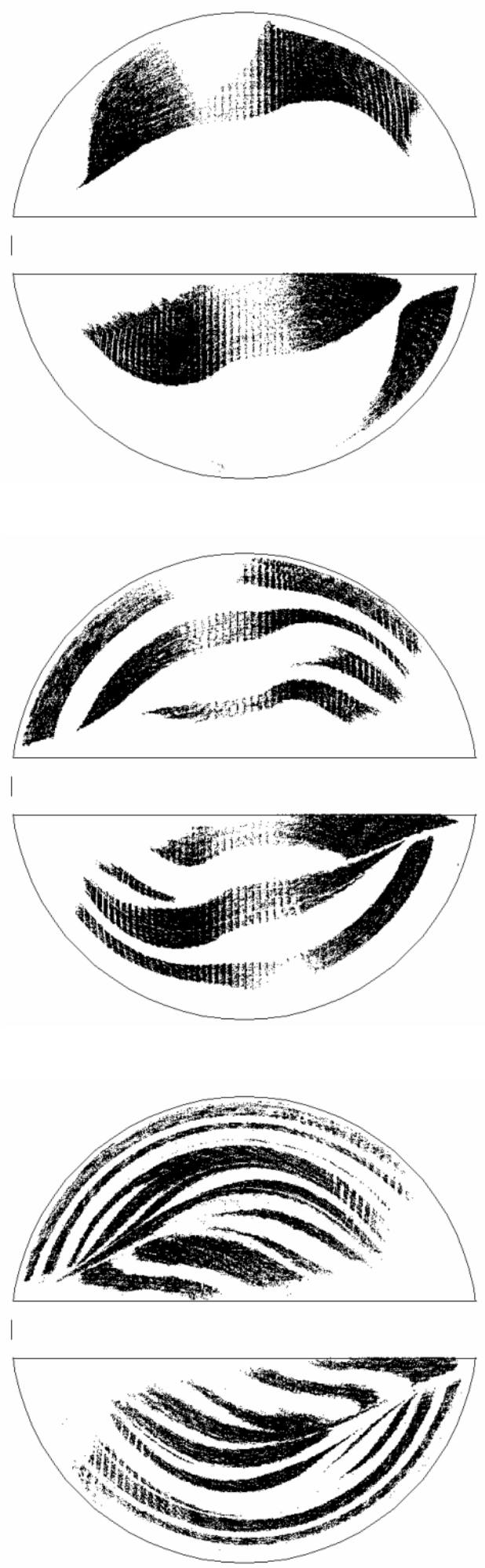

Figure 5.17: Particles locations at 2nd, 4th, and 6th elements $(\operatorname{Re}=10)$ 

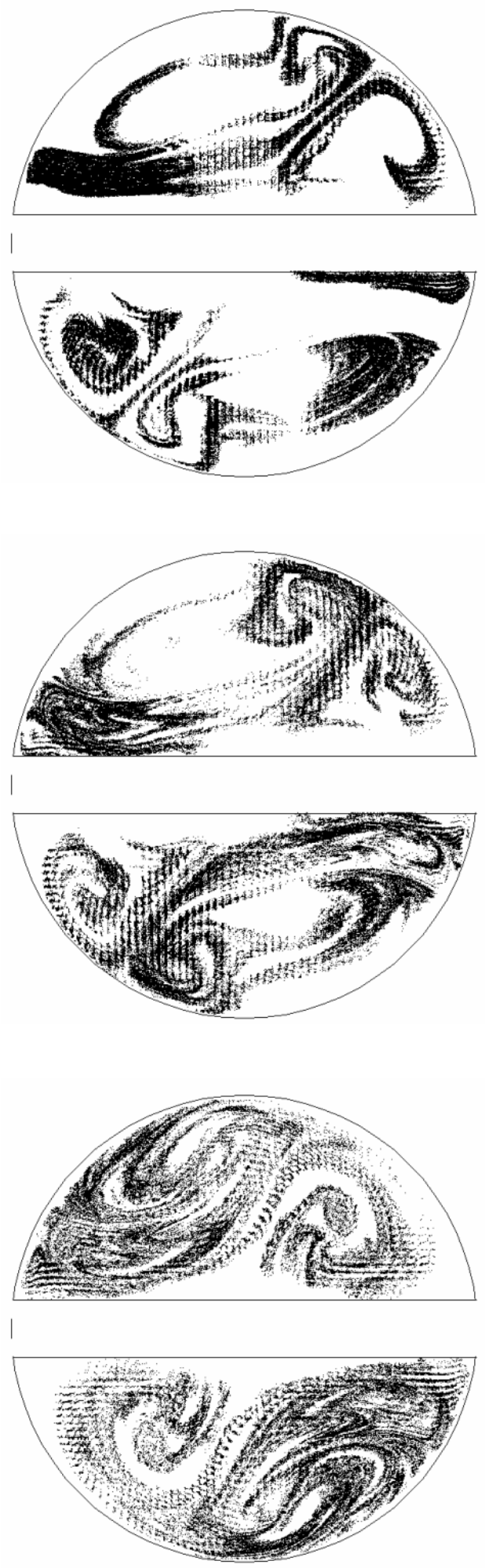

Figure 5.18: Particles locations at 2nd, 4th, and 6th elements $(\operatorname{Re}=1000)$ 


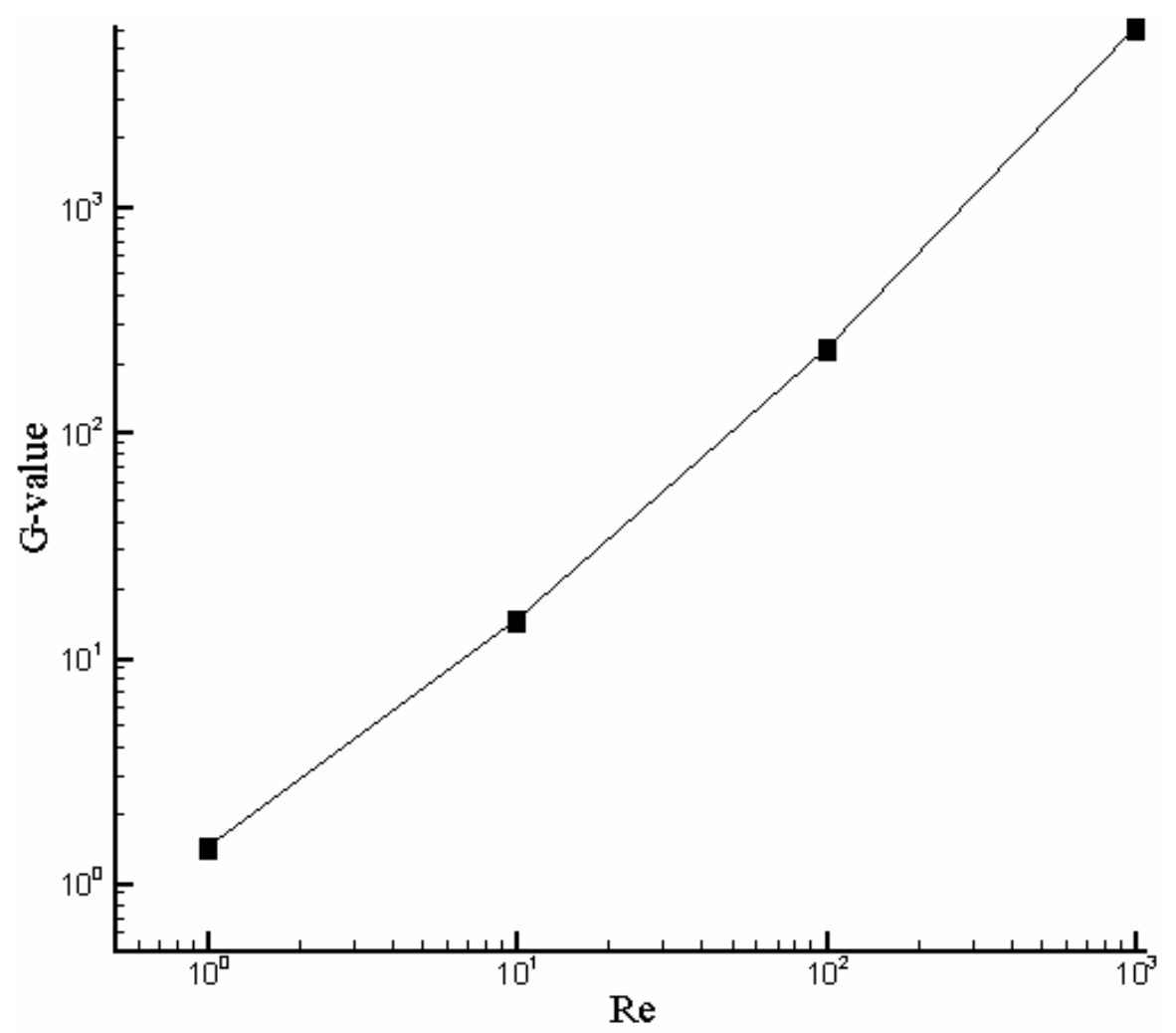

Figure 5.19: G-value ( $1 / s$ ) 


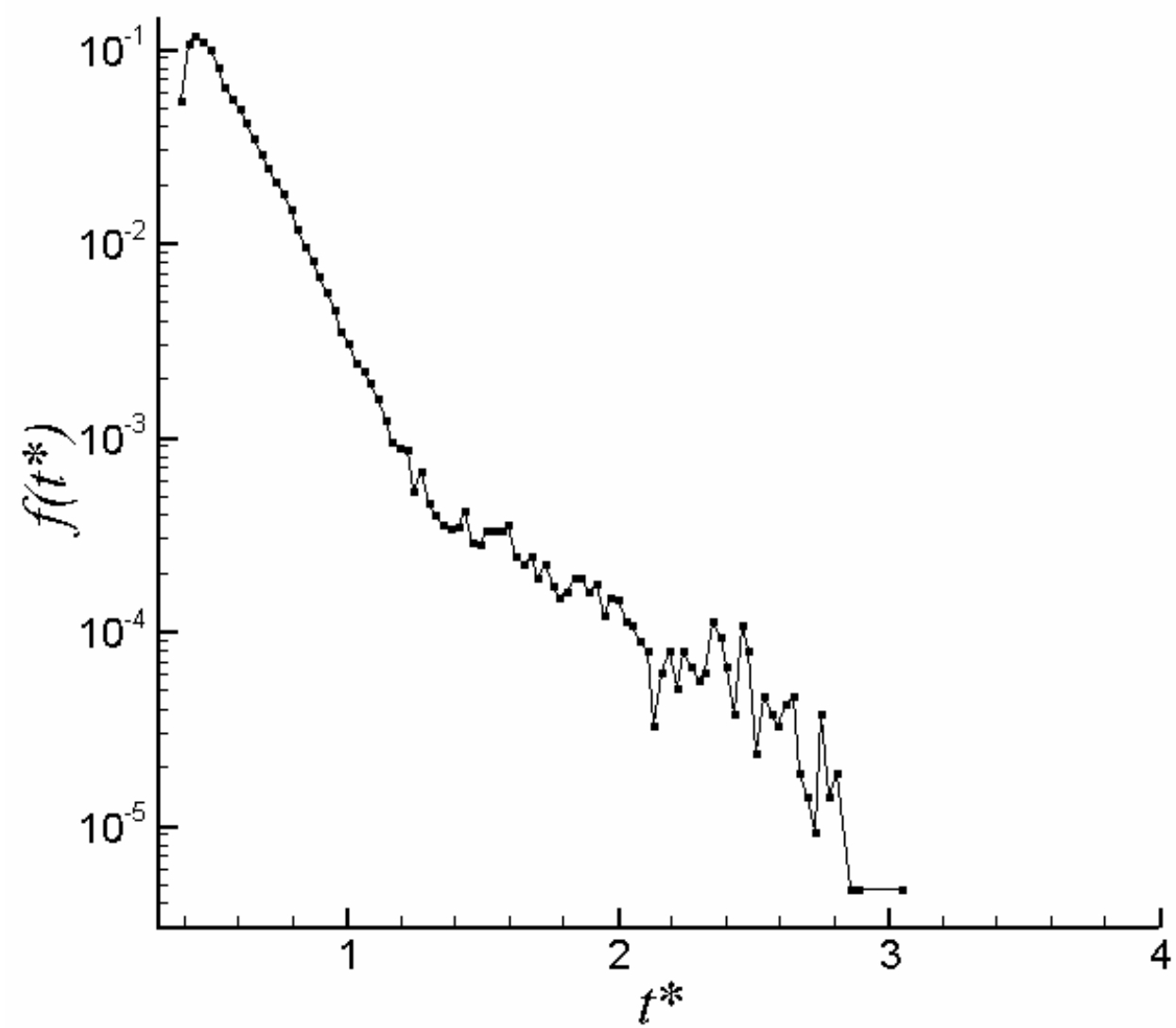

Figure 5.20: Distribution function in a six-element mixer, $d t^{*}=0.01$

$$
(\operatorname{Re}=1)
$$




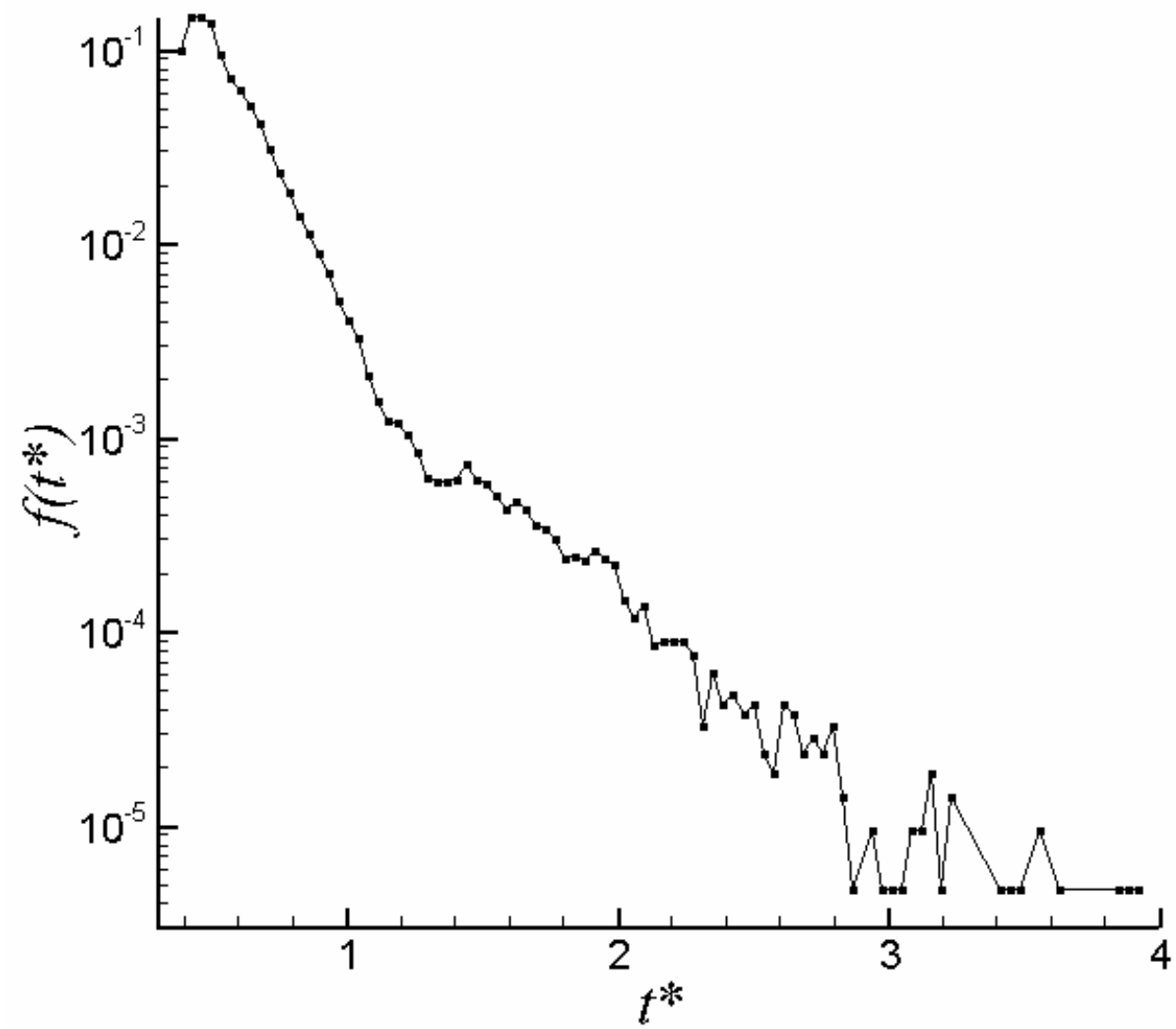

Figure 5.21: Distribution function in a six-element mixer, $d t^{*}=0.01$

$$
(\operatorname{Re}=10)
$$




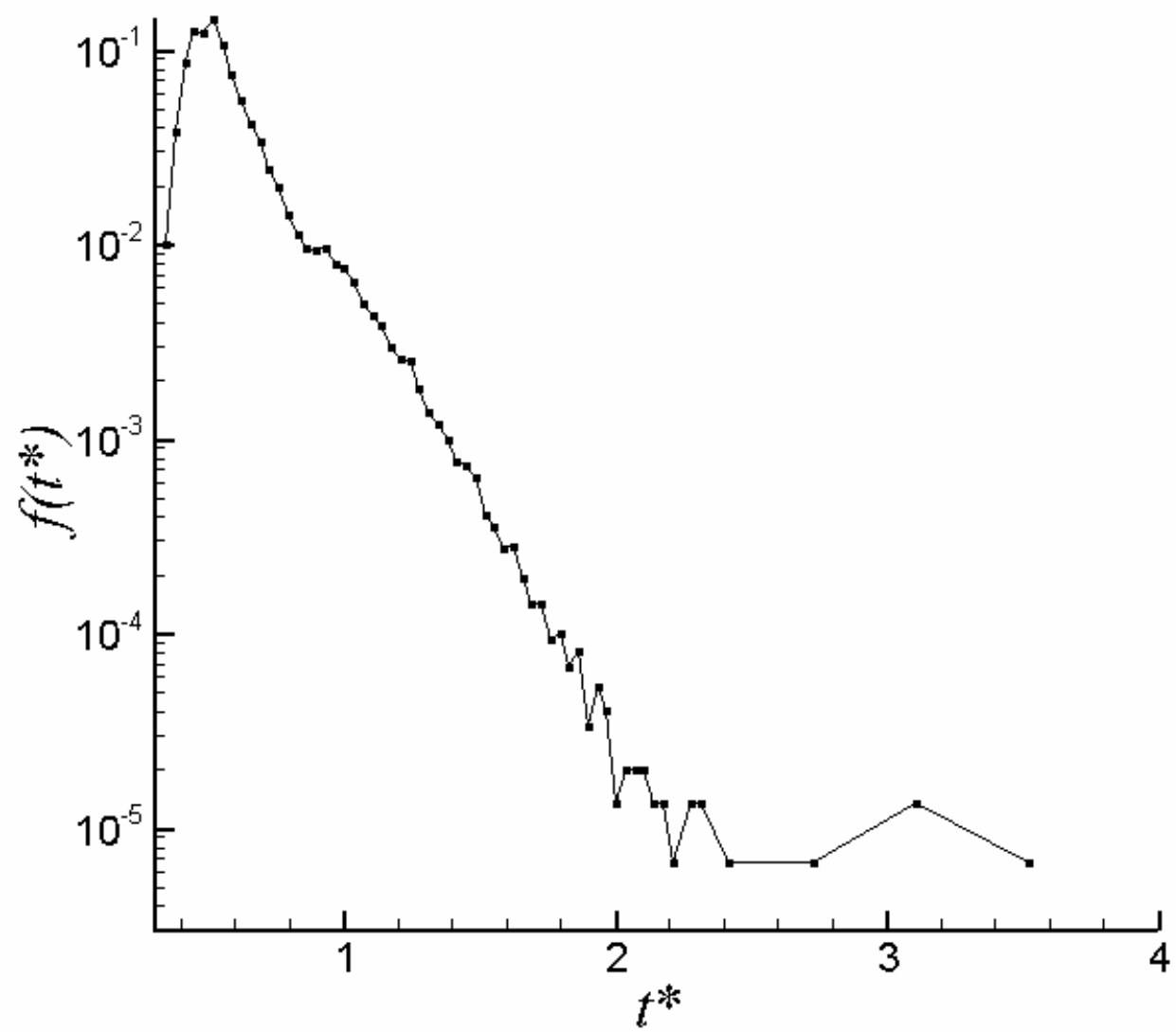

Figure 5.22: Distribution function in a six-element mixer, $d t^{*}=0.01$

$$
(\operatorname{Re}=100)
$$




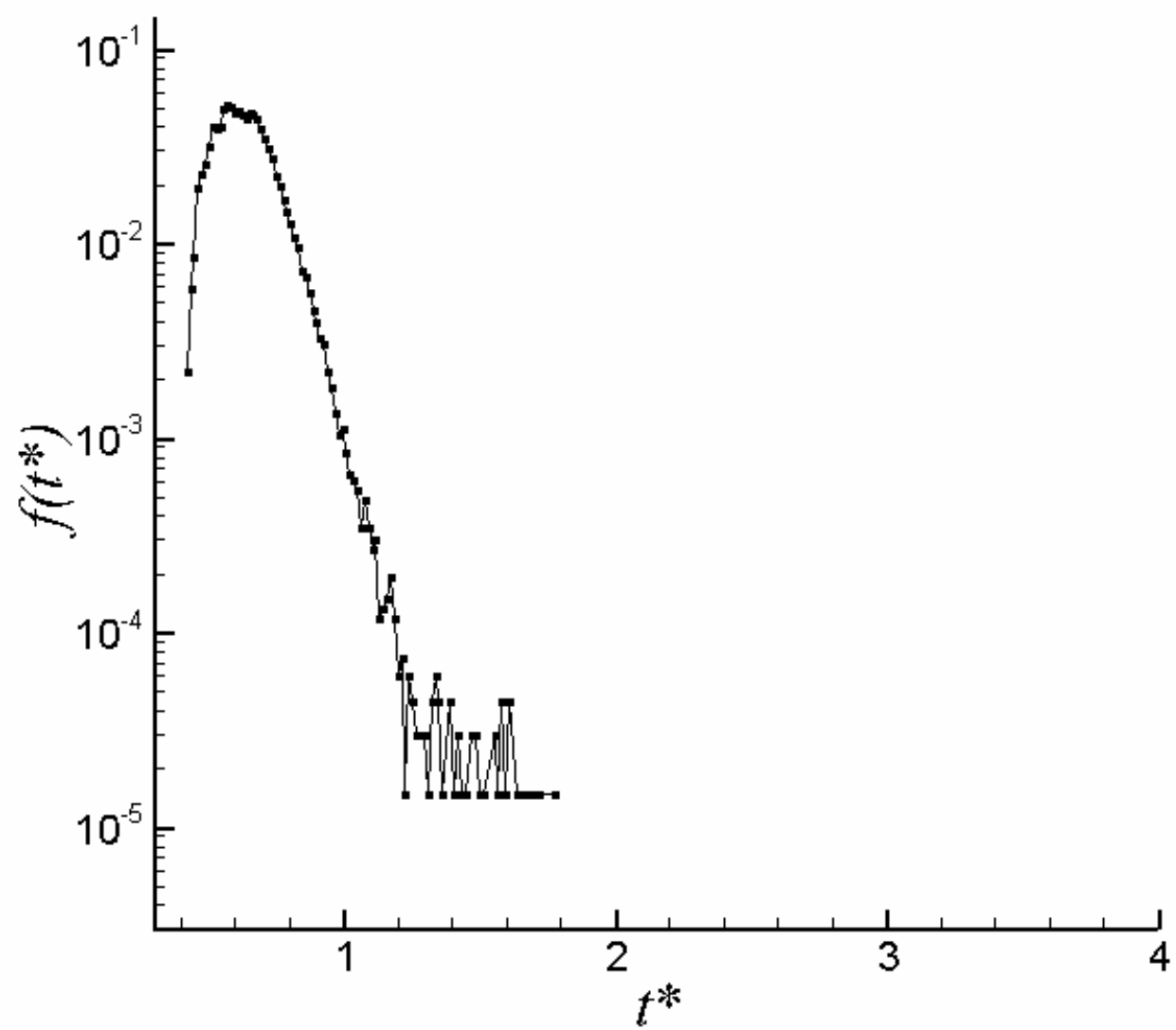

Figure 5.23: Distribution function in a six-element mixer, $d t^{*}=0.01$

$$
(\operatorname{Re}=1000)
$$




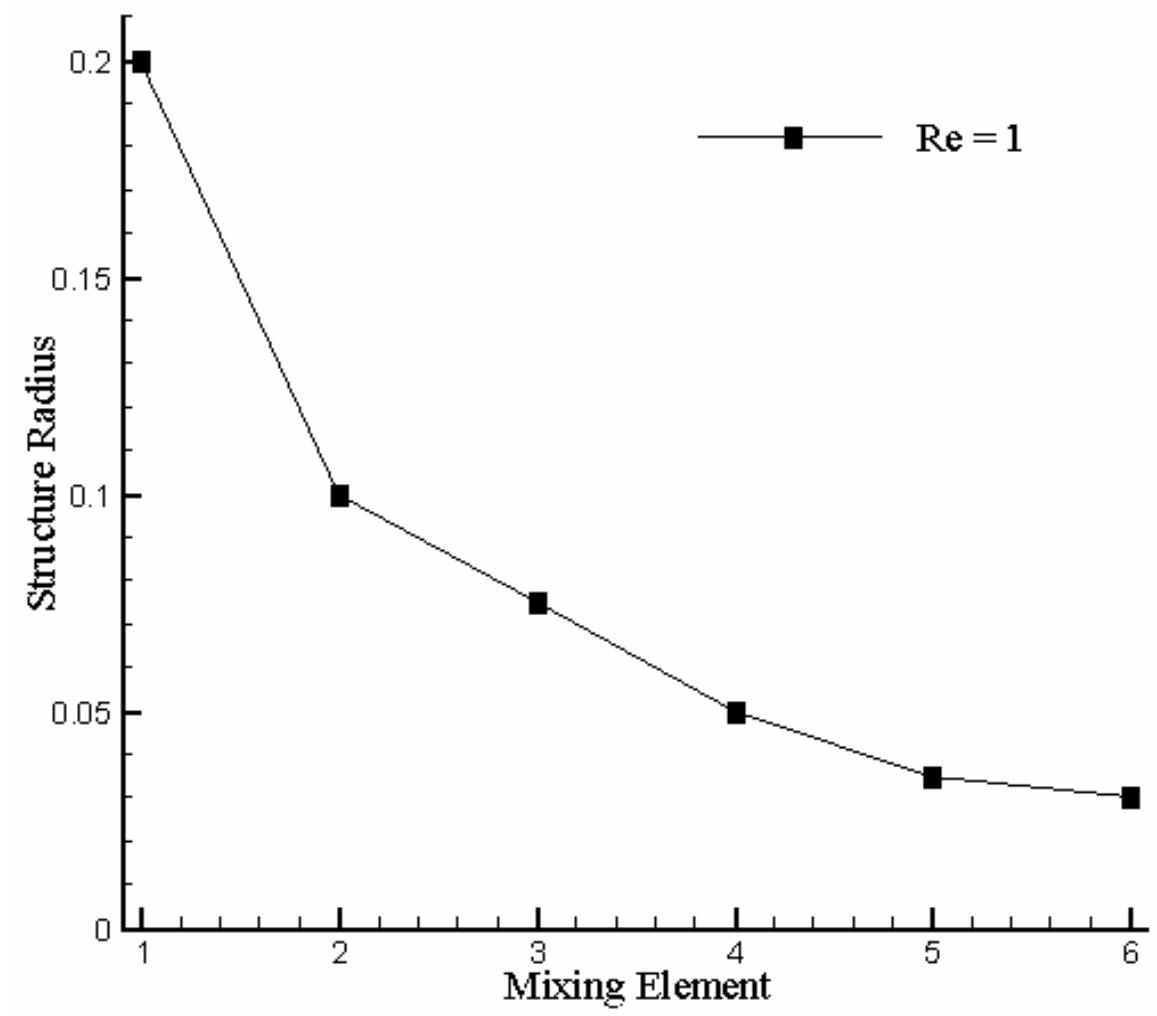

Figure 5.24: Structure radii at the end of each mixing element $(\operatorname{Re}=1)$ 


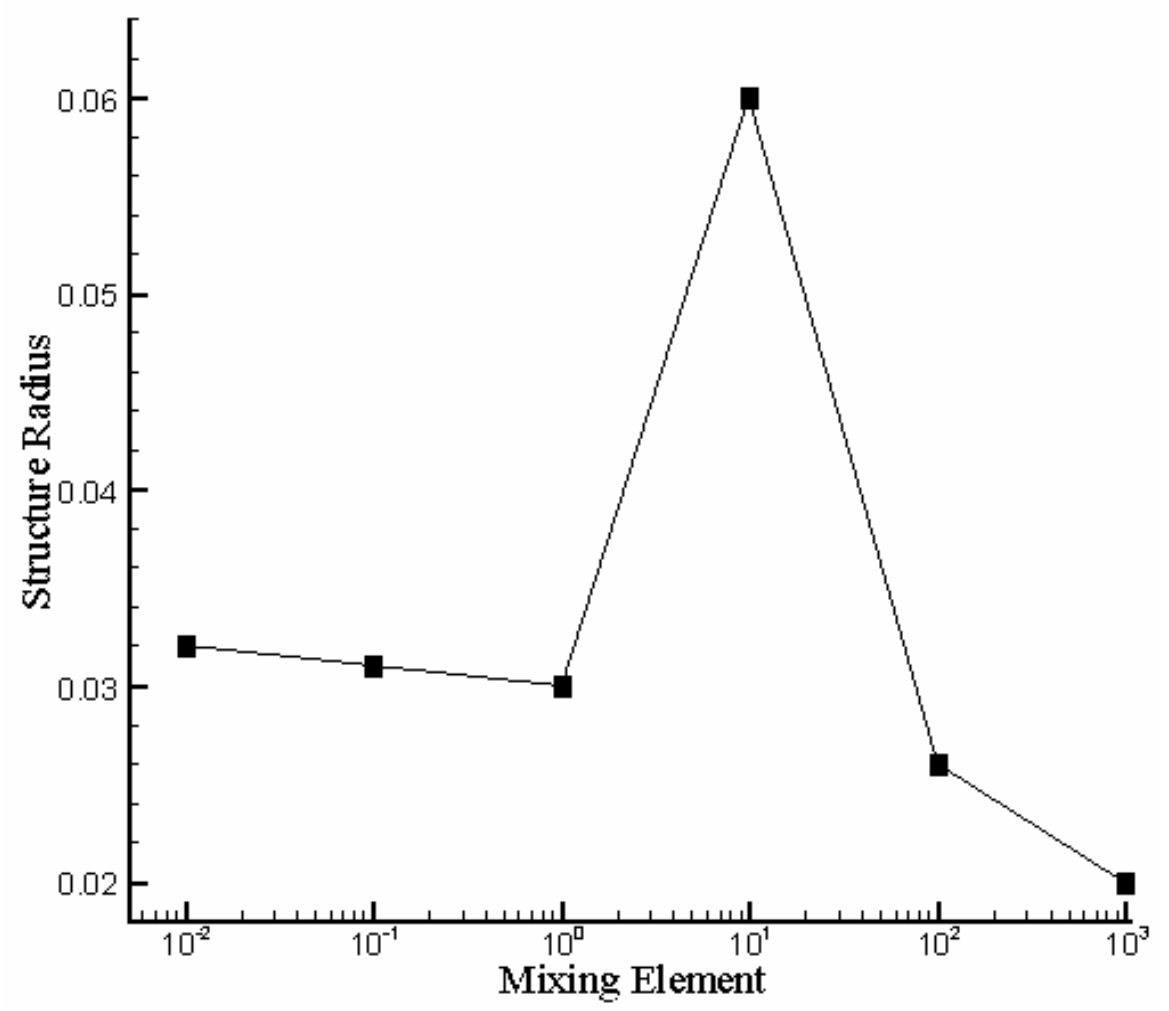

Figure 5.25: Structure radii at the end of the last mixing element 


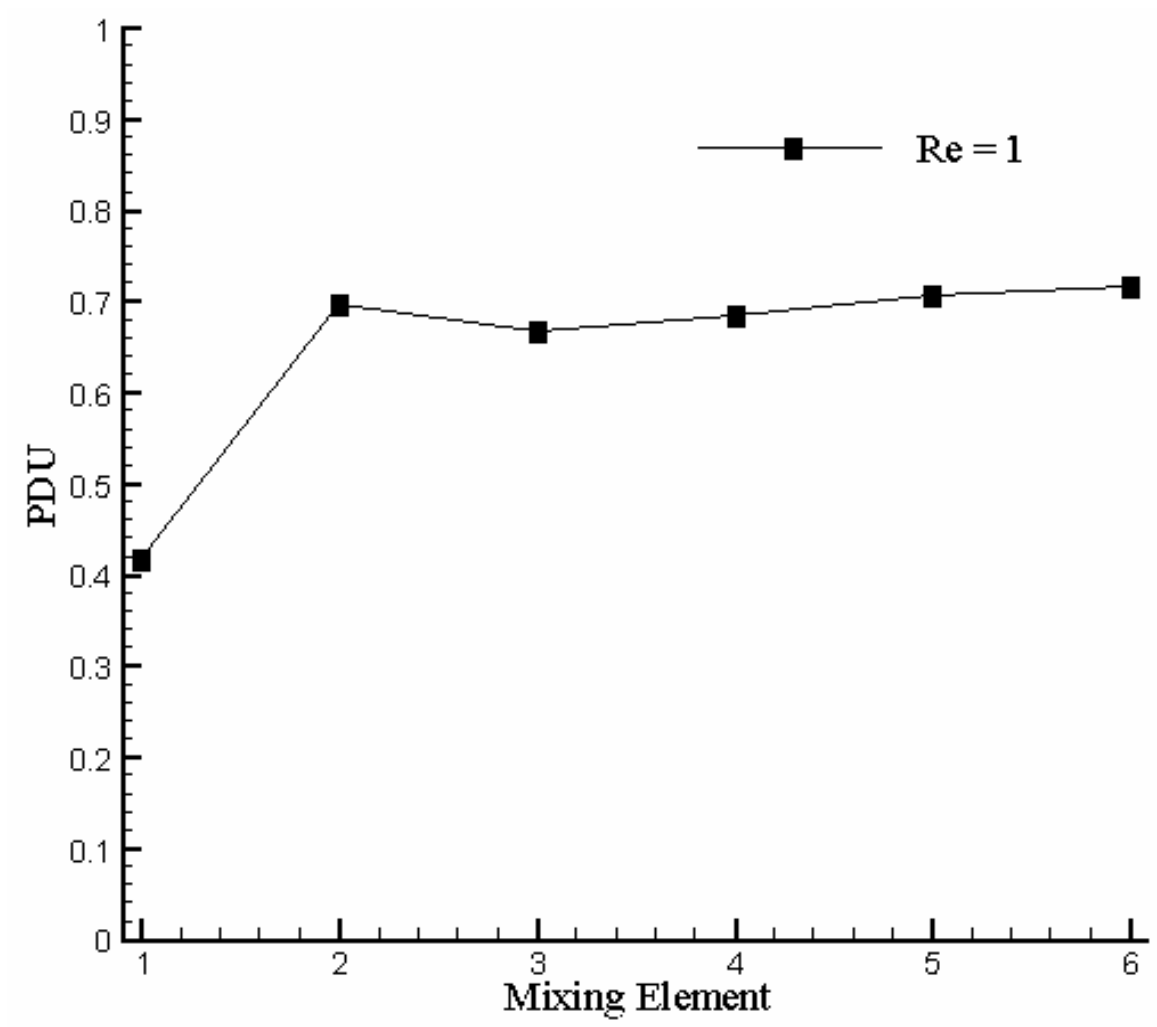

Figure 5.26: PDU values at the end of each mixing element $(\operatorname{Re}=1)$ 


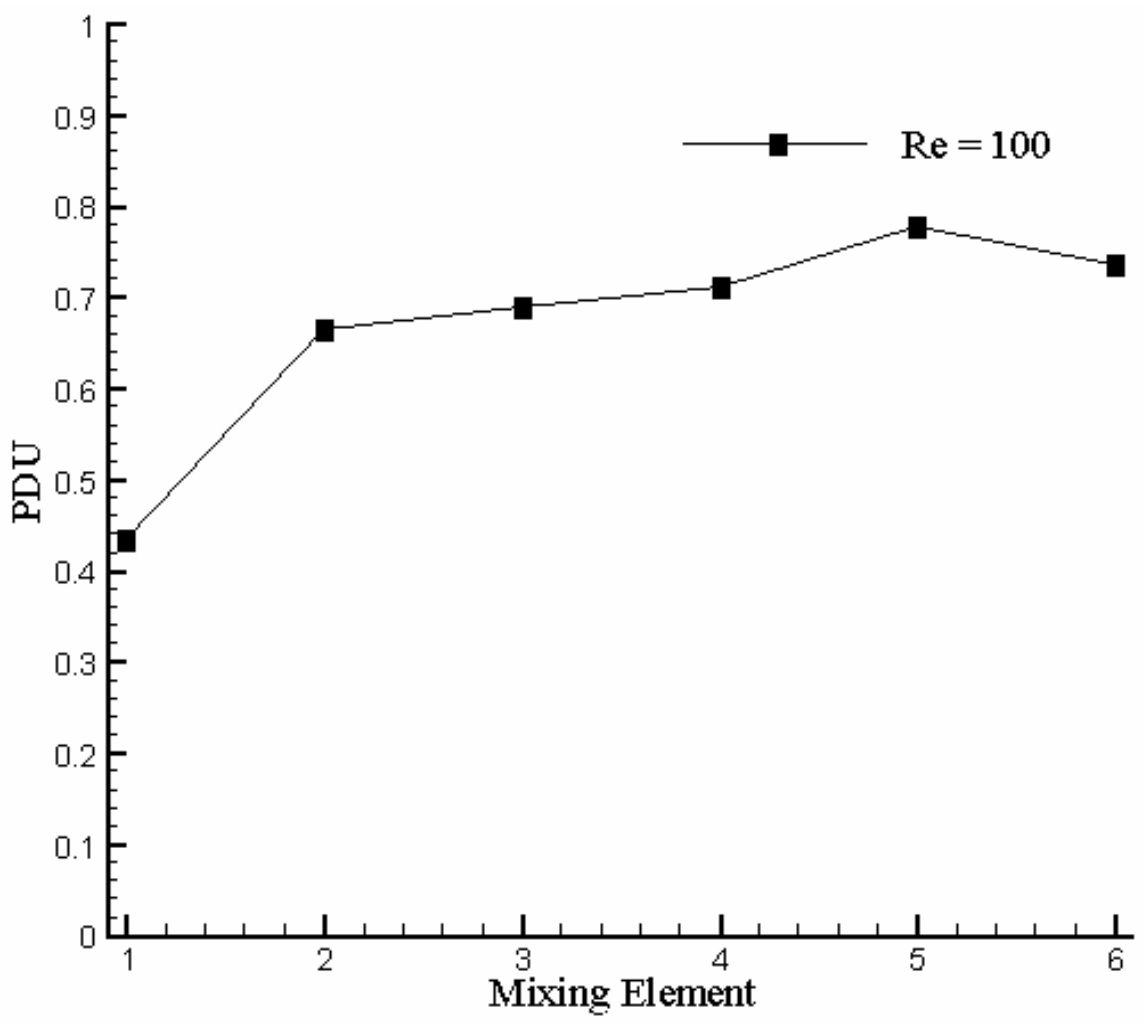

Figure 5.27: PDU values at the end of each mixing element $(\operatorname{Re}=100)$ 


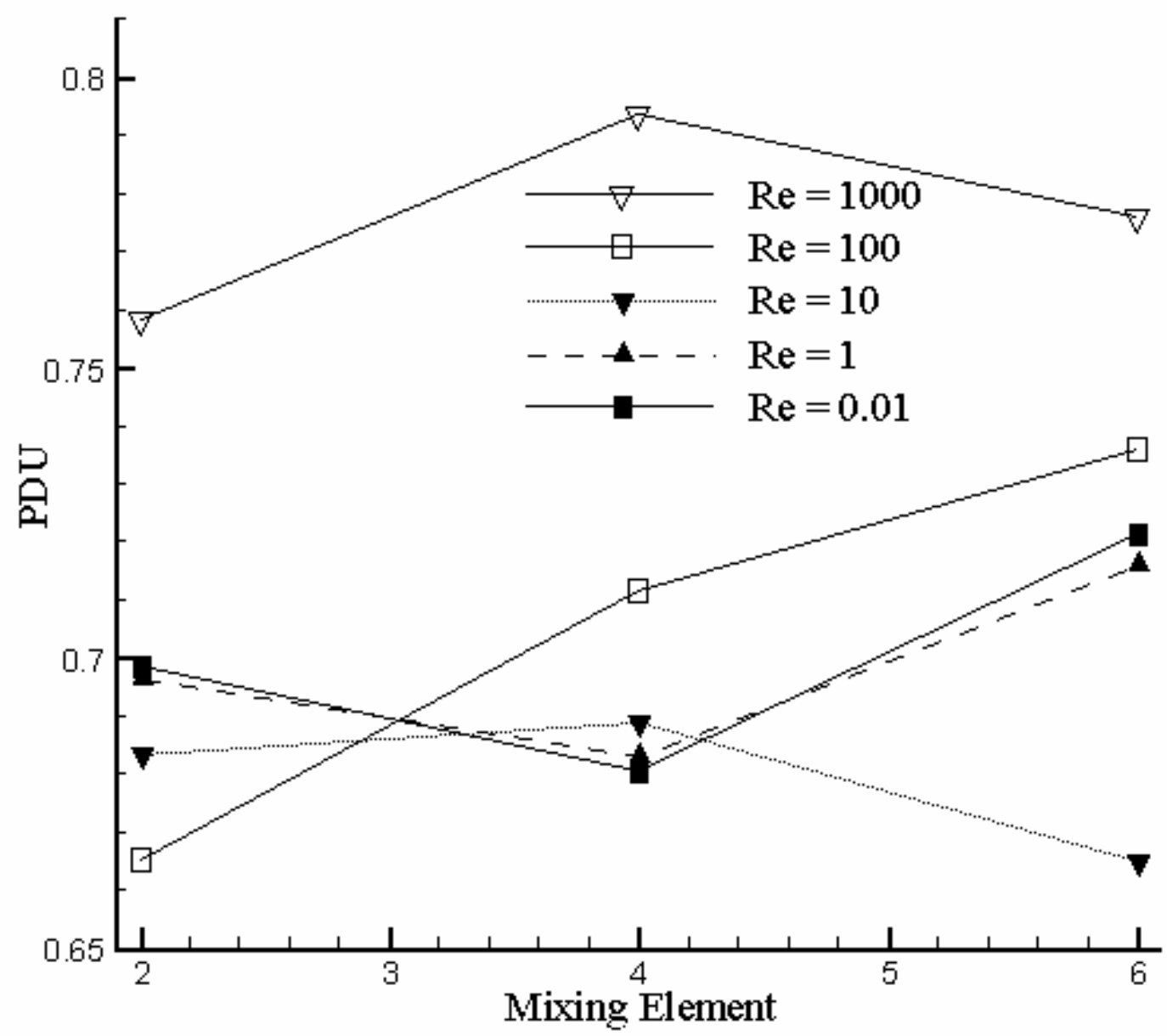

Figure 5.28: PDU values at the end of even numbered mixing elements 


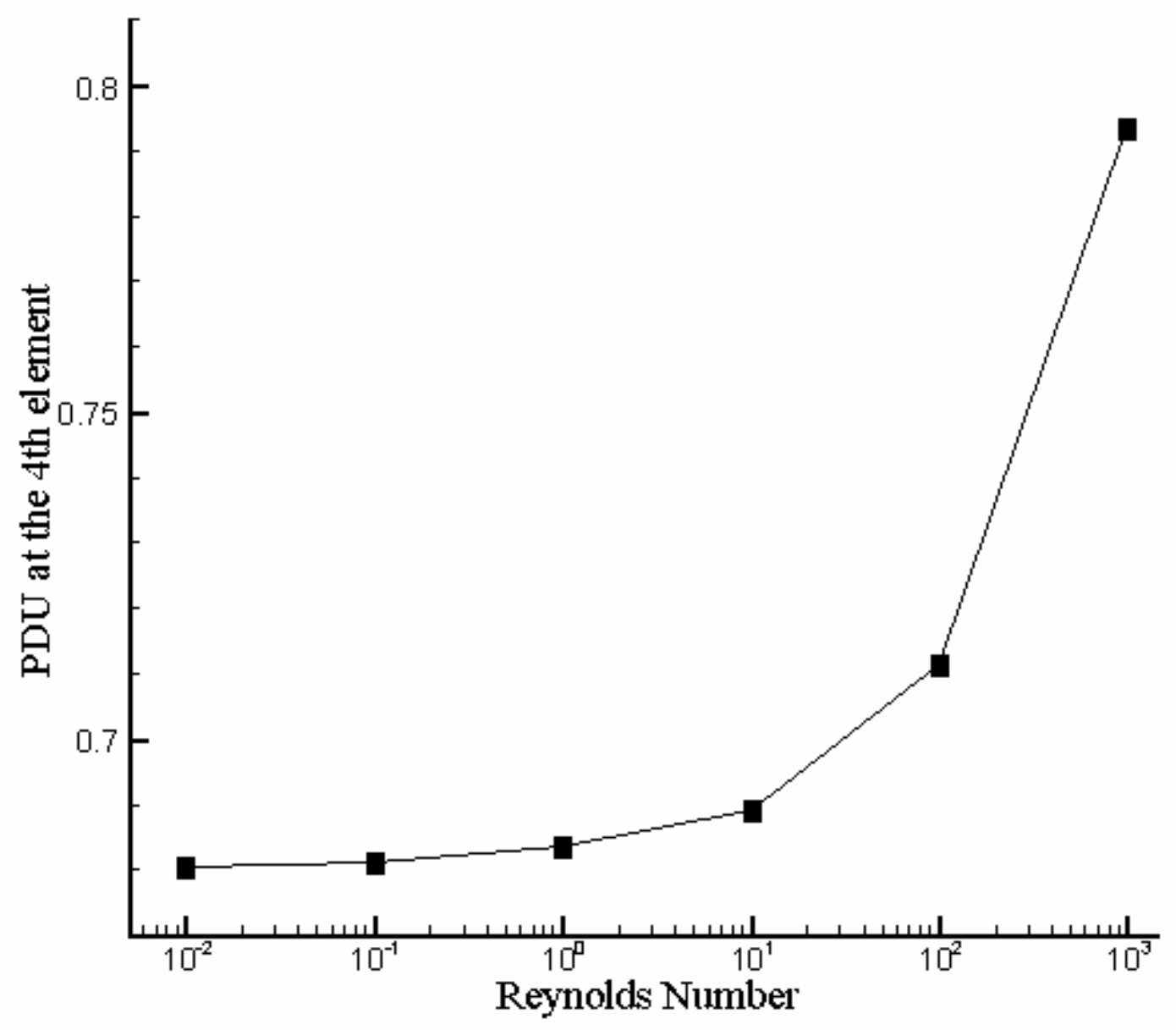

Figure 5.29: PDU values at the fourth mixing element 


\section{CHAPTER SIX}

\section{MIXING STUDY OF NON-NEWTONIAN FLUIDS IN A HELICAL STATIC MIXER}

Use of static mixers to process non-Newtonian fluids is quite common. Data on the pressure drop of non-Newtonian fluids in static mixers and the degree of mixing of materials through the mixer are very useful in the design and engineering application of these tools. Due to the industrial importance of static mixers, many studies have been undertaken in attempts to characterize their performance. Pressure drop across a static mixer was studied experimentally for Newtonian and non-Newtonian fluids [3,5-7,32], since it is essential in order to correctly size the extruder or pump, feeding the static mixer. A set of consistency and power law indices, for a CMC solution flow inside different static mixers, is given by Kale et al. [6,7]; however Xu et al. [32], use specific values for the rheological properties of a CMC solution. Moreover, heat transfer [8], mass transfer [9], and also drop size distribution [12] in static mixers have been studied experimentally.

In this chapter, the flow velocities, pressure drops, etc. are calculated for various flow rates, using the Carreau and the power law models for non-Newtonian fluids. The numerical predictions by these two models are compared to existing experimental data. 
The performance of a helical static mixer under non-Newtonian fluid working conditions is studied in detail. Also, a comparison of the mixer performance for both Newtonian and non-Newtonian fluids is presented in this chapter.

\subsection{Non-Newtonian Material: Carboxymethyl Cellulose Solution in Water}

A wide range of working fluids in industrial mixers are non-Newtonian, mostly pseudo-plastic, fluids. The non-Newtonian fluid studied in this research is carboxymethyl cellulose (CMC) in water, which is a member of the pseudo-plastic fluids group, characterized by a progressively decreasing slope or shear stress versus shear rate. These

fluids are termed shear thinning since the viscosity decreases with increasing velocity gradient. CMC is used primarily in foods, drugs and cosmetics as a viscosifier, emulsion stabilizer, and thickener to improve texture. The main applications of a technical grade of $\mathrm{CMC}$ are in textile warp sizing and in paper processing. CMC is also used in detergents as an antiredeposition (the process of preventing redeposition) agent, adhesives, latex paints and polishes. In the past, several studies have been performed to investigate CMC properties or have reported making use of CMC properties, e.g., [76-79].

Numerical techniques are applied to quantitatively evaluate the mixing performance of a static mixer for shear thinning non-Newtonian fluids. The range of modified Reynolds numbers is from $\operatorname{Re}^{\prime}=0.1$ to $\operatorname{Re}^{\prime}=1,000$, where $\operatorname{Re}^{\prime}$ is given by: 


$$
\operatorname{Re}^{\prime}=\frac{\rho U^{2-n} d^{n}}{\frac{\kappa}{8}\left(\frac{6 n+2}{n}\right)^{n}}
$$

For an incompressible Newtonian fluid flow in a given geometry, the flow Reynolds number is proportional to the mass flow rate. For a non-Newtonian flow in a given geometry, there is no linear relationship between the mass flow rate and the flow Reynolds number. Table 6.1 presents the mass flow rates for different Reynolds numbers and different CMC concentrations used in this study.

For Newtonian fluids, viscosity is independent of the strain rate of the flow. For nonNewtonian fluids, viscosity becomes a function of the strain rate. In this study, two models are considered: the power law model, and the Carreau model. CMC is wellknown as a power law material; therefore, the power law model was considered to model the flow of $\mathrm{CMC}$ solutions in a static mixer. In order to predict the mixer performance accurately, CMC fluids are modeled also by the Carreau law model for the shear stress, which provides an accurate representation of fluid behavior.

The power law model for the shear stress uses the following equation for the viscosity:

$$
\mu=\kappa e^{\frac{T}{T_{0}}} \dot{\gamma}^{n-1}
$$


The Carreau model attempts to describe a wide range of fluids by the establishment of a curve-fit to piece together functions for both Newtonian and shear-thinning nonNewtonian laws. The Carreau model uses the following equation for the viscosity:

$$
\mu=\mu_{\infty}+\left(\mu_{0}-\mu_{\infty}\right)\left[1+\left(\lambda e^{\frac{T}{T_{0}}} \dot{\gamma}\right)^{2}\right]^{\frac{n-1}{2}}
$$

Values of $\mu_{0}, \lambda, \kappa$, and $n$ used in Eqs. (6.2) and (6.3) for this study can be found in Table 6.2; these values are based on the data given in [76-78]. $\mu_{\infty}$ is assumed to be zero. The reference temperature $\left(T_{0}\right)$ is $25^{\circ} \mathrm{C}$, which is also the working temperature in this study. It may worth mentioning that the temperature has a significant effect on the rheological parame ters of CMC [81].

The power law model predicts infinite viscosity when the shear rate goes to zero. The difference between values of viscosity predicted by the power law and the Carreau models is significant when the shear rate is small. Figure 6.1 shows the difference between the viscosities predicted by these two models for different shear rate values. This difference is non-dimensionalized by dividing by the viscosity value predicted by the Carreau model.

It should be noted that the CMC solution is a pseudo-plastic or shear thinning fluid. Most of non-Newtonian fluids in industry fall into the shear thinning fluids group. Other 
kinds of non-Newtonian fluids (such as dilatant or Bingham fluids) have different rheological properties, which may lead to different static mixer performance.

\subsection{Numerical Predictions}

In order to simulate laminar flow of a Newtonian fluid 1,043,294 computational cells were used. For non-Newtonian material laminar flow simulation, 1,691,199 computational cells are needed to obtain a numerically converged prediction of the flowfield. A detailed mesh convergence study, similar to the one presented in detail in Chapter 5, indicated that the computational mesh employed in the present study is sufficiently refined to provide good numerical resolution.

For the Carreau model, comparison with certain experimental values of the pressure drop across the mixer measured for the standard element twist angle shows very good agreement over a range of flow conditions $0.1=\operatorname{Re}^{\prime}=1,000$; the maximum error occurs for a CMC concentration level of 50 when $\mathrm{Re}^{\prime}=1$, for which the experimental pressure drop is $1.2 \mathrm{~Pa}$ and the predicted pressure drop is $1.54 \mathrm{~Pa}$. For other cases the numerical pressure is in good agreement with the experimental data. However, the power law model fails to predict the pressure drop across the mixer for some flow conditions. This point will be discussed in more detail in the subsequent section.

Using the numerical method described above, the flow in a six-element static mixer has been analyzed for a number of different flow conditions. In each case the initial conditions are set so that the axial velocity $\left(u_{1}\right)$ is equal to bulk velocity and $u_{2}=u_{3}=0$. 
The number of iterations required to obtain a converged solution was found to be a function of the flow regime. For a mesh containing 1,691,199 cells, from 830 iterations (for creeping flow) to 2320 iterations (for laminar flow) were necessary (with convergence defined as achieving a residual less than $10^{-5}$, defined as the L2 norm) for the power law model. The number of iterations needed for the Carreau model ranged from 830 to 2280 . The effect of model, used for the viscosity non-Newtonian fluid, on the number of iterations needed to obtain a converged solution is not significant. Also, for flows with the same Reynolds number and different levels of CMC concentration, the number of iterations needed to obtain a converged solution is almost the same as above.

\subsubsection{Flowfield Parameters}

Figures 6.2-6.4 show the pressure drop through a six-element static mixer predicted by the Carreau model and the existing experimental data for CMC concentrations of 50, 500, and $5000 \mathrm{ppm}$. The pressure drop values across the mixer for different Reynolds numbers predicted by the Carreau model are in excellent agreement with existing experimental data. It should be mentioned that the $\mathrm{CMC}$ concentration level has a significant impact on the pressure drop values across the static mixer. For the same Reynolds number, the pressure drop for the case of a CMC concentration level of 500 $\mathrm{ppm}$ is of the order of $10^{-2}$ of the pressure drop for the case of a concentration level of $5000 \mathrm{ppm}$; and for the case of a concentration level of $50 \mathrm{ppm}$, it is of the order of $10^{-1}$ of the pressure drop, as that a concentration level of $500 \mathrm{ppm}$. 
The power law model's results do not agree well with the experimental data, especially when the Reynolds number is small. Also it was found that for a prescribed Reynolds number, the pressure drop predicted by the power law model is less accurate when the CMC concentration is higher, as it can be seen in Table 6.3. The values shown by the $\Delta P^{*}$ symbol are the predicted pressure drop by the power law model, divided by the pressure drop predicted by the Carreau model.

The following are results based on the Carreau model for shear-thinning fluids.

For a CMC solution in water with a concentration level 5,000 ppm, the planar crosssection velocity fields at the end of even numbered mixing elements are presented in Figures 6.5-6.8 for $\operatorname{Re}^{\prime}=1,10,100$ and 1,000. To obtain clear images, the length scale of the product of the velocity vector and the Reynolds number in each case is set to a constant. For other concentration levels (50 ppm and $500 \mathrm{ppm}$ ) of CMC in water, the velocity domain is the same as the velocity domain for a concentration level of 5,000 ppm, when the Reynolds number is the same. Different flow patterns for creeping and laminar flows can be seen. It may be observed that after the second element, four velocity zones are created. These zones combine together and create two velocity zones, as the flow passes through the mixer. For higher Reynolds number flows, regions of strong cross-sectional vortices are distinguishable.

The vorticity magnitudes at the end of the even numbered mixing elements are presented in Table 6.4 for a CMC solution (with a concentration level of 5,000). As may be expected, the mean and the maximum vorticity magnitudes are increased by increasing the Reynolds number. Therefore, a higher degree of mixing is expected within the same 
flow cross-section when the Reynolds number is high. Also, it is observed that the vorticity magnitude is overall smaller at the end of the last mixing element compared to the vorticity magnitude at the other elements; the flow after the last mixing element gradually merges to that of the flow in a pipe with no mixer. For the last mixing element, the mean vorticity magnitude is much smaller than the maximum vorticity magnitude. This suggests that flow around the internal mixing elements has a higher mixing potential; although by increasing the Reynolds number the contrast between the mean and the maximum values of vorticity at the last mixing element and the other mixing elements is less pronounced.

It is important to compare the performance of the static mixer with Newtonian fluids against that with shear thinning non-Newtonian fluids. Compared to the results obtained for Newtonian fluids, in Chapter 5, it can be seen that the velocity patterns and the separated flow zones are similar for flows in the pipe, with the same Reynolds number, in both cases.

Comparison of the vorticity contours shows that the order of magnitude of the vorticity for a $\mathrm{CMC}$ solution is $10^{2}$ times the order of magnitude of the vorticity for a Newtonian fluid (water); however both follow nearly the same pattern.

Examination of the pressure drops across the helical static mixer using Newtonian and non-Newtonian fluids shows a significant increase in pressure drop for the case of a non-Newtonian fluid, as shown in Table 6.7. The values denoted within the $\left(\Delta P_{\text {Newtonian }} / \Delta P_{P_{\text {seudo-plastic }}}\right)$ column represent the pressure drop for a Newtonian fluid (water), divided by the pressure drop for a CMC solution in water. Adding CMC to water 
leads to more energy required to maintain the flow through a static mixer inside of a pipe or channel. For a Reynolds number of one, the pressure drop of water flow across the sixelement helical static mixer is about $6 \%$ of the pressure drop across mixer when the working fluid is a $\mathrm{CMC}$ solution with a concentration level of $50 \mathrm{ppm}$. By increasing the Reynolds number the difference between pressure drops for these two working fluids is decreased; for a Reynolds number of one thousand, the pressure drop of water flow across the six-element helical static mixer is about $35 \%$ of the pressure drop across mixer when the working fluid is a CMC solution with a concentration level of $50 \mathrm{ppm}$. Also, by increasing the concentration level of $\mathrm{CMC}$ from 50 to $500 \mathrm{ppm}$ the pressure drop ratio decreases one order of magnitude; and by increasing the concentration level of CMC from 500 to $5000 \mathrm{ppm}$ the pressure drop ratio decreases two orders of magnitude. Hence, increasing the concentration level of $\mathrm{CMC}$ solution increases the required energy significantly.

By comparing the vorticity magnitudes between those obtained for a Newtonian fluid flow in mixer, Chapter 5, and those for a non-Newtonian fluid flow in mixer, it is found that the order of magnitude of the vorticity for a Newtonian fluid is $10^{-2}$ times the order of magnitude of the vorticity for a CMC solution; however, both follow nearly the same pattern.

\subsubsection{Particle Trajectories}

The trajectories of particles injected into the mixer from the top half of the flow inlet have been calculated. For the case of CMC concentrations of 50,500, and 5,000 ppm, 
using the Carreau model, the plots of the particle positions at the end of the second, the fourth, and the sixth elements, respectively from top to bottom (shown in Figs. 6.9-6.23) illustrate the redistribution of the released particles via the combined effects of flow division and reversal, resulting in stretching and folding of the observed structures. Islands of separated flow are distinguishable after the flow passes the second mixing element. Increasing the Reynolds number results in these few large islands breaking into several smaller islands. The islands, observed after the second elements in low Reynolds number flows, divide into narrow line shaped regions when flow passes the fourth element, and can still be recognized after the sixth element for lower Reynolds number. Again, increasing the Reynolds number results in the separated areas breaking and therefore enhances the mixing of fluid particles in the same cross-section.

Comparing the obtained particle distributions for the cases of $\operatorname{Re}^{\prime}=0.1$ and $\operatorname{Re}^{\prime}=1$ shows no difference between the patterns, leading to the conclusion that for very bw Reynolds numbers, fluid mixing is virtually the same. The particles distribution pattern is still similar to those patterns when the Reynolds number increases to 10 ; however, the patterns observed for $\operatorname{Re}^{\prime}=100$ and $\mathrm{Re}^{\prime}=1000$ are different. Spiral motion and areas of swirling flow are distinguishable for higher Reynolds numbers. For low Reynolds numbers the particle distribution pattern is anti-symmetric. This pattern can be observed after the second mixing element for higher Reynolds numbers; however, as the flow passes through the mixer the particle distribution changes into a more symmetric pattern for higher Reynolds numbers. 


\subsubsection{G-value}

The G-value can be determined for a helical static mixer, using the following expression:

$$
\mathrm{G} \text { - value }=\frac{1}{2}\left(\frac{\pi D}{V_{m}}\right)^{1 / 2}\left(\frac{\operatorname{Re} \Delta p}{\rho}\right)^{1 / 2}
$$

For the six-element mixer studied here, using the geometrical parameters, given in Table 1.1, Eq. (6.4) can be simplified to the following:

$$
\mathrm{G} \text { - value }=105.75\left(\frac{\operatorname{Re} \Delta p}{\rho}\right)^{1 / 2}
$$

Figures 6.24-6.26 show the calculated G-values for different CMC concentration levels. All three logarithmic diagrams are slightly non-linear, suggesting that for a given material, the G-value in the helical static mixer is proportional to the flow Reynolds number. G-value increases significantly when the CMC concentration level is increased, indicating that more energy is required to maintain the flow in the pipe. 


\subsubsection{Residence Time Distribution (RTD)}

To study the RTD for different CMC concentrations in the static mixer, the distribution function for CMC concentration levels of 50,500, and $5000 \mathrm{ppm}$ were calculated and are shown in Figs. 6.27-6.41 for $\operatorname{Re}^{\prime}=0.1,1,10,100$, and 1000, respectively. The residence time distribution for $\mathrm{CMC}$ solutions of different concentration levels is very similar for a specific Reynolds number, especially in low Reynolds number flows. For higher Reynolds numbers, values of the distribution function at a given $t^{*}$ is slightly different for different concentration levels. For $\mathrm{Re}^{\prime}=$ 0.1 , one mode of $f\left(t^{*}\right)$ is distinguished for all concentration levels, which starts at about $t^{*}=0.4$ and ends at about $t^{*}=3.3$. For $t^{*}$ values less than two, the difference between distribution functions for different concentration levels is indistinguishable. For $\mathrm{Re}^{\prime}=1$, two modes of $f\left(t^{*}\right)$ are distinguishable for all concentration levels; the first mode starts at about $t^{*}=0.5$ and ends at about $t^{*}=2.0$ to 2.4. As can be seen for $\operatorname{Re}^{\prime}=1, f\left(t^{*}\right)$ is almost the same for different solution concentration levels, when $t^{*}$ is less than one. Above a $t^{*}$ value of one, the obtained patterns for $f\left(t^{*}\right)$ are less similar, although $f\left(t^{*}\right)$ values have the same order of magnitude. For $\mathrm{Re}^{\prime}=10$, although the distribution function follows the same pattern for all different levels of concentration, the difference between $f\left(t^{*}\right)$ for a CMC solution with a concentration level of 5,000 ppm and a CMC solution with concentration levels of 50 and $500 \mathrm{ppm}$ is significant when $t^{*}$ is greater than two. The maximum $t^{*}$ is also different for different concentration levels. For a CMC solution with a concentration level of 5,000 ppm, the maximum value of $t^{*}$ is about 3.7; 
for the other two cases it is about 4.4, showing that fluid elements of a CMC solution with high concentration have a more similar residence history in a helical static mixer. By increasing the Reynolds number to 100 and 1,000, the differences of distribution function patterns and values are more pronounced.

For a CMC solution with a concentration level of $50 \mathrm{ppm}$, it is found that by increasing the Reynolds number from 0.1 to 1 , a second mode of $f\left(t^{*}\right)$ is created. The second mode is more visible when the Reynolds number is higher. It also is observed that by increasing the Reynolds number, the maximum value of $t^{*}$ decreases; however for $\mathrm{Re}^{\prime}=10$ this not the case. The maximum value of $t^{*}$ is 3.34 for $\mathrm{Re}^{\prime}=0.1$ and decreases to 3.29 when Reynolds number is one. The maximum $t^{*}$ is 4.35 when $\operatorname{Re}^{\prime}=10$ and it decreases to 2.40 when the Reynolds numbers increases to 100 and it is 2.14 , when the Reynolds number reaches 1000 . The same pattern is observed for CMC concentration levels of 500 and $5000 \mathrm{ppm}$. It suggests that for mixing processes that chemical reactions are a major part of process, flows with a Reynolds number around 10 can decrease the quality of the process.

For a specific working fluid, comparing residence time distribution for flow Reynolds numbers of $0.1 \leq \operatorname{Re}^{\prime} \leq 1000$ shows that for the cases of $\operatorname{Re}^{\prime}=100$, and 1,000 the distribution function has a superior pattern. For example, for a solution with a concentration level of 5,000 and flow Reynolds number of 100, the distribution function is more symmetric and for this case the maximum $t^{*}$ is 2.4 , while for $\mathrm{Re}^{\prime}=1$ and 10 , the maximum $t^{*}$ is about 3.2 . 
The standard deviations of the distribution function for different flow conditions are shown in Table 6.8. For the case of an specific Reynolds number, the difference between values of standard deviations for flows with different levels of CMC concentration are very close, indicating that the distribution function is not influenced by the rheological properties of the fluid. The standard deviation is a function of the flow Reynolds number. The standard deviation increases slightly, when Reynolds number increases from 1 to 10 . By increasing the Reynolds number from 10 to 100 and again from 100 to 1,000, standard deviation decreases. For the case of a CMC concentration level of 5,000 ppm, as can be seen, the values of the standard deviation of the distribution function for various Reynolds numbers are significantly different. The standard deviation of the distribution function for the case of $\mathrm{Re}^{\prime}=100$ is $22 \%$ smaller than the standard deviation of the distribution function for the case of $\operatorname{Re}^{\prime}=1$ and it is $27 \%$ smaller than the standard deviation of the distribution function for the case of $\mathrm{Re}^{\prime}=10$. For the case of $\mathrm{Re}^{\prime}=$ 1,000 , the standard deviation of the distribution function is about $70 \%$ of the standard deviation of the distribution function for the case of $\operatorname{Re}^{\prime}=100$. This indicates that the static mixer has a better residence time distribution for the flows with a higher Reynolds number.

\subsubsection{Structure Radius}

By observing Figs. 6.9-6.23, it is recognized that the non-Newtonian fluid concentration level has no impact on the fluid elements redistribution in the flowfield. Therefore, structure radius in a given flow cross-section is not a function of the fluid 
concentration. Figure 6.42 presents structure radii at different flow cross-sections for a CMC solution with concentration level of $5000 \mathrm{ppm}$ for $\mathrm{Re}^{\prime}=1$. At the flow inlet $r_{s}=0.5$, and at the end of the first mixing element $r_{s} \approx 0.2$. At the end of the second element $r_{s} \approx 0.1$. As the flow passes through the mixer, the structure radius decreases and the rate of decrease of the structure radius also decreases. Structure radius has virtually the same pattern and virtually the same magnitudes for both Newtonian and Non-Newtonian working fluids, when the flow Reynolds number is one. Figure 6.43 presents structure radii at the end of the last mixing element for $\mathrm{Re}^{\prime}=0.1,1,10,100$, and 1000 . The general pattern is that by increasing the Reynolds number, the structure radius should decrease. However, for $\mathrm{Re}=10$, the radius structure at the last mixing element is higher compared to the radius structure at the same flow cross-section, when $\operatorname{Re}=1$ or less. It suggests that working fluids are less mixed at this Reynolds number, compare to the case of $\mathrm{Re}=1$. Comparing the results obtained for Newtonian working fluid, it is observed that the structure radius follows the same pattern, however, for Reynolds number equal or greater than ten, the structure radius values are slightly different. For a flow Reynolds number of $10, r_{s}$ is about $20 \%$ larger for the nonNewtonian fluid, which suggests that the helical static mixer has a better performance when the working fluid is Newtonian; although, the overall performance of a helical static mixer is low at a Reynolds number of 10 for both Newtonian and non-Newtonian fluids. 


\subsubsection{Particles Distribution Uniformity (PDU)}

The PDU values after the second, the fourth, and the sixth mixing elements are shown in Figs. 6.44-6.46, for different CMC concentration levels and flow Reynolds numbers. These values are calculated based on the velocity fields predicted by the Carreau model. The PDU values for creeping flow conditions are very close and almost the same as those shown in the figures for the case of $\mathrm{Re}^{\prime}=1$. Increasing the Reynolds number increases the PDU values. A significant increase in the PDU values can be seen when the Reynolds number increases to 100; by increasing the Reynolds number from 100 to 1,000, again the PDU values increase at the second mixing element, and also at the other mixing elements. For low Reynolds number flows, the PDU increases at the last mixing element, however, for higher Reynolds number flows, the PDU drops at the end of the helical static mixer. It can be explained by the fact that the flow enters the pipe with no mixer after this point and laminar flow in an empty pipe boundary conditions are applied to the flow, tending to the reduction of mixing process.

As might be expected, the PDU values for different CMC concentrations (50, 500 and 5,000 ppm) are very close. The largest difference between the PDU values happens for $\operatorname{Re}^{\prime}=1,000$, between CMC solutions with concentration levels of 50 and $5000 \mathrm{ppm}$, which is about $4 \%$. The second largest difference occurs for $\mathrm{Re}^{\prime}=100$, between $\mathrm{CMC}$ solutions with concentration levels of 50 and $500 \mathrm{ppm}$, which is about $2 \%$. For other cases, differences between the PDU values for the same Reynolds numbers and different CMC concentration levels amount to about one percent or less. 
To quantify the performance of the helical static mixer, studied here, the obtained PDU values at the sixth mixing element for a Newtonian fluid and for a CMC solution are compared in Table 6.9. It is observed that, for the low Reynolds numbers flow condition, the PDU values are nearly the same for both Newtonian and shear thinning, non-Newtonian, fluids. The PDU value increases more for a CMC solution by increasing the Reynolds number to 100. For a Reynolds number of 1,000, the helical static mixer manifests slightly higher performance when the working fluid is Newtonian. Also, as can be seen, the static mixer performance is low at a Reynolds number of 10 for both Newtonian and shear thinning, non-Newtonian, fluids.

\subsubsection{Helical Static Mixer Efficiency}

The efficiency of an industrial helical static mixer can be defined based on the required energy that must be supplied to flow the materials through the system and the degree of the achieved mixing of those materials. The PDU value at the last mixing element is used to determine the mixer efficiency. Table 6.10 presents the six-element static mixer efficiency for different CMC solutions and different Reynolds numbers. The CMC solution concentration level has almost no impact on the mixer efficiency. Mixer efficiency is almost the same for different concentration levels, when the flow Reynolds number is the same. Figure 6.48 compares helical static mixer efficiency with a Newtonian working fluid to its efficiency with a Non-Newtonian working fluid. Helical static mixer has a higher efficiency for Non-Newtonian fluids. For low Reynolds number flows, when the working fluid is non-Newtonian, static mixer efficiency is more than two 
times of the mixer efficiency, when the working fluid is Newtonian. For higher Reynolds numbers, the superiority of the static mixer with non-Newtonian fluids to the mixer with Newtonian fluids is less pronounced; for a Reynolds number of 1,000 , the helical static mixer efficiency with a non-Newtonian fluid is about $15 \%$ more than the static mixer efficiency with a Newtonian fluid. 


\begin{tabular}{|c|c|c|}
\hline $\begin{array}{c}\text { CMC } \\
\text { Conc. } \\
\text { (ppm) }\end{array}$ & $\operatorname{Re}^{\prime}$ & $\begin{array}{c}\text { Mass flow } \\
(\mathrm{Kg} / \mathrm{s})\end{array}$ \\
\hline 50 & 0.1 & 0.0000029 \\
\hline 50 & 1 & 0.0000198 \\
\hline 50 & 10 & 0.0001374 \\
\hline 50 & 100 & 0.0009544 \\
\hline 50 & 1000 & 0.0066276 \\
\hline 500 & 0.1 & 0.0000105 \\
\hline 500 & 1 & 0.0000705 \\
\hline 500 & 10 & 0.0004726 \\
\hline 500 & 100 & 0.0031693 \\
\hline 500 & 1000 & 0.0212526 \\
\hline 5000 & 0.1 & 0.0000848 \\
\hline 5000 & 1 & 0.0005685 \\
\hline 5000 & 10 & 0.0038123 \\
\hline 5000 & 100 & 0.0255641 \\
\hline 5000 & 1000 & 0.1714259 \\
\hline
\end{tabular}

Table 6.1: Mass flow rates for different Reynolds number and different CMC concentrations 


\begin{tabular}{|c|c|c|c|c|}
\hline $\begin{array}{c}\text { Concentration } \\
(\mathrm{ppm})\end{array}$ & $n$ & $\begin{array}{c}\kappa \\
\left(\mathrm{Pa}_{\mathrm{s}} \mathrm{s}^{n-1}\right)\end{array}$ & $\begin{array}{c}\mu_{0} \\
(\mathrm{~Pa} . \mathrm{s})\end{array}$ & $\begin{array}{c}\lambda \\
(s)\end{array}$ \\
\hline 50 & 0.8118 & 0.00561 & 0.0042 & 0.214 \\
\hline 500 & 0.7900 & 0.02629 & 0.0190 & 0.213 \\
\hline 5000 & 0.7900 & 0.32839 & 0.3100 & 0.760 \\
\hline
\end{tabular}

Table 6.2: Rheological properties of CMC solutions 


\begin{tabular}{|c|c|c|}
\hline $\mathrm{Re}^{\prime}$ & $\begin{array}{c}\text { CMC } \\
\text { Conc. } \\
(\mathrm{ppm})\end{array}$ & $\Delta P^{*}$ \\
\hline 0.1 & 50 & 1.034 \\
\hline 0.1 & 500 & 1.2025 \\
\hline 0.1 & 5000 & 2.3685 \\
\hline 1 & 50 & 1.3091 \\
\hline 1 & 500 & 1.7454 \\
\hline 1 & 5000 & 3.2989 \\
\hline 10 & 50 & 1.7410 \\
\hline 10 & 500 & 2.3601 \\
\hline 10 & 5000 & 3.0099 \\
\hline 100 & 50 & 1.2291 \\
\hline 100 & 500 & 1.2797 \\
\hline 100 & 5000 & 1.2818 \\
\hline 1000 & 50 & 1.1046 \\
\hline 1000 & 500 & 1.1058 \\
\hline 1000 & 5000 & 1.1058 \\
\hline
\end{tabular}

Table 6.3: Pressure drop prediction for different Reynolds number and different CMC concentrations 


\begin{tabular}{|c|c|c|c|c|c|c|}
\cline { 2 - 7 } \multicolumn{1}{c|}{} & \multicolumn{3}{c|}{ Mean vorticity } & \multicolumn{3}{c|}{ Maximum vorticity } \\
\multicolumn{1}{c|}{$1 / s$} & \multicolumn{3}{c|}{$1 / s$} \\
\hline $\operatorname{Re}$ & $2^{\text {nd }}$ & $4^{\text {th }}$ & $6^{\text {th }}$ & $2^{\text {nd }}$ & $4^{\text {th }}$ & $6^{\text {th }}$ \\
\hline 1 & $3.74 \mathrm{E} 2$ & $3.67 \mathrm{E} 2$ & $1.51 \mathrm{E} 2$ & $1.83 \mathrm{E} 3$ & $1.77 \mathrm{E} 3$ & $2.70 \mathrm{E} 3$ \\
\hline 10 & $2.34 \mathrm{E} 3$ & $2.30 \mathrm{E} 3$ & $9.76 \mathrm{E} 2$ & $1.21 \mathrm{E} 4$ & $1.21 \mathrm{E} 4$ & $1.36 \mathrm{E} 4$ \\
\hline 100 & $3.15 \mathrm{E} 4$ & $2.94 \mathrm{E} 4$ & $1.01 \mathrm{E} 4$ & $1.35 \mathrm{E} 5$ & $1.13 \mathrm{E} 5$ & $6.91 \mathrm{E} 4$ \\
\hline 1000 & $2.72 \mathrm{E} 5$ & $2.52 \mathrm{E} 5$ & $1.18 \mathrm{E} 5$ & $1.30 \mathrm{E} 6$ & $1.23 \mathrm{E} 6$ & $9.31 \mathrm{E} 5$ \\
\hline
\end{tabular}

Table 6.4: Vorticity magnitude at 2nd, 4th, and 6th mixing element (CMC concentration level of 5000 ppm) 


\begin{tabular}{|c|c|c|c|}
\hline $\begin{array}{c}\text { Reynolds } \\
\text { number }\end{array}$ & $\begin{array}{c}\frac{\Delta P_{\text {Newtonian }}}{\Delta P_{P_{\text {seudo-plastic }}}} \\
\text { (Concentration } \\
\text { of 50 ppm) }\end{array}$ & $\begin{array}{c}\frac{\Delta P_{\text {Newtonian }}}{\Delta P_{P_{\text {seudo-plastic }}}} \\
\text { (Concentration } \\
\text { of 500 ppm) }\end{array}$ & $\begin{array}{c}\frac{\Delta P_{\text {Newtonian }}}{\Delta P_{P_{\text {seudo-plastic }}}} \\
\left(\begin{array}{c}\text { Concentration } \\
\text { of 5000 ppm) }\end{array}\right.\end{array}$ \\
\hline 1 & $6.00 \mathrm{E}-2$ & $4.98 \mathrm{E}-3$ & $7.7 \mathrm{E}-5$ \\
\hline 10 & $1.16 \mathrm{E}-1$ & $1.03 \mathrm{E}-2$ & $1.59 \mathrm{E}-4$ \\
\hline 100 & $1.70 \mathrm{E}-1$ & $1.56 \mathrm{E}-2$ & $2.41 \mathrm{E}-4$ \\
\hline 1000 & $3.49 \mathrm{E}-1$ & $3.43 \mathrm{E}-2$ & $5.30 \mathrm{E}-4$ \\
\hline
\end{tabular}

Table 6.7: Pressure drop comparison 


\begin{tabular}{|c|c|c|}
\hline $\mathrm{Re}^{\prime}$ & $\begin{array}{c}\text { CMC Conc. } \\
\text { ppm }\end{array}$ & $\begin{array}{c}\text { Standard } \\
\text { Deviation }\end{array}$ \\
\hline 0.1 & 50 & $2.4768 \mathrm{E}-002$ \\
\hline 0.1 & 500 & $2.4145 \mathrm{E}-002$ \\
\hline 0.1 & 5000 & $2.4635 \mathrm{E}-002$ \\
\hline 1 & 50 & $2.4530 \mathrm{E}-002$ \\
\hline 1 & 500 & $2.3731 \mathrm{E}-002$ \\
\hline 1 & 5000 & $2.4598 \mathrm{E}-002$ \\
\hline 10 & 50 & $2.8716 \mathrm{E}-002$ \\
\hline 10 & 500 & $2.8898 \mathrm{E}-002$ \\
\hline 10 & 5000 & $2.6218 \mathrm{E}-002$ \\
\hline 100 & 50 & $2.0723 \mathrm{E}-002$ \\
\hline 100 & 500 & $2.3236 \mathrm{E}-002$ \\
\hline 100 & 5000 & $1.9189 \mathrm{E}-002$ \\
\hline 1000 & 50 & $1.7469 \mathrm{E}-002$ \\
\hline 1000 & 500 & $1.3971 \mathrm{E}-002$ \\
\hline 1000 & 5000 & $1.3996 \mathrm{E}-002$ \\
\hline
\end{tabular}

Table 6.8: Standard deviation of the distribution function 


\begin{tabular}{|c|c|c|c|c|}
\hline $\begin{array}{c}\text { Reynolds } \\
\text { number }\end{array}$ & $\begin{array}{c}\text { PDU } \\
\text { Newtonian }\end{array}$ & $\begin{array}{c}\text { PDU } \\
\text { CMC solution } \\
50 \mathrm{ppm}\end{array}$ & $\begin{array}{c}\text { PDU } \\
\text { CMC solution } \\
500 \mathrm{ppm}\end{array}$ & $\begin{array}{c}\text { PDU } \\
\text { CMC solution } \\
5000 \mathrm{ppm}\end{array}$ \\
\hline 0.1 & 0.721 & 0.725 & 0.722 & 0.730 \\
\hline 1 & 0.716 & 0.721 & 0.722 & 0.723 \\
\hline 10 & 0.665 & 0.660 & 0.666 & 0.666 \\
\hline 100 & 0.736 & 0.793 & 0.774 & 0.775 \\
\hline 1000 & 0.776 & 0.800 & 0.770 & 0.767 \\
\hline
\end{tabular}

Table 6.9: PDU values at the sixth mixing element 


\begin{tabular}{|c|c|c|c|}
\hline $\begin{array}{c}\text { Reynolds } \\
\text { number }\end{array}$ & $\begin{array}{c}\text { Efficiency } \\
(50 \mathrm{ppm})\end{array}$ & $\begin{array}{c}\text { Efficiency } \\
(500 \mathrm{ppm})\end{array}$ & $\begin{array}{c}\text { Efficiency } \\
(5000 \mathrm{ppm})\end{array}$ \\
\hline 0.1 & 0.1011 & 0.1018 & 0.1176 \\
\hline 1 & 0.0900 & 0.0929 & 0.1167 \\
\hline 10 & 0.0952 & 0.0980 & 0.0980 \\
\hline 100 & 0.0372 & 0.0357 & 0.0366 \\
\hline 1000 & 0.0113 & 0.0106 & 0.0106 \\
Table 6.10: Helical Static Mixer Efficiency
\end{tabular}




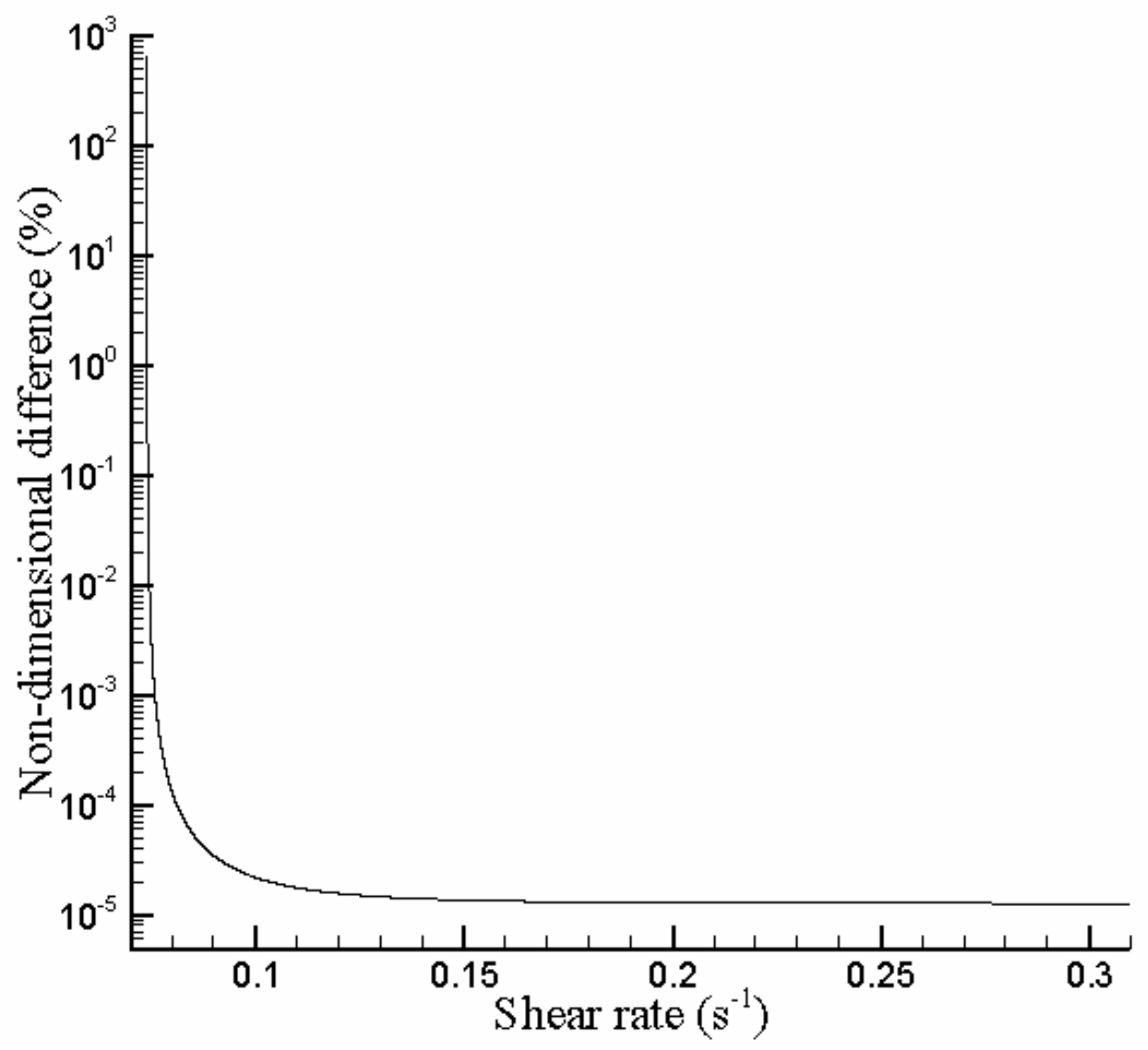

Figure 6.1: Non-dimensionalized difference between the viscosities predicted by the power law and the Carreau models 


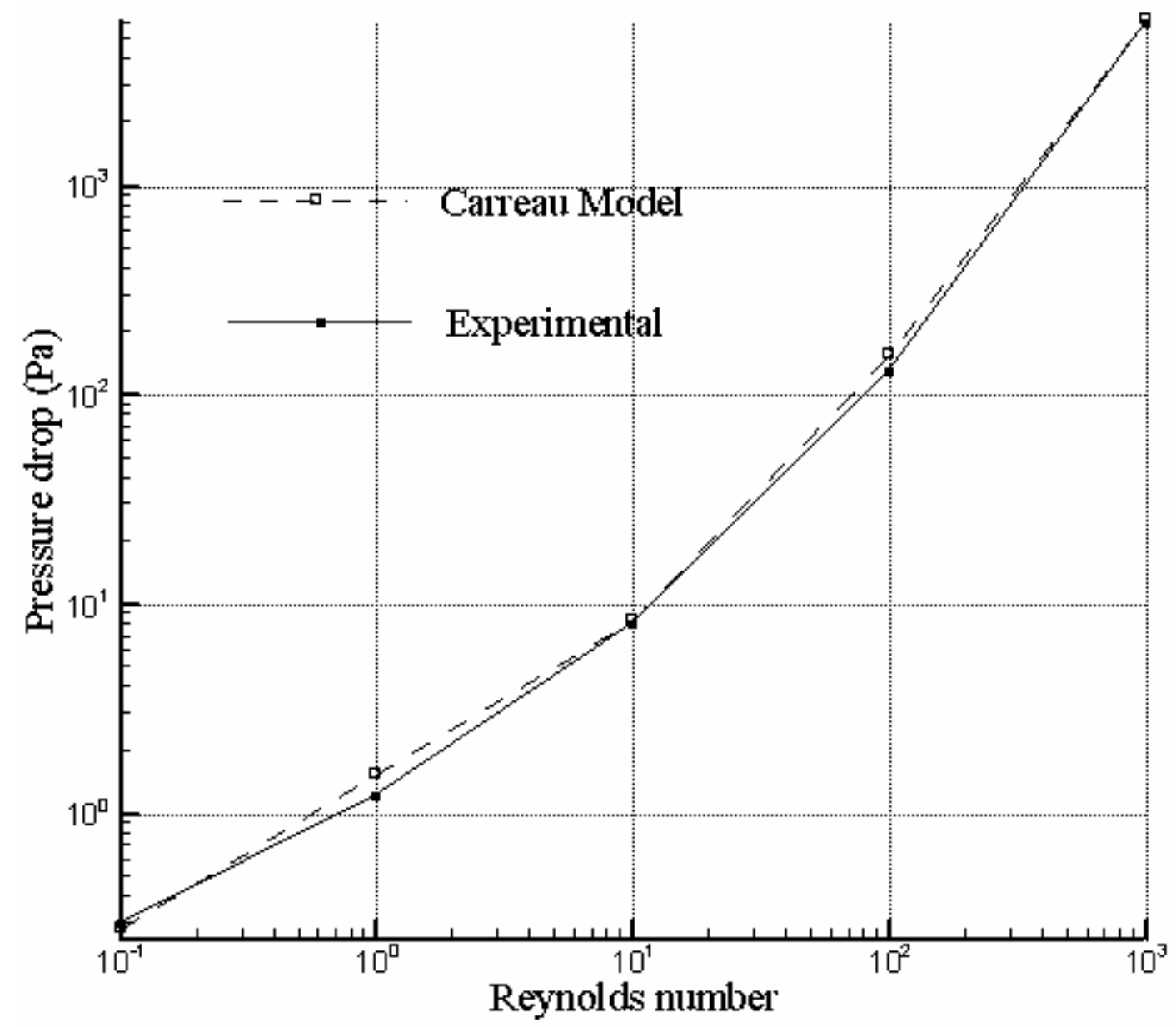

Figure 6.2: Pressure drop across a six-element helical static mixer for a CMC concentration level of $50 \mathrm{ppm}$ 


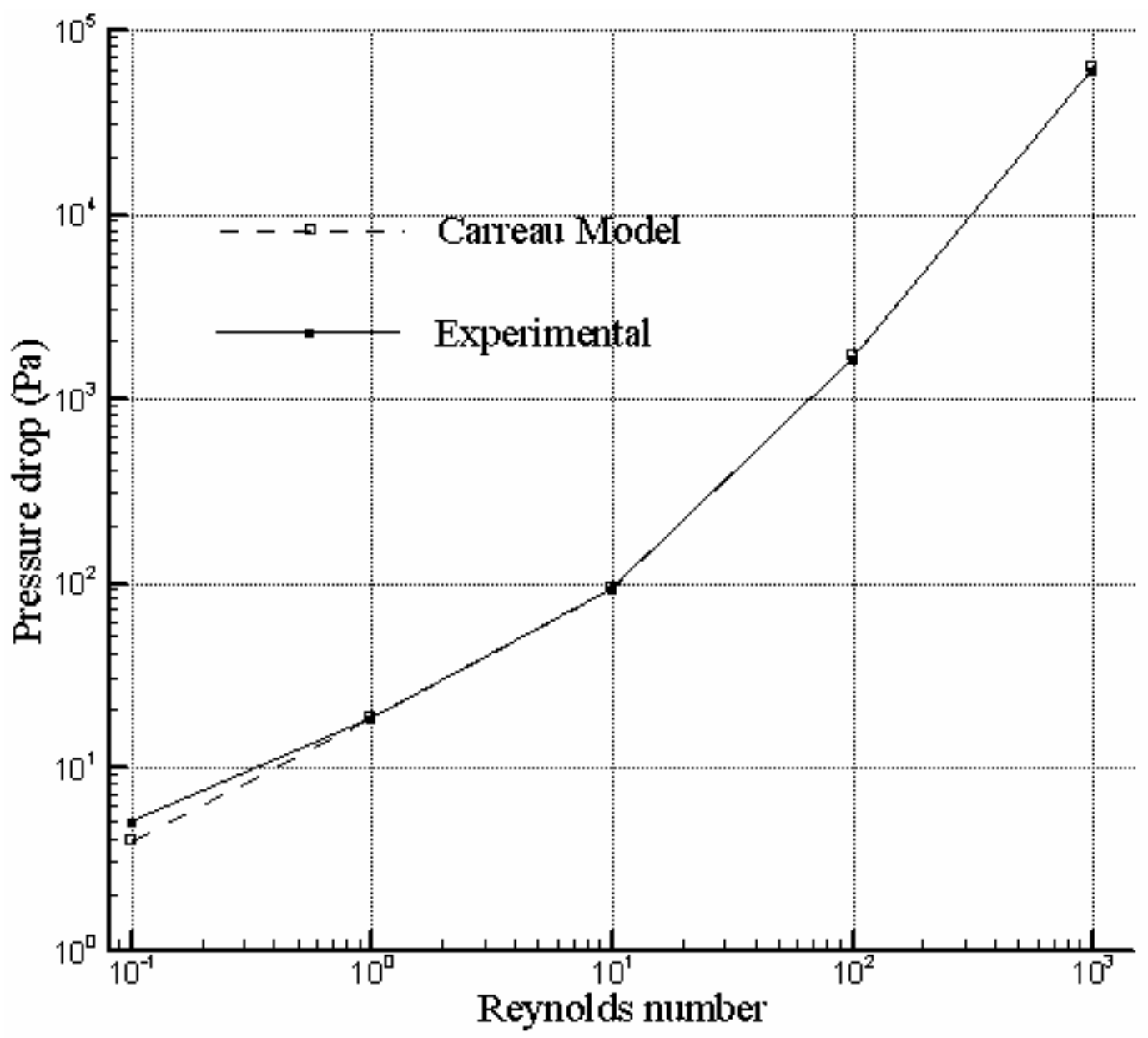

Figure 6.3: Pressure drop across a six-element helical static mixer for a CMC concentration level of $500 \mathrm{ppm}$ 


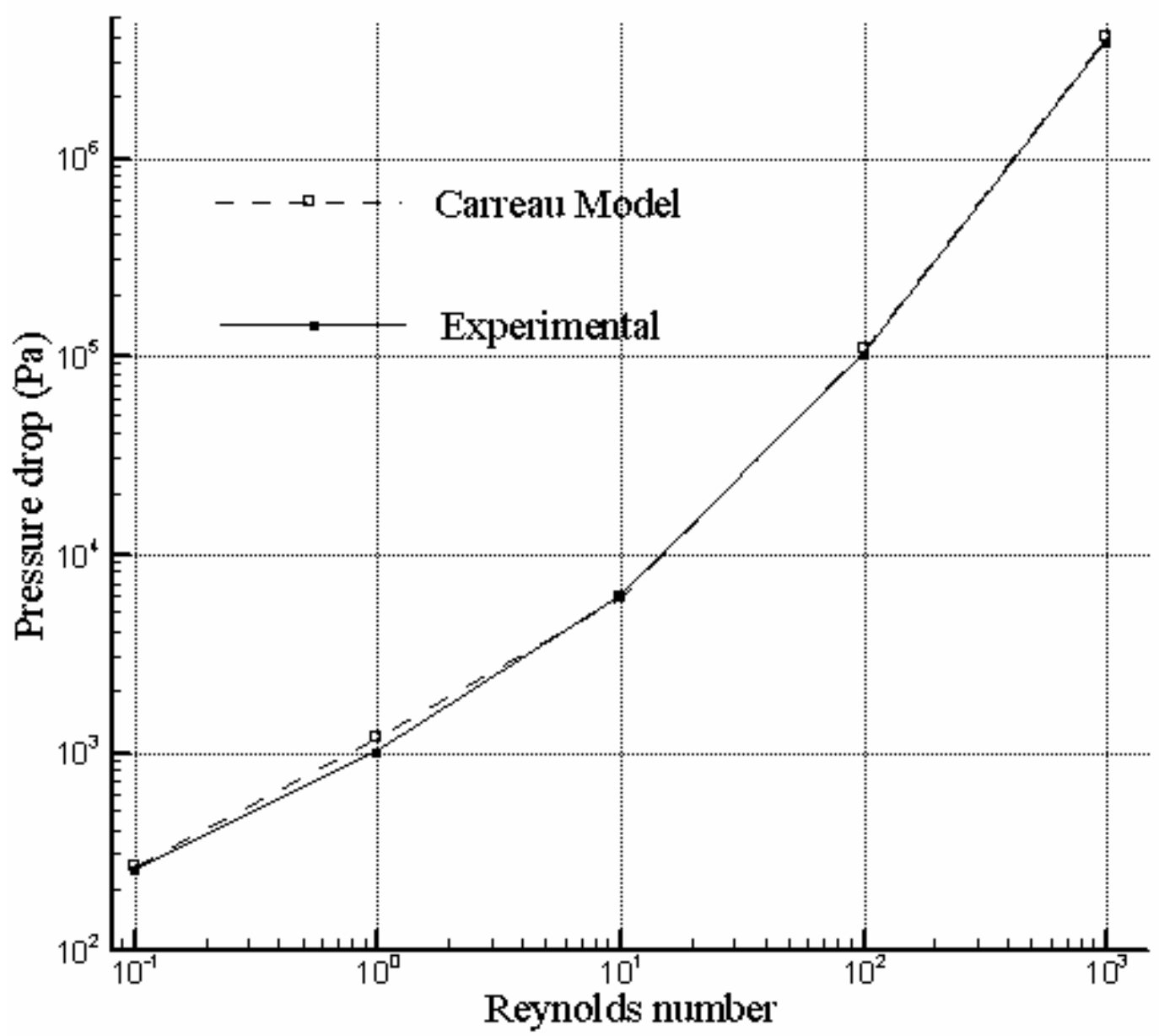

Figure 6.4: Pressure drop across a six-element helical static mixer for a CMC concentration level of 5000 ppm 

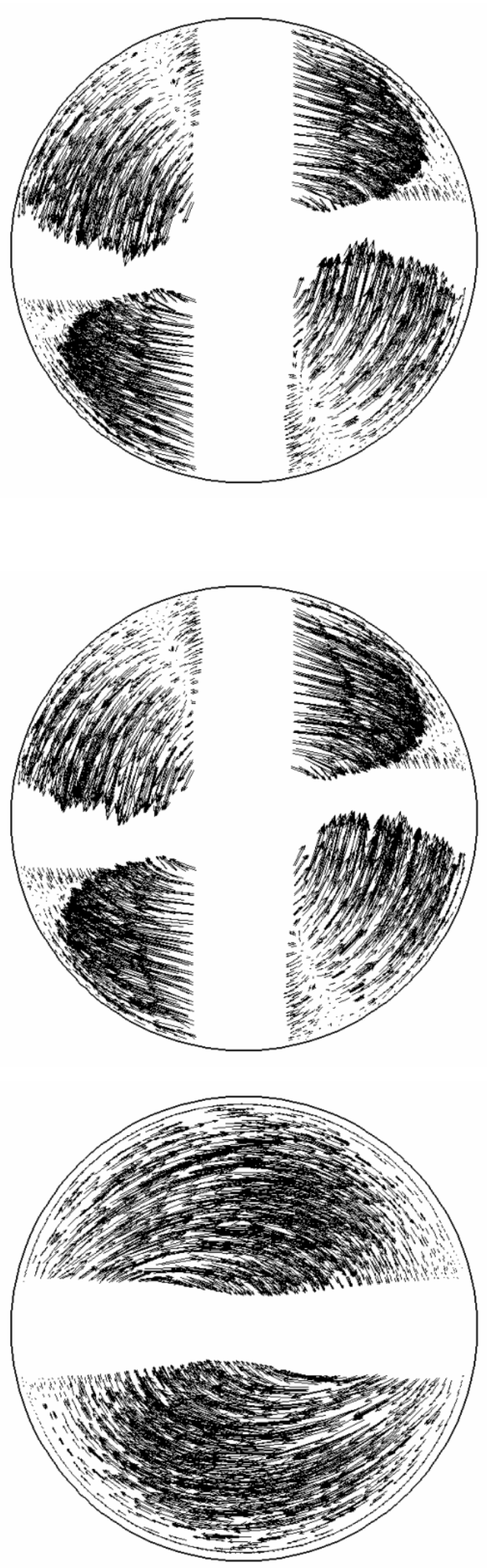

Figure 6.5: Velocity field at 2nd, 4th, and 6th mixing element ( $\operatorname{Re}^{\prime}=1$ ) 

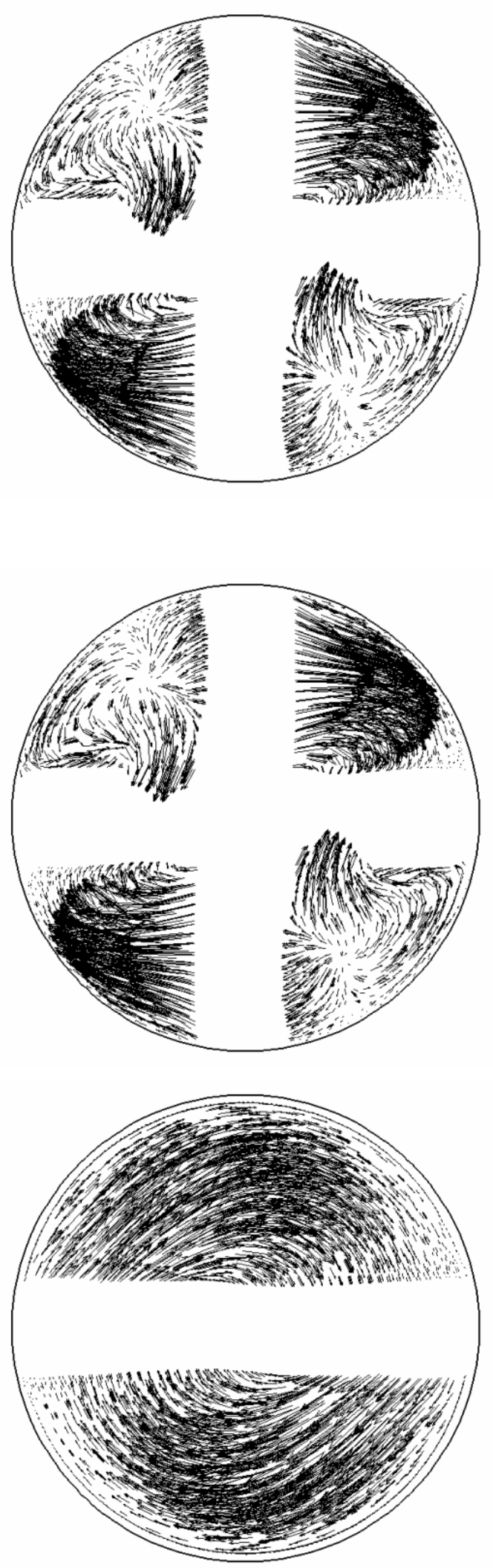

Figure 6.6: Velocity field at 2nd, 4th, and 6th mixing element $\left(\operatorname{Re}^{\prime}=10\right)$ 

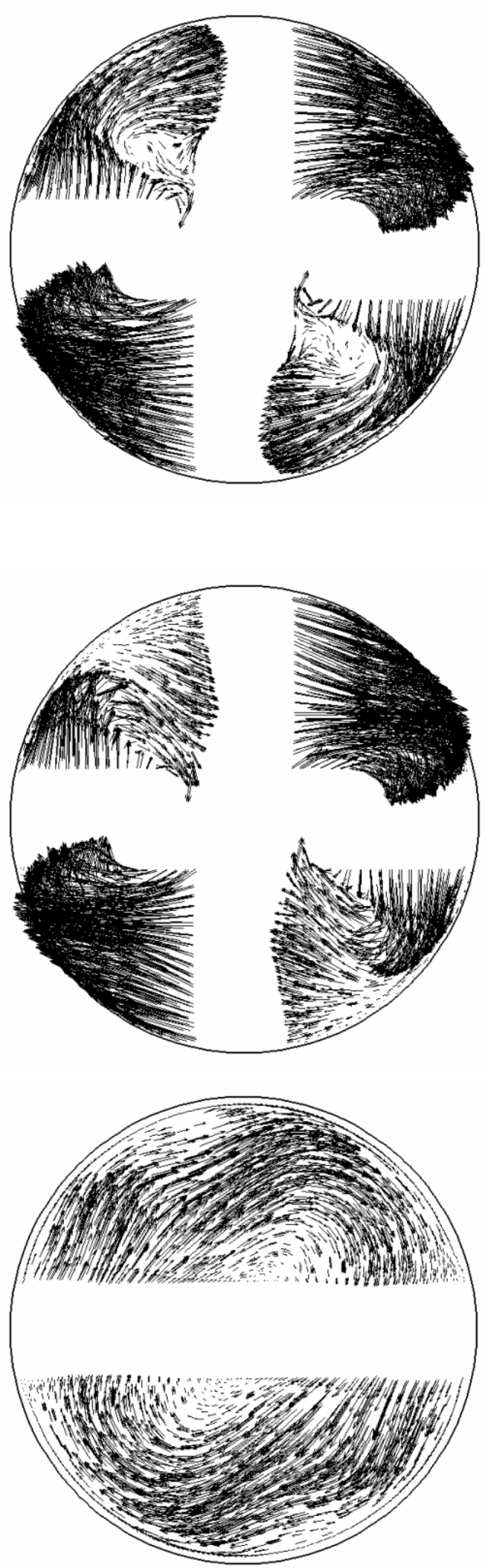

Figure 6.7: Velocity field at 2nd, 4th, and 6th mixing element for $\left(\operatorname{Re}^{\prime}=100\right)$ 

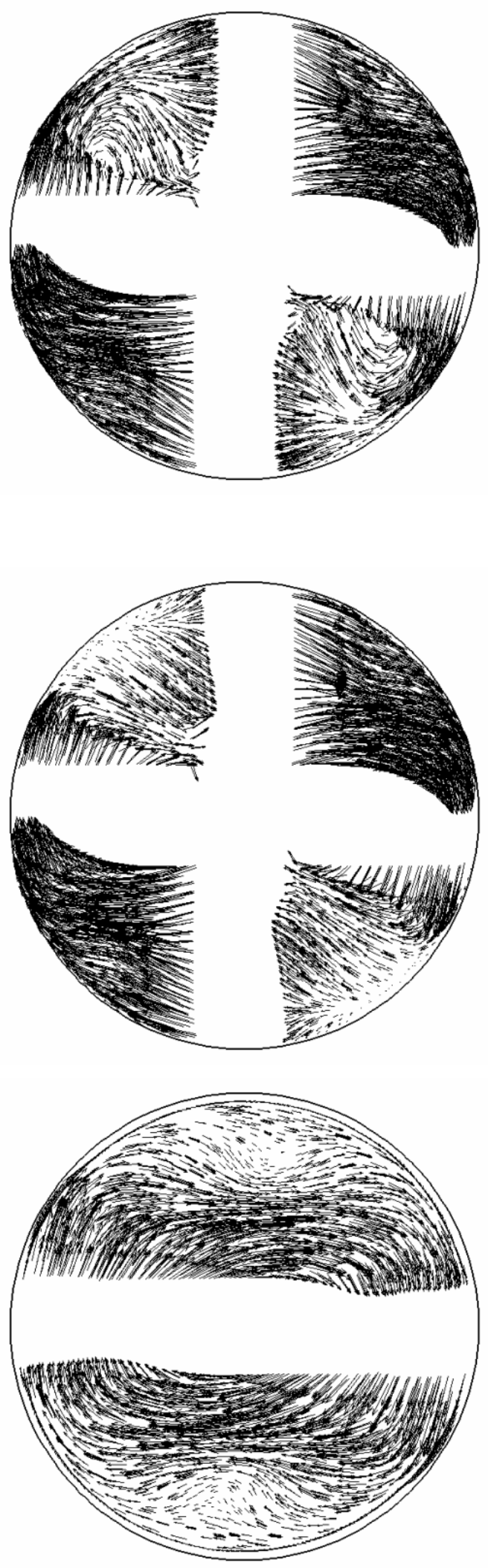

Figure 6.8: Velocity field at 2nd, 4th, and 6th mixing element $\left(\operatorname{Re}^{\prime}=1000\right)$ 

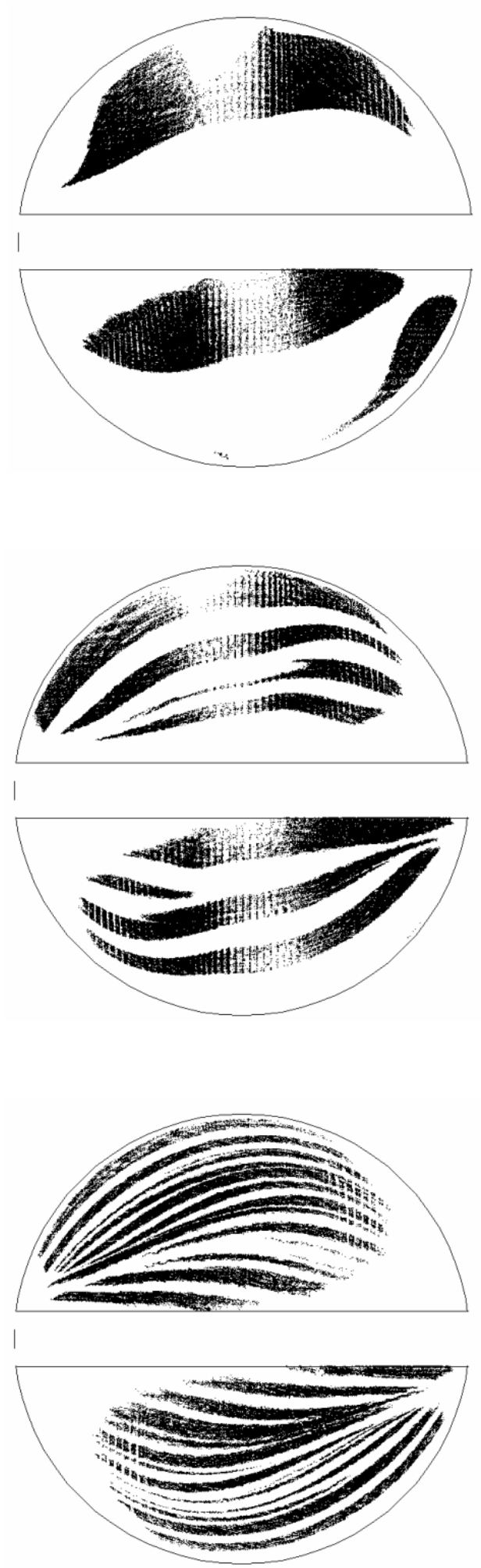

Figure 6.9: Particles locations at 2nd, 4th, and 6th mixing element for a CMC concentration level of $50 \mathrm{ppm}\left(\mathrm{Re}^{\prime}=\mathbf{0 . 1}\right)$ 

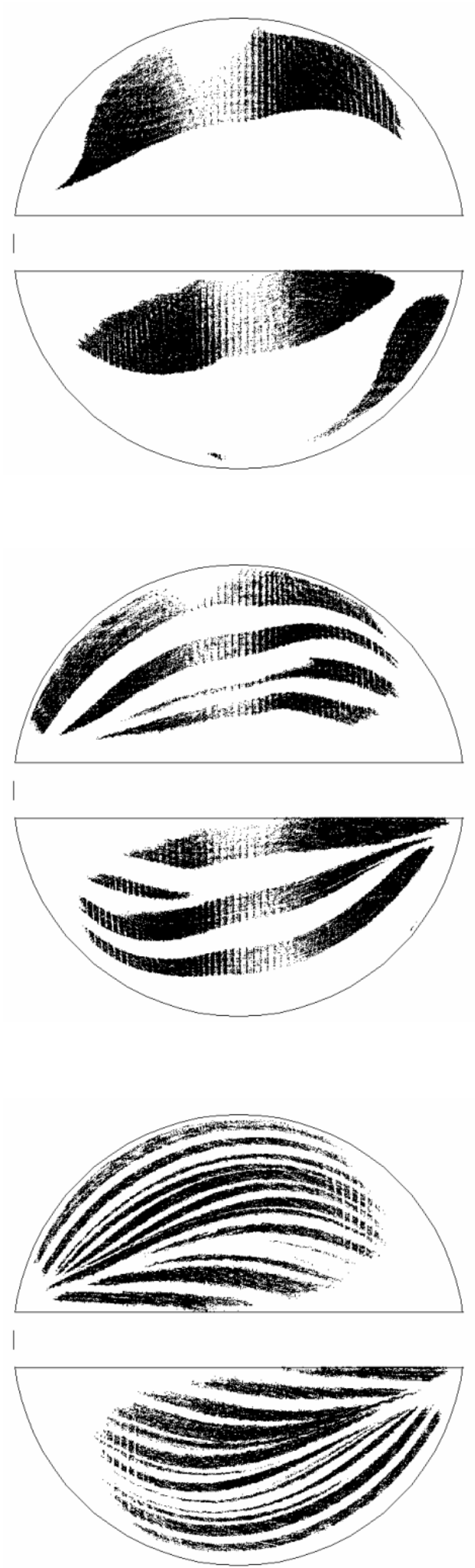

Figure 6.10: Particles locations at 2nd, 4th, and 6th mixing element for a CMC concentration level of $50 \mathrm{ppm}\left(\operatorname{Re}^{\prime}=1\right)$ 

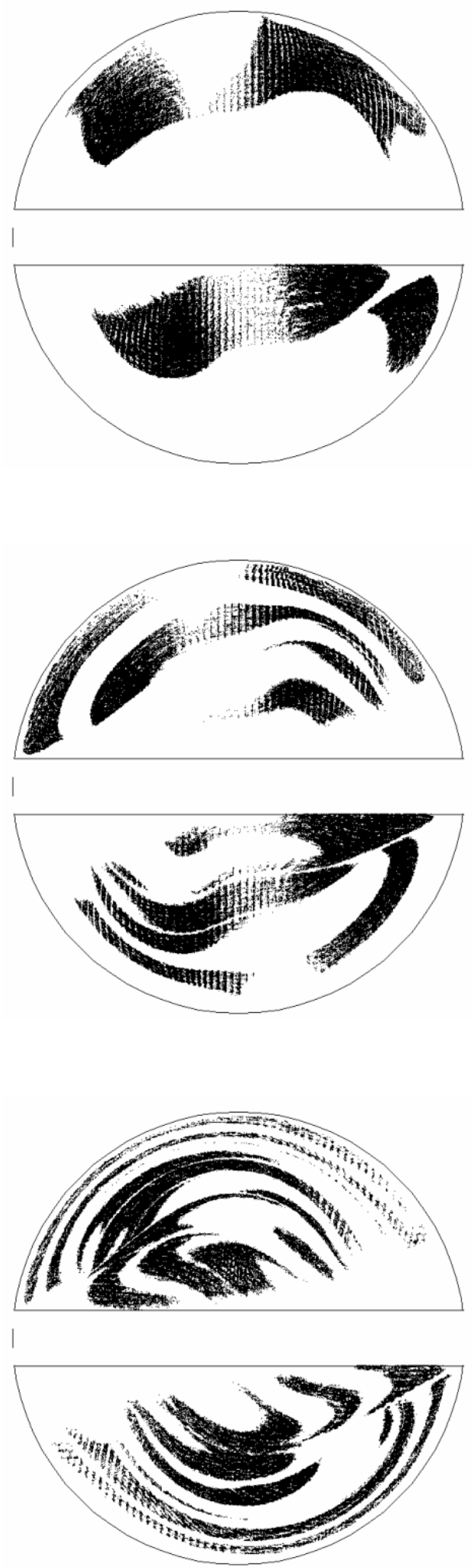

Figure 6.11: Particles locations at 2nd, 4th, and 6th mixing element for a CMC concentration level of $50 \mathrm{ppm}\left(\operatorname{Re}^{\prime}=10\right)$ 

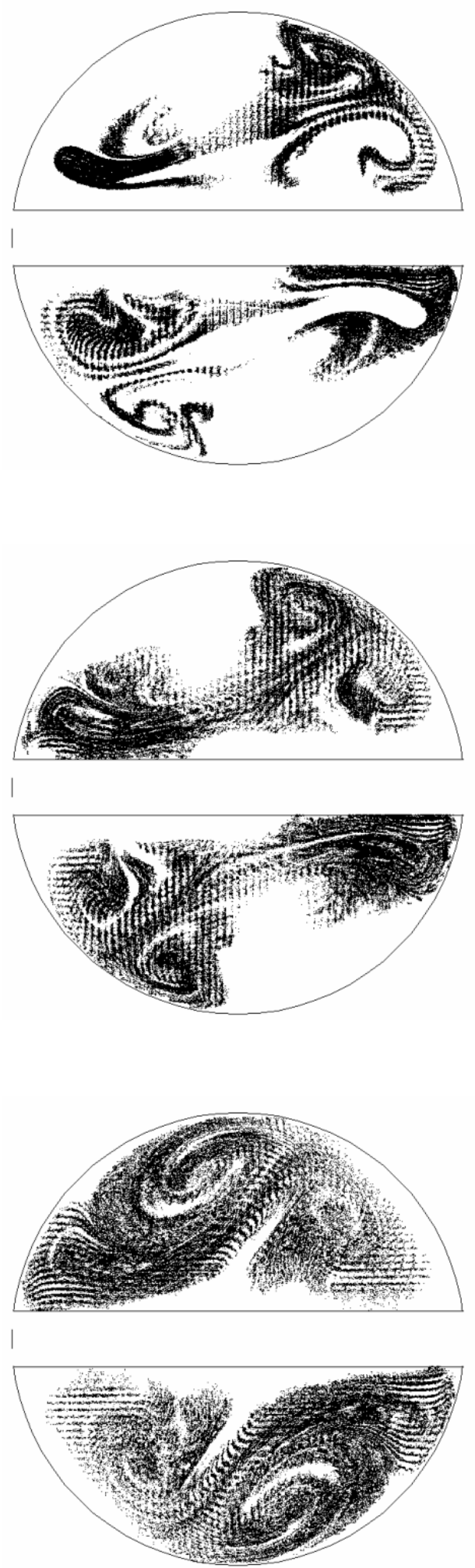

Figure 6.12: Particles locations at 2nd, 4th, and 6th mixing element for a CMC concentration level of $50 \mathrm{ppm}\left(\mathrm{Re}^{\prime}=\mathbf{1 0 0}\right)$ 

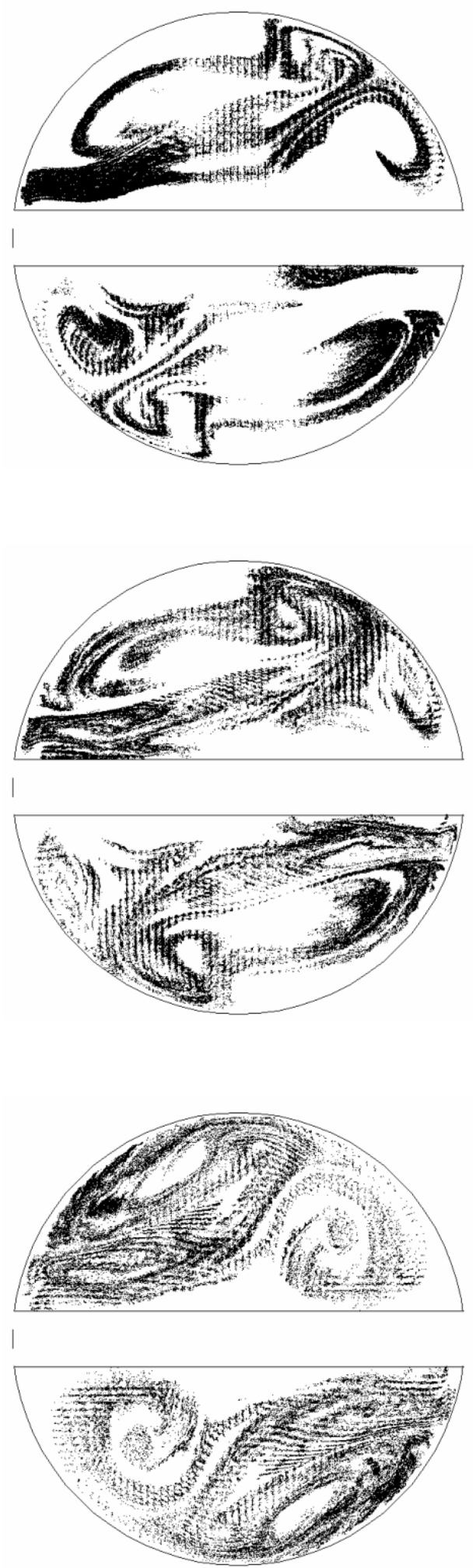

Figure 6.13: Particles locations at 2nd, 4th, and 6th mixing element for a CMC concentration level of $50 \mathrm{ppm}\left(\mathrm{Re}^{\prime}=\mathbf{1 0 0 0}\right)$ 

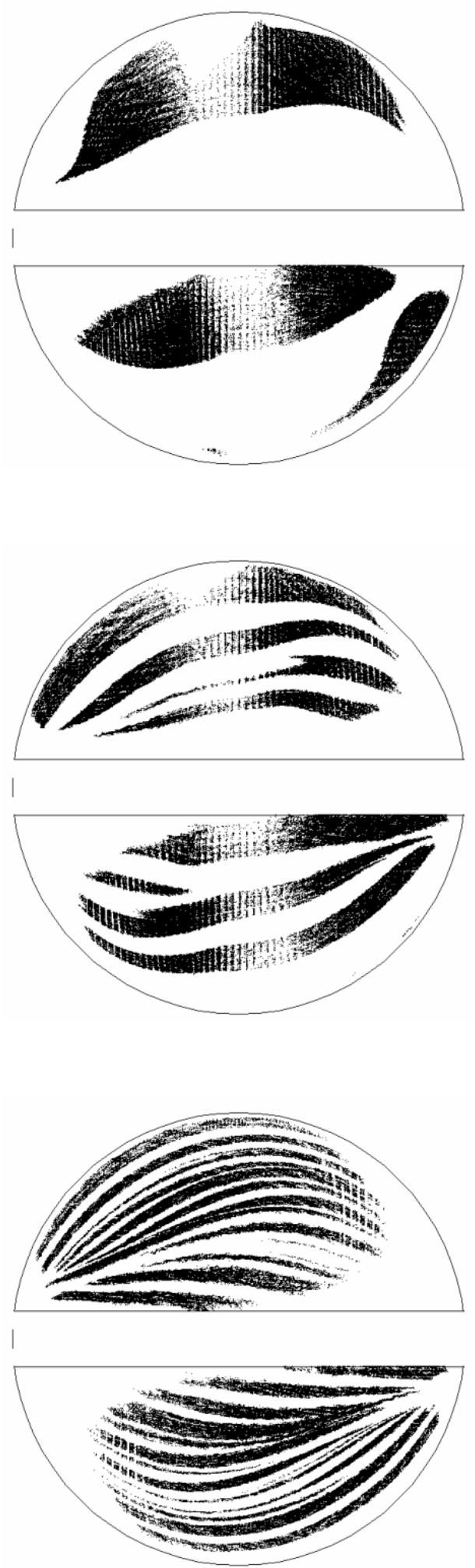

Figure 6.14: Particles locations at 2nd, 4th, and 6th mixing element for a CMC concentration level of $500 \mathrm{ppm}\left(\mathrm{Re}^{\prime}=\mathbf{0 . 1}\right)$ 

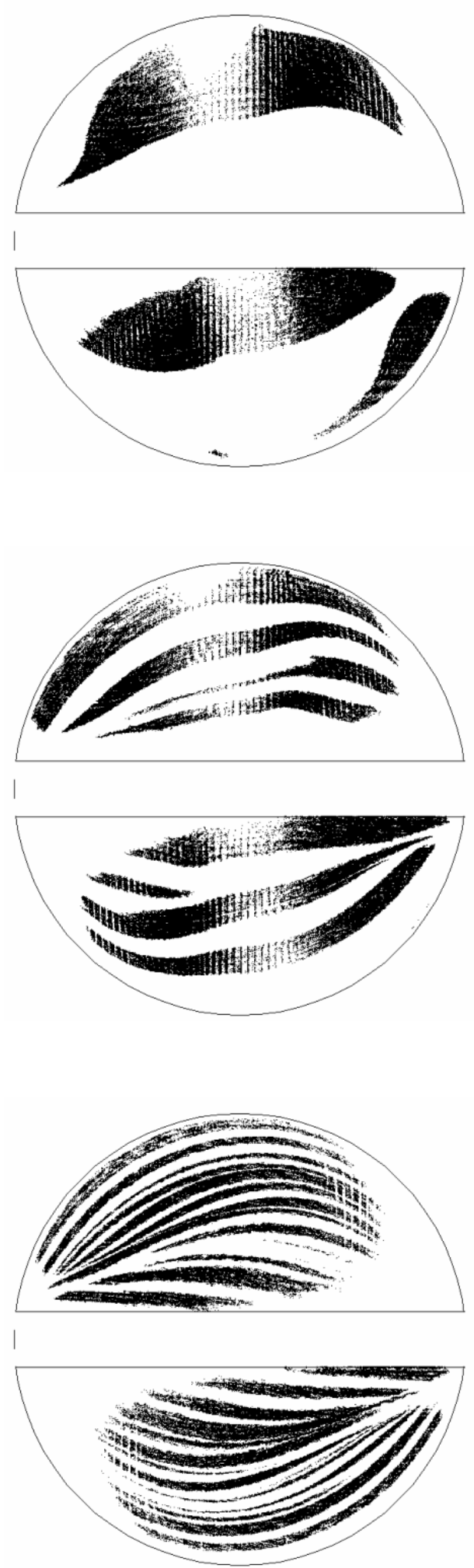

Figure 6.15: Particles locations at 2nd, 4th, and 6th mixing element for a CMC concentration level of $500 \mathrm{ppm}\left(\operatorname{Re}^{\prime}=1\right)$ 

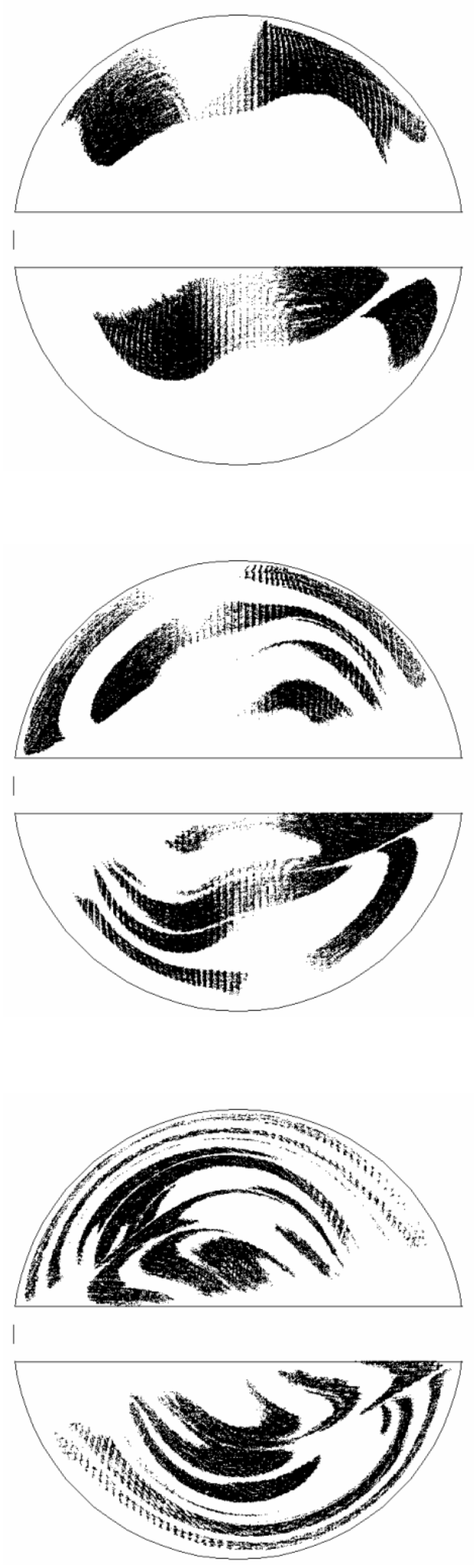

Figure 6.16: Particles locations at 2nd, 4th, and 6th mixing element for a CMC concentration level of $500 \mathrm{ppm}\left(\operatorname{Re}^{\prime}=10\right)$ 

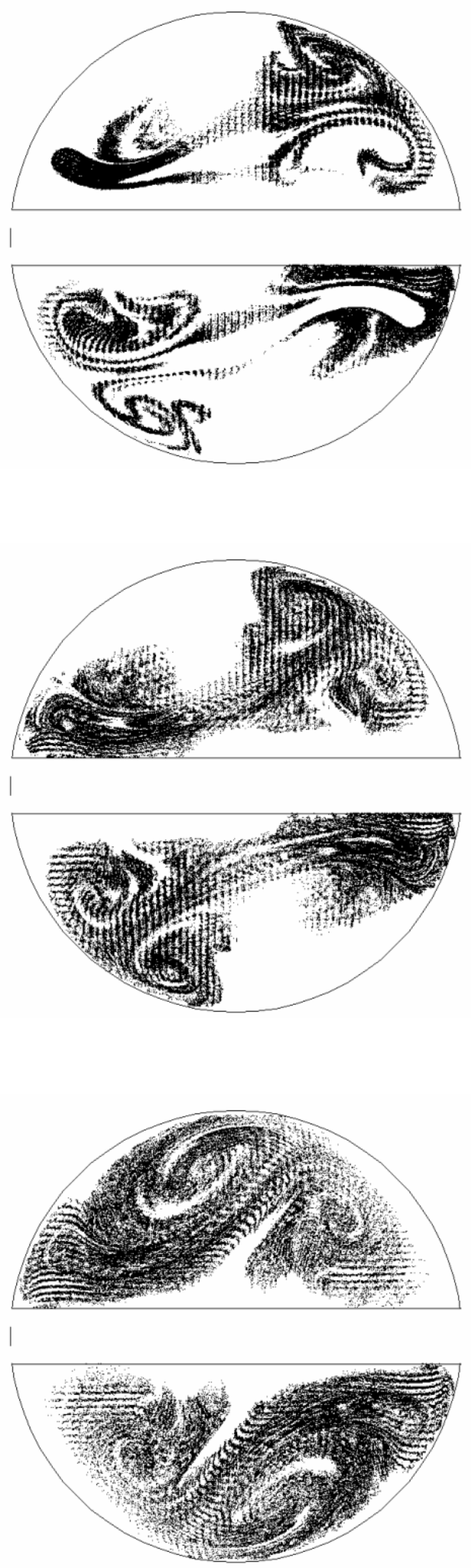

Figure 6.17: Particles locations at 2nd, 4th, and 6th mixing element for a CMC concentration level of $500 \mathrm{ppm}\left(\mathrm{Re}^{\prime}=\mathbf{1 0 0}\right)$ 

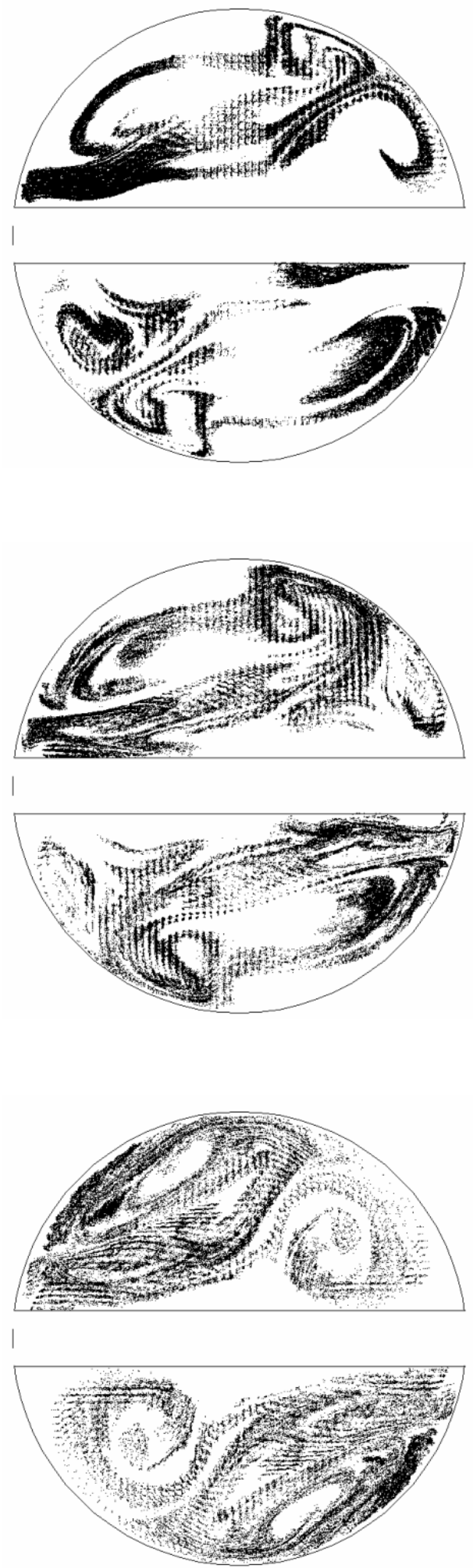

Figure 6.18: Particles locations at 2nd, 4th, and 6th mixing element for a CMC concentration level of $500 \mathrm{ppm}\left(\mathrm{Re}^{\prime}=\mathbf{1 0 0 0}\right)$ 

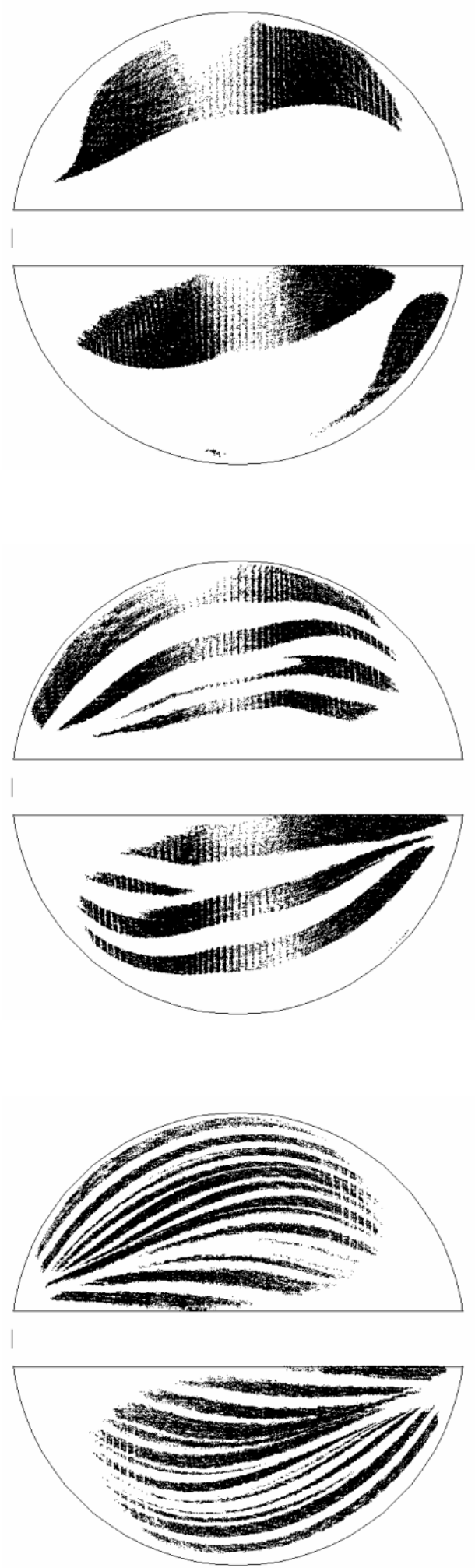

Figure 6.19: Particles locations at 2nd, 4th, and 6th mixing element for a CMC concentration level of $5000 \mathrm{ppm}\left(\operatorname{Re}^{\prime}=\mathbf{0 . 1}\right)$ 

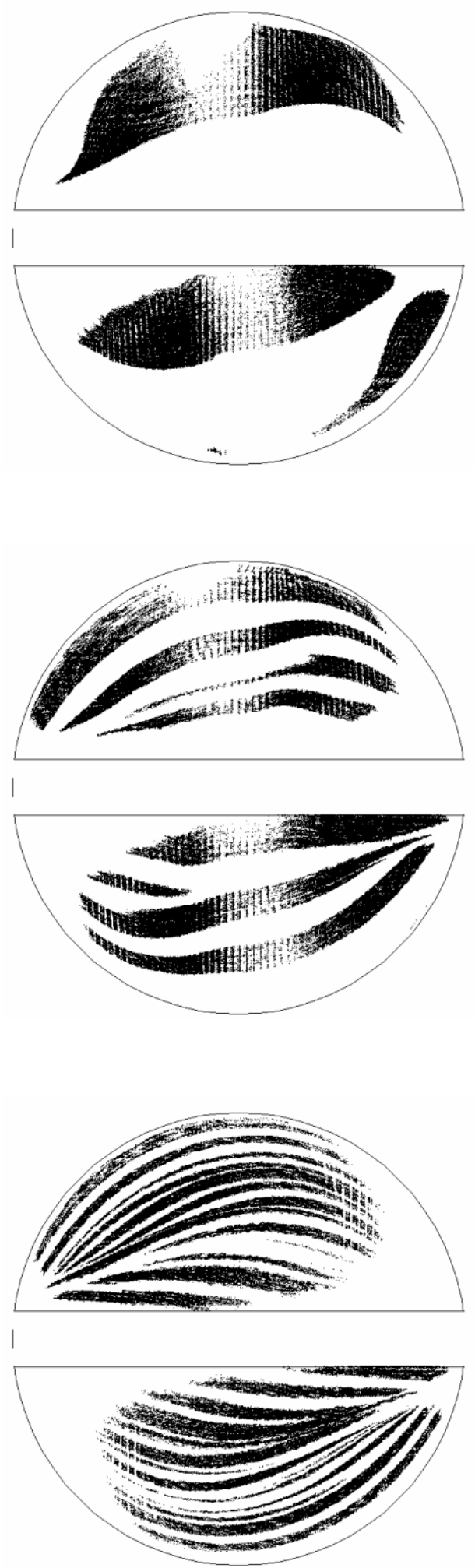

Figure 6.20: Particles locations at 2nd, 4th, and 6th mixing element for a CMC concentration level of $5000 \mathrm{ppm}\left(\operatorname{Re}^{\prime}=1\right)$ 

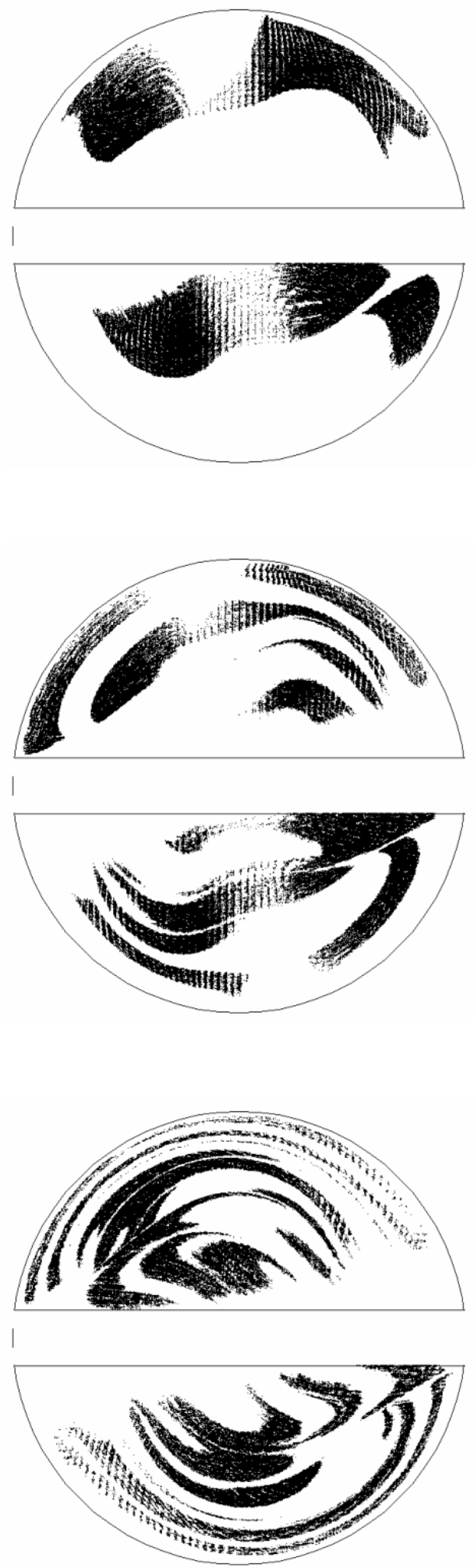

Figure 6.21: Particles locations at 2nd, 4th, and 6th mixing element for a CMC concentration level of $5000 \mathrm{ppm}\left(\operatorname{Re}^{\prime}=10\right)$ 

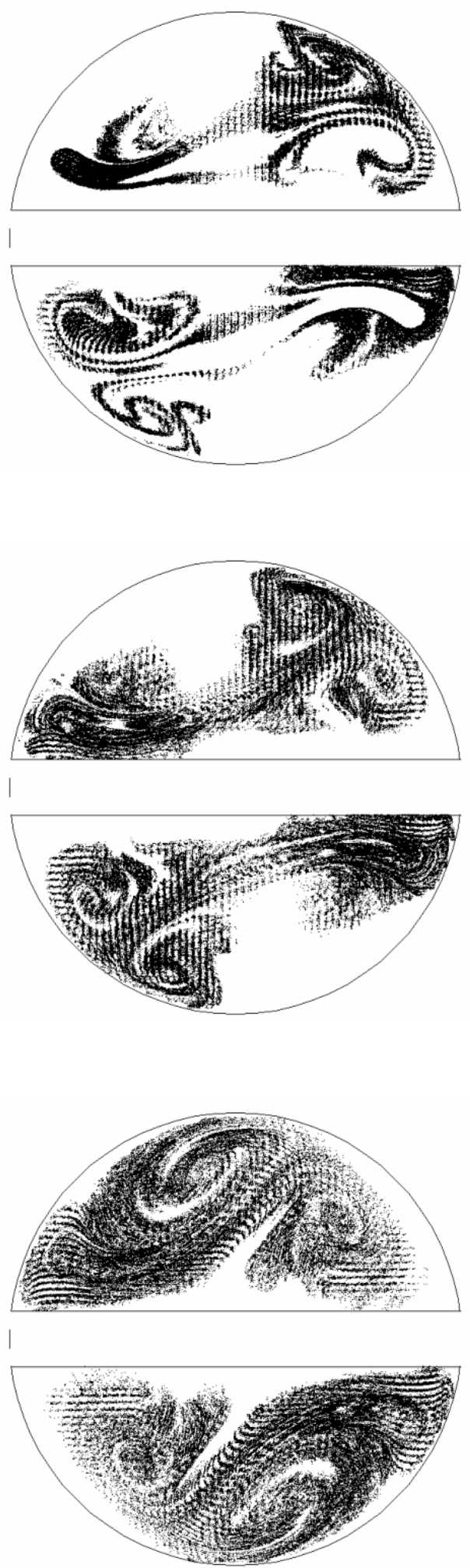

Figure 6.22: Particles locations at 2nd, 4th, and 6th mixing element for a CMC concentration level of $5000 \mathrm{ppm}\left(\operatorname{Re}^{\prime}=100\right)$ 

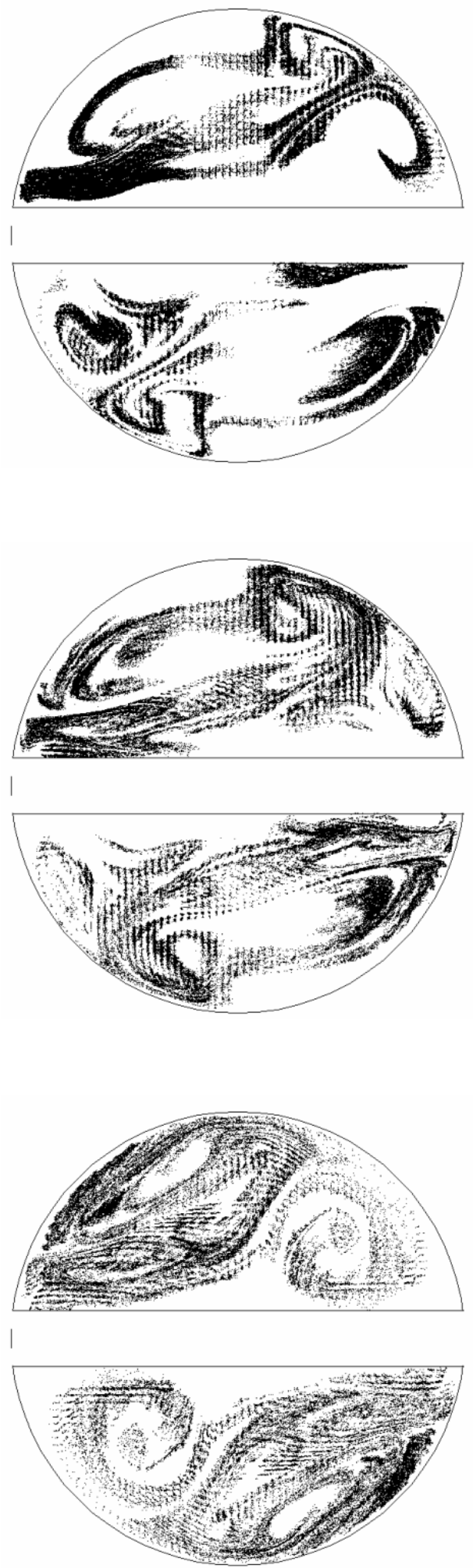

Figure 6.23: Particles locations at 2nd, 4th, and 6th mixing element for a CMC concentration level of $5000 \mathrm{ppm}\left(\mathrm{Re}^{\prime}=\mathbf{1 0 0 0}\right)$ 


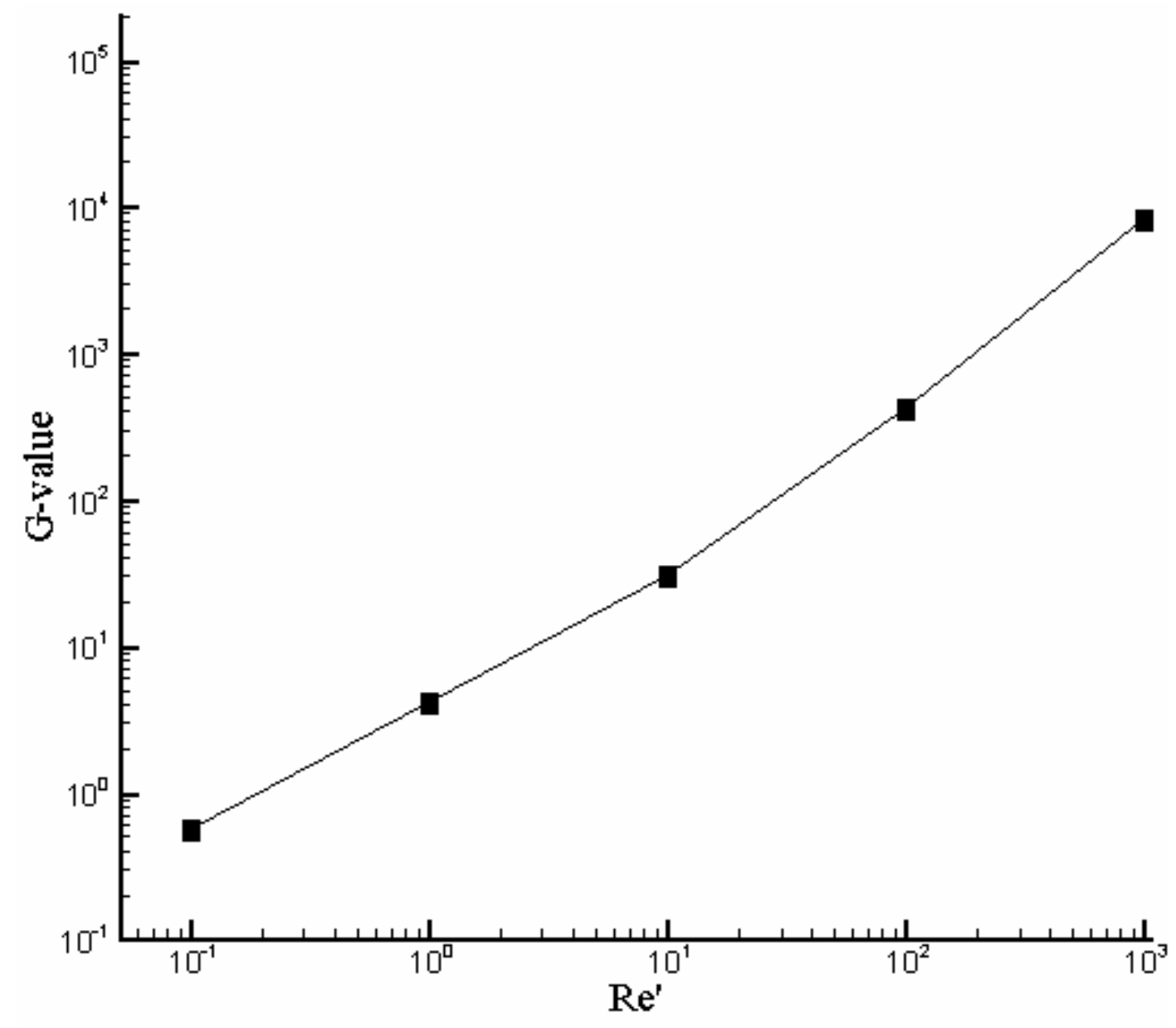

Figure 6.24: G-values for a six-element mixer $(1 / s)$ CMC concentration level: 50 ppm 


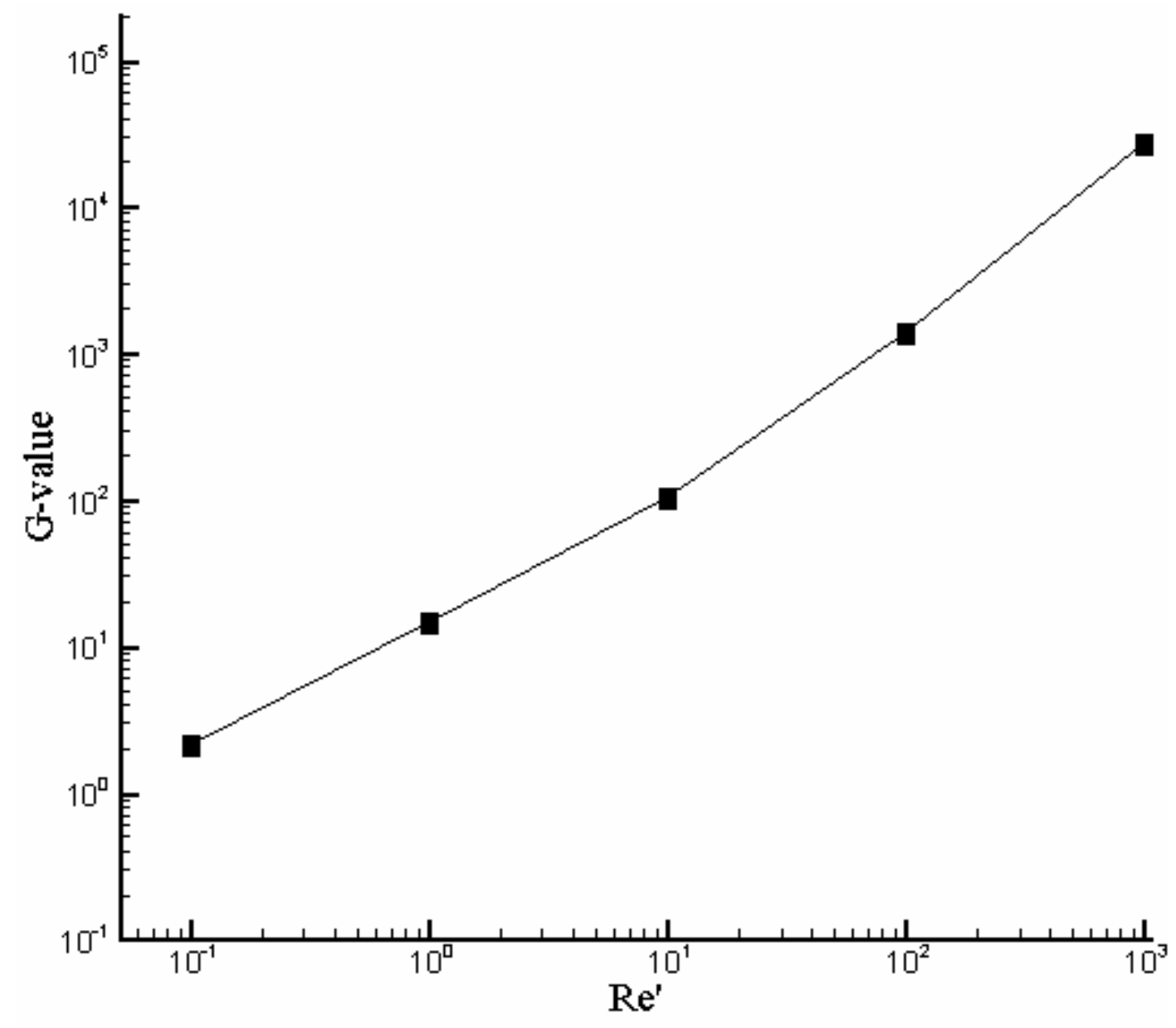

Figure 6.25: G-values for a six-element mixer $(1 / s)$

CMC concentration level: 500 ppm 


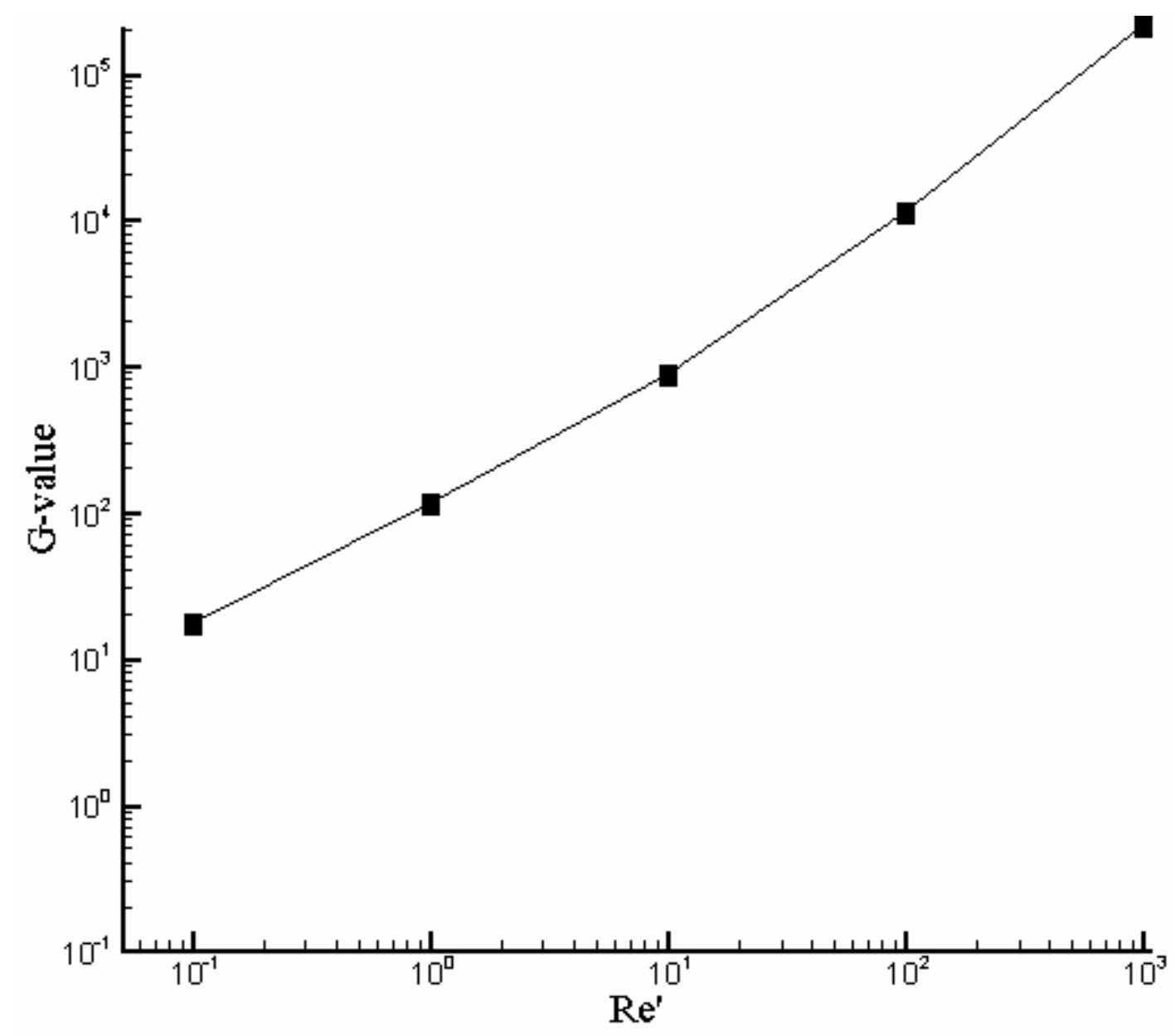

Figure 6.26: G-values for a six-element mixer $(1 / s$ )

CMC concentration level: 5000 ppm 


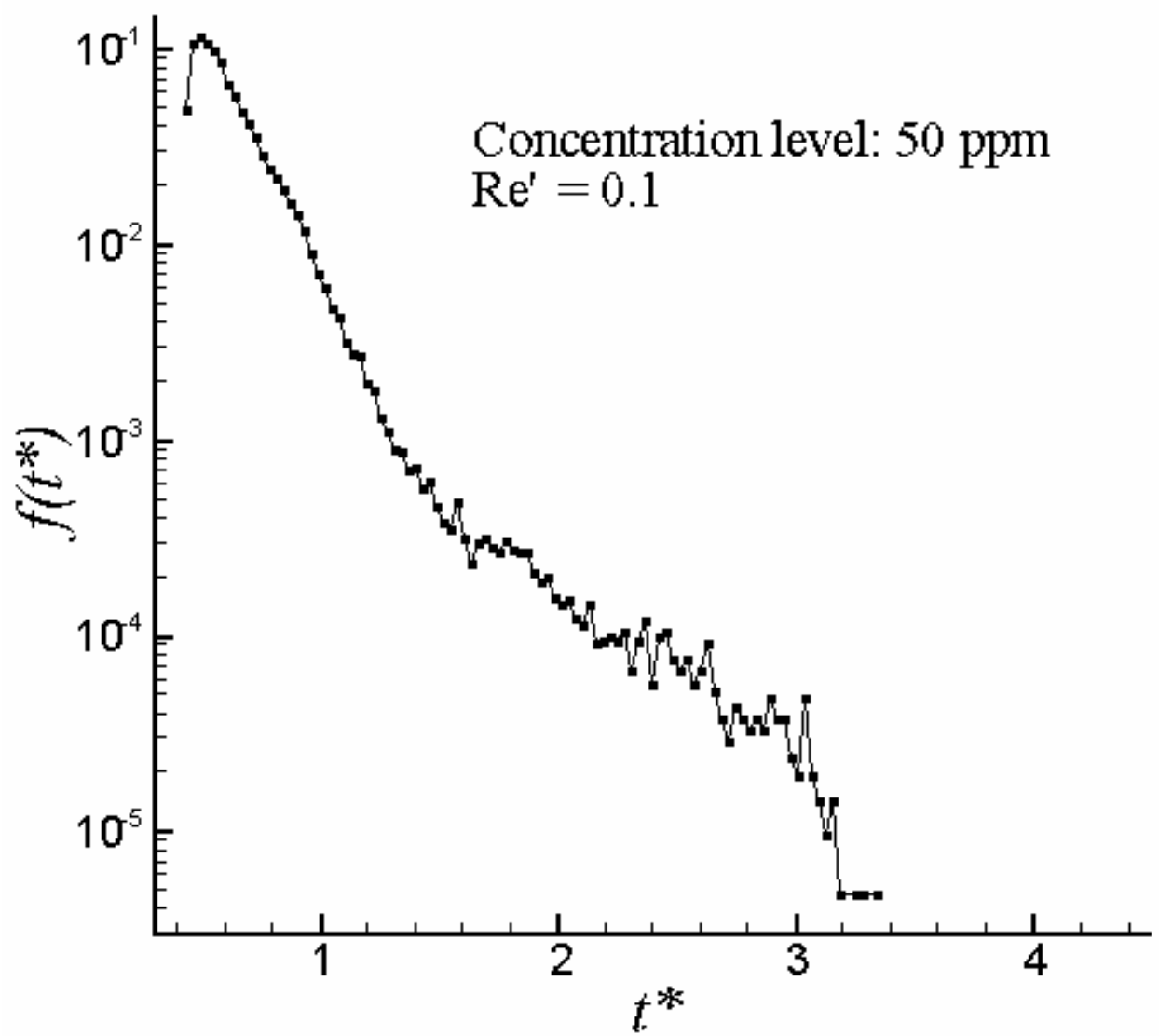

Figure 6.27: Distribution function for CMC solutions flow in the six-element static mixer $\left(\operatorname{Re}^{\prime}=0.1\right.$, Concentration level: $\left.50 \mathrm{ppm}, d t^{*}=\mathbf{0 . 0 1}\right)$ 


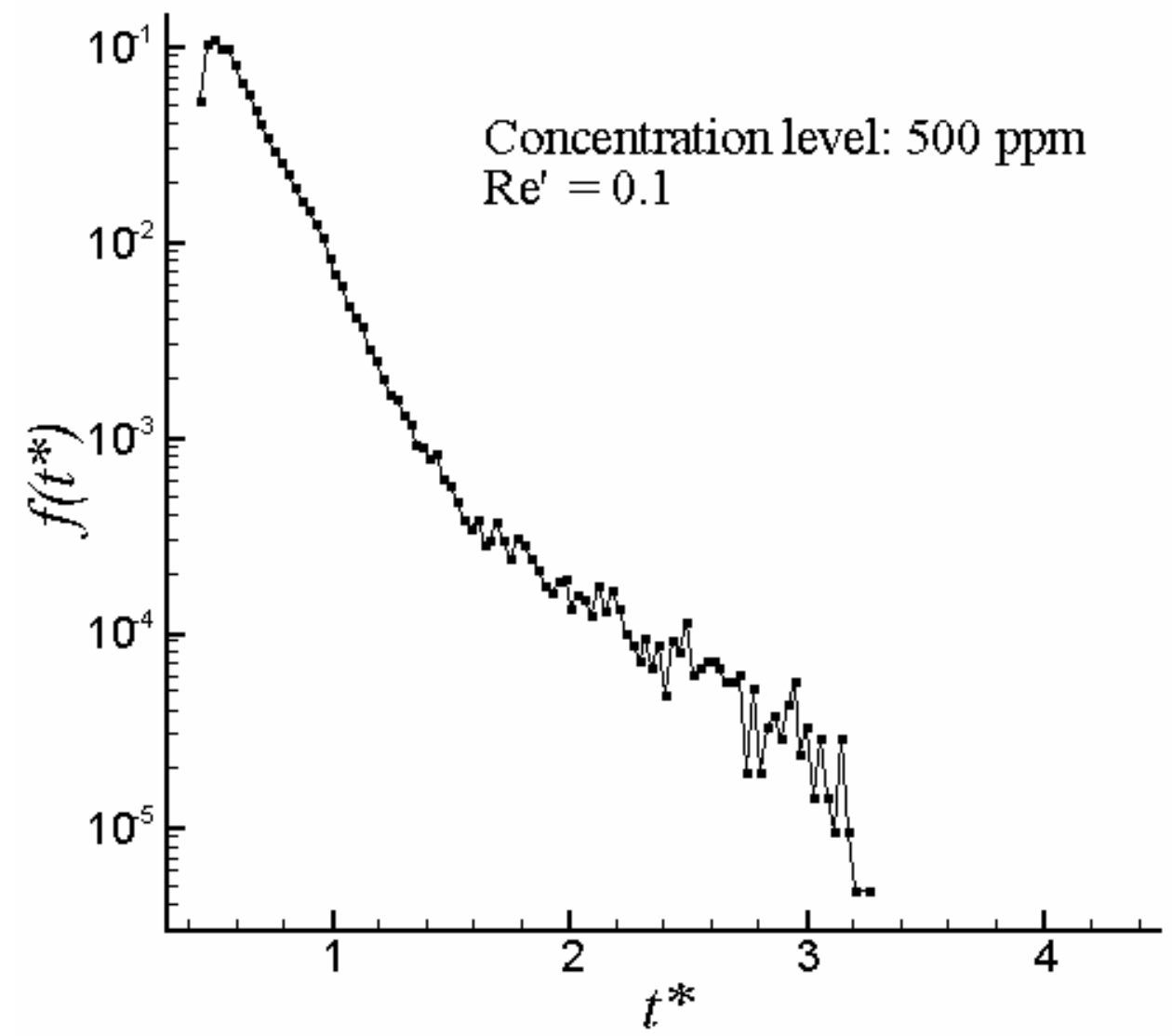

Figure 6.28: Distribution function for CMC solutions flow in the six-element static mixer $\left(\operatorname{Re}^{\prime}=0.1\right.$, Concentration level: $\left.500 \mathrm{ppm}, d t^{*}=\mathbf{0 . 0 1}\right)$ 


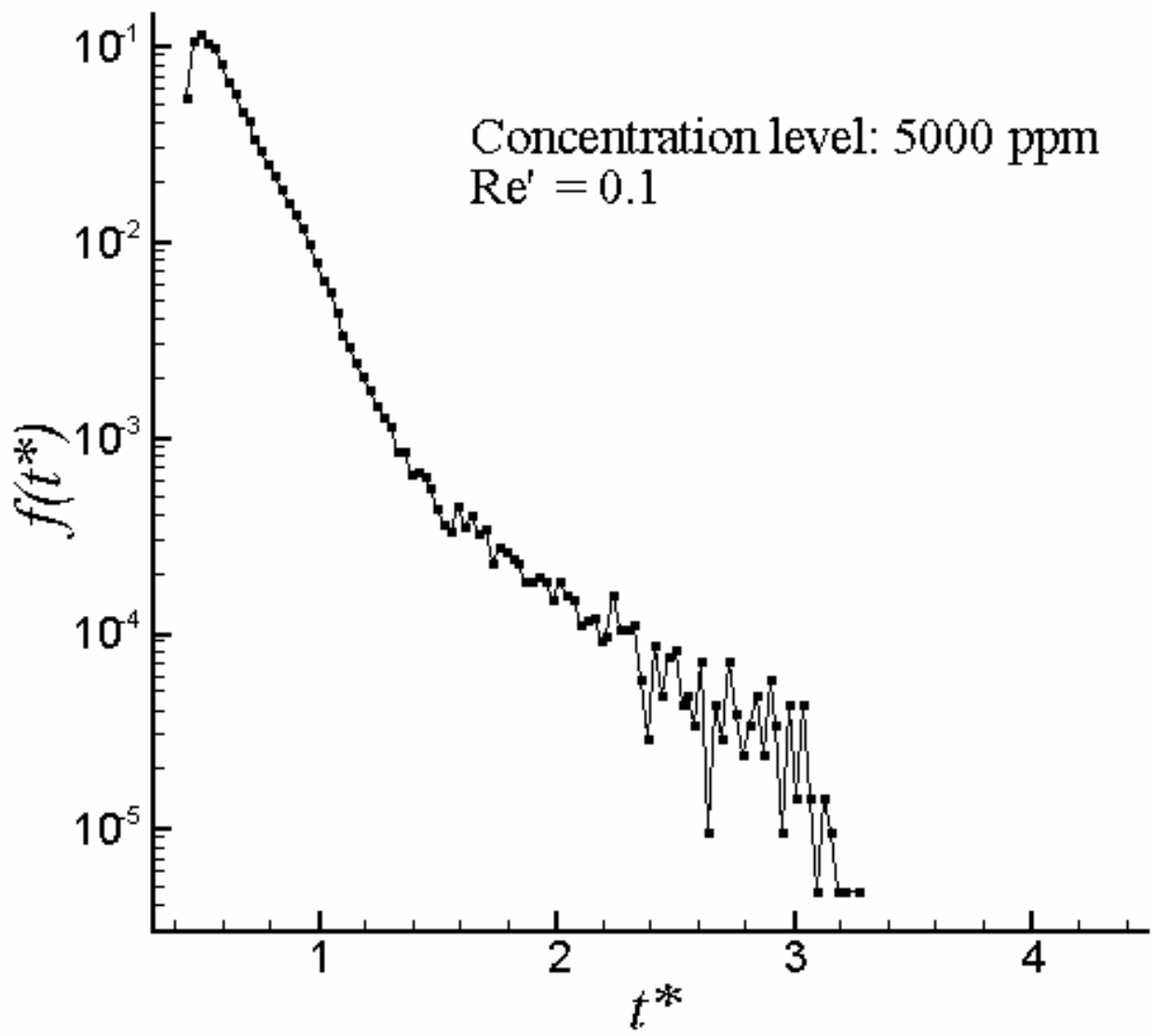

Figure 6.29: Distribution function for CMC solutions flow in the six-element static mixer $\left(\operatorname{Re}^{\prime}=0.1\right.$, Concentration level: $\left.5000 \mathrm{ppm}, d t^{*}=\mathbf{0 . 0 1}\right)$ 


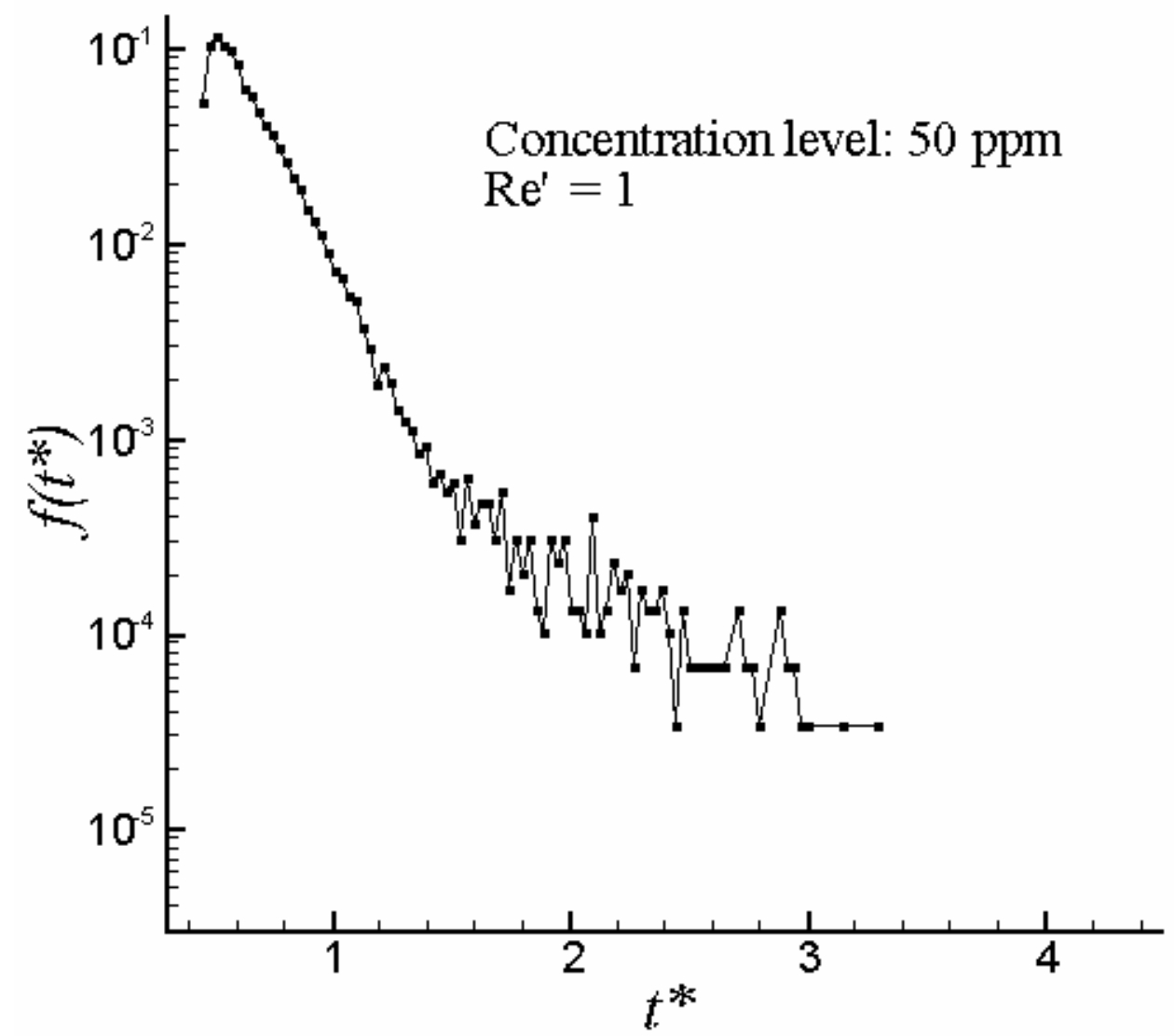

Figure 6.30: Distribution function for CMC solutions flow in the six-element static mixer $\left(\operatorname{Re}^{\prime}=1\right.$, Concentration level: $50 \mathrm{ppm}, d t^{*}=0.01$ ) 


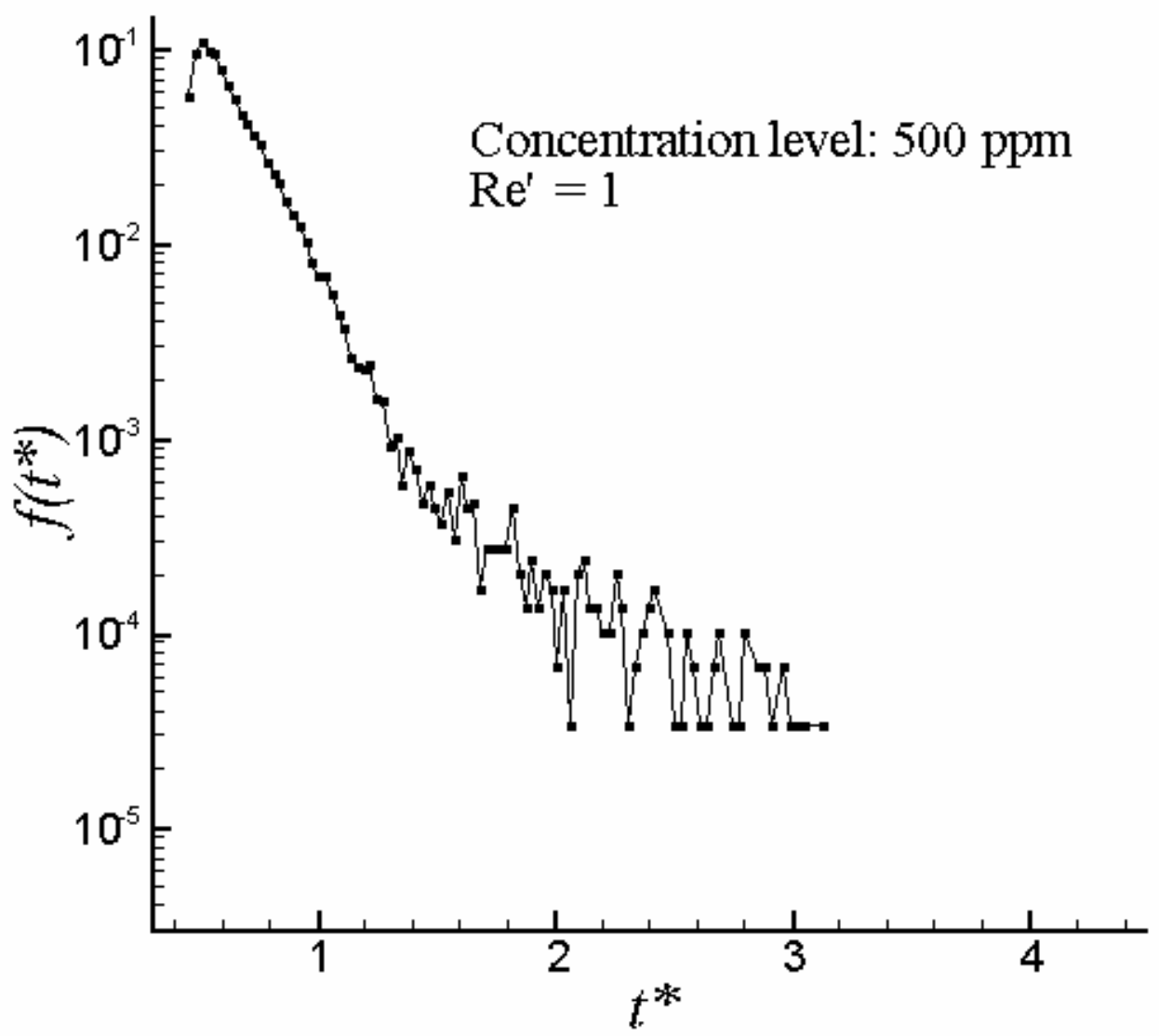

Figure 6.31: Distribution function for CMC solutions flow in the six-element static mixer $\left(\operatorname{Re}^{\prime}=1\right.$, Concentration level: $\left.500 \mathrm{ppm}, d t^{*}=0.01\right)$ 


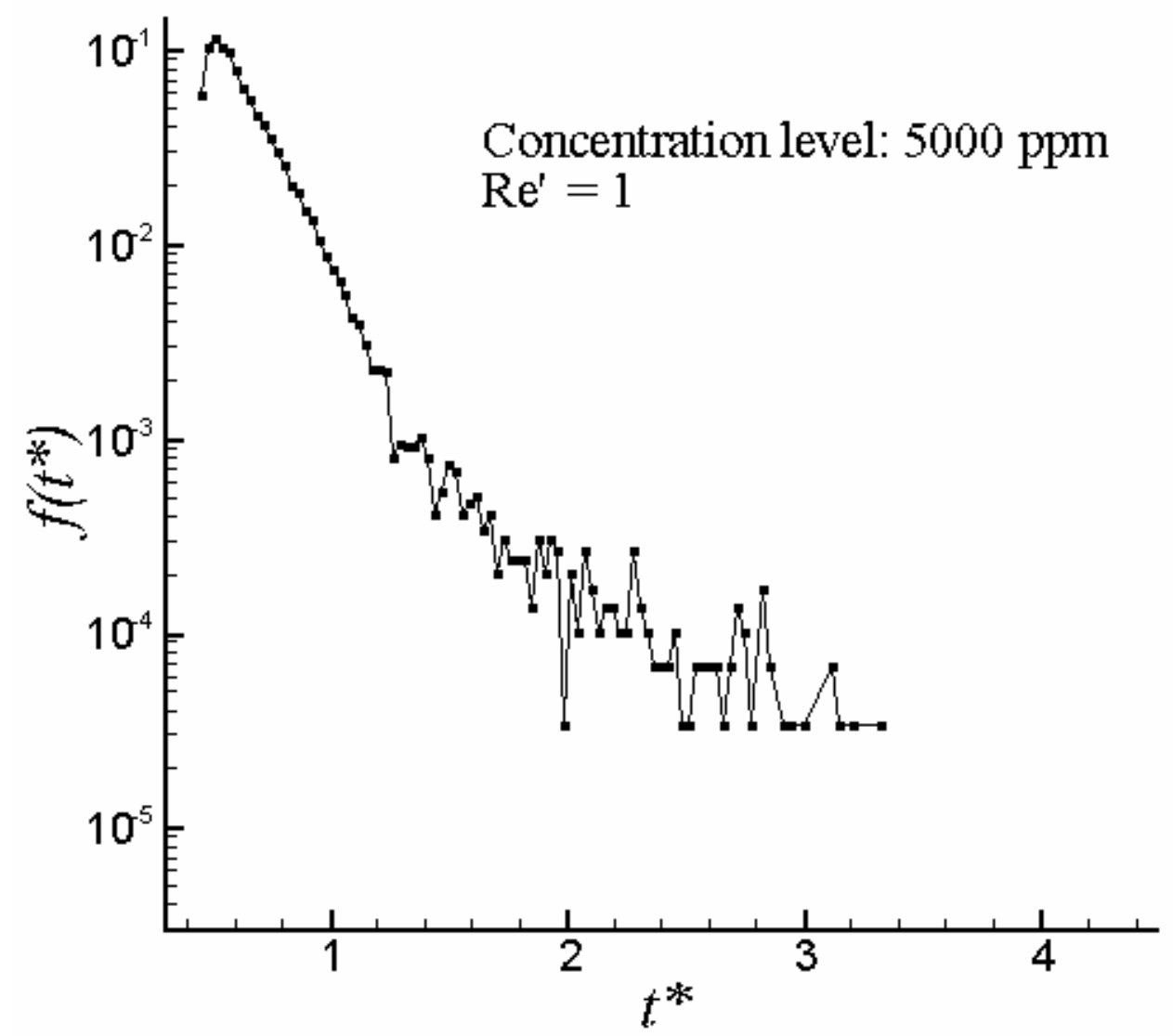

Figure 6.32: Distribution function for CMC solutions flow in the six-element static mixer $\left(\operatorname{Re}^{\prime}=1\right.$, Concentration level: $\left.5000 \mathrm{ppm}, d t^{*}=0.01\right)$ 


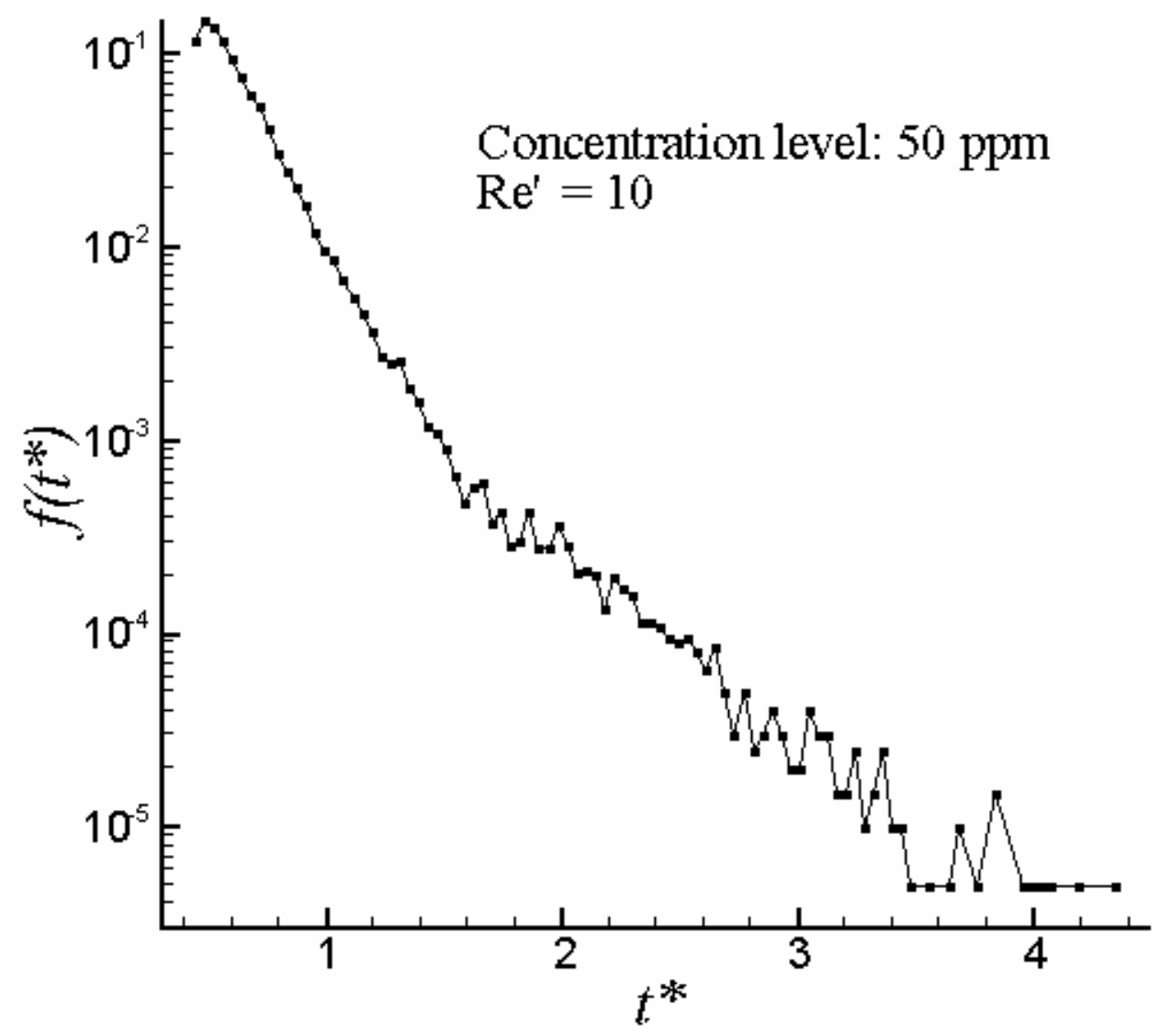

Figure 6.33: Distribution function for CMC solutions flow in the six-element static mixer $\left(\operatorname{Re}^{\prime}=10\right.$, Concentration level: $\left.50 \mathrm{ppm}, d t^{*}=0.01\right)$ 


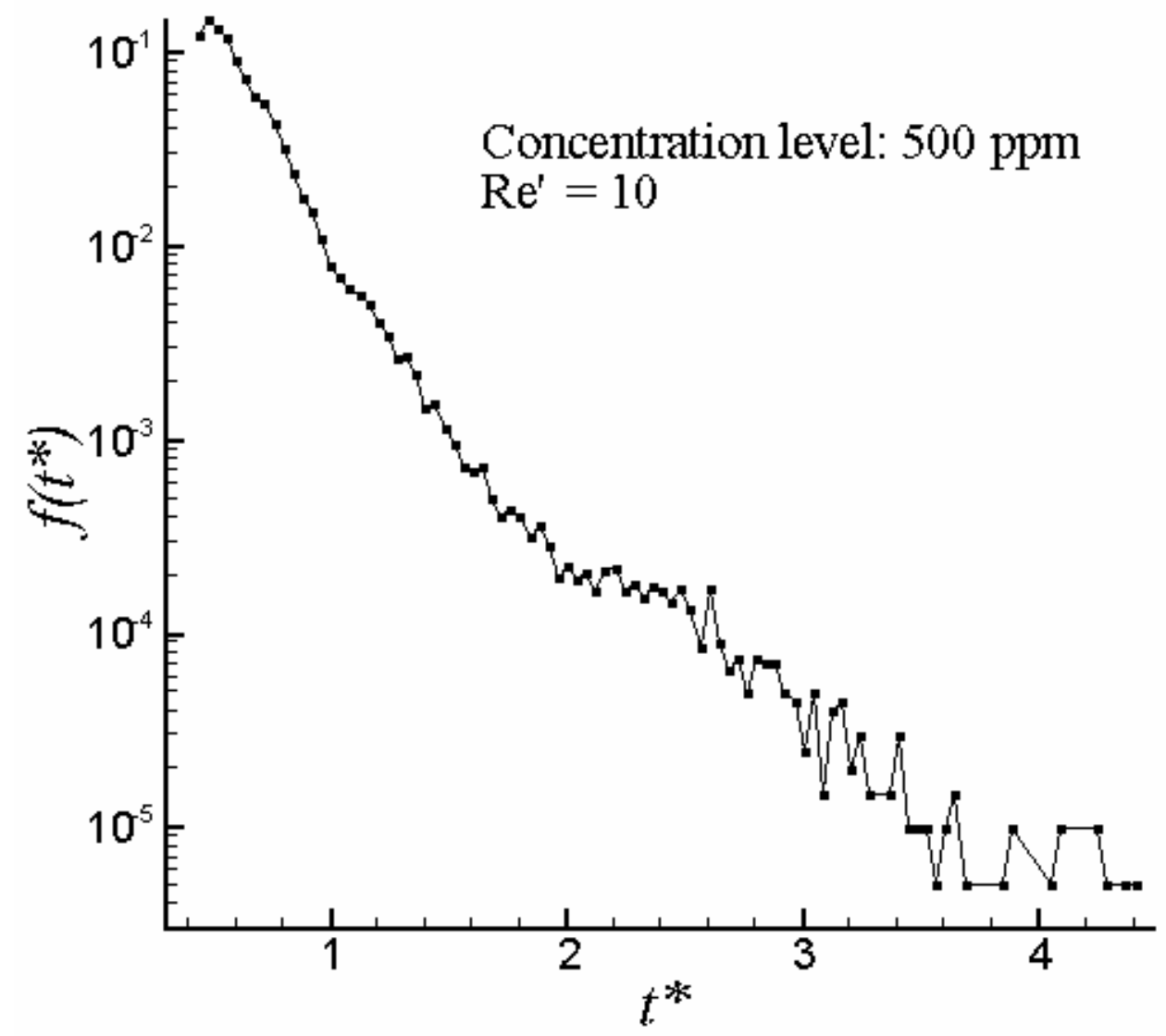

Figure 6.34: Distribution function for CMC solutions flow in the six-element static mixer $\left(\operatorname{Re}^{\prime}=10\right.$, Concentration level: $\left.500 \mathrm{ppm}, d t^{*}=\mathbf{0 . 0 1}\right)$ 


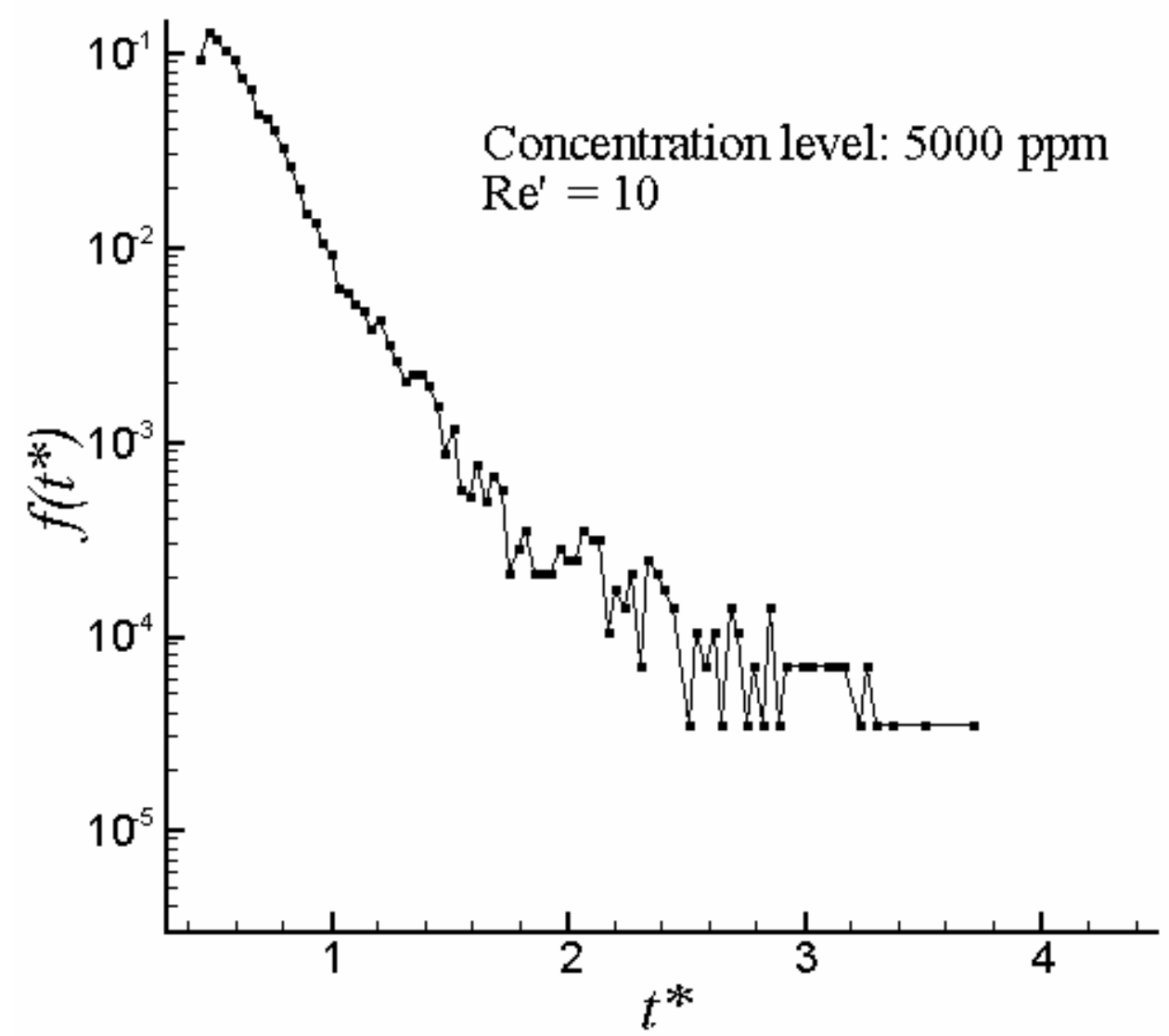

Figure 6.35: Distribution function for CMC solutions flow in the six-element static mixer $\left(\operatorname{Re}^{\prime}=10\right.$, Concentration level: $\left.5000 \mathrm{ppm}, d t^{*}=0.01\right)$ 


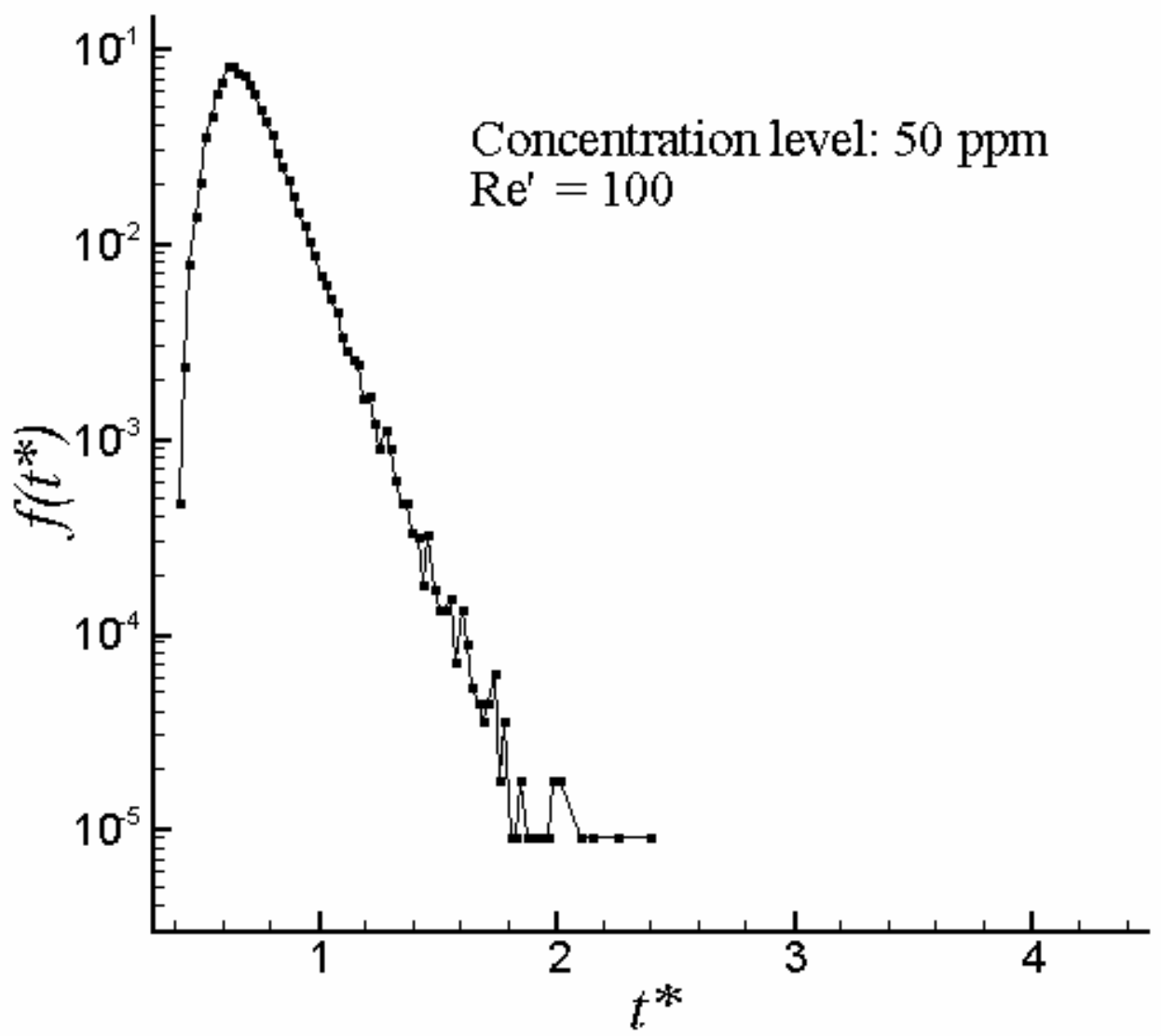

Figure 6.36: Distribution function for CMC solutions flow in the six-element static mixer $\left(\operatorname{Re}^{\prime}=100\right.$, Concentration level: $50 \mathrm{ppm}, d t^{*}=0.01$ ) 


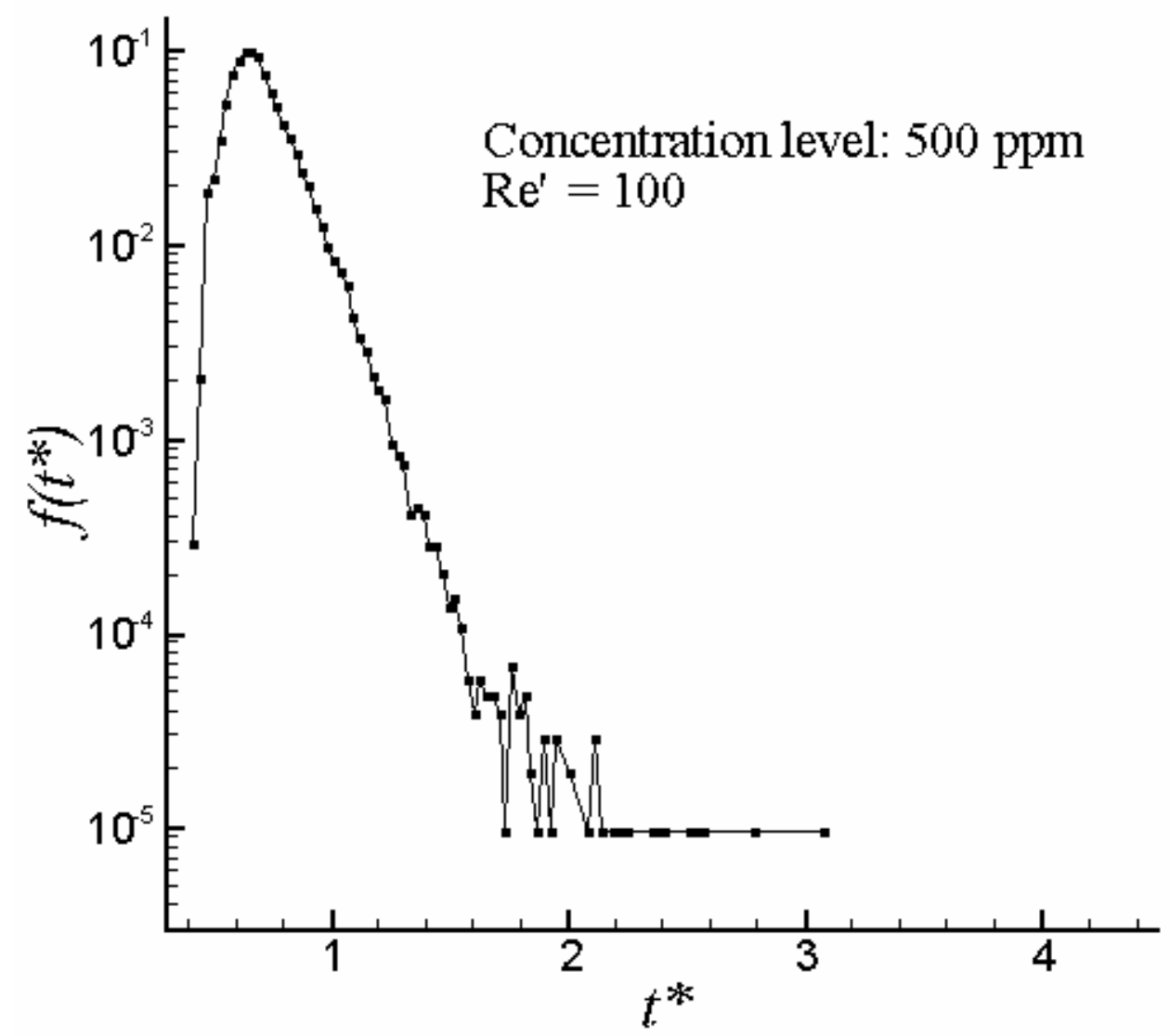

Figure 6.37: Distribution function for CMC solutions flow in the six-element static mixer $\left(\operatorname{Re}^{\prime}=100\right.$, Concentration level: $\left.500 \mathrm{ppm}, d t^{*}=0.01\right)$ 


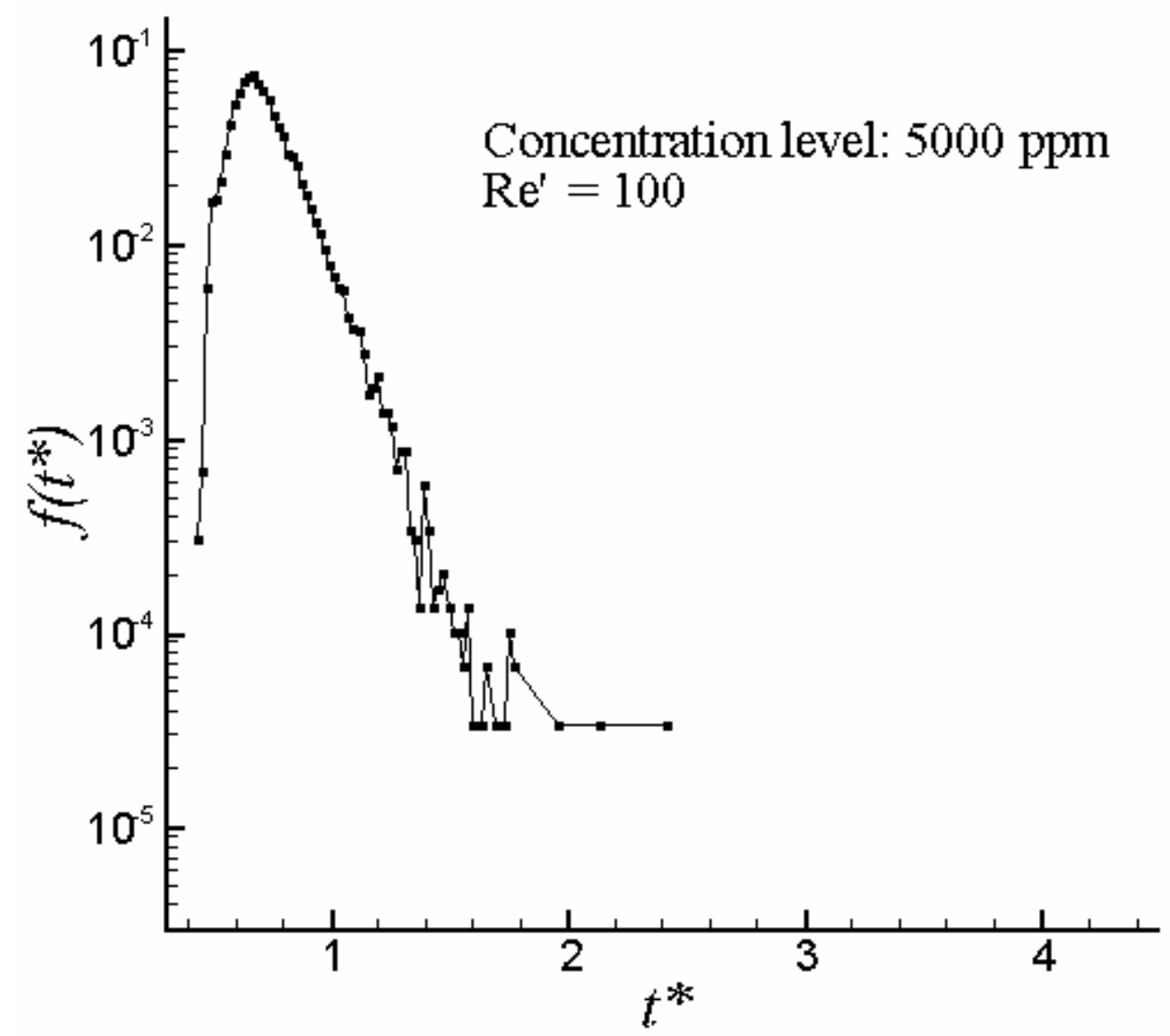

Figure 6.38: Distribution function for CMC solutions flow in the six-element static mixer $\left(\operatorname{Re}^{\prime}=100\right.$, Concentration level: $\left.5000 \mathrm{ppm}, d t^{*}=\mathbf{0 . 0 1}\right)$ 


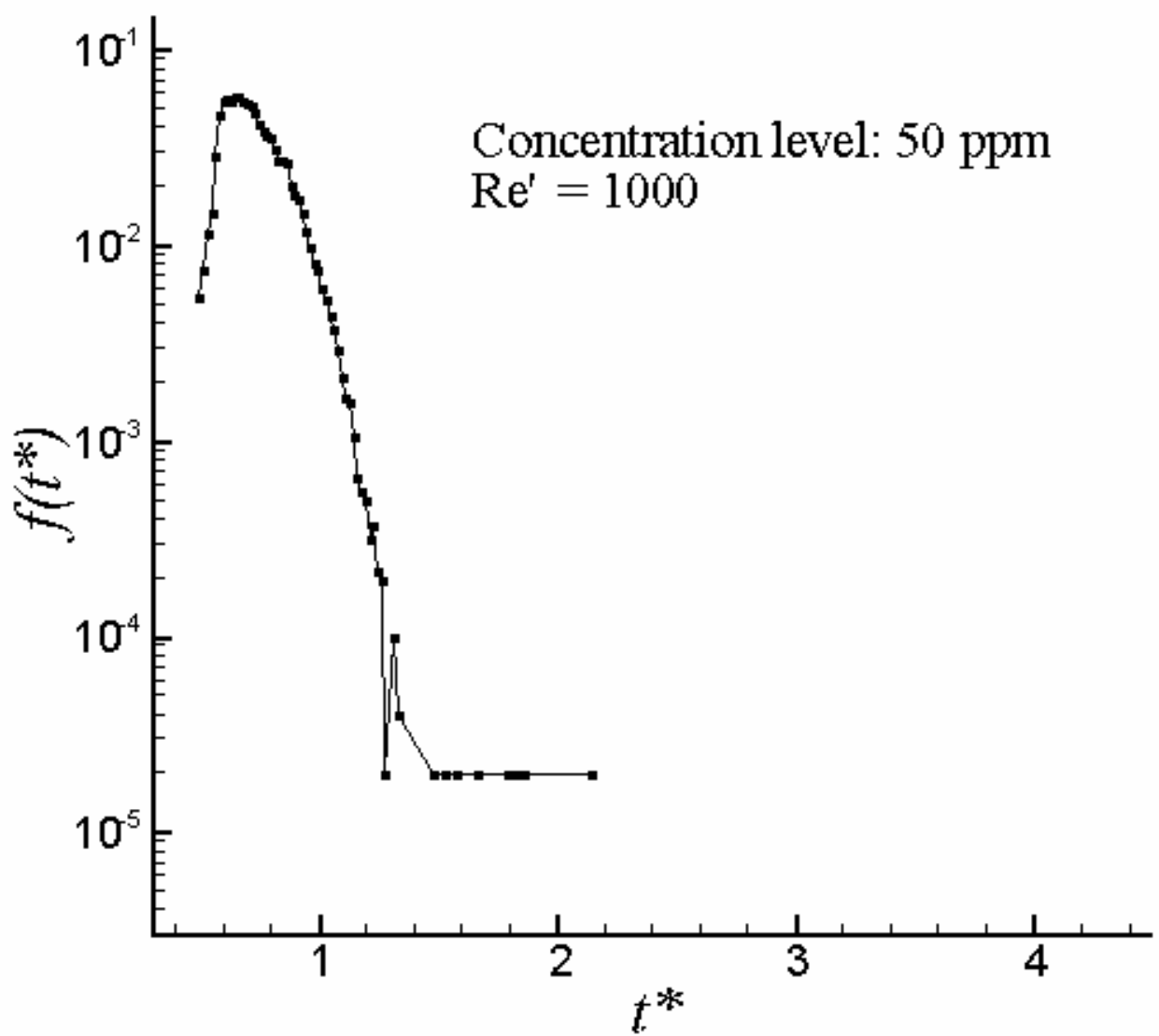

Figure 6.39: Distribution function for $\mathrm{CMC}$ solutions flow in the six-element static mixer $\left(\operatorname{Re}^{\prime}=1000\right.$, Concentration level: $\left.50 \mathrm{ppm}, d t^{*}=0.01\right)$ 


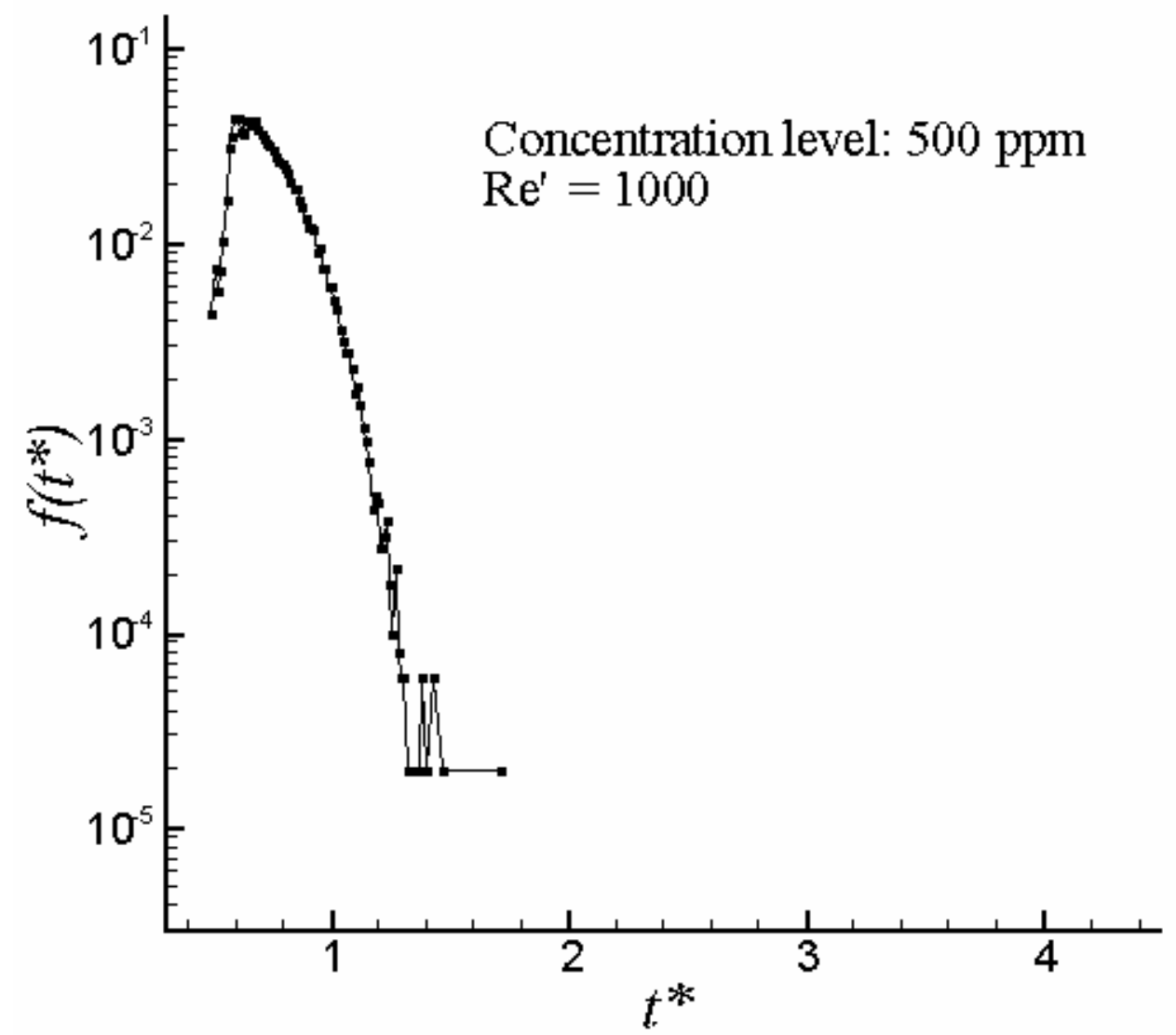

Figure 6.40: Distribution function for CMC solutions flow in the six-element static mixer $\left(\operatorname{Re}^{\prime}=1000\right.$, Concentration level: $\left.500 \mathrm{ppm}, d t^{*}=0.01\right)$ 


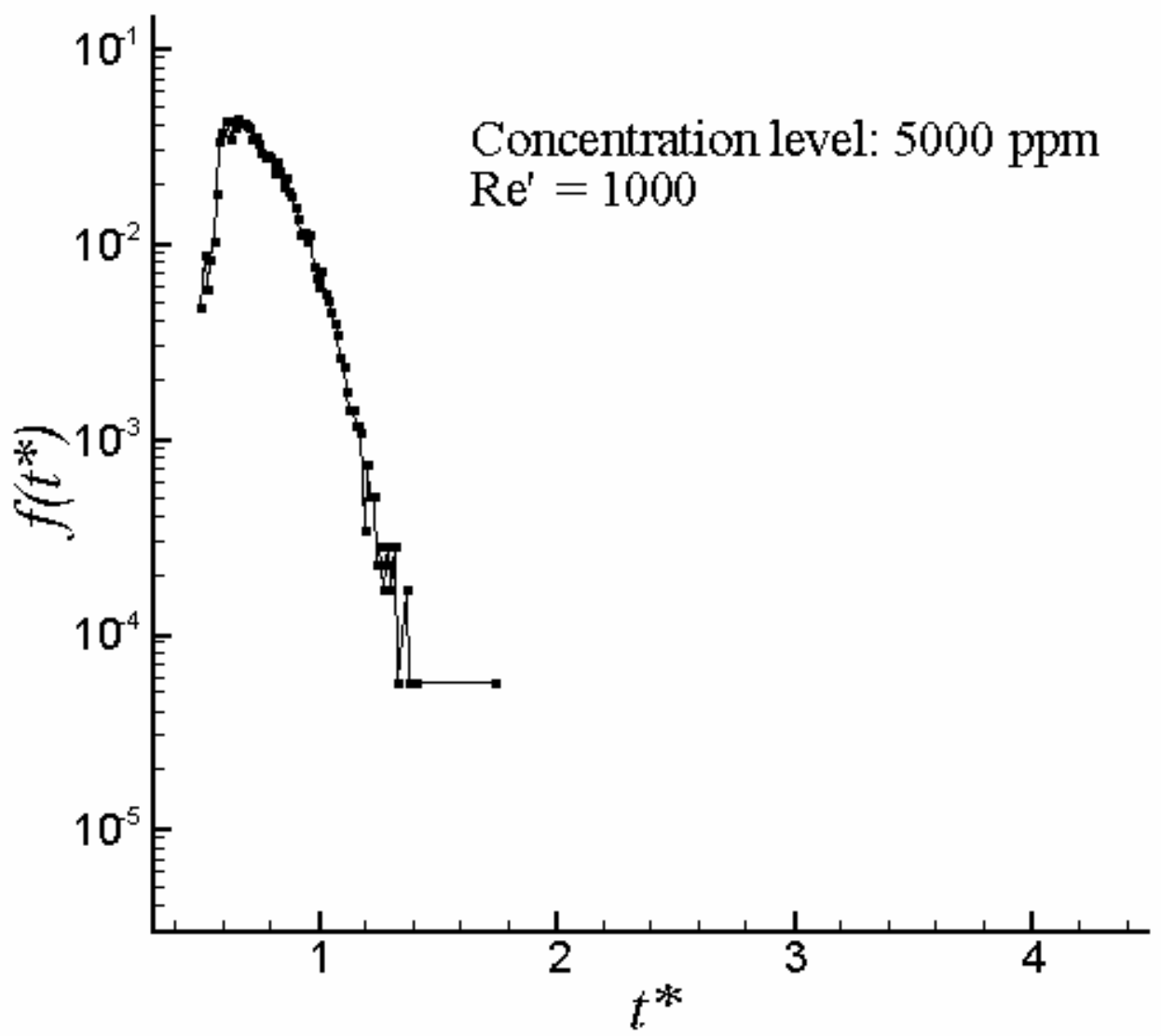

Figure 6.41: Distribution function for CMC solutions flow in the six-element static mixer $\left(\operatorname{Re}^{\prime}=1000\right.$, Concentration level: $\left.5000 \mathrm{ppm}, d t^{*}=0.01\right)$ 


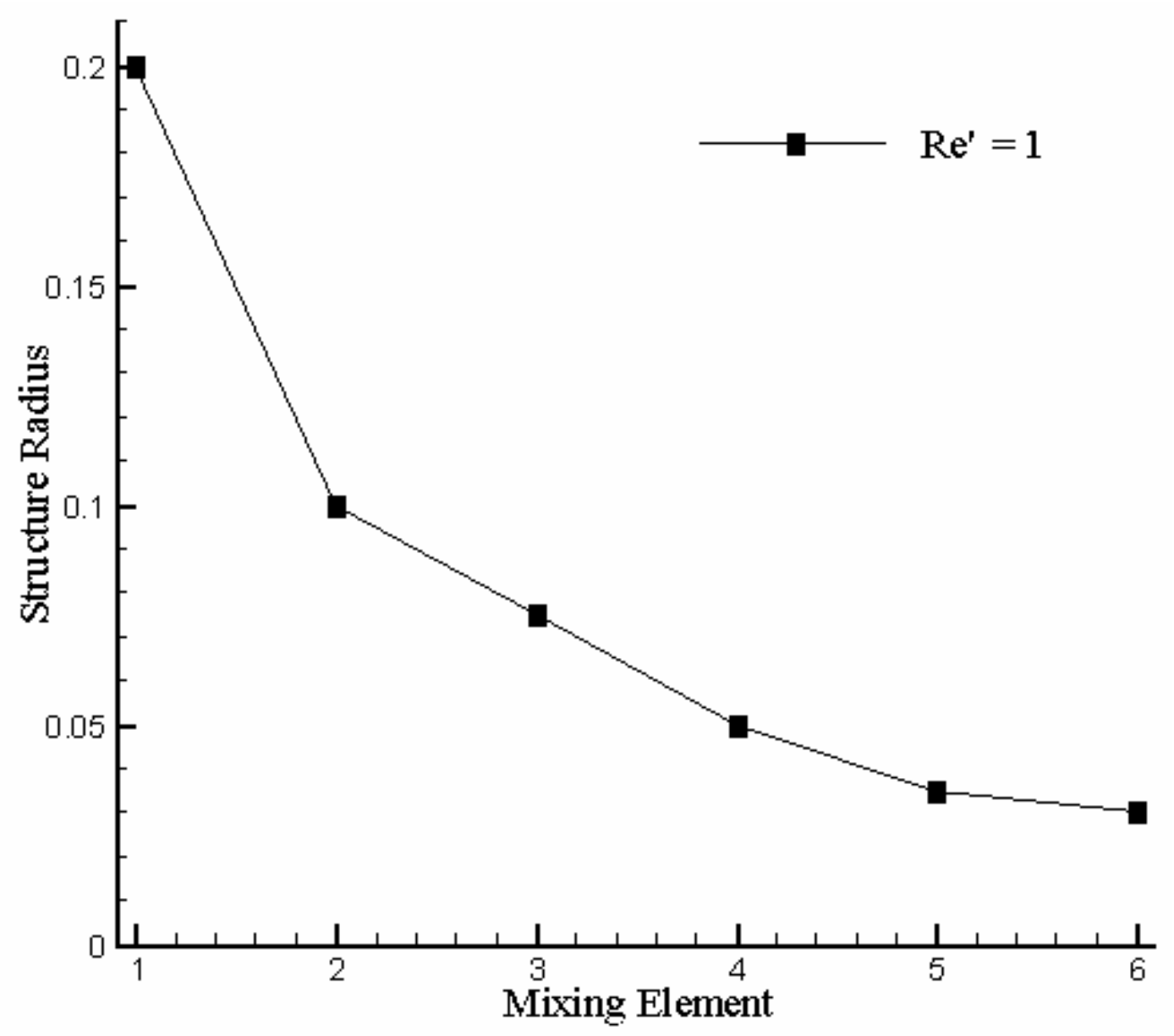

Figure 6.42: Structure radii for $\mathrm{Re}^{\prime}=1$ (CMC concentration level: 5000 ppm) 


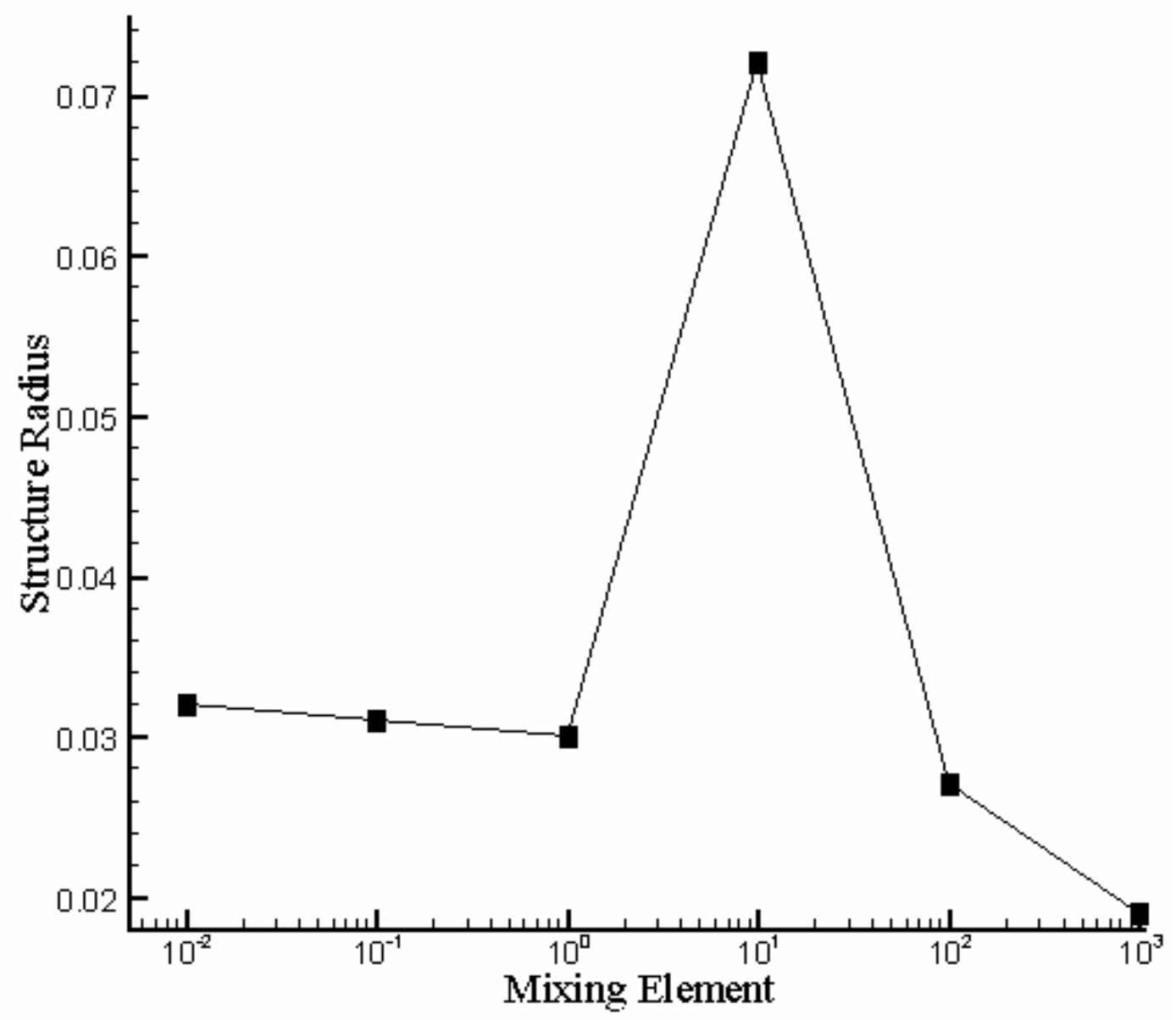

Figure 6.43: Structure radii at the end of the last mixing element (CMC concentration level: 5000 ppm) 


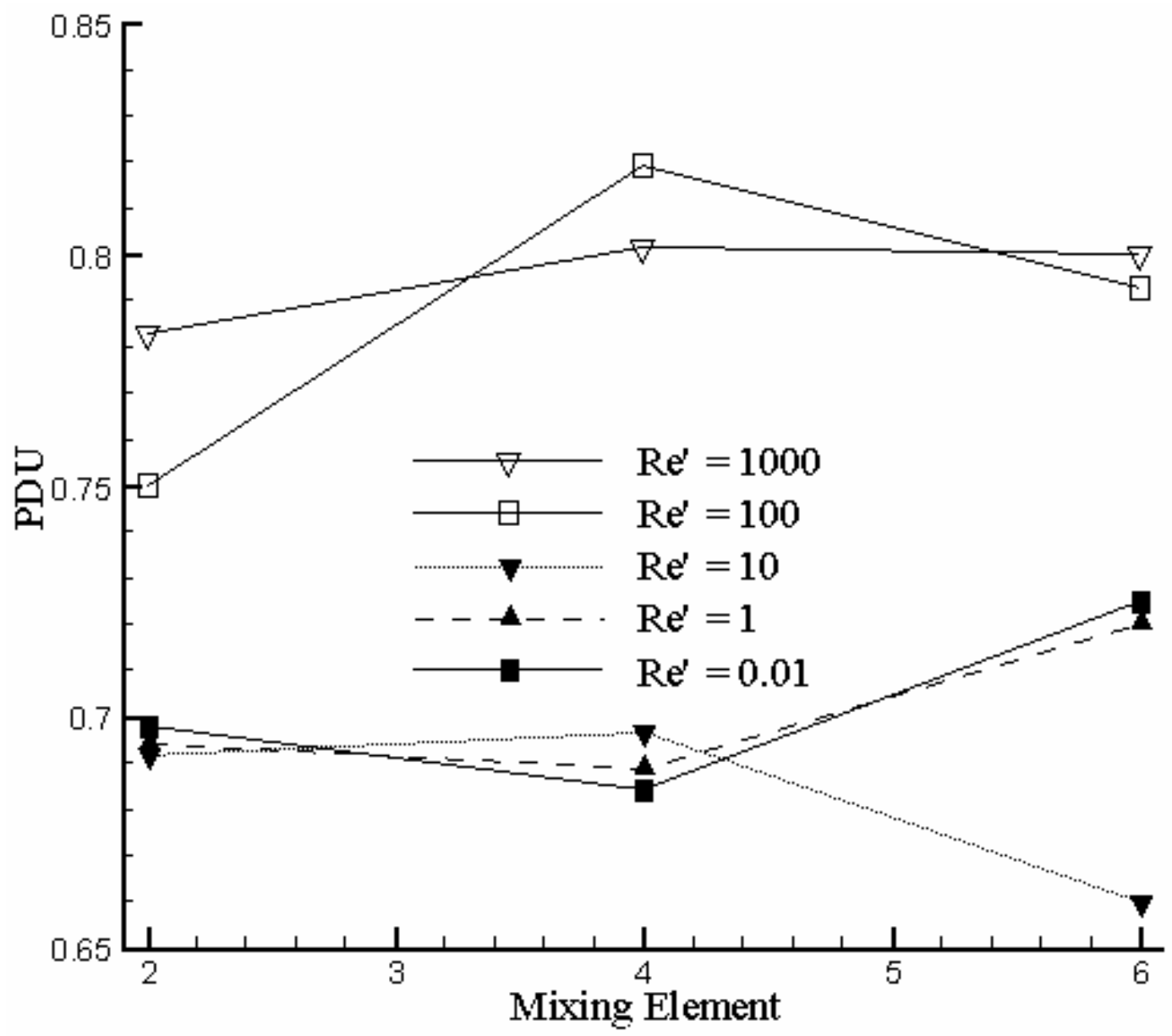

Figure 6.44: PDU values at the end of even numbered mixing elements (CMC concentration level: 50ppm) 


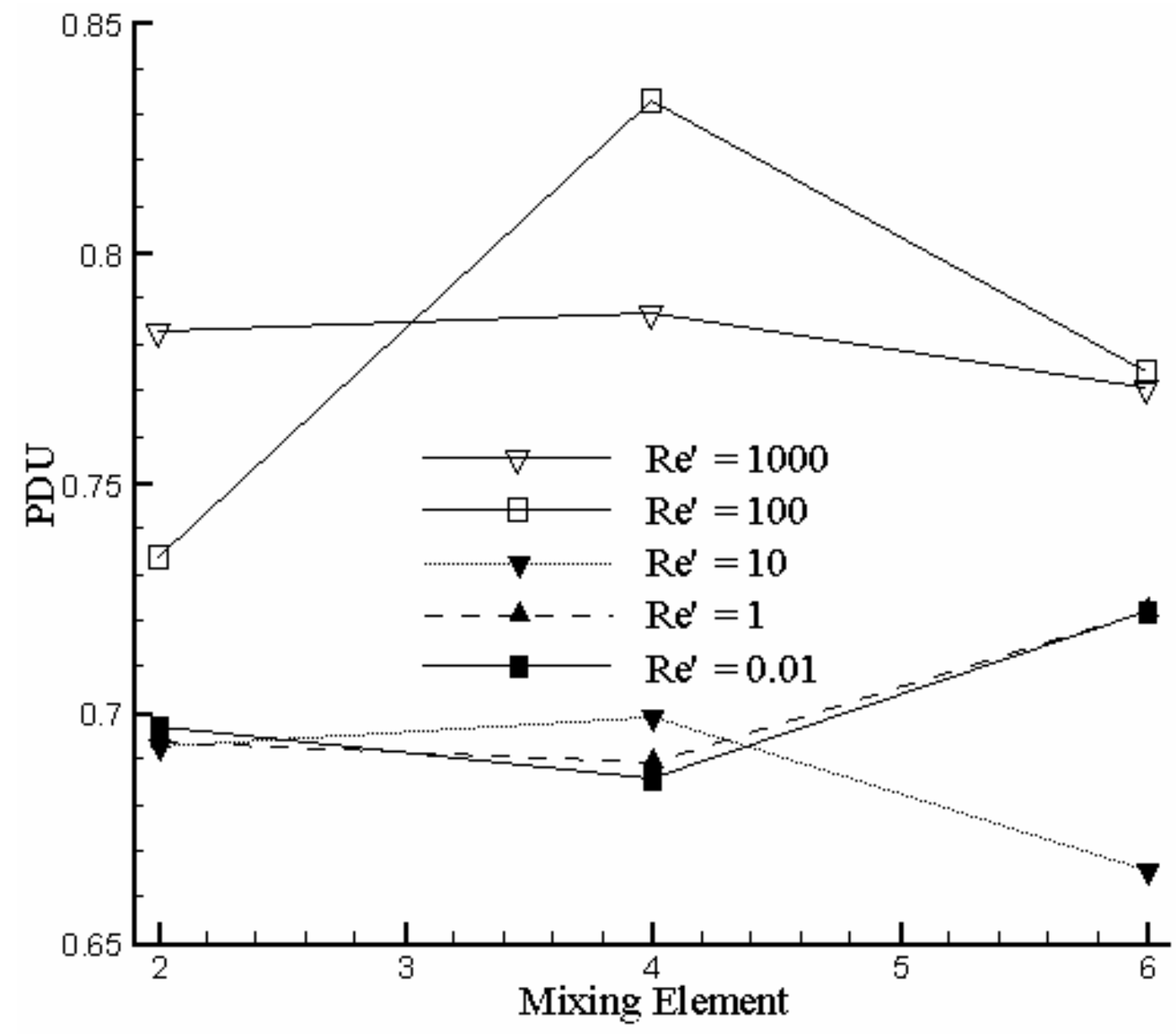

Figure 6.45: PDU values at the end of even numbered mixing elements (CMC concentration level: 500ppm) 


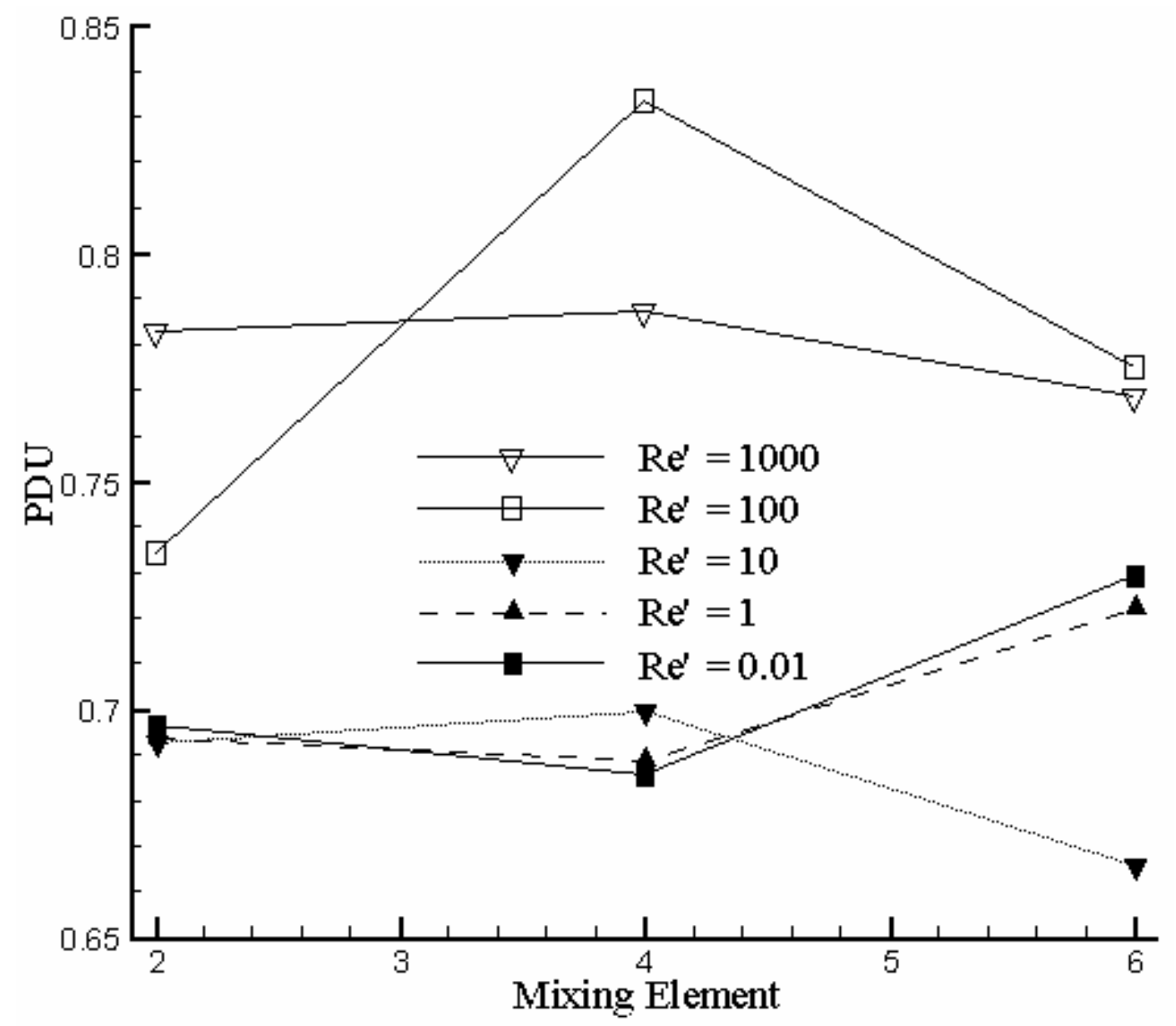

Figure 6.46: PDU values at the end of even numbered mixing elements (CMC concentration level: 5000ppm) 


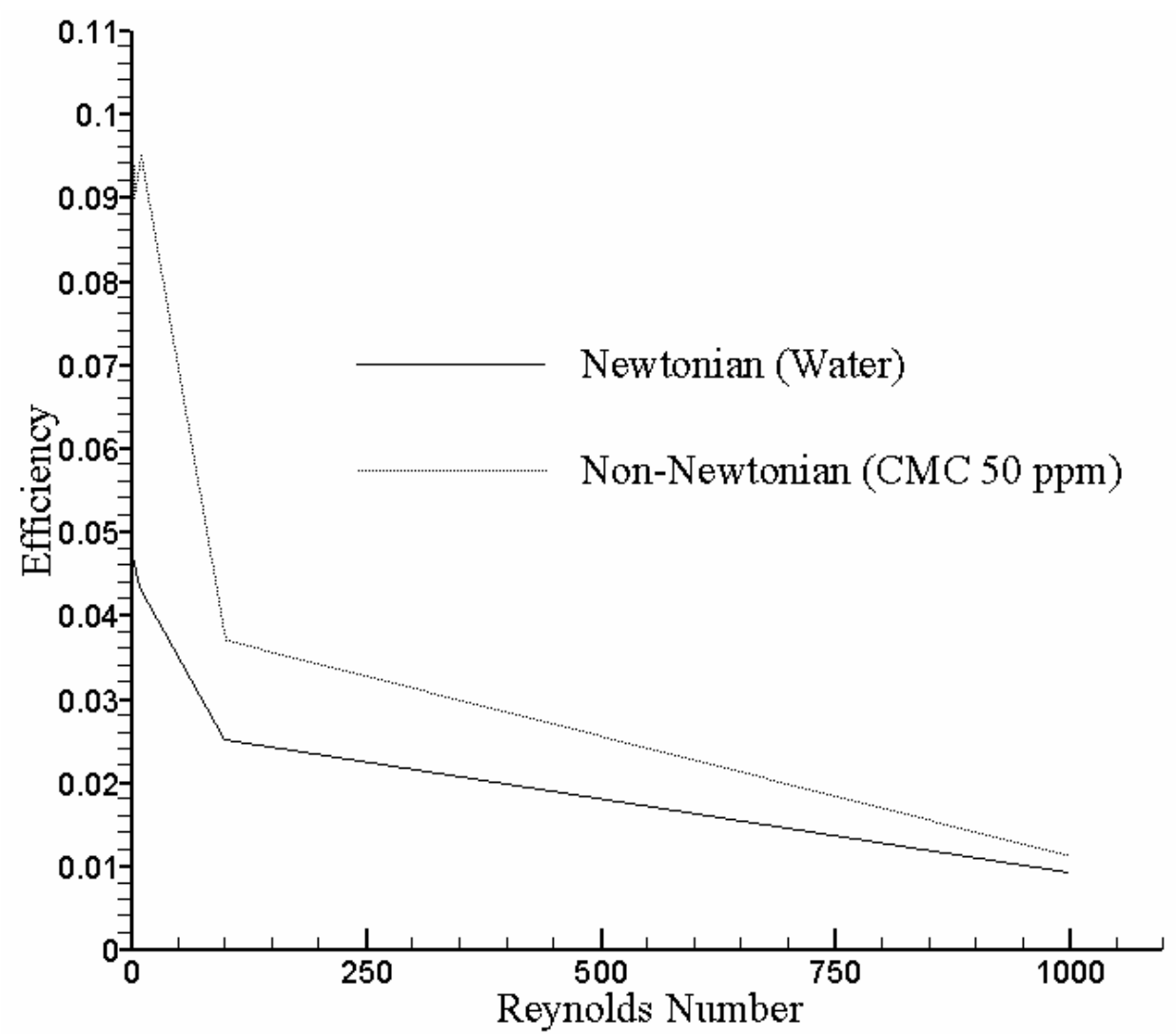

Figure 6.47: Helical static mixer efficiency 


\section{CHAPTER SEVEN}

\section{HELICAL STATIC MIXER PERFORMANCE UNDER TURBULENT FLOW REGIME CONDITIONS}

The usage of static mixers for turbulent flow conditions has increased within industry over recent years. Although the range of practical Reynolds numbers for helical static mixers in industrial applications is usually from very small $(\operatorname{Re} \sim 0)$ to moderate values $(\operatorname{Re} \sim 5,000)$, static mixers can be found in application with much higher Reynolds numbers $\left(\operatorname{Re}^{\sim} 100,000\right)$. It has been found that the flow regime in helical static mixers is turbulent for relatively low Reynolds numbers, compared to flow inside a pipe with no mixing elements present.

The most desirable approach to the numerical simulation of turbulent flows is by employing direct numerical simulations (DNS) methods. DNS does not involve approximations, other than those due to discretization, which are inherent in any numerical solution of differential equations, and so provides a benchmark against which less costly simulation methods may be compared. This is in contrast to using experimental data for the comparison. However, the continuum of different spatial (and temporal) scales intrinsic to turbulence, a continuum whose width increases with the Reynolds number, leads to computer time and storage requirements that increase rapidly 
with Re. As a consequence, DNS is limited to relatively low Reynolds numbers in practice and also to simple geometries; it is currently very expensive to conduct a DNS calculation at even moderately high Reynolds numbers. To undertake such simulations at the high Reynolds numbers typical of most industrial and naturally occurring flows is not feasible at present, nor is it likely to be in the near future. Calculations can be made to show that to resolve even a plane pipe flow problem of a moderate length, the number of numerical operations that are needed is of the order of $10^{22}$. This, with a machine with computational speed of $10 \mathrm{GHz}$, means that about 32,000 years of computer time is required, i.e., $\left(10^{22} / 10^{10}\right)(1 /(60 \times 60 \times 24 \times 365))=31,710$. Thus, as emphasized by Moin in [135], DNS is a research tool, rather than an aid to engineering design.

Recently some new approaches, for example non-linear eddy-viscosity (NLEVM) models, have been introduced to solve turbulent flow problems [136]. Despite substantial effort being expended on model validation, there continues to be much uncertainty on the predictive qualities of NLEVMs in complex flows [136,137]. In part, this is because a reliable assessment of models by reference to experimental data poses a number of problems unrelated to the models themselves and their numerical implementation. Issues of principal concern are (1) the compatibility of the computational implementation with the experimental conditions, (2) the completeness and accuracy of the boundary conditions and (3) the accuracy of the data used to assess the predictions obtained by the model. These uncertainties may be sufficiently serious to invalidate conclusions derived from a validation study. 
Two alternative methods can be employed to transform the Navier-Stokes equations in such a way that the small-scale turbulent fluctuations do not have to be directly simulated: Reynolds averaging and filtering. However, both methods introduce additional terms in the governing equations that need to be modeled in order to achieve closure. In the first approach, Reynolds-averaged, Navier-Stokes equations are solved. In the second approach, the large scale turbulent motion is computed and the small scale turbulent motion is modeled [138-140].

This chapter deals with the prediction of turbulent flow in a six-element helical static mixer. Most common Reynolds-Averaged Navier-Stocks (RANS) turbulence models and also Large-Eddy Simulation (LES) are used to study the static mixer performance under turbulent flow conditions.

\subsection{RANS Turbulent Models}

Selecting a suitable turbulence model is an important step in the numerical study of a fully three-dimensional flow of an industrial device. In this section, some of the most common Reynolds averaged Navier-Stokes (RANS) turbulence models are employed in order to simulate the flow in an industrial helical static mixer. The global performance of these models are compared in order to select the most suitable and practical model in order to design/redesign and study the performance of a mixer.

Turbulent flows represent an intrinsically three-dimensional, time and space dependent phenomena, which have a large number of spatial degrees of freedom. Turbulent flows have a wide range of vorticities from large- to small-scale, which have a 
random nature. Turbulent flows are characterized by fluctuating velocity fields. These fluctuations mix transported quantities of mass, momentum, and energy and in turn cause the transported quantities to fluctuate as well. A complete time-dependent solution of the exact Navier-Stokes equations for turbulent flows in complex geometries is too expensive and in most cases impractical. Two alternative methods have been employed to transform the Navier-Stokes equations in such a way that the small-scale turbulent fluctuations do not have to be directly simulated: Reynolds averaging and filtering. However, both methods introduce additional terms in the governing equations that need to be modeled.

The Reynolds-averaged Navier-Stokes (RANS) equations represent transport equations for the mean flow quantities; and all the scales of the turbulence are modeled. This approach significantly reduces the computational effort; and therefore, the use of RANS models is preferred for practical engineering calculations.

The three-dimensional, steady Reynolds-averaged, Navier-Stokes equations are to be solved. The momentum equation can be written as:

$$
\rho \frac{\partial\left(u_{i} u_{j}\right)}{\partial x_{i}}=\frac{\partial}{\partial x_{j}}\left[\mu\left(\frac{\partial u_{i}}{\partial x_{j}}+\frac{\partial u_{j}}{\partial x_{i}}\right)-\frac{2}{3} \mu \frac{\partial u_{k}}{\partial x_{k}} \delta_{i j}\right]-\frac{\partial p}{\partial x_{i}}+\frac{\partial}{\partial x_{j}}\left(-\rho \overline{u_{i}^{\prime} u_{j}^{\prime}}\right)
$$

Considering the conservation of mass for an incompressible steady flow, $\partial u_{k} / \partial x_{k}=0$; hence, Eq. (7.1) becomes: 


$$
\rho \frac{\partial\left(u_{i} u_{j}\right)}{\partial x_{i}}=\frac{\partial}{\partial x_{j}}\left[\mu\left(\frac{\partial u_{i}}{\partial x_{j}}+\frac{\partial u_{j}}{\partial x_{i}}\right)\right]-\frac{\partial p}{\partial x_{i}}+\frac{\partial}{\partial x_{j}}\left(-\rho \overline{u_{i}^{\prime} u_{j}^{\prime}}\right)
$$

And when the fluid viscosity is constant, Eq. (7.2) reduces to the following:

$$
\rho \frac{\partial\left(u_{i} u_{j}\right)}{\partial x_{i}}=\mu \frac{\partial}{\partial x_{j}}\left(\frac{\partial u_{i}}{\partial x_{j}}+\frac{\partial u_{j}}{\partial x_{i}}\right)-\frac{\partial p}{\partial x_{i}}+\frac{\partial}{\partial x_{j}}\left(-\rho \overline{u_{i}^{\prime} u_{j}^{\prime}}\right)
$$

The Reynolds stresses, $\left(-\rho \overline{u_{i}^{\prime} u_{j}^{\prime}}\right)$, must be modeled in order to close the set of equations. An ideal model should introduce the minimum amount of complexity while capturing the essence of the relevant physics.

The earliest attempts at developing a mathematical description of turbulent stresses sought to mimic the molecular gradient-diffusion process. In this spirit, Boussinesq (1877) introduced the concept of an eddy viscosity. As with Reynolds, Boussinesq has been immortalized in turbulence literature. In turbulence models that employ the Boussinesq approach, the central issue is how the eddy viscosity is computed.

Spalart-Allmaras Model: The model proposed by Spalart and Allmaras [100] solves a transport equation for a quantity that is a modified form of the turbulent kinematic viscosity. In these models a transport equation is solved for a turbulent quantity (usually the turbulent kinetic energy) and a second turbulent quantity (usually a turbulent length scale) is obtained from an algebraic expression. The turbulent viscosity is calculated from 
the Boussinesq assumption. This model is incomplete however as it relates the turbulence length scale to some typical flow dimension.

The Spalart-Allmaras model is a relatively simple one-equation model that solves a modeled transport equation for the kinematic eddy viscosity. This embodies a relatively new class of one-equation models in which it is not necessary to calculate a length scale related to the local shear layer thickness. The Spalart-Allmaras model was designed specifically for aerospace applications involving wall-bounded flows and has been shown to give good results for boundary layers subjected to adverse pressure gradients. It is also gaining popularity in turbomachinery applications, e.g., as described in [141]. This model is effectively a low-Reynolds-number model, requiring the viscous-affected region of the boundary layer to be properly resolved.

$\boldsymbol{k}$-? Model: Based on the Boussinesq hypothesis, Kolmogorov [91] introduced the first complete model of turbulence. In addition to having a modeled equation for the kinetic energy of the turbulent fluctuations, $k$ [90], Kolmogorov introduced a second parameter $\omega$ that he referred to as the rate of dissipation of energy per unit volume and time. The reciprocal of $\omega$ serves as a turbulence time scale, while $\sqrt{k} / \omega$ serves as the analog of the mixing length. The $k-\omega$ model has been validated extensively in complex, threedimensional shear flows $[142,143]$.

The $k-\omega$ model used in this study has been introduced by Menter in 1994 [109], which is a modified version of the original model of Wilcox [103]. One problem with the original model is that it does not correctly predict the asymptotic behavior of the turbulence in near-wall regions, because of the fact that the eddy viscosity is much 
smaller than the molecular viscosity close to a solid surface [109]. Menter developed two additional versions of the $k-\omega$ model. The first model utilized Wilcox's $k-\omega$ model in the inner region of the boundary layer and switches $\dot{i}$ to the standard version of $k-\varepsilon$ model in the outer region and in free shear flows. The second model, referred to as ShearStress Transport model or the SST $k-\omega$ model, is a modification of the first model. This model is based on the philosophy underlying the Johnson-King model [144], which holds that the transport of the principal turbulent shear stress is of vital importance in the prediction of severe adverse pressure gradient flows. This model does not consume much more computing time compared to the original version [109]. The $k-\omega$ model used in this study is the SST $k-\omega$ model.

$\boldsymbol{k}$-e Model: By far, the most extensive work on two-equation models has been done by Launder and Spalding [101] and a continuing succession of students and colleagues. Launder's $k-\varepsilon$ model, where $\varepsilon$ is proportional to the product of $k$ and $\omega$, is as well known as the mixing-length model and is the most widely used two-equation model. Even the model's demonstrable inadequacy for flows with an adverse pressure gradient [102] has done little to discourage its widespread use. In this study the standard $k-\varepsilon$ model is employed to simulate flow in a static mixer, because of its robustness and reasonable accuracy for a wide range of industrial turbulent flow simulations.

It should mentioned that using the Spalart-Allmaras model, the near-wall gradients of the transported variable in this model are much smaller than the gradients of the transported variables in the $k-\varepsilon$ and $k-\omega$ models. This might make the model less sensitive to numerical error when non-layered meshes are used near walls. The original 
model is based on the idea that, for the wall-bounded flows that were of most interest when the model was formulated, turbulence is found only where vorticity is generated near walls. However, it has since been acknowledged that one should also take into account the effect of mean strain on the turbulence production, and a modification to the model has been proposed by Dacles-Mariani, et al., [145], which combines measures of both rotation and strain tensors. In fact, Dacles-Mariani, et al., introduced a modification to the Baldwin-Barth [98] turbulence model, which is based on the idea previously proposed by Spalart $[100,145]$. Including both the rotation and strain tensors reduces the production of eddy viscosity and consequently reduces the eddy viscosity itself in regions where the measure of vorticity exceeds that of strain rate. One such example can be found in vortical flows, i.e., flow near the core of a vortex subjected to a pure rotation where turbulence is known to be suppressed. Including both the rotation and strain tensors more correctly accounts for the effects of rotation on turbulence. The default option (including the rotation tensor only) tends to over-predict the production of eddy viscosity and hence over-predicts the eddy viscosity itself in certain circumstances.

RSM Model: An alternative to the Boussinesq hypothesis, which relates the Reynolds stresses to the mean velocity gradients, is to solve the transport equations for each of the terms in the Reynolds stress tensor, known as Reynolds Stress Model (RSM). The RSM model involves calculation of the individual Reynolds stresses, using differential transport equations $[113,146]$. The individual Reynolds stresses are then used to obtain closure of the Reynolds-averaged momentum equation. The exact form of the Reynolds stress transport equations may be derived by taking moments of the momentum equation. 
This is a process wherein the complete momentum equations are multiplied by a fluctuating property, the product then being Reynolds-averaged.

The field of classical RANS turbulence modeling is dynamic. The full range of range of RANS models continues to be researched, which testifies that no class of models has emerged as clearly superior or clearly hopeless [147]. However, it happens that the Spalart-Allmaras model, the SST $k-\omega$ model and the $k-\varepsilon$ model, all three of which are insensitive to free-stream values, and their results are reproducible from code to code and grid to grid, can be described as popular turbulence models [147].

Whenever non-isotropic effects are important, use of the RSM model should be considered. Note that in a turbulent boundary layer the turbulence is always nonisotropic, but isotropic eddy viscosity models, such as $k-\omega$ and $k-\varepsilon$ models, handle this type of flow excellently as far as mean flow quantities are concerned. On the other hand, a $k-\varepsilon$ model gives very poor representation of the normal stresses. In flows with strong curvature and swirling flows, non-isotropic effects often are important; this is the type of flow that occurs in a helical static mixer. Therefore, the RSM has also been selected to predict the flow properties in the static mixer.

The $k-\varepsilon$ model and the RSM model are primarily valid for the turbulent core. Consideration therefore needs to be given as to how to make these models suitable for wall-bounded flows. One common approach to modeling the near-wall region is using semi-empirical formulas (wall functions) to bridge the viscosity-affected region between the wall and the fully-turbulent region. The use of wall functions eliminates the need to modify the turbulence models to account for the presence of the wall. The wall function 
proposed by Launder and Spalding [148], usually called the standard wall function has been most widely used for industrial flows. However, the standard wall function displays poor behavior when the flow is subjected to a pressure gradient in the near wall region. Kim and Choudhury [149] introduced a two-layer-based, non-equilibrium wall function which is more suitable for such a flow. It has been applied to a number of complex flows involving separation, recirculation and reattachment and showed substantial improvement compared to the standard wall function. Employing the wall function approach substantially saves computational resources in most cases and is a practical option for the near-wall treatments for industrial flow simulations.

However, it should be mentioned that turbulence models that rely on the wallfunction approach, although expedient from a computational standpoint are not suitable for quantitatively accurate predictions of complex, three-dimensional flows with strong vortices [150]. The use of a wall function decreases the accuracy of the turbulence model prediction in low-Reynolds-number flows, in flows with a high pressure gradient near the wall region, and in strongly skewed three-dimensional flows. In this study, for the RSM model, an enhanced wall treatment is employed, and therefore, the viscosity-affected region near the solid wall is entirely resolved all the way to the viscous sublayer. In order to be able to resolve the laminar sublayer the computational grid in the near wall region needs to be fine enough. The enhanced wall treatment, used in this study, is based on the work of Chen and Patel [151] and on the model introduced by Wolfstein [152], which is used to determine the turbulent viscosity in the viscous sublayer region. A blending function is used in order to extend the applicability of the method throughout the laminar 
sublayer, the fully-turbulent region, and the buffer region between the sublayer and the fully-turbulent outer region. For the $k-\varepsilon$ model, both the non-equilibrium wall function and the enhanced wall treatment are used to simulate the flow around a static mixer. The results obtained by this approach are compared.

\subsubsection{Numerical Approach}

The flow solver uses the Eulerian approach to solve the equations on a computational mesh, based on a cell-centered finite volume discretization. The selected solver uses segregated, implicit, and upwind [119] methods. Also all calculations were performed in double precision. Pressure-velocity coupling is achieved by using the SIMPLEC (SIMPLE-Consistent) algorithm [121].

No-slip boundary conditions are applied to the solid surface of the static mixer and also at the wall of the pipe. A constant mass flow rate is applied at the inlet and outlet boundaries. Fully developed flow conditions are assumed at the inlet; the one seventhroot law is used for the velocity profile at the flow field inlet. The turbulence intensity is used to specify the turbulent boundary conditions at the flow inlet. The turbulence intensity for a fully-developed duct flow can be estimated from an empirical correlation for pipe flows [153]. The turbulence intensity, for the geometry used here, is equal to $6.99 \%$ for the case of $\operatorname{Re}=3,000$ and it is $6.56 \%$ for $\operatorname{Re}=5,000$.

For a steady-state laminar flow, the particle trajectories correspond to streamlines and therefore, trajectories are tracked by integrating the vector equation of motion, using the numerically computed velocity field as input. However, for turbulent flows, some 
considerations in using the results of a steady RANS calculation are needed. In turbulent flows scalar transport and stirring is the result of both by the large-scale coherent vortices and the broad range of turbulent eddies. RANS modeling procedure smoothes flowfield and therefore, particle tracking using the statistically stationary mean flow as the advecting velocity field does not take into account the effect of small scale turbulence on transport. In order to overcome this shortcoming, turbulent Reynolds stresses can be used to generate instantaneous velocity. In RANS turbulence modeling approach information about turbulent fluctuations is contained in the time averaged Reynolds stresses of the form $\overline{u_{i}^{\prime} u_{j}^{\prime}}$. Stochastic techniques can be used in order to generate a random fluctuating velocity field using the turbulence intensities. These fluctuations are superimposed to the calculated time-averaged velocity field and create an instantaneous velocity fields. Fluid particle tracking is carried out using this instantaneous flowfield. A considerable amount of work in random flow generation using RANS model can be found in the literature, e.g. [154-158]; however, in some of these works, the generated flowfield does not satisfy the requirement of spatial inhomogeneity and anisotropy of turbulent shear stresses or the generated flowfield does not satisfy the continuity conservation law [159]. For the results presented in this thesis, a random flow generation technique presented by Smirnov et. al [159] is employed. The method is a modified version of Kraichnan's technique [160] and is able to generate a realistic instantaneous flowfield efficiently. 


\subsubsection{Numerical Solution Accuracy}

It is important that the accuracy of the numerical solutions be analyzed before confidence in the predictive ability of the numerical techniques can be justified. As mentioned previously, for the present study of mixing under non-creeping flow conditions, there is unfortunately an absence of experimental data that is sufficiently detailed and accurate to undertake a complete validation of the numerical results. A detailed mesh convergence study has indicated that the computational mesh employed in the present study is sufficiently refined to provide good numerical resolution. Furthermore, the computed pressure drop in the pipe containing the mixer elements is contrasted to the measured data. Also, the velocity contours at the computational flow field outlet are considered. As a quantitative measure of the fluid mixing, the size of the structures at the flow field outlet is considered. Figure 7.1 shows the velocity (left) and vorticity (right) contours for $\operatorname{Re}=5,000$, predicted by different grids with $1,478,334$, $2,086,955$, and 2,822,681cells, respectively from top to bottom. The pressure drop across the mixer predicted using grids containing 1,478,334 and 2,086,955 cells are $91.19 \%$ and $98.33 \%$ of the pressure drop predicted using a mesh with 2,822,681cells, 2,086,955. The obtained results do not change noticeably after that. Table 7.1 gives the information related to the computational grid used in this study for the Spalart-Allmaras, the $k-\varepsilon$ (using enhanced wall treatment), the $k-\omega$, and the RSM turbulence models.

For the particle tracking studies, different numbers of particles (from 31,180 particles up to $1,225,784$ particles) were used to analyze the mixing. By increasing the number of particles, it is possible to discern finer structures and thus quantify the mixing efficiency 
with higher precision. Tracking of only a relatively small number of particles, released at the flow field inlet, gives the impression that a high level of mixing has been achieved at the exit of the mixer. As this number increases, the presence of the striations associated with the mixing process is more and more evident. It was observed that 501,740 particles at the flow inlet can give a correct image of mixing; although using a higher number of particles leads to a more pronounced mixing pattern, but the difference between the obtained results is not that significant to justify the computational cost needed for calculations.

Comparison with existing experimental values of the pressure drop across the mixer measured and also vorticity and velocity contours studies at different flowfield crosssection show that the computational grid is fine enough to predict numerical values accurately.

\subsection{Results and Discussion Using RANS Models}

Using the turbulence modeling techniques described above, the flow in a six-element static mixer has been analyzed. In each case the initial conditions are set so that the axial velocity is equal to the bulk velocity and the two other velocity components are set to

zero. As the convergence criterion, a scaled residual [123] less than $10^{-5}$ for all scalar equations, is used here. Table 7.2 shows the values of pressure drop across the mixer, area-weighted average velocities, and area-weighted average vorticity at the end of the sixth mixing element, non-dimensionalized by the values as predicted from the converged 
solution, for the case of the $k-\omega$ model, when $\operatorname{Re}=3,000$. The results obtained were found to vary negligibly once this condition was reached.

Table 7.3 presents a comparison of the number of iterations, the required CPU time per iteration and the computational memory needed for the different turbulence models, using the same computational grid. The CPU time for each model is nondimensionalized by the CPU time needed for one iteration using the $k-\omega$ turbulence model and the computational memory is also nondimensionalized by the memory needed using the $k-\omega$ model. The least expensive and the most expensive models are the $k-\varepsilon$ model and the RSM model, respectively. The total CPU time for the $k-\varepsilon$ and the RSM models is 0.52 times and 1.64 times of the total CPU time using the $k-\omega$ model, respectively.

The velocity fields for each turbulence model were obtained. The cross-sectional velocity vectors are illustrated in Figs. 7.2-7.5, which show the cross-sectional projection of the velocity vectors at the end of the second, the fourth, and the sixth elements, for $\mathrm{Re}=3,000$. Contours of velocity magnitude are shown in Figs. 7.6-7.9. The velocity field predicted by the Spalart-Allmaras and $k-\omega$ models are quiet similar. The pattern of the velocity field predicted by the $k-\varepsilon$ model is relatively close to the one predicted by the RSM model. The main difference between the velocity field predicted by the SpalartAllmaras and the $k-\omega$ models and the one predicted by the $k-\varepsilon$ and the RSM models is that the first two models are able to capture more curvature of the velocity; these models presents a more detailed velocity field. This can be explained by considering the fact that the Spalart-Allmaras and the $k-\omega$ models were designed for applications involving wall-bounded flows and low Reynolds number regions. On the other hand, the 
standard $k-\varepsilon$ model is designed for high Reynolds turbulent flows. The $k-\varepsilon$ and the RSM models are designed for turbulent flows in regions somewhat far from walls. Therefore, for the flow considered here, which is surrounded by solid walls and filled by low Reynolds number regions, turbulence models that rely on wall treatments are not able to predict a very accurate and detailed velocity field.

The trajectories of the particles injected into the mixer from the top half on flow inlet have been calculated. As the number of the mixing elements increases, the number of lost particles also increases. The total number of particles released into the flow field is 501,740 . At most $7.29 \%, 6.74 \%, 7.10 \%$, and $6.58 \%$ of the particles are lost by using the Spalart-Allmaras, the $k-\varepsilon$, the $k-\omega$ and the RSM models, respectively. The ratio of the lost particles at the end of the sixth mixing element to the lost particles at the end of the second element is $2.4096,2.5223,2.4862$, and 2.3739, for the Spalart-Allmaras, the $k-\varepsilon$, the $k-\omega$ and the RSM models, respectively.

The plots of the particle positions at the end of the second, the fourth, and the sixth elements, are shown respectively from left to right, in Figs. 7.10-7.13, for $\operatorname{Re}=3,000$. These plots illustrate the redistribution of the released particles via the combined effects of flow division and reversal, resulting in stretching and folding of the observed structures. Comparing the results of each model at different cross-sections reveals that islands of separated fluid elements are distinguishable after the flow passes the second mixing element. These islands are divided into several small regions after the flow passes the fourth element; the level of darkness in the corresponding figures decreased, suggesting that two fluids are penetrating each other. At the sixth element, the large 
portion of field is filled with gray regions and the particles are distributed more uniformly in the mixer cross-section; the area of the flow cross-section containing no particles is much smaller.

The turbulence intensity in the flow inlet has a major impact on the particles trajectory and therefore on the mixing of the working fluids at the first mixing elements. It was observed that as the flow passes through more mixing elements the distribution of the particles is less affected by the turbulence intensity at the flow inlet. Moreover, at the sixth mixing element this effect has almost vanished.

On the other hand, comparing the numerical results from the different turbulence models at the same cross-section for the same Reynolds number shows that each model predicts a different particle distribution; however, the overall patterns obtained by the Spalart-Allmaras, the $k-\omega$, and the RSM models are similar. The particle distribution predicted by the $k-\varepsilon$ model prediction is significantly different from those predicted by the other three models. This can be explained by considering the vorticity values predicted by these models. The mean and the maximum vorticity magnitudes at these mixing elements are presented in Table 7.4. As can be seen, the $k-\varepsilon$ predicts lower values of vorticity. A small difference in the velocity leads to significant differences in the vorticity values. An underestimation of the vorticity distribution leads to the underestimation of flow mixing.

A more accurate turbulence model, as well as a more accurate discretization method, can capture more physics of the problem and provide a more detailed solution. Therefore, even for fluid particles released very near each other, distinct paths are detectable. This 
feature is not achievable, using less accurate turbulence models, due to their inability to resolve small vortical structures. In fact, it was observed, in practice, that using less accurate turbulence models, fluid particles released from neighboring points follow virtually indistinguishable pathlines. This can explain why in the obtained results by the $k-\varepsilon$ model, fluid particles are shown less distributed in different flow cross-sections compared to the results obtained by the other turbulence models.

Determining the pressure drop across a static mixer is essential in order to correctly size the extruder or pump, feeding the mixer. The predicted pressure drop through the mixer by different models is shown in Table 7.5. The experimentally measured pressure drop through the mixer is $1,180 \mathrm{~Pa}$. All turbulence models are able to predict the pressure drop accurately. The least accurate prediction was made by the $k-\varepsilon$ model, which underestimates the experimental pressure drop by about 4.3\%. The RSM model overestimates the pressure drop by about $0.5 \%$.

Table 7.6 presents the calculated G-values based on the predicted pressure drop across mixer by different turbulence models. All these models predict almost the same Gvalue for the helical static mixer studied here.

Values of the structure radius at different flow cross-sections are given in Table 7.7. Different turbulence models predict different values for the structure radius at a certain flow cross-section. However, the differences between the predicted values are not significant.

As expected, the structure radius decreases as the flow passes through the static mixer. The rate of change of the structure radius from the flow cross-section at the end of 
the second element to the end of the fourth element is higher that the one from the flow cross-section at the end of the fourth element to the end of the sixth element. This suggests that upstream mixing elements increase the mixing more efficiently compared to the downstream mixing elements.

Figures 7.14-7.17 show the distribution function for flow across a six-element helical static mixer inside a pipe, using different turbulence models. The non-dimensional time step, $d t^{*}$, is 0.01 . The maximum nondimensionalized residence time, $t^{*}$, predicted by the $k-\varepsilon$ and the RSM models is about 1.6; however, the $k-\omega$ and the Spalart-Allmaras models predict this value about 2.3. In the results of these models two modes are distinguishable, the first mode starting at about $t^{*}=0.5$ and the second mode starting at about $t^{*}=1.4$. The second mode is substantially weaker than the first mode. All four turbulence models are able to predict the first mode. The first mode is associated with streamlines that occupy a large portion of the flow. All these models predict similar pattern for distribution function in its first mode.

Table 7.8 shows values of the standard deviation of the distribution function, based on the velocity domain predicted by different turbulent models. All the values are from the same order of magnitude, although the $k-\varepsilon$ predicts a standard deviation of distribution function which is less than $70 \%$ of the standard deviations predicted by other models.

For the $k-\varepsilon$ model, both the non-equilibrium wall function and the enhanced wall treatment were employed to study the flow in static mixer. Using non-equilibrium wall function, the size of computational grid needed for the $k-\varepsilon$ model is only $8.18 \%$ of the 
size of the grid using the $k-\varepsilon$ model with the enhanced wall treatment; and the iterations needed to obtain a converged solution is $32.88 \%$. The pressure drop predicted by the $k-\varepsilon$ model, using a wall function, is 0.9805 times the pressure drop predicted by this model, using the enhanced wall treatment. The area-weighted average velocity and the maximum velocity at the flow outlet predicted by the $k-\varepsilon$ model, using a wall function, are 0.8926 and 0.9225 times the pressure drop predicted by this model, using the enhanced wall treatment, respectively. However, for the area-weighted average vorticity and the maximum vorticity at the flow outlet, these ratios are 0.6936 and 0.1466 , respectively. Therefore, it is possible to use the wall function in order to obtain rough estimations about the bulk values, such as pressure drop. However, wall functions should not be used if a detailed study of the performance of the mixer is intended.

The SST $k-\omega$ and the RSM models were also employed in order to predict the flowfield in a pipe containing a six-element helical static mixer, when $\operatorname{Re}=5,000$. The $k-\omega$ model overestimates the pressure drop by $4.18 \%$, and the RSM model overestimates the pressure drop by $1.75 \%$. The maximum difference in predicted velocities, by these models, is $6.37 \%$. By increasing the flow Reynolds number from 3,000 to 5,000, the G-value increases about 2.12 times. The required energy to maintain the flow through a helical static mixer, in a turbulent flow regime, is significantly increased, when the flow rate increases. 


\subsection{Large-Eddy Simulation}

Turbulent flows are characterized by eddies with a wide range of length and time scales. The largest eddies are typically comparable in size to the characteristic length of the mean flow. The smallest scales are responsible for the dissipation of turbulence kinetic energy. Large eddies are more problem-dependent. They are dictated by the geometries and boundary conditions of the flow involved; small eddies are less dependent on the geometry, tend to be more isotropic, and are consequently more universal. Basically in a large-eddy simulation (LES) large eddies are resolved directly, while small eddies are modeled.

LES uses a set of filtered equations. Filtering is essentially a manipulation of the exact Navier-Stokes equations to remove only eddies that are smaller than the size of the filter, which is usually taken as the mesh size. The filtering process creates additional unknown terms that must be modeled in order to achieve closure. Statistics of the mean flow quantities, which are generally of most engineering interest, are gathered during the time-dependent simulation. The attraction of LES is that, by modeling less of the turbulence (and solving more), the error induced by the turbulence model will be reduced.

Solving only for the large eddies and modeling the smaller scales results in mesh resolution requirements that are much less restrictive than those of a DNS simulation. Typically, mesh sizes can be at least one order of magnitude smaller than those required by DNS. Furthermore, the time step sizes will be proportional to the eddy-turnover time, which is much less restrictive than that with DNS. Conceptually, LES is situated 
somewhere between DNS and the Reynolds-averaged Navier-Stokes approach. However, it should be stressed that the application of LES to industrial flow simulations is in its infancy [161]. Here, a Renormalization group based LES turbulent model [140] is used to study turbulent flow across a static mixer; and the results can also be regarded as validation data to study the accuracy of common engineering turbulent models on complex geometries.

The governing equations employed by LES are obtained by filtering the timedependent Navier-Stokes equations in either Fourier (wave-number) space or configuration (physical) space. Filtering the incompressible Navier-Stokes equations, one obtains

$$
\frac{\partial \bar{u}_{i}}{\partial x_{i}}=0
$$

and

$$
\rho \frac{\partial \bar{u}_{i}}{\partial t}+\rho \frac{\partial\left(\bar{u}_{i} \bar{u}_{j}\right)}{\partial x_{j}}+\frac{\partial \bar{p}}{\partial x_{i}}+\frac{\partial \hat{\tau}_{i j}}{\partial x_{j}}-\frac{\partial\left(\mu \frac{\partial \bar{u}_{i}}{\partial x_{j}}\right)}{\partial x_{i}}=0
$$

where $\hat{\tau}_{i j}$ is the subgrid-scale stress defined by

$$
\hat{\tau}_{i j}=\rho \overline{u_{i} u_{j}}-\rho \bar{u}_{i} \bar{u}_{j}
$$


The subgrid-scale stresses resulting from the filtering operation are unknown, and require modeling. While subgrid-scale modeling and near-wall resolution are, generally, issues that affect accuracy, the use of sufficiently fine grids can be made to render both practically insignificant, at least in terms of the present exploitation of the data for model validation.

One common approach is the use of eddy viscosity models, which may be written in the following form [162]

$$
\hat{\tau}_{i j}-\frac{\delta_{i j}}{3} \hat{\tau}_{k k}=-2 \mu_{t} \bar{S}_{i j}
$$

where $\mu_{t}$ is the subgrid-scale turbulent viscosity, and $\bar{S}_{i j}$ is the rate-of-strain tensor for the resolved scale defined by

$$
\bar{S}_{i j}=\frac{1}{2}\left(\frac{\partial \bar{u}_{i}}{\partial x_{j}}+\frac{\partial \bar{u}_{j}}{\partial x_{i}}\right)
$$

In this study, Renormalization group (RNG) theory is used to derive a model for the subgrid-scale eddy viscosity [140]. The RNG procedure results in an effective subgrid viscosity, $\mu_{\text {eff }}$, given by 


$$
\mu_{e f f}=\mu+\mu_{t}=\mu\left(1+H\left(\frac{\mu_{s}^{2} \mu_{e f f}}{\mu^{3}}-C_{1}\right)\right)^{\frac{1}{3}}
$$

where $\mathrm{H}$ is the Heaviside function, and $\mu_{s}$ is given by

$$
\mu_{s}=C_{2} v^{\frac{2}{3}} \sqrt{\bar{S}_{i j} \bar{S}_{i j}}
$$

For theoretical reasons, the constants $C_{1}$ and $C_{2}$ in Eqs. (7.9) and (7.10) have the following values: $C_{1}=100, C_{2}=0.034859$ [140].

In problems containing regions of low Reynolds number flow, the argument of the ramp function becomes negative and the effective viscosity reverts to the molecular viscosity. This enables the RNG based subgrid-scale eddy viscosity to model the lowReynolds-number effects encountered in transitional flows and near-wall regions.

\subsubsection{Numerical Approach}

An unstructured hexagonal grid was generated to model the six-element static mixer inside a pipe; the geometry was modeled completely, with no simplifications. To achieve the most accurate results of the solver all efforts have been taken to maximize the quality of the grid. For the computational grid here, the deviation from orthogonality is indistinguishable; the grid has a very high quality. A computational grid with $3,756,070$ 
cells has been generated and used. Table 7.9 shows the results of different me sh quality tests performed on the generated grid, used in this study.

Although, there are no computational restrictions on the near-wall mesh spacing, for the LES model, for best results, it was found be necessary to use very fine near-wall mesh spacing. Here, $y^{+} \leq 0.997$ for wall-boundary cells.

The flow parameters are initially computed using the $k-\omega$ turbulent flow model. A steady state, segregated, implicit, upwind [119], finite volume solver using double precision is employed to obtain primary results. These base results are then used as initial conditions for the LES model for turbulence. An unsteady second-order implicit time marching approach was employed besides a central differencing spatial discretization to compute the flow field in the mixer. Pressure-velocity coupling is achieved by using the SIMPLEC (SIMPLE-Consistent) algorithm [121].

No-slip boundary conditions are applied to the solid surface of the static mixer and also at the wall of the pipe. Constant mass flow rate is applied to the inlet and outlet boundaries. Different Reynolds numbers for the flow inside the pipe are obtained by varying the mass flow rate at the inlet boundary. Flow conditions at the inlet are assumed fully developed. The turbulence intensity is used to specify the turbulent boundary conditions at the flow inlet. (The turbulence intensity is equal to $6.99 \%$ for the case here, where $\operatorname{Re}=3,000$ ). 


\subsection{Results and Discussion Using a LES Turbulence Model}

The flow in a six-element static mixer has been analyzed for a flow Reynolds number of 3,000. The fluid used in this study is incompressible with constant viscosity. The density and viscosity were set to $10^{4} \mathrm{~kg} / \mathrm{m}^{3}$ and $6.631 \times 10^{-4} \mathrm{~kg} / \mathrm{m} . \mathrm{s}$, respectively.

The time step was set to $2 \times 10^{-5}$ seconds. A scaled residual [123] less than $10^{-5}$ for all scalar equations was the convergence criterion at each time level. The number of iterations required to obtain a converged solution in each time step ranged from 4 to 8 . Using the base solution, obtained by the $k-\omega$ model, as initial conditions for the LES model, the solution was run for a physical time of one second, long enough to become independent of the initial condition and long enough to become statistically steady. Mass imbalance was monitored in one fifth of the computational cells, selected randomly. When the flow statistically converged, the maximum mass imbalance in a cell was found to be $9.25 \times 10^{-11} \mathrm{~kg} / \mathrm{s}$, and the averaged value of absolute values of mass imbalance in computational cells was found to be $7.58 \times 10^{-13} \mathrm{~kg} / \mathrm{s}$.

The pressure drop across the static mixer was computed. The mean value of the static pressure drop is $846.2491 \mathrm{~Pa}$. The minimum value of instantaneous pressure drop is 832.2518 $\mathrm{Pa}$ (at a physical time equal to $0.8055 \mathrm{~s}$ ), and its maximum value is $875.8536 \mathrm{~Pa}$ (at a physical time equal to $0.4727 \mathrm{~s}$ ). The standard deviation of the static pressure is 18.3643 Pa.

Figure 7.18 shows the mean static pressure contours depicted at two cross-sections: the upstream cross-section (located at a distance of L/2 upstream of the leading edge of 
the first element), and the downstream cross-section (located at a distance of $\mathrm{L} / 2$ downstream of the trailing edge of the last element), where $\mathrm{L}$ is the length of one mixing element. The mean static pressure contours at the end of each mixing element are shown in Fig. 7.19. The bulk pressure drop across the static mixer, predicted by less accurate (yet less computationally expensive) turbulence models, such as the $k-\varepsilon$ and the $k-\omega$ models, was shown to be reasonably accurate when compared with experimental data. The LES results can serve as a validation tool to further investigate the accuracy and details of the flow field predicted by those models.

The mean velocity and instantaneous velocity fields were computed. The crosssectional projection of the velocity vectors at the end of each mixing element are illustrated in Fig. 7.20. Different patterns at each cross-section can be seen. After the first mixing element, four velocity zones are observed, which are symmetric with respect to the flow center. Two of these zones contain large strong vortices; the two other zones contain weak vortices. After the second element, the velocity patterns in those zones are changed and four vortices with almost the same size are distinguishable. These zones combine together and create two velocity zones, as the flow goes through the mixer.

Table 7.10 presents averaged, maximum and minimum value of vorticity at the end of each mixing element, predicted by the LES model for $\operatorname{Re}=3,000$. Flow vorticity has a major role in the mixing of fluids through a static mixer. As can be seen, after the first mixing element four regions vorticity magnitudes are observed. These regions are symmetric with respect to the center of the cross-section. The vorticity magnitude and the local Reynolds number are low in a large part of the flow at this cross-section. Therefore, 
a highly mixed pattern of fluids cannot be expected after the first mixing element. These separated regions still exist after the second and the third mixing elements, however, higher vorticity magnitudes are observed. As the flow passes through the mixer more uniform patterns of the local Reynolds number and vorticity are generated. Although the maximum values of these parameters are reduced at the fourth, fifth and sixth mixing elements, compared to the first three elements, the averaged values of these parameters are increased correspondingly (the averaged vorticity magnitude at the first mixing element is $916.31 s^{-1}$ while it is $1,019.75 s^{-1}$ at the fourth mixing element).

Using the obtained mean velocity field, the trajectories of 501,740 particles injected into the top half of the mixer at flow inlet have been calculated. In Fig. 7.21, positions of the particles are plotted at the end of each mixing element. These plots illustrate the redistribution of the released particles via the combined effects of flow division and reversal, resulting in stretching and folding of the observed structures.

Comparing the distribution of particles at different cross-sections shows that, two fluid components are still separated after the first mixing element. Almost all of the particles are located on one side of the flow cross-section. Separated islands of flow are distinguishable after the flow passes the second mixing element. These islands are divided into several narrow regions when the flow passes the third and the forth mixing element. The fluid within these islands is spread over a larger area of the flow crosssection. After the sixth element, most of the flow cross section is filled with those smeared areas of the flow. These areas have a distinguishable curved shape. Also, the 
particles are distributed more uniformly in the mixer cross-section (the cross-sectional area of flow containing no particles is much smaller).

The instantaneous distributions of particles at the physical times equal to 0.7509 and 0.80546 seconds are shown in Fig. 7.22, at the end of the second, the fourth, and the sixth mixing elements. Due to the chaotic nature of the flow, the particle distributions are different from each other and also from those obtained using the mean velocity field; however, the patterns are similar at the same flow cross-section.

Figure 7.23 shows the distribution function for flow across a six-element helical static mixer inside a pipe, calculated for $\operatorname{Re}=3,000$. The non-dimensional time step, $d t^{*}$, is 0.01. The standard deviation of the distribution function is about 0.02 . Two modes are distinguishable, the first mode starting at about $t^{*}=0.5$ and the second mode starting at about $t^{*}=1.8$. The second mode is substantially weaker than the first mode. The first mode is associated with streamlines that occupy a large portion of the flow. For about 98\% of fluid particles, $t^{*}$ is between 0.5 and 1.5 ; and $t^{*}$ is between 0.7 and 1.3 for about $46 \%$ of fluid particles.

The PDU values after each mixing element are shown in Fig. 7.24. As can be seen, as the flow passes through the mixer the PDU value increases. The rate of increase of the PDU value is higher for the even numbered mixing elements rather than the odd numbered elements. A significant increase in PDU values can be seen at the fourth mixing element and more significantly at the second mixing element. This can be explained by considering the fact that mean vorticity magnitudes are larger at those elements. The averaged vorticity magnitude at the second mixing element is 1.15 times 
the averaged vorticity magnitude at the first element, and the averaged vorticity magnitude at the fourth mixing element is 1.04 times that at the third element.

The accuracy of the numerical solver affects the obtained PDU value. A more accurate turbulence model, as well as a more accurate discretization method, can capture a large amount of the flow physics of the problem and can thereby provide a more detailed solution. Therefore, even for fluid particles released very near each other, distinct paths are detectable. This feature is not achievable, using less accurate turbulence models, due to their inability to resolve small vortical structures. In fact it was observed, in practice, that using less accurate turbulence models, fluid particles released from neighboring points follow virtually indistinguishable pathlines.

The static pressure contours at the upstream and the downstream cross-sections of the flow predicted by the $k-\omega$ and the RSM models are shown in Fig. 7.25, using the same scale as the one employed in Fig. 7.18. Although, the contour patterns are alike, but pressure values are significantly different at the same locations in each of flow crosssections. Also, comparison between the cross-sectional component of velocity vectors at the end of mixing elements obtained by the $k-\omega$ and the RSM turbulence models and the cross-sectional component of velocity vectors at the end of mixing element s obtained by the LES model shows that the $k-\omega$ and the RSM models are not able to capture solution details such as small vortices, leading to less accurate prediction of mixer performance.

The PDU values obtained from the velocity fields predicted by the $k-\varepsilon$, the $k-\omega$, and the RSM turbulence models are significantly smaller than the PDU values obtained 
by the LES model, e.g., the PDU value at the second mixing element obtained by the LES model is about $21 \%$ higher than the one obtained by the RSM model, about $19 \%$ higher than the one obtained by the $k-\omega$ model, and it is about $33 \%$ higher than the one obtained by the $k-\varepsilon$ turbulence model. In fact when validated against the LES results, the least reliable mixing prediction is obtained from the $k-\varepsilon$ model; the PDU value at the fourth mixing element obtained by the LES model, is about $37 \%$ higher than the one obtained by the $k-\varepsilon$ model. 


\begin{tabular}{|c|c|c|c|}
\hline Number of cells & Model & Minimum y+ & Maximum y+ \\
\hline $2,296,158$ & Spalart-Allmaras & 0.11 & 3.01 \\
\hline $2,189,439$ & $k-\varepsilon$ & 0.11 & 3.03 \\
\hline $2,335,690$ & $k-\omega$ & 0.11 & 2.96 \\
\hline $2,377,705$ & RSM & 0.12 & 2.95 \\
\hline
\end{tabular}

Table 7.1: Mesh information for the turbulent flow cases $(\operatorname{Re}=3,000)$ 


\begin{tabular}{|c|c|c|c|}
\hline Iterations & $\begin{array}{c}\text { Pressure drop } \\
\text { ratio }\end{array}$ & $\begin{array}{c}\text { Averaged velocity } \\
\text { ratio at the 6th } \\
\text { element }\end{array}$ & $\begin{array}{c}\text { Averaged vorticity ratio } \\
\text { at the 6th element }\end{array}$ \\
\hline 3990 & 1.000424 & 1.000291 & 1.000440 \\
\hline 4190 & 0.999913 & 1.000028 & 1.000045 \\
\hline 4390 & 1.000018 & 1.000103 & 1.000214 \\
\hline 4590 & 0.999998 & 0.999998 & 0.999968 \\
\hline 4790 & 0.999999 & 1.000002 & 1.000003 \\
\hline 4820 & 1.000000 & 1.000000 & 1.000000 \\
\hline
\end{tabular}

Table 7.2: Convergence of pressure drop across the mixer 


\begin{tabular}{|c|c|c|c|}
\hline Model & Iterations & CPU time per iteration & Memory \\
\hline Spalart-Allmaras & 5650 & 0.60 & 0.98 \\
\hline$k-\varepsilon$ & 3650 & 0.70 & 0.99 \\
\hline$k-\omega$ & 4820 & 1.00 & 1.00 \\
\hline RSM & 6000 & 1.19 & 1.11 \\
\hline
\end{tabular}

Table 7.3: CPU time and memory used by different turbulence models $(\operatorname{Re}=3,000)$ 


\begin{tabular}{|c|c|c|c|c|c|c|}
\cline { 2 - 7 } \multirow{2}{*}{ Model } & \multicolumn{3}{|c|}{ Mean vorticity } & \multicolumn{3}{c|}{ Maximum vorticity } \\
& \multicolumn{2}{|c|}{$1 / s$} & & \multicolumn{3}{c|}{$1 / s$} \\
\cline { 2 - 7 } & 2nd & 4th & 6th & 2nd & 4th & 6th \\
\hline Spalart-Allmaras & 2679.29 & 2775.27 & 243.41 & 20751.85 & 19541.44 & 6782.05 \\
\hline$k-\varepsilon$ & 2569.67 & 2579.63 & 187.68 & 19814.05 & 19815.00 & 4945.28 \\
\hline$k-\omega$ & 2700.04 & 2791.02 & 249.25 & 20481.51 & 19247.37 & 5593.88 \\
\hline RSM & 2731.08 & 2631.31 & 166.18 & 19450.66 & 19595.32 & 5097.55 \\
\hline
\end{tabular}

Table 7.4: Vorticity magnitude at $2 \mathrm{nd}, 4$ th, and 6 th mixing element $(\operatorname{Re}=3,000)$ 


\begin{tabular}{|c|c|}
\hline Model & $\begin{array}{c}\text { Pressure drop } \\
(\mathrm{Pa})\end{array}$ \\
\hline Spalart-Allmaras & 1161.43 \\
\hline$k-\varepsilon$ & 1129.78 \\
\hline$k-\omega$ & 1167.41 \\
\hline RSM & 1185.77 \\
\hline
\end{tabular}

Table 7.5: Pressure drop predicted by different turbulence models $(\operatorname{Re}=3,000)$ 


\begin{tabular}{|c|c|}
\hline Model & $\begin{array}{c}\text { G-value } \\
1 / s\end{array}$ \\
\hline Spalart-Allmaras & 1975.04 \\
\hline$k-\varepsilon$ & 1947.94 \\
\hline$k-\omega$ & 1980.12 \\
\hline RSM & 1995.63 \\
\hline
\end{tabular}

Table 7.6: G-value for a six-element static mixer $(\operatorname{Re}=3,000)$ 


\begin{tabular}{|c|c|c|c|}
\cline { 2 - 4 } Model & \multicolumn{3}{|c|}{ Structure radius } \\
\cline { 2 - 4 } & 2nd & 4th & 6th \\
\hline Spalart-Allmaras & 0.0408 & 0.0155 & 0.0155 \\
\hline$k-\varepsilon$ & 0.0408 & 0.0163 & 0.0102 \\
\hline$k-\omega$ & 0.0347 & 0.0155 & 0.0159 \\
\hline RSM & 0.0347 & 0.0151 & 0.0131 \\
\hline
\end{tabular}

Table 7.7: Structure radius at 2nd, 4th, and 6th mixing element $(\operatorname{Re}=3,000)$ 


\begin{tabular}{|c|c|}
\hline Model & Standard Deviation \\
\hline Spalart-Allmaras & $1.8663 \mathrm{E}-002$ \\
\hline$k-\varepsilon$ & $1.2930 \mathrm{E}-002$ \\
\hline$k-\omega$ & $1.8726 \mathrm{E}-002$ \\
\hline RSM & $1.9107 \mathrm{E}-002$ \\
\hline
\end{tabular}

Table 7.8: Standard deviation of distribution function 


\begin{tabular}{|c|c|}
\hline Aspect ratio & $1.009279 \sim 1.009901$ \\
\hline Diagonal ratio & $0.999989 \sim 1.000001$ \\
\hline Edge ratio & $1.008078 \sim 1.009697$ \\
\hline Mid-angle skewness & $<0.000001$ \\
\hline Equal-size skewness & $<0.000001$ \\
\hline
\end{tabular}

Table 7.9: Mesh quality test results for the LES case 


\begin{tabular}{|c|c|c|c|}
\hline $\begin{array}{c}\text { Mixing } \\
\text { Element }\end{array}$ & $\begin{array}{c}\text { Mean vorticity } \\
1 / \mathrm{s}\end{array}$ & $\begin{array}{c}\text { Maximum vorticity } \\
1 / \mathrm{s}\end{array}$ & $\begin{array}{c}\text { Minimum vorticity } \\
1 / \mathrm{s}\end{array}$ \\
\hline 1st & 916.3 & 3224.9 & 93.5 \\
\hline 2nd & 1057.8 & 4320.0 & 245.5 \\
\hline 3rd & 979.4 & 3006.8 & 304.8 \\
\hline 4th & 1019.7 & 3462.4 & 84.5 \\
\hline 5th & 960.2 & 2429.8 & 271.3 \\
\hline 6th & 703.6 & 1710.9 & 151.7 \\
\hline
\end{tabular}

Table 7.10: Maximum, minimum, and averaged vorticity magnitudes $(\operatorname{Re}=3,000)$ 

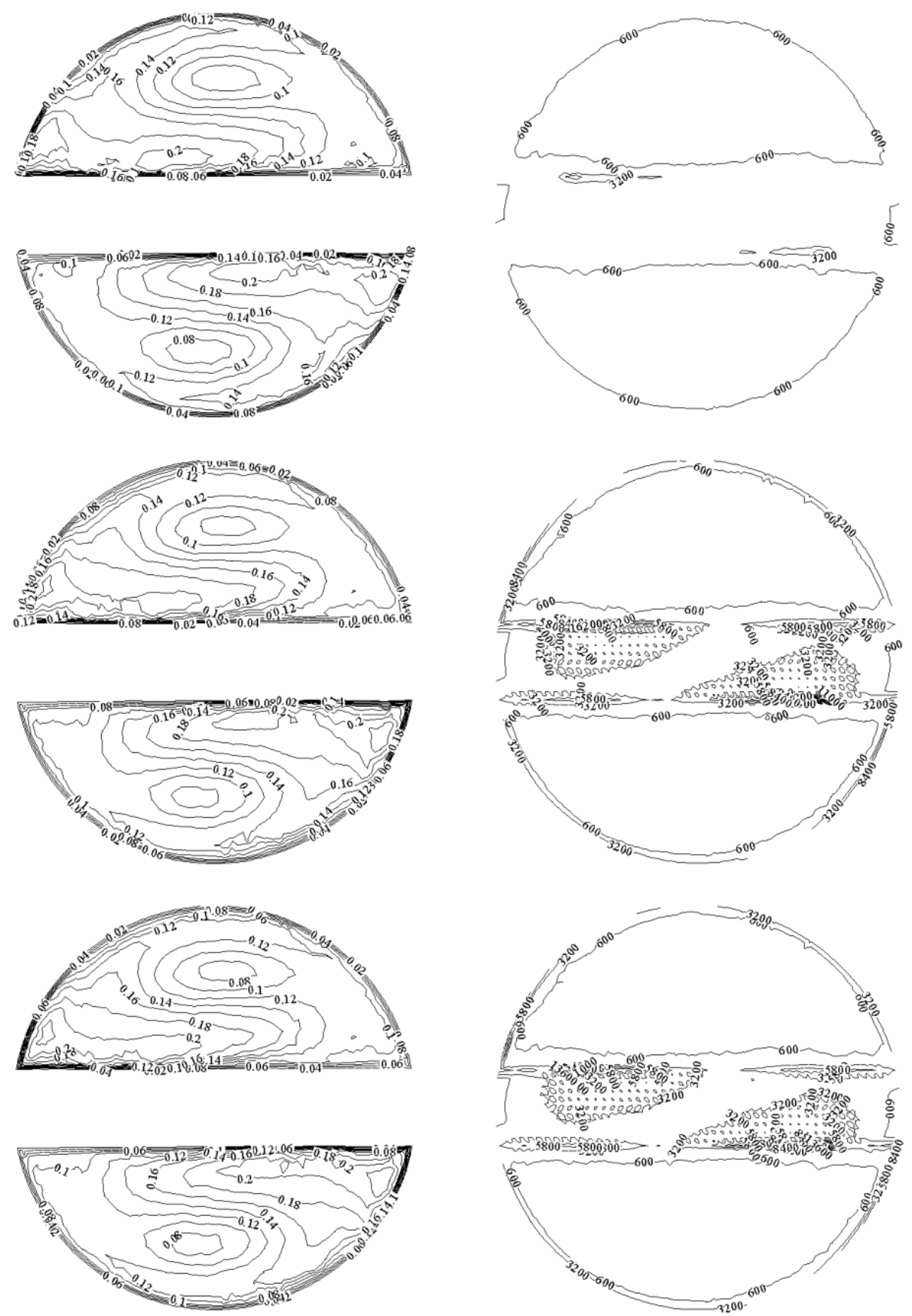

Figure 7.1: Velocity contours $(\mathrm{m} / \mathrm{s})$ and the vorticity contours $(1 / \mathrm{s})$ for $\operatorname{Re}=\mathbf{5 , 0 0 0}$ (using the $k-\omega$ model) 

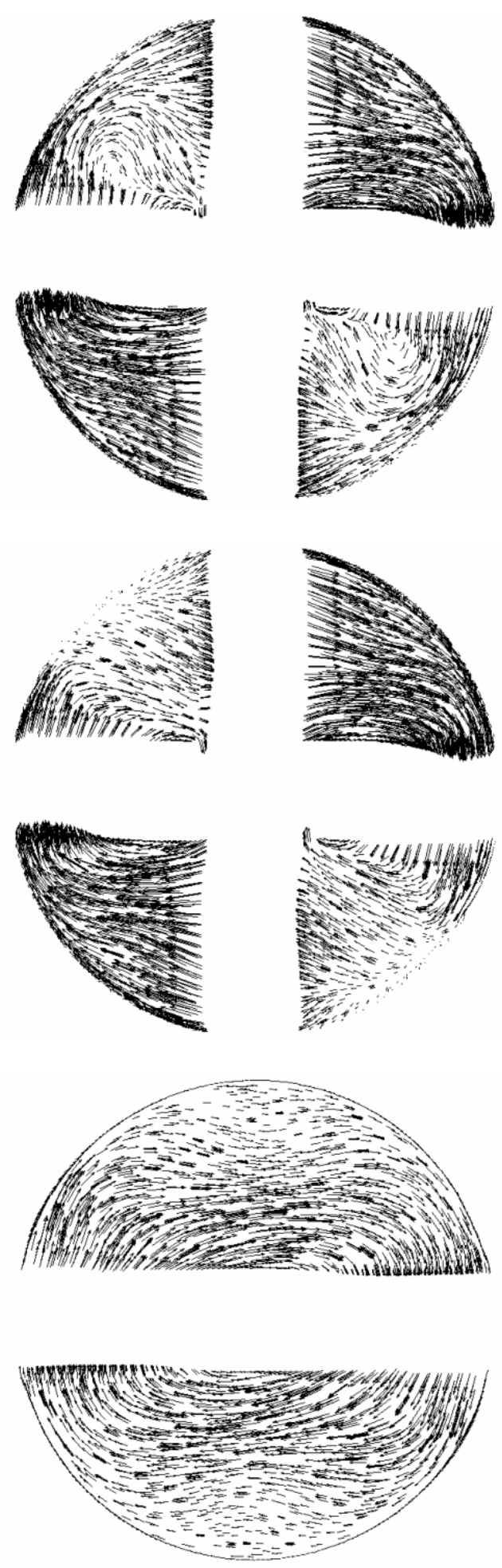

Figure 7.2: Velocity field, from top to bottom: at 2nd, 4th, and 6th mixing element, predicted by Spalart-Allmaras model 

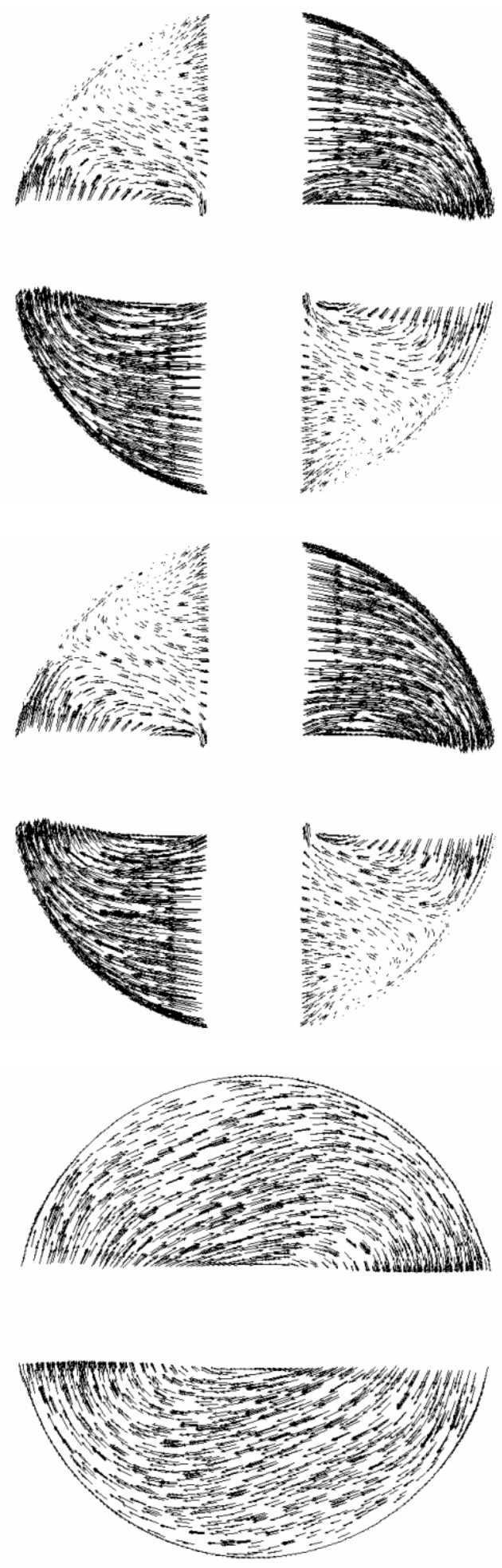

Figure 7.3: Velocity field, from top to bottom: at 2nd, 4th, and 6th mixing element, predicted by standard $k-\varepsilon$ model 

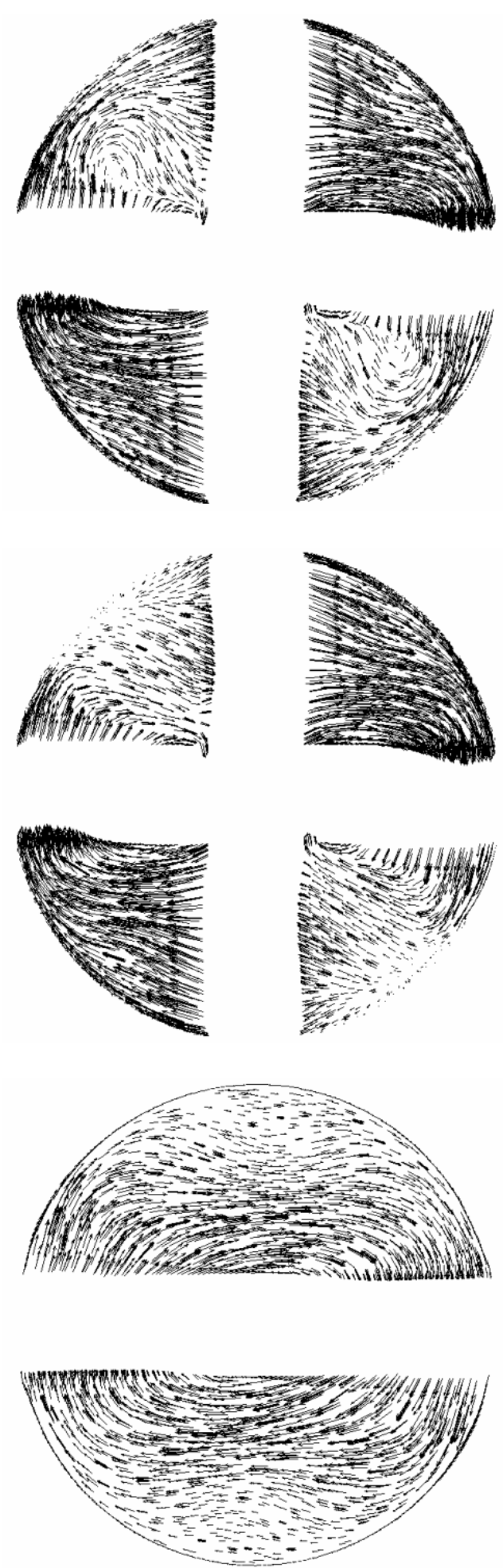

Figure 7.4: Velocity field, from top to bottom: at 2nd, 4th, and 6th mixing element, predicted by SST $k-\omega$ model 

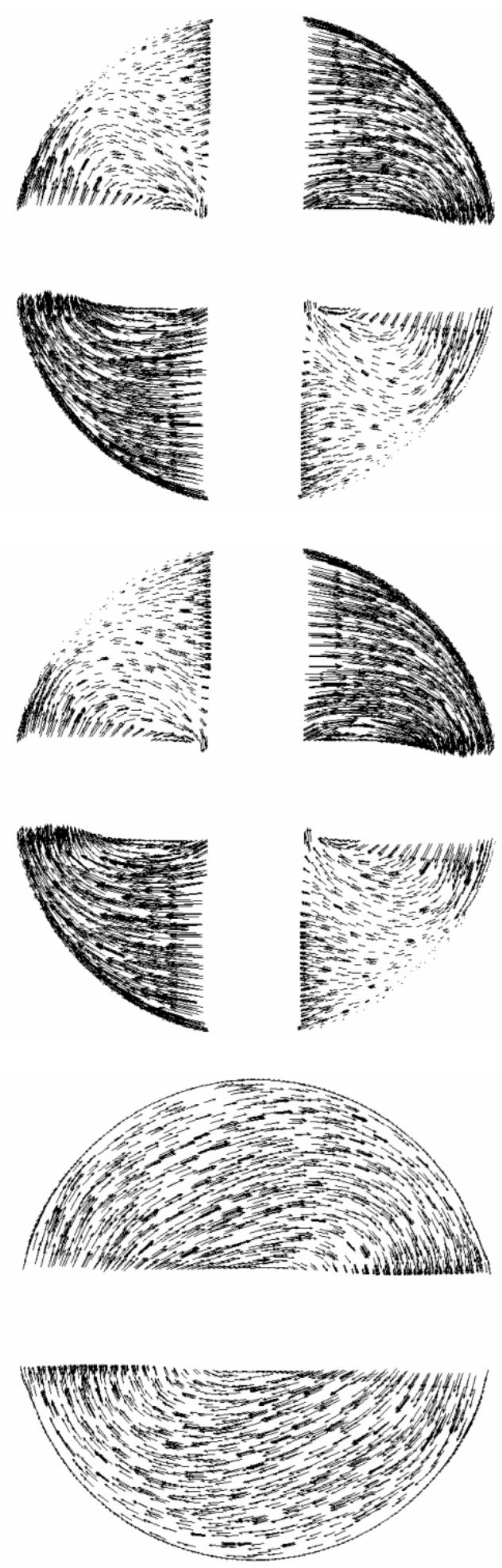

Figure 7.5: Velocity field, from top to bottom: at 2nd, 4th, and 6th mixing element, predicted by RSM model 


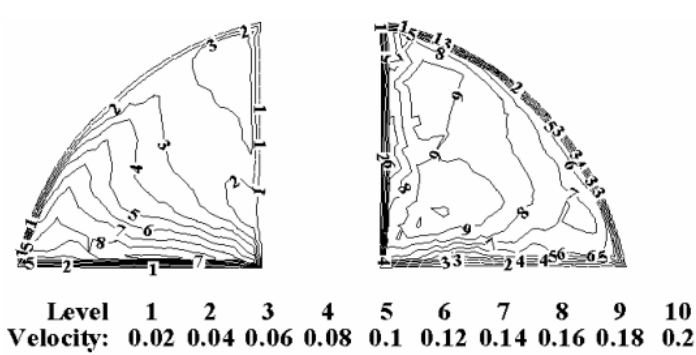

$\begin{array}{lllllllllll}\text { Velocity: } & 0.02 & 0.04 & 0.06 & 0.08 & 5 & 6 & 7 & 8 & 9 & 10\end{array}$
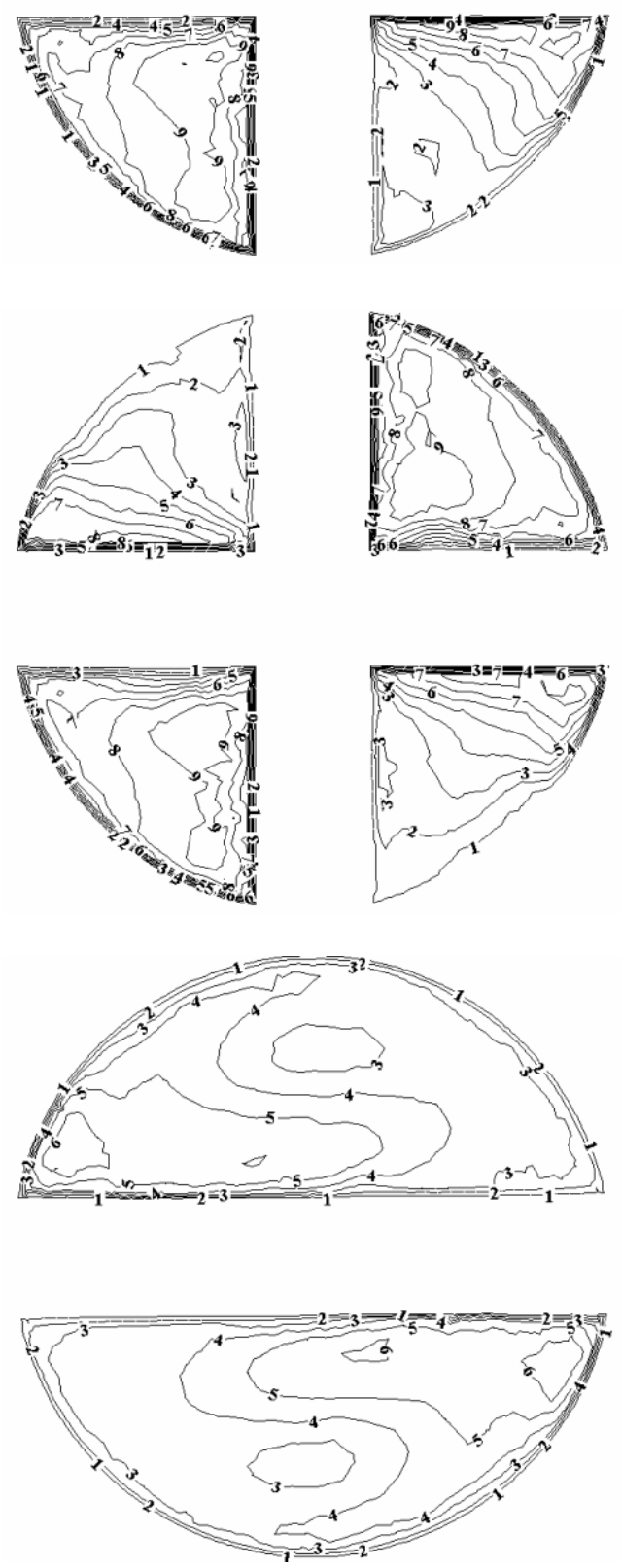

Figure 7.6: Velocity contours (m/s), from top to bottom: at 2nd, 4th, and 6th element, predicted by Spalart-Allmaras model 

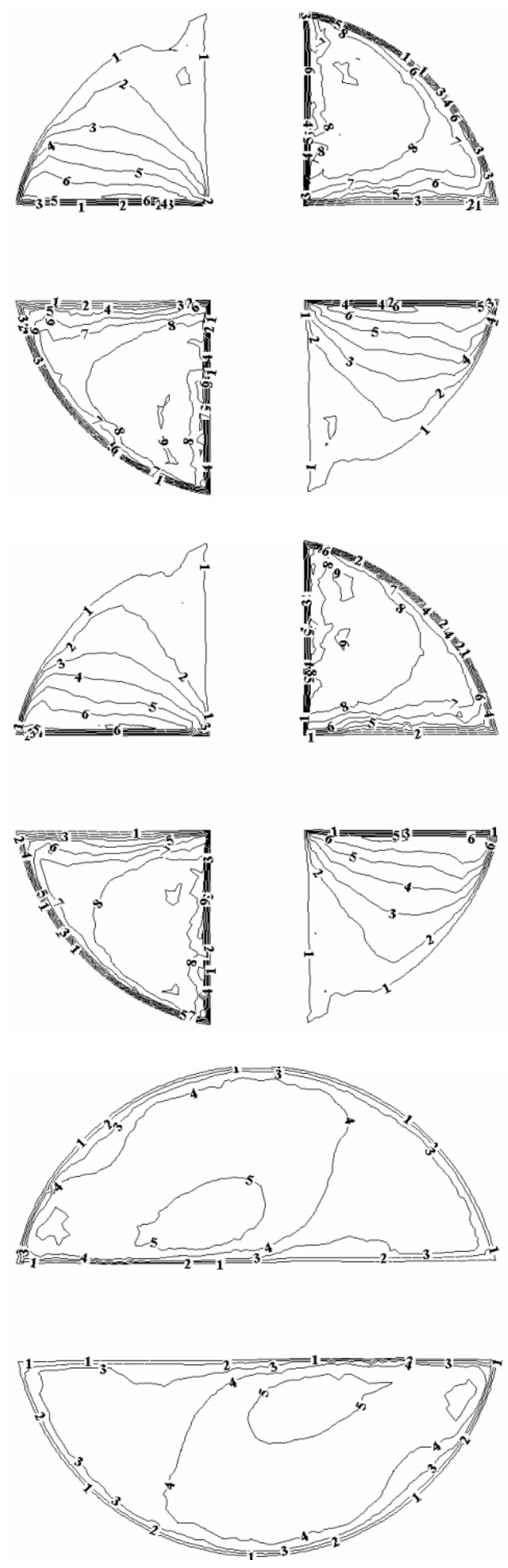

Figure 7.7: Velocity contours (m/s), from top to bottom: at 2nd, 4th, and 6th element, predicted by standard $k-\varepsilon$ model 

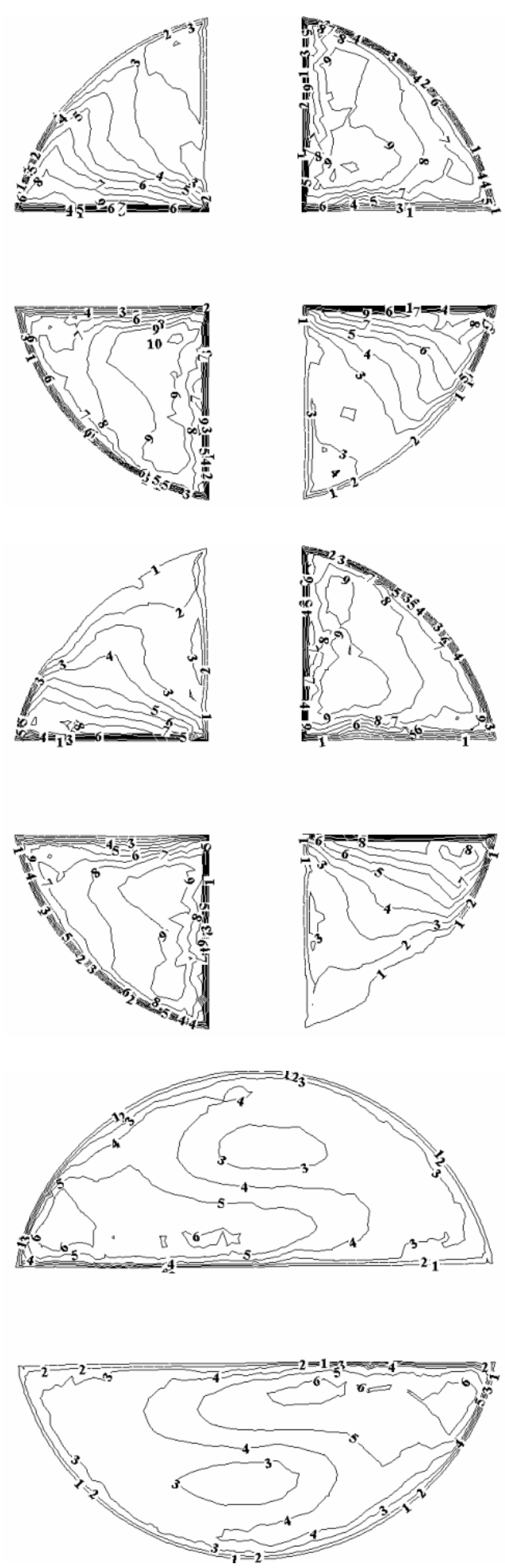

Figure 7.8: Velocity contours ( $(\mathrm{m} / \mathrm{s})$, from top to bottom: at $2 \mathrm{nd}, 4 \mathrm{th}$, and 6th element, predicted by standard SST $k-\omega$ model 

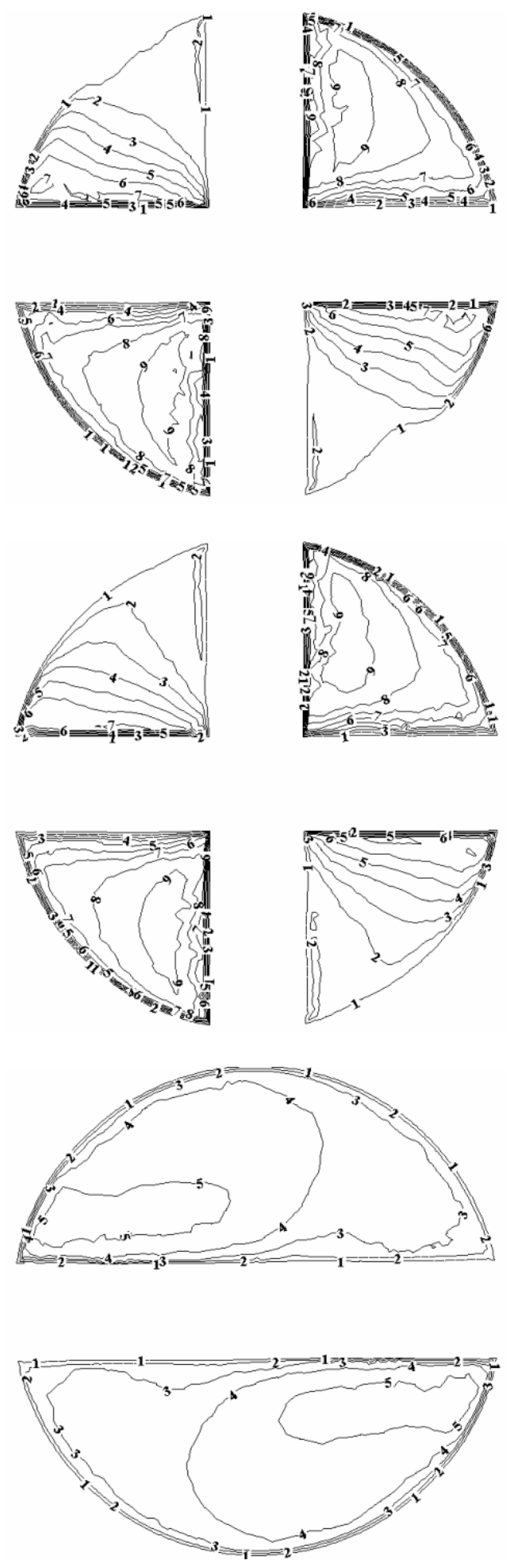

Figure 7.9: Velocity contours (m/s), from top to bottom: at 2 nd, 4 th, and 6th element, predicted by standard RSM model 

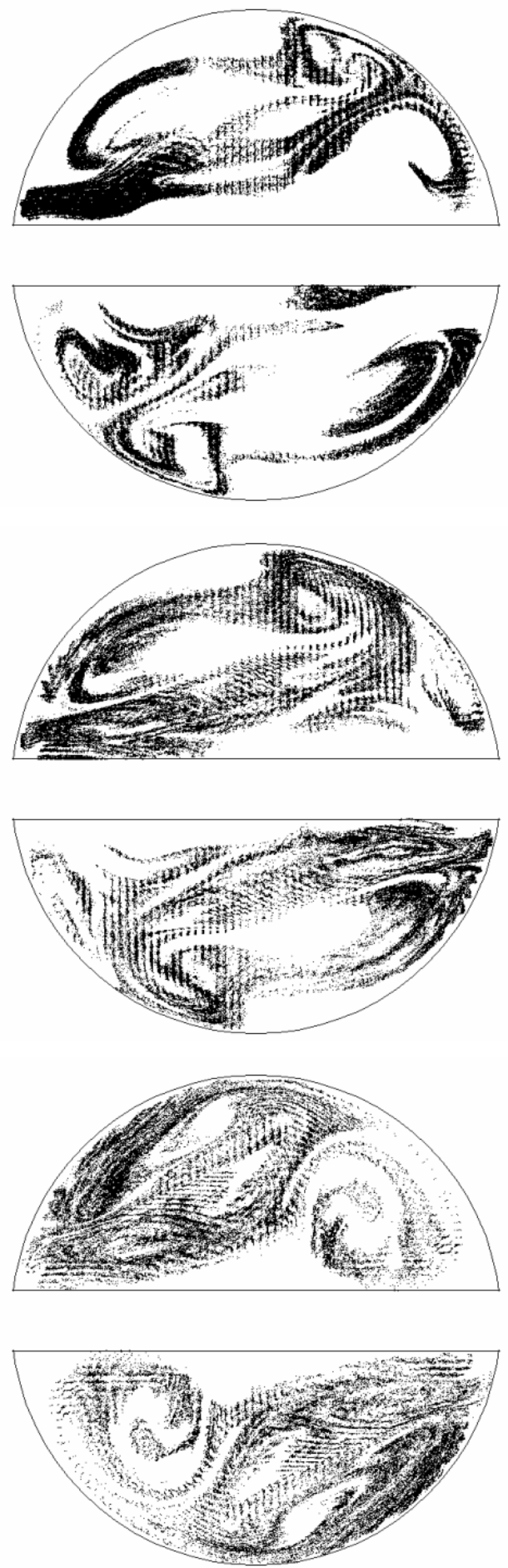

Figure 7.10: Particles locations at 2nd, 4th, and 6th element Predicted by Spalart-Allmaras model 

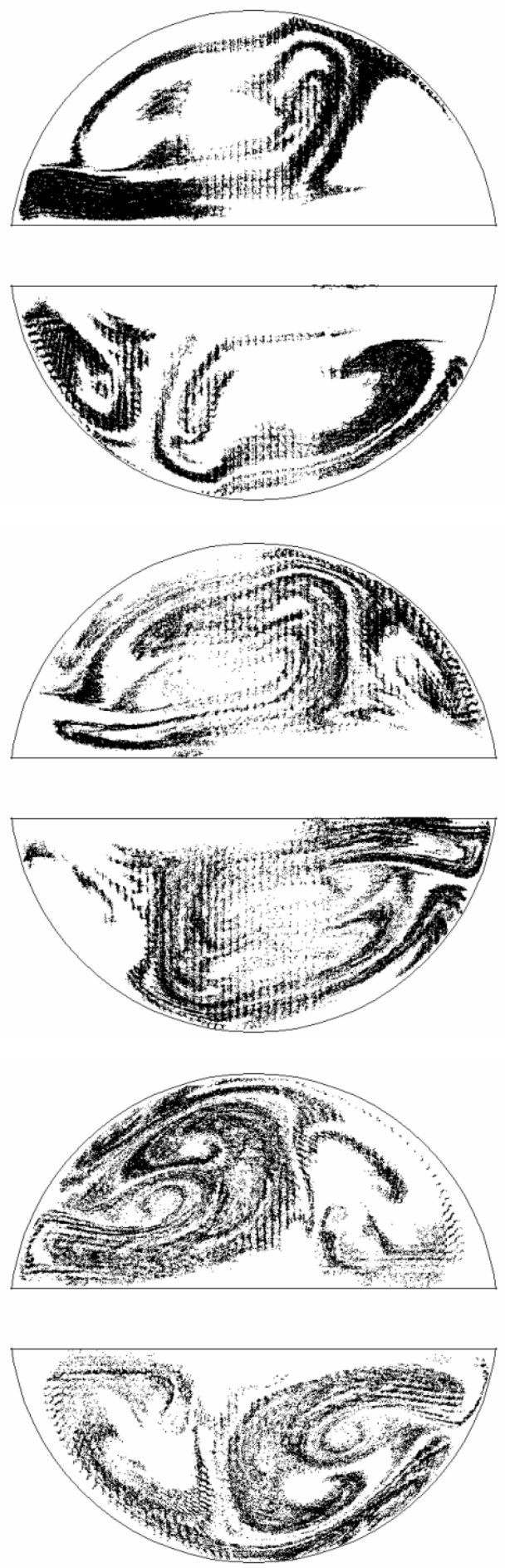

Figure 7.11: Particles locations at 2nd, 4th, and 6th element

Predicted by standard $k-\varepsilon$ model 

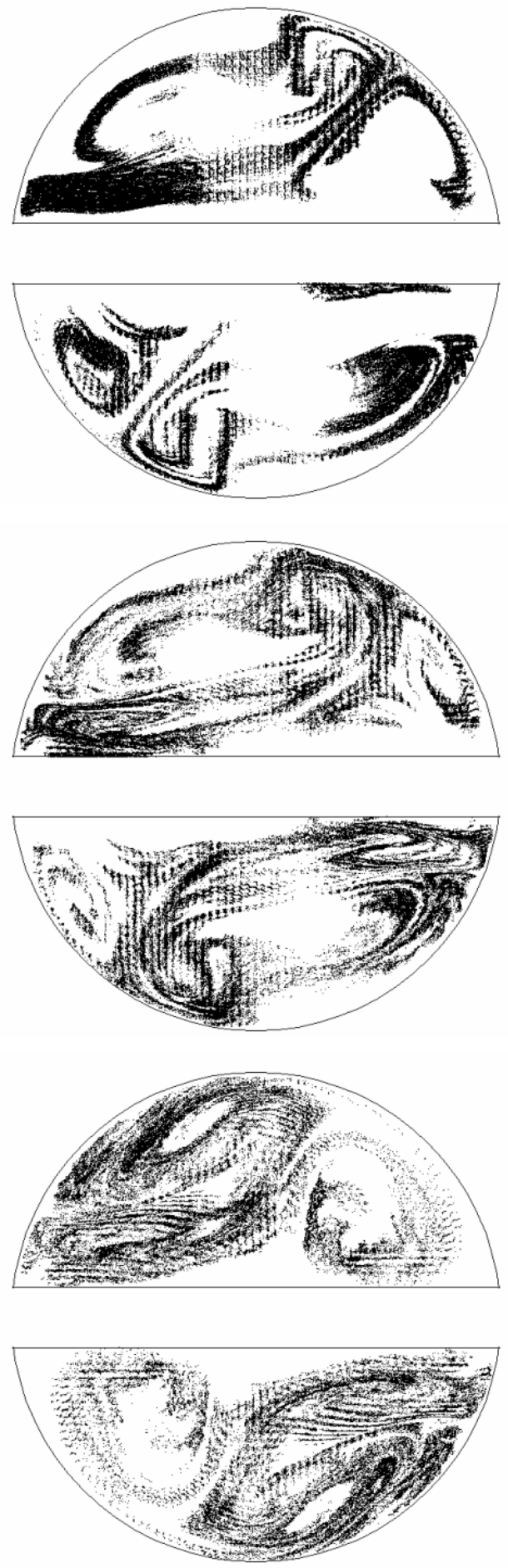

Figure 7.12: Particles locations at 2nd, 4th, and 6th element

Predicted by SST $k-\omega$ model 

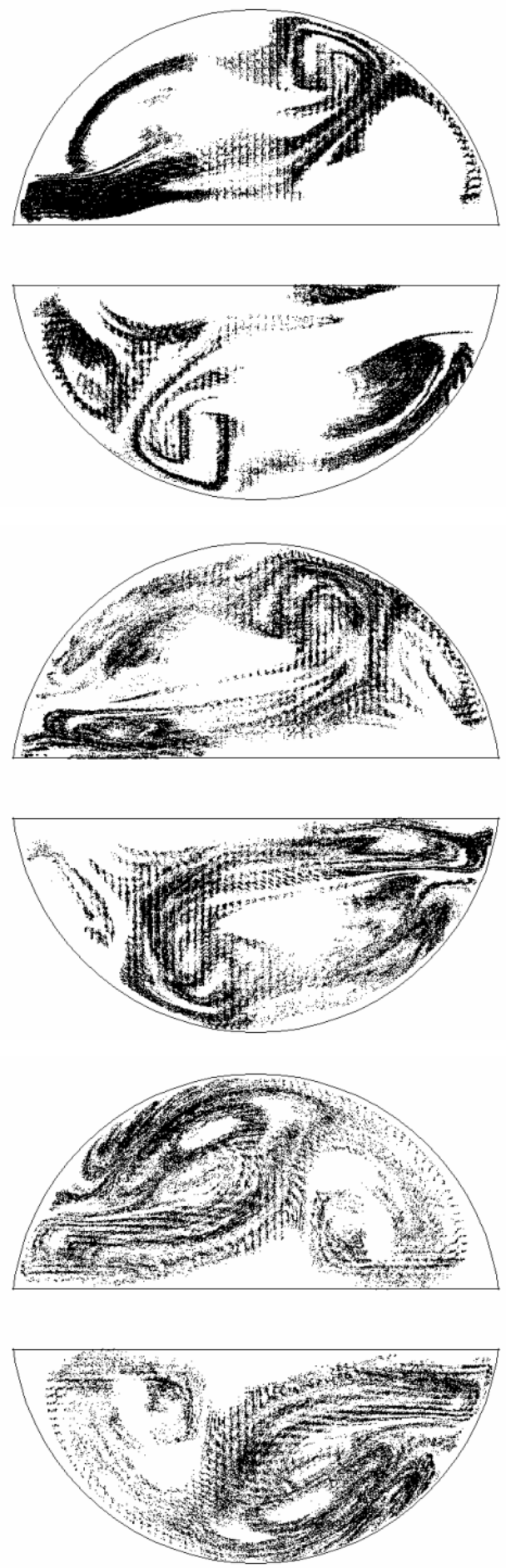

Figure 7.13: Particles locations at 2nd, 4th, and 6th element Predicted by RSM model 


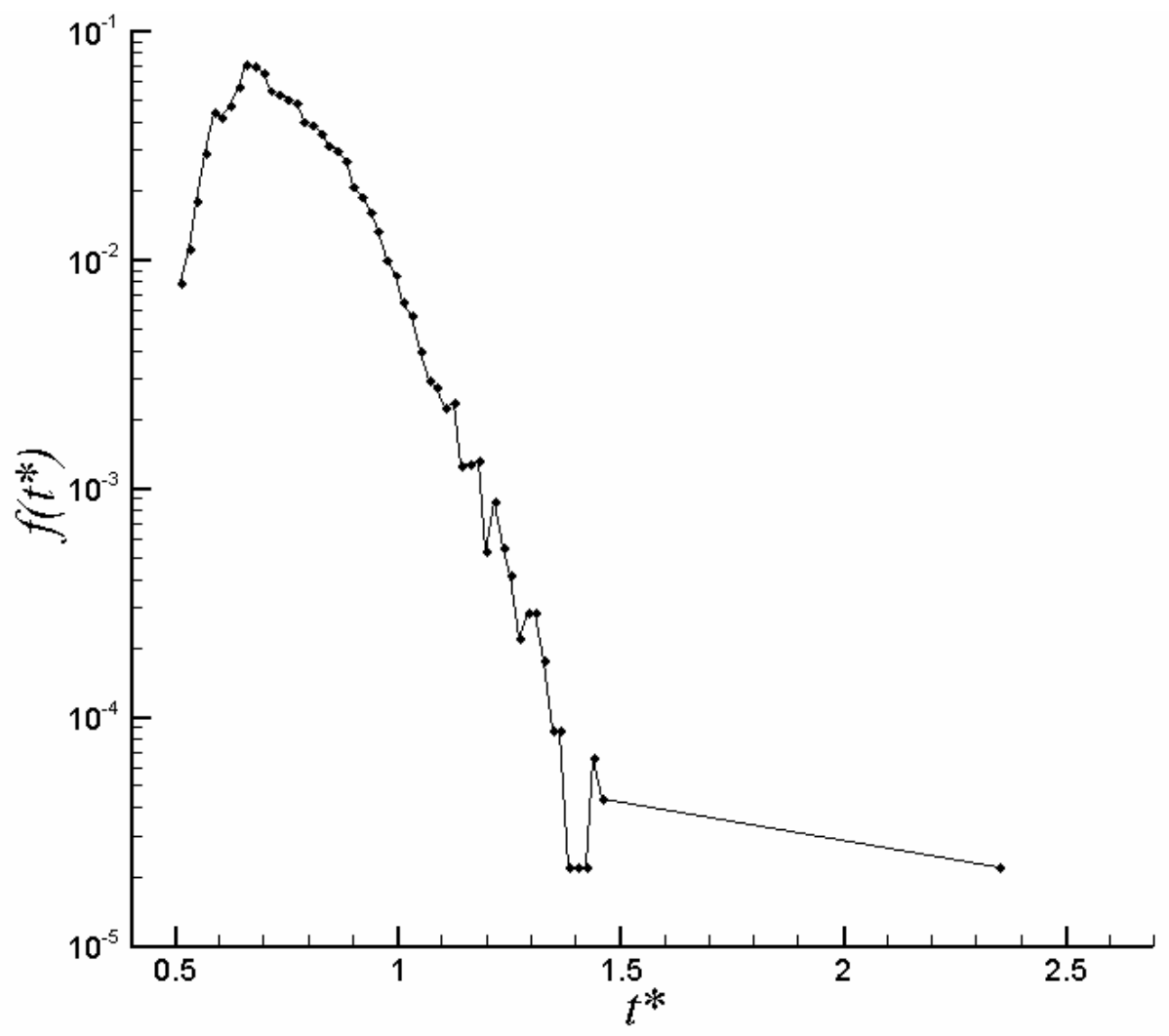

Figure 7.14: Distribution function for flow in the six-element static mixer, predicted by Spalart-Allmaras model 


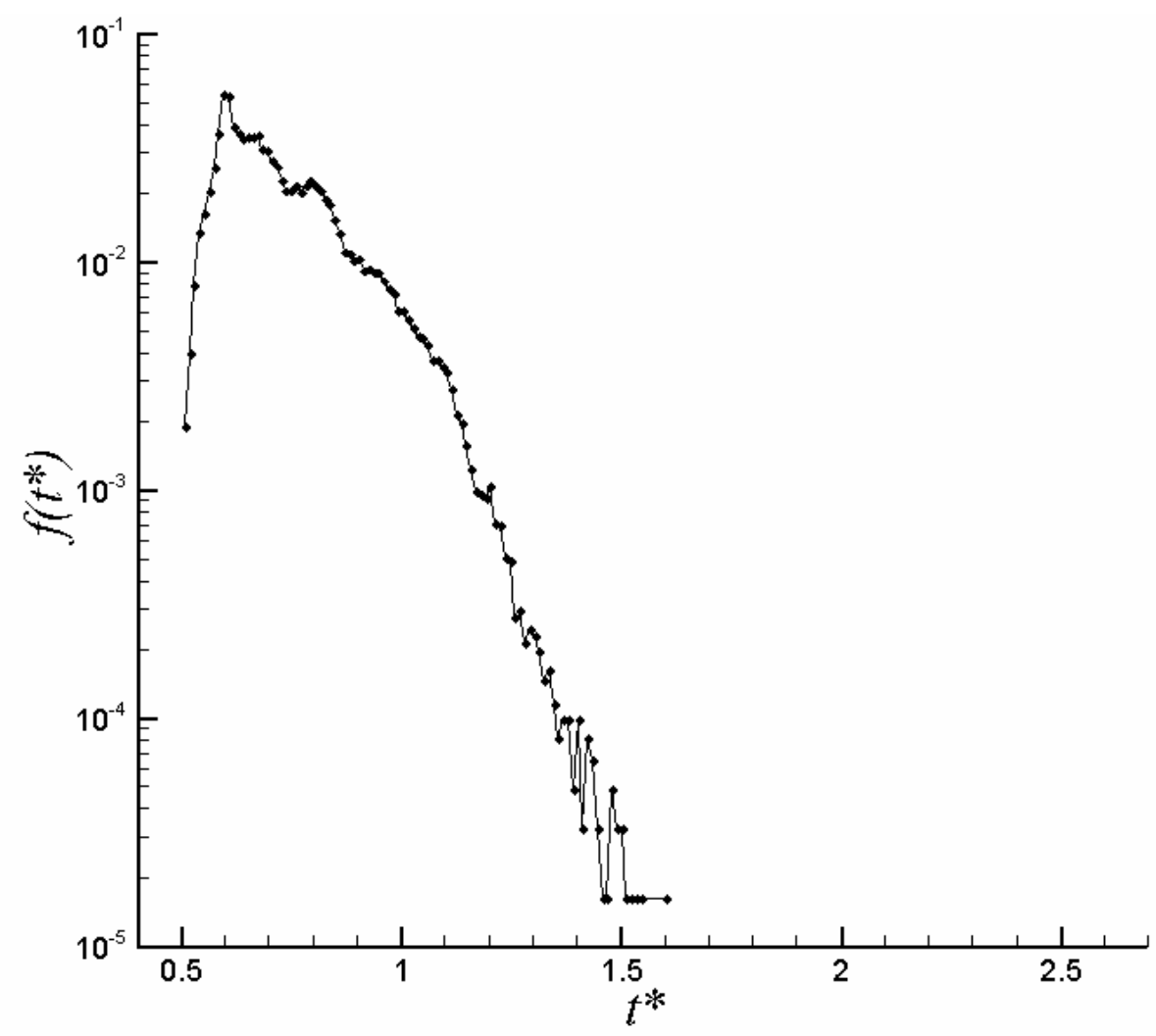

Figure 7.15: Distribution function for flow in the six-element static mixer, predicted by standard $k-\varepsilon$ model 


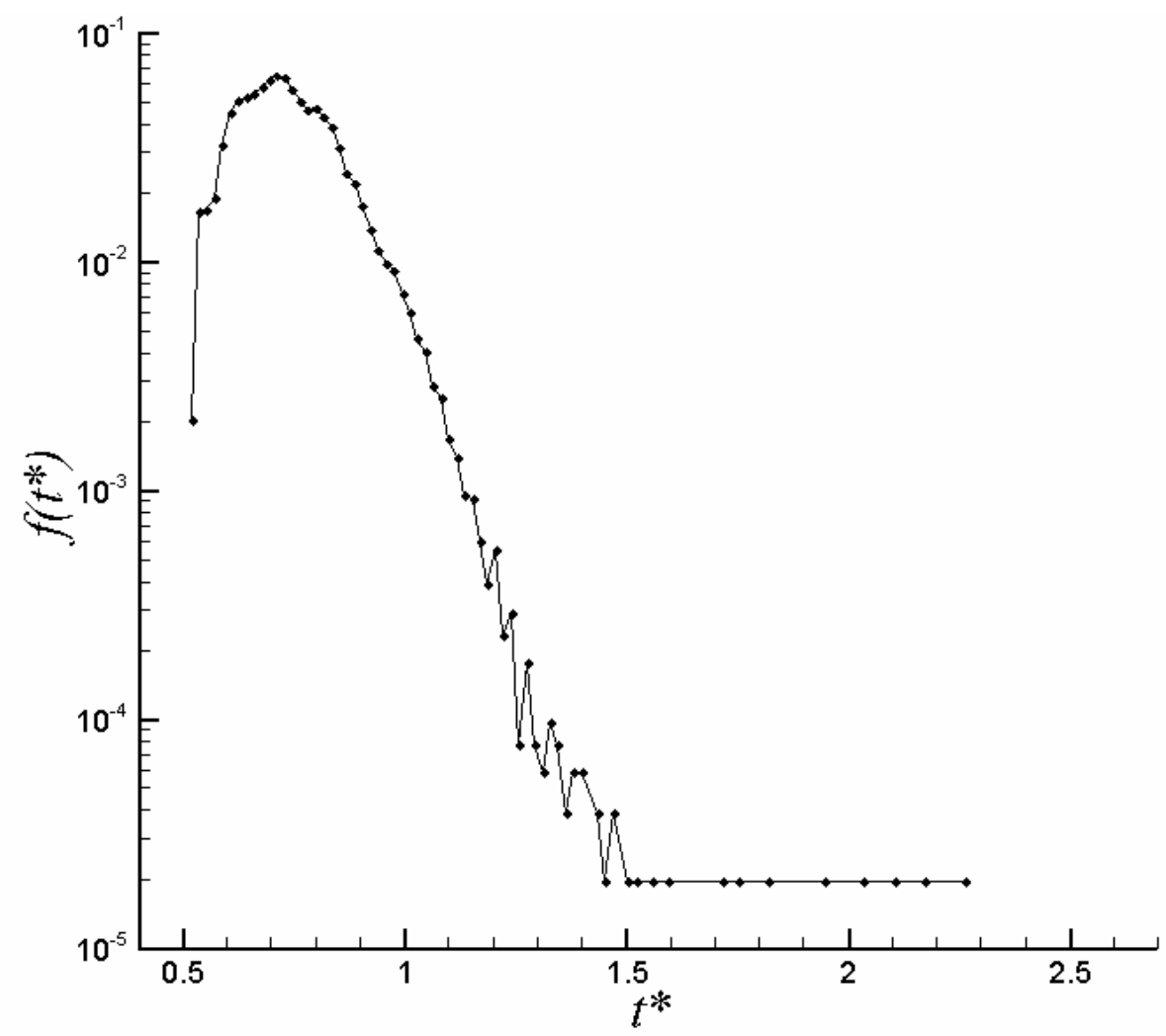

Figure 7.16: Distribution function for flow in the six-element static mixer, predicted by SST $k-\omega$ model 


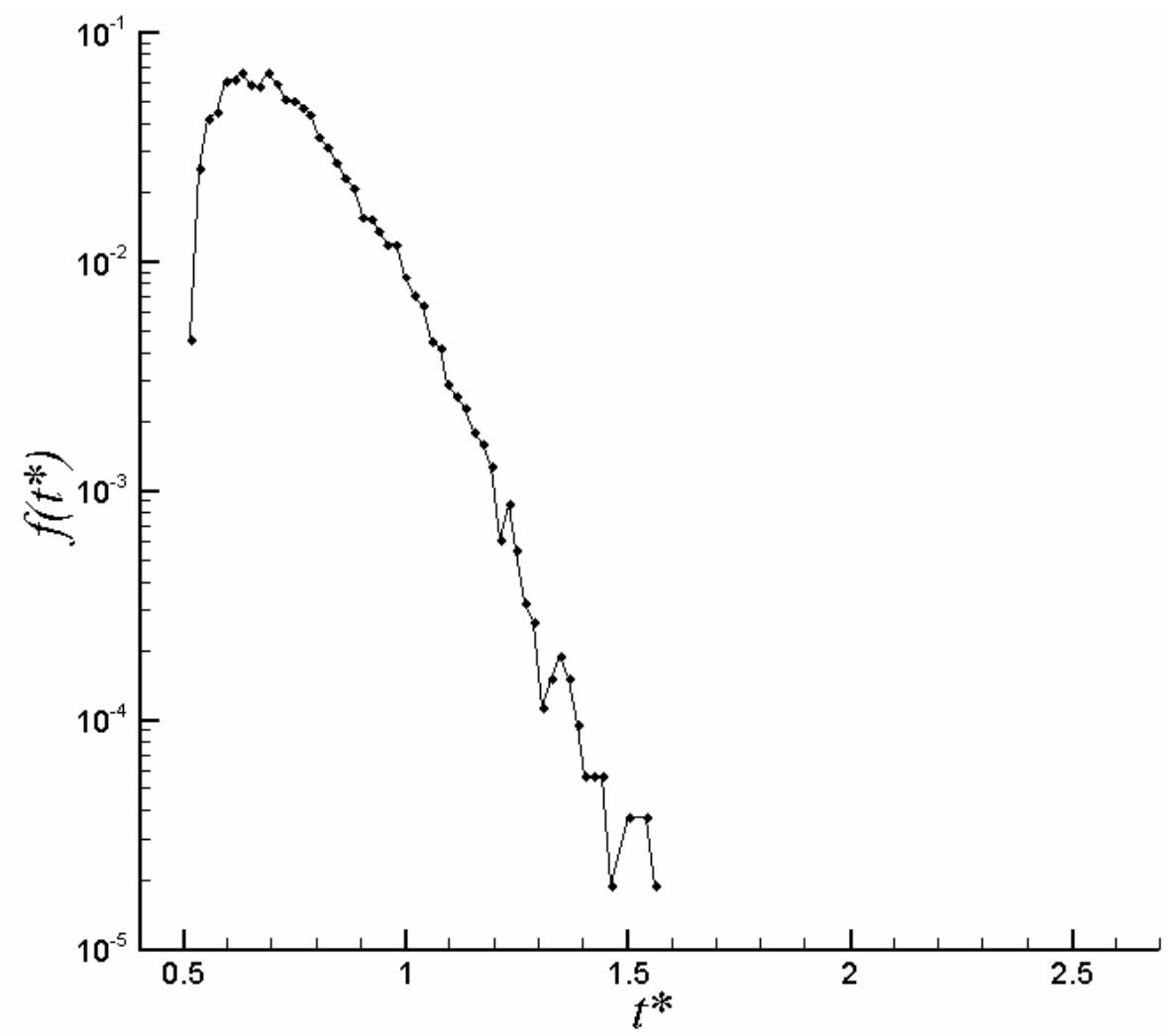

Figure 7.17: Distribution function for flow in the six-element static mixer, predicted by RSM model 
274

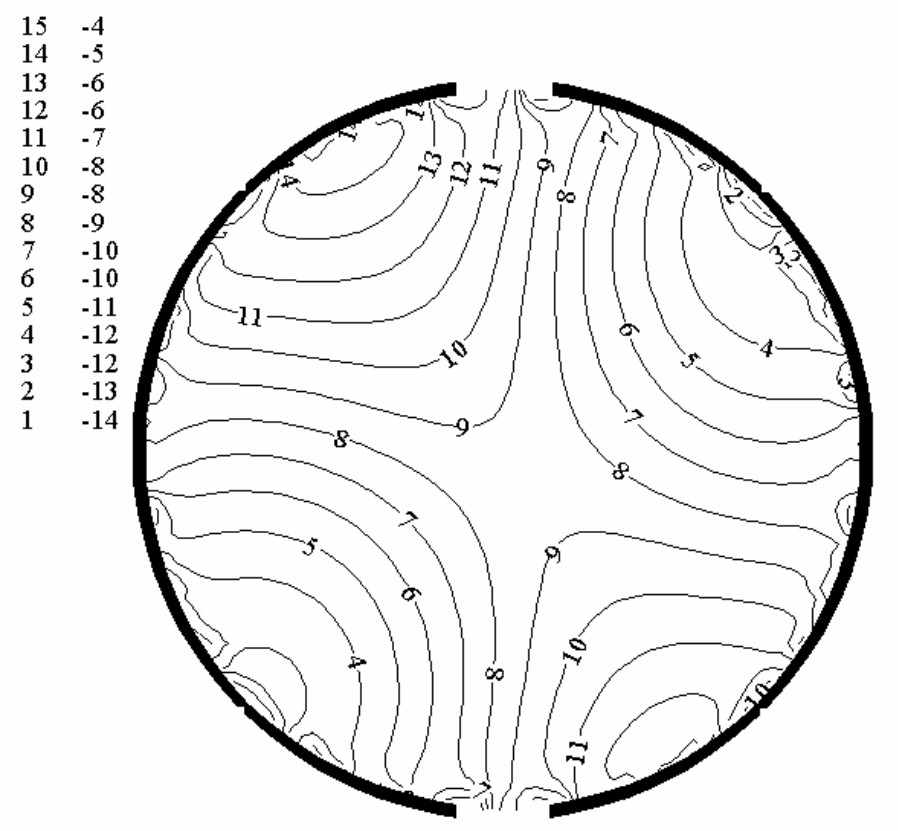

Pressure contours at up-stream cross-section

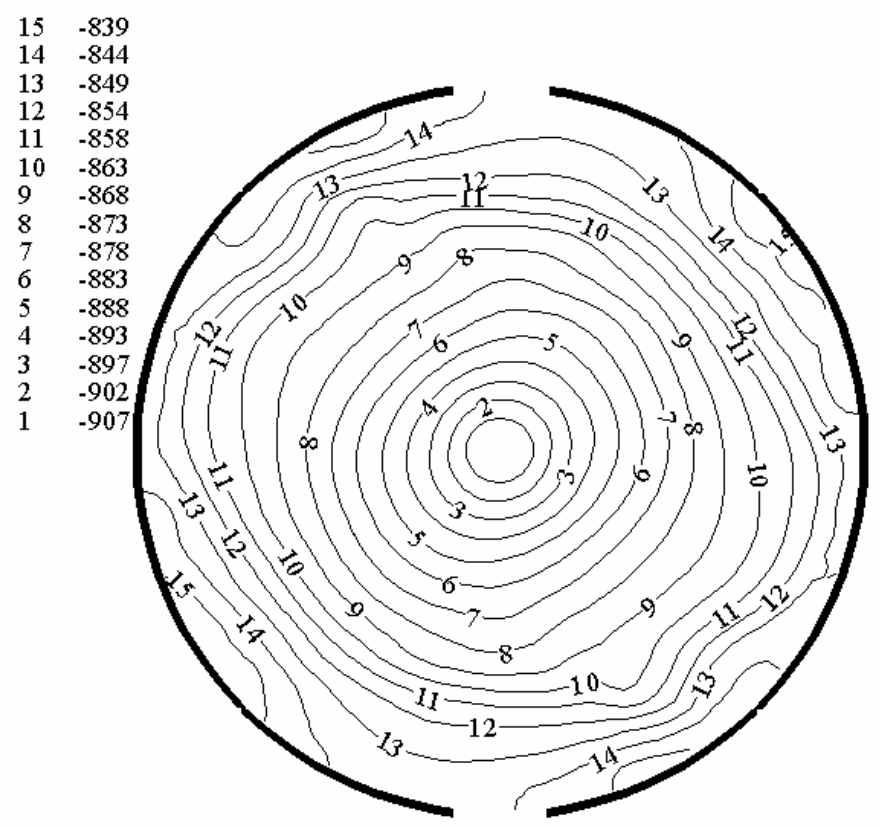

Pressure contours at downstream cross-section

Figure 7.18: Static pressure at the upstream and the down stream locations of a sixelement static mixer $(\mathbf{P a})$ 

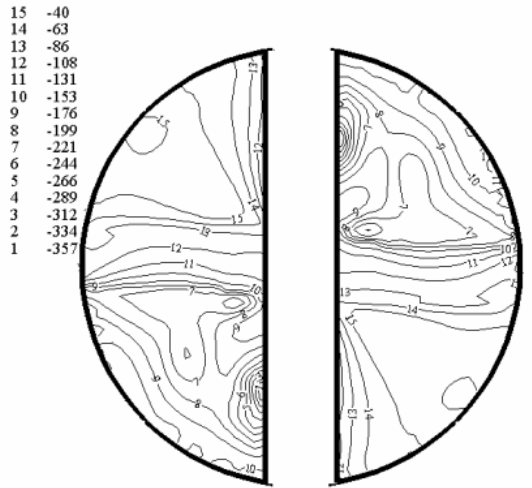

Pressure contours at the 1st mixing element

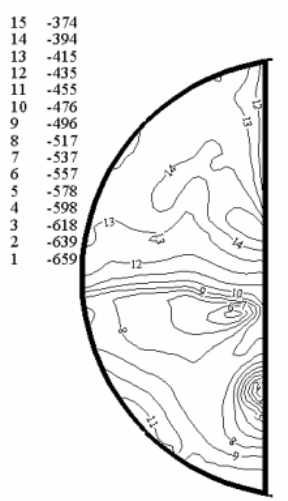

Pressure contours at the 3rd mixing element

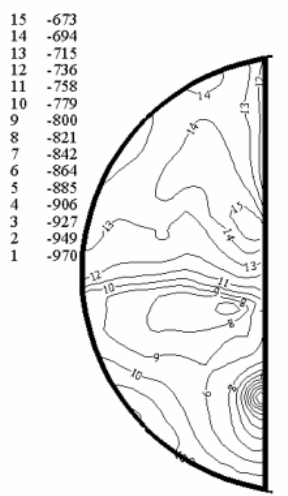

Pressure contours at the 5th mixing element

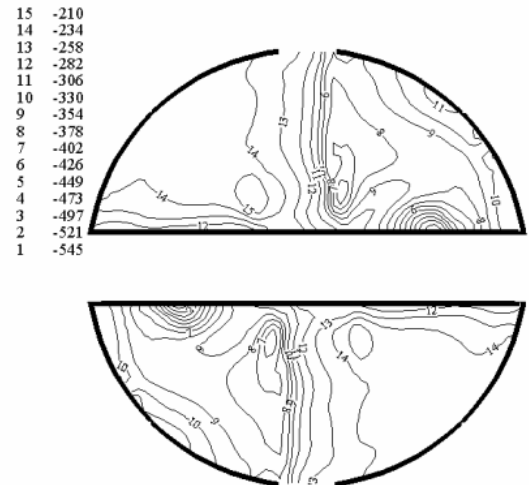

Pressure contours at the 2nd mixing element
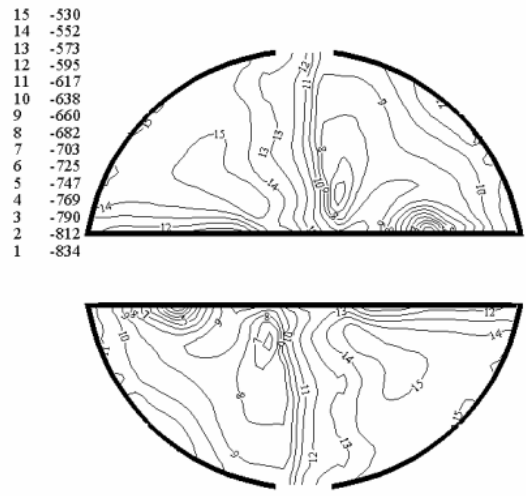

Pressure contours at the 4 th mixing element
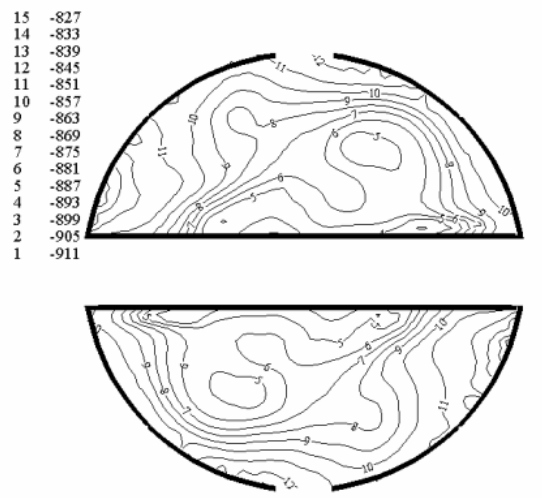

Pressure contours at the 6th mixing element

Figure 7.19: Static pressure at end of the 1st, 2nd, 3rd, 4th, 5th, and 6th mixing elements (Pa) 

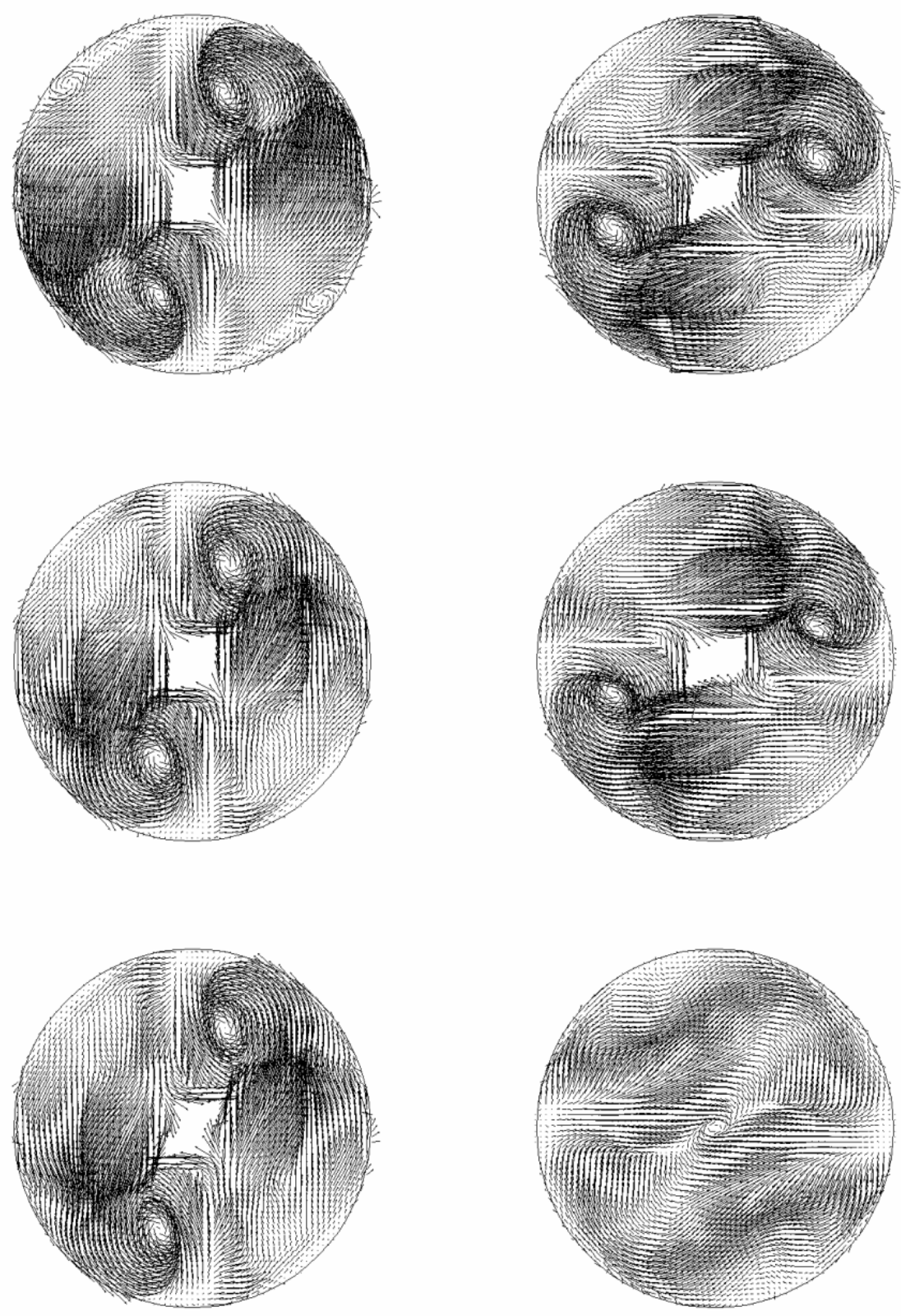

Figure 7.20: Cross-sectional velocity vectors at each mixing element cross section (from left to right, top to bottom at the end of the 1st, 2nd, 3rd, 4th, 5th, and 6th elements) 

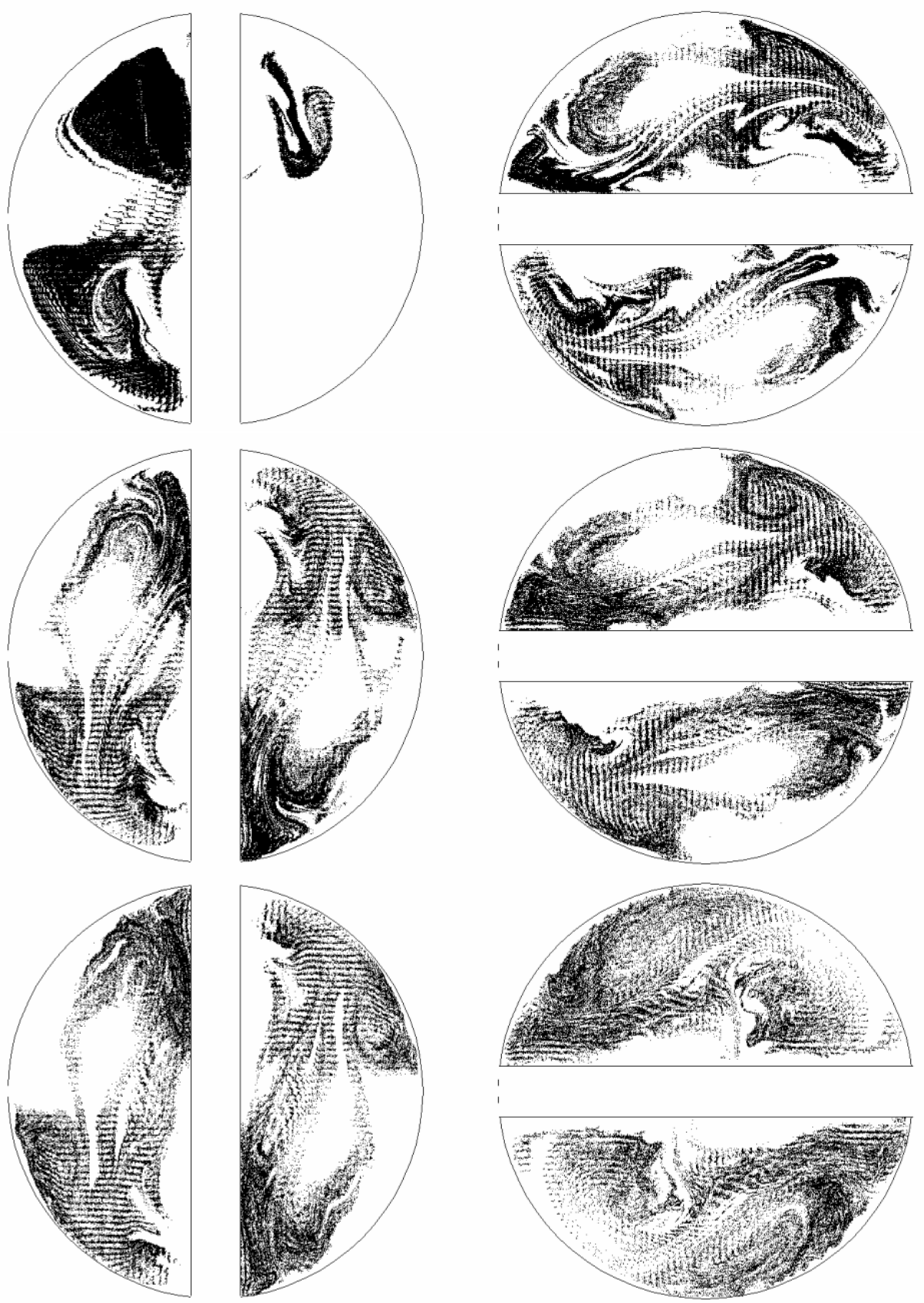

Figure 7.21: Particle locations the end of each mixing element (from left to right, top to bottom at the end of 1st, 2nd, 3rd, 4th, 5th, and 6th elements) 

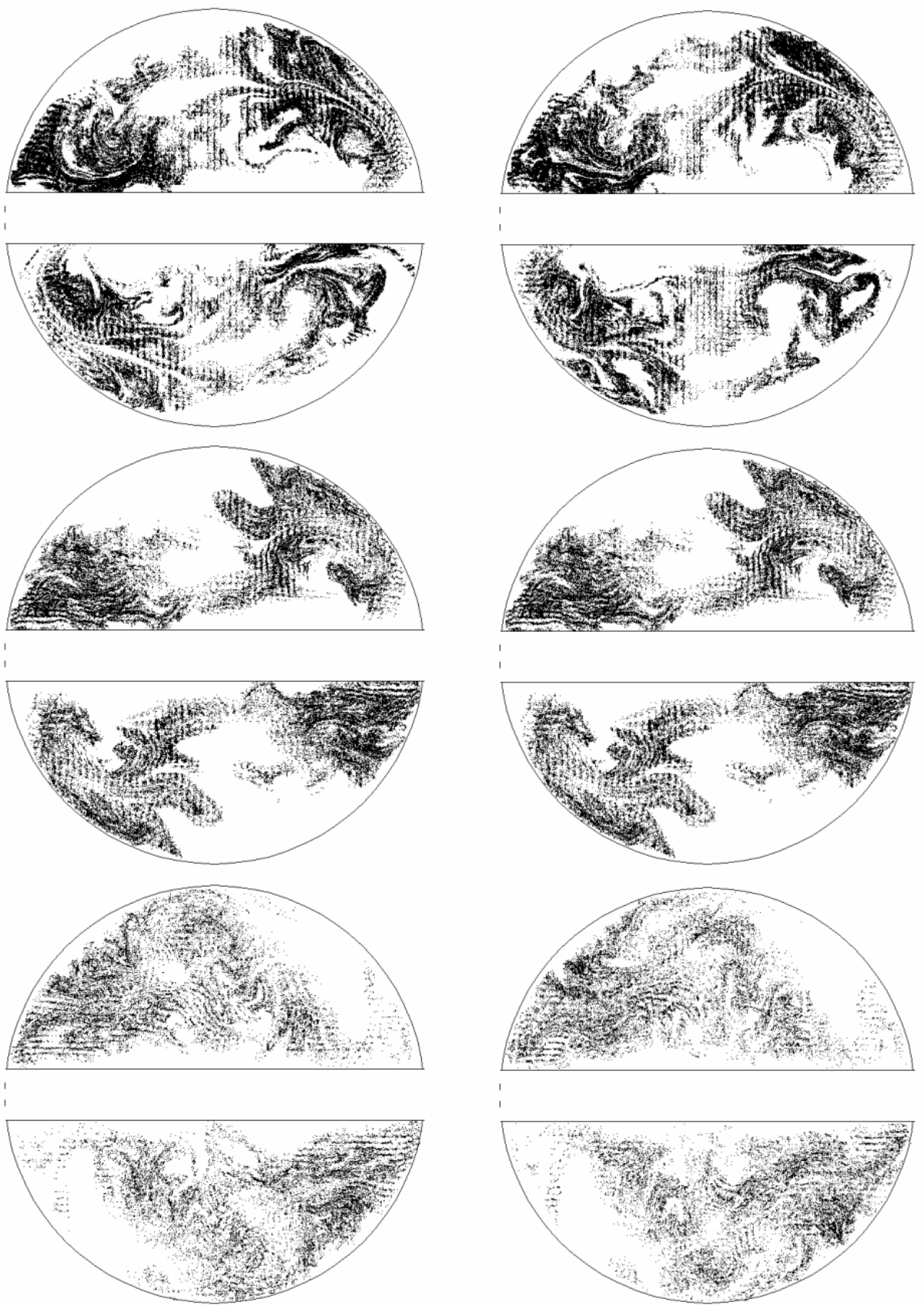

Figure 7.22: Particle locations the end of the 2nd, 4th, and 6th mixing elements, respectively from left to right (Left: at physical time $=0.7509 \mathrm{~s}$, Right: at physical time $=0.80546 s$ ) 


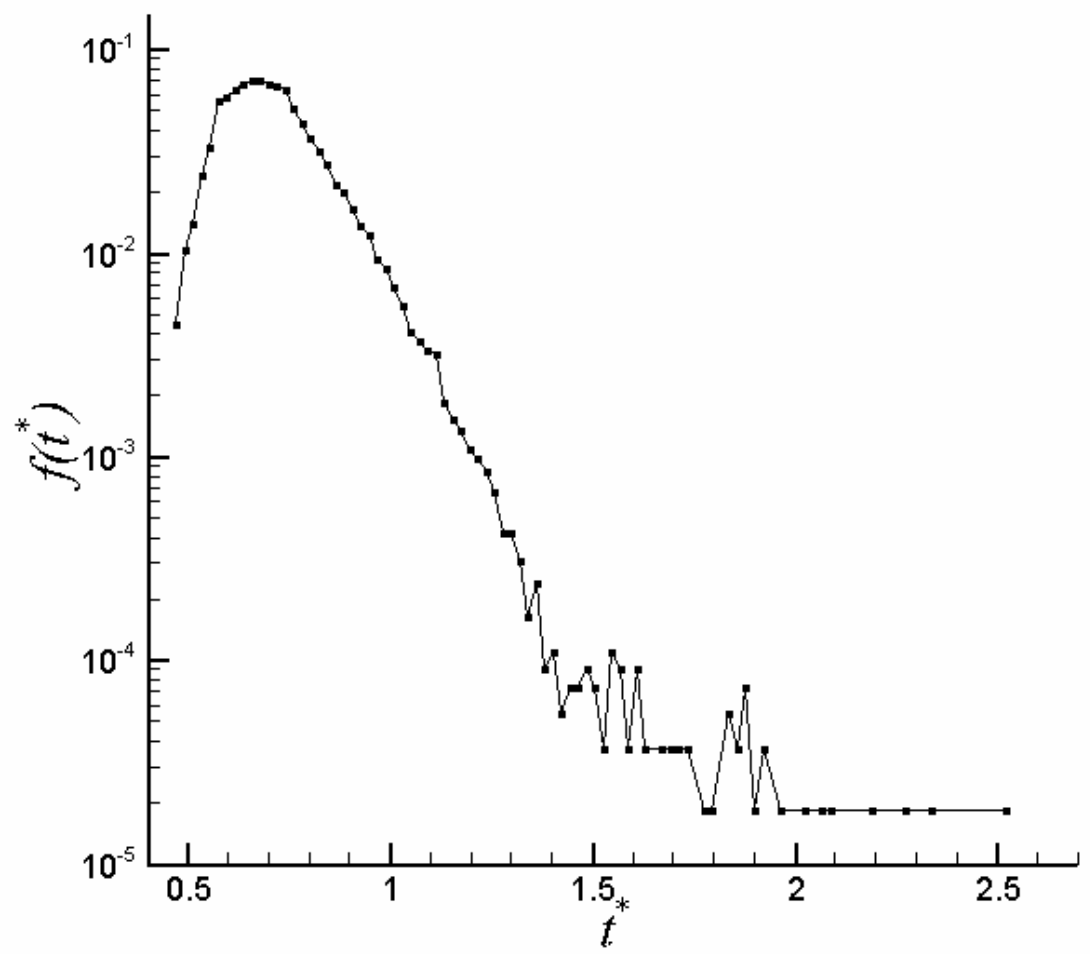

Figure 7.23: Distribution function for flow in the six-element helical static mixer 


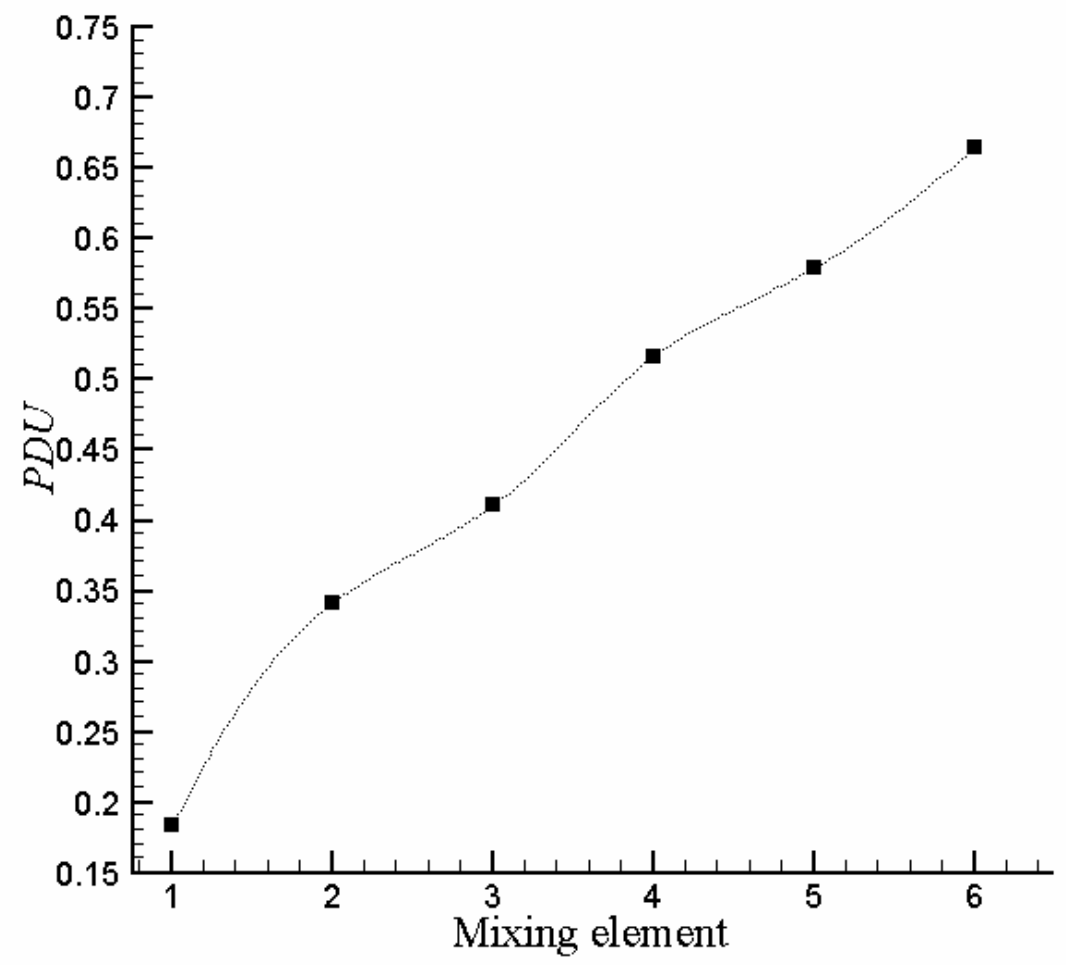

Figure 7.24: PDU values at the six mixing elements 


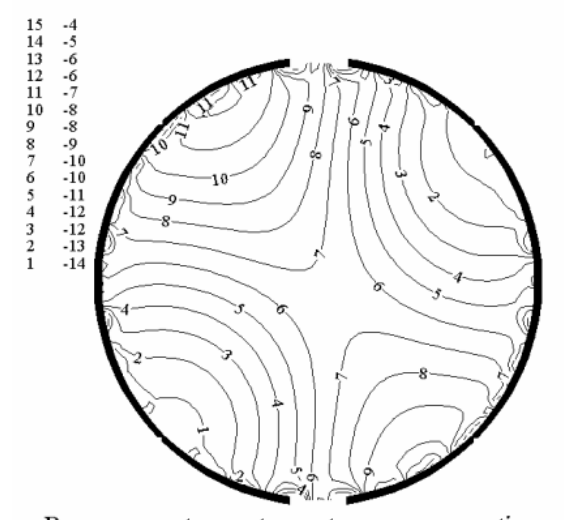

Pressure contours at up-stream cross-section

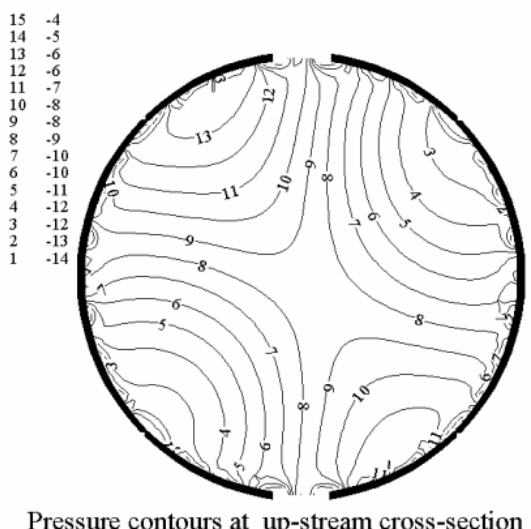

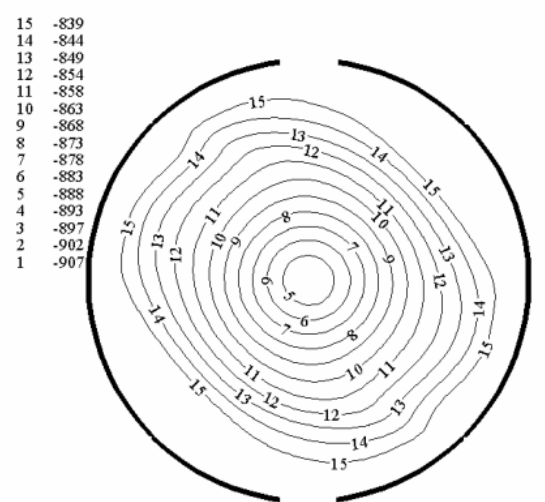

Pressure contours at down-stream cross-section

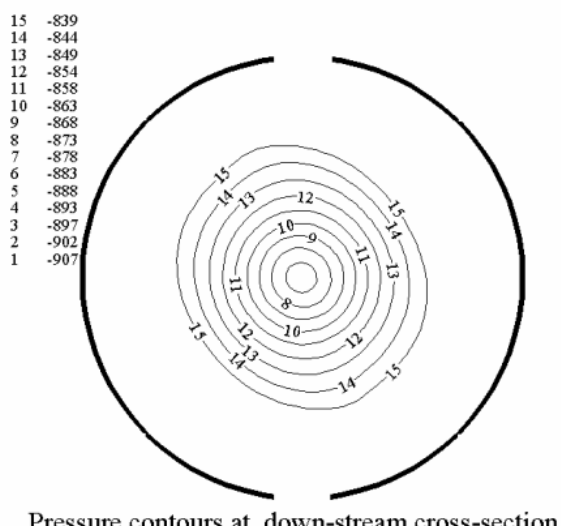

Figure 7.25: Static pressure at the upstream and the down stream of a six-element static mixer (Pa), predicted by $k-\omega$ (top) and RSM (bottom) models 


\section{CHAPTER EIGHT}

\section{HEAT TRANSFER RATE IN A HELICAL STATIC MIXER}

In chemical processing industries, heating, cooling and other thermal processing of viscous fluids are an integral part of the unit operations. Static mixers are often used in continuous mixing, heat transfer, and chemical reactions applications. In these processes, mechanical heat transfer predominantly occurs by screws or scraping vanes. But, thermal processing by conventional equipment with mechanically moving parts has several shortcomings, including abrasion, contamination, fluid leakage and abnormal stagnation of fluid. Furthermore, revolving scrapers may consume more energy and contaminate the product being processed with metallic tailings from the scrapings. Static mixers can be employed for those applications.

Because of the increased internal film coefficients and heat transfer rates provided by motionless mixer elements, heat exchanger designs utilizing static mixers can be smaller than conventional shell-tube heat exchangers. The helical insert causes a secondary flow in plane perpendicular to the predominant, axial flow. Compared to a tube of the same length and same diameter with no mixer, the helical static mixer is able to provide a substantial enhancement of heat and mass transfer from the surface of the tube to the 
fluid. Static mixer elements can reduce film buildup and improve heat transfer, allowing the reduction of heat exchanger size and space requirements. Static mixers can provide a more uniform temperature distribution in flowfield, and prevent the occurrence of local heating or cooling.

In this chapter heat transfer within a helical static mixer is investigated. Generally, heat transfer rate in a static mixer is a function of the physical properties of the working fluid, the flow conditions, and the geometrical parameters of mixer. Science in this study, the working fluid and the geometrical parameters are not changed, the heat transfer rate can only be related to the flow conditions. The effects of different flow conditions on the performance of the mixer are studied. Heat transfer rates for a flow in a pipe containing no mixer is compared to that with a helical static mixer.

\subsection{Numerical Method}

For a steady incompressible flow, the mass conservation and momentum equations are applied. Moreover, the conservation of energy is applied to flowfield. The energy equation for steady incompressible flow is

$$
\frac{\partial\left(\rho u_{i} E+p\right)}{\partial x_{i}}=\frac{\partial}{\partial x_{i}}\left(K \frac{\partial T}{\partial x_{i}}+u_{j} \tau_{i j}\right)
$$

The total energy per unit mass in Eq. (8.1) is 


$$
E=h-\frac{p}{\rho}+\frac{u_{i} u_{i}}{2}
$$

For a pure material with no phase change the enthalpy is given by

$$
h=\int_{0}^{T} c_{p} d T
$$

For the material used in this study, water, the value of density is $998.2 \mathrm{~kg} / \mathrm{m}^{3}$. Although the specific heat is a function of temperature, for the temperature range used in this study, its changes can be neglected; hence, specific heat is set at $4182 \mathrm{~J} / \mathrm{kg}$. K. However, for the thermal conductivity and the viscosity, the change of their values based on the temperature is significant and has an impact on the results of the numerical simulation. Therefore, the thermal conductivity and the viscosity are assumed piecewise linear functions of the temperature using the values from [71] shown in Table 8.1.

The numerical solver incorporates a segregated, implicit, second order upwind, double precision, finite volume approach. Using the segregated approach, the governing equations are solved sequentially. To obtain second-order accuracy, quantities at the cell faces are computed using a multidimensional linear reconstruction approach. The heat transfer in a six-element static mixer has been analyzed for a number of different conditions. A residual, less than $10^{-9}$ for temperature and less than $10^{-6}$ for other variables, defined as the L2 norm, were used as convergence criteria. 
Boundary Conditions: The fluid temperature at the inlet is set to $298.15 \mathrm{~K}\left(25^{\circ} \mathrm{C}\right)$ for all cases but one, which it is set to $278.15 \mathrm{~K}$. The pipe surface in the entrance and exit regions where there are no mixing elements is adiabatic. Two different boundary conditions are applied to the mixer surface: as the first boundary condition the mixer surface is considered adiabatic, which is called boundary condition (A); and as the second boundary condition, the mixer surface temperature is considered constant, which is called boundary condition $(\mathrm{T})$. These two boundary conditions on the mixer surface model two extreme limits for the mixer performance based on the thermal properties of a material that can be used to manufacture a helical static mixer: a material with no conductivity and a super conductive material. The pipe surface temperature in the region including the static mixer is set at various constants $(318.15 \mathrm{~K}, 348.15 \mathrm{~K}$, and $368.15 \mathrm{~K})$. No-slip boundary conditions are applied to the solid surface of the static mixer and also at the wall of the pipe. A constant mass flow rate is applied at the inlet and outlet boundaries. Different Reynolds numbers for the flow inside the pipe are obtained by varying the mass flow rate at the inlet boundary.

\subsection{Numerical Solution Accuracy}

The accuracy of the numerical solutions should be analyzed before confidence in the predictive ability of the numerical techniques can be justified. Accordingly, a detailed mesh convergence study was performed and indicated that the computational mesh employed in the present study is sufficiently refined to provide good numerical 
resolution. It is noted that $1,333,173$ mesh cells were used to compute the flow fields. The value of heat flux from the solid surfaces to the fluid, predicted by a computianal grid containing $2,211,841$ cells is only $0.447 \%$ more than the value of heat flux predicted by the grid with $1,333,173$ cells, when $\mathrm{Re}=100$.

The values of pressure drop across the mixer, obtained numerically, are found to be in good agreement with the experimental data. The computed values of Nusselt number for a fully developed flow in a long tube with no mixer shows good agreement with the experimental equation given by Seider and Tate [163]. The maximum error occurs for Re $=10$, at which the computed Nusselt number is 0.885 of the Nusselt number given by the Seider-Tate equation. The predicted values for Nusselt numbers for a pipe with static mixer shows agreement with the experimental data given in [34]. Figure 8.1 compares the Nusselt number obtained numerically, using boundary condition (T), to the existing experimental data for different flow Reynolds number. The maximum error occurs when $\operatorname{Re}=1$, which is about $19 \%$.

\subsection{Numerical Predictions}

The heat transfer and flow in a six-element static mixer has been analyzed for a number of different conditions. A residual of less than $10^{-9}$ for temperature and less than $10^{-6}$ for the other variables were used as the convergence criteria.

The temperature distribution and its gradient in a flow cross-section can have a critical role in the processing of materials. For flow Reynolds numbers of 1, 10, 100, 
1000 and different values of pipe wall temperature, contours of fluid temperature are obtained. For the case of $\operatorname{Re}=1$, the fluid temperature reaches the wall temperature shortly after entering the heated region of the pipe, applying ether boundary condition (A) or boundary condition (T).

For boundary condition (A), Figs. 8.2-8.4 show contours of temperature at the end of the second, the fourth, and the sixth mixing element, from top to bottom, respectively, for a pipe that contains a six-element static mixer. Figures 8.5-8.7 show the temperature contours at the same axial cross-sections in a pipe with no mixer. The pipe wall temperature is $318.15 \mathrm{~K}$. The fluid temperature at inlet is $298.15 \mathrm{~K}$.

For higher Reynolds numbers, the core flow remains at a low temperature in a pipe with no mixer; however, inserting a static mixer in the pipe breaks up the thermal boundary layer, leading to high temperature fluid particles. For $\operatorname{Re}=10$, after the fourth mixing element, the entire flow cross-section reaches the wall temperature. For the case of $\operatorname{Re}=100$, the area of the low temperature region is increased after the second element. This area continues to decrease as the flow passes through the helical static mixer. At the end of the sixth mixing element the area of the low temperature fluid is noticeably smaller when compared to the same flow cross-section for the case of a tube with no mixer in it. By increasing the Reynolds number, the difference of fluid emperature distribution in a cross-section between a tube with a static mixer and a tube with no mixer is more pronounced.

The averaged and the minimum fluid temperatures at different flow cross-sections for boundary condition (A) and different flow Reynolds numbers are shown in Tables 8.2 
and 8.3, respectively, for a pipe containing a six-element helical static mixer and a for pipe with no mixer. For the case of $\operatorname{Re}=1$, the averaged temperature and the minimum temperature are almost the same for both cases of a pipe with static mixer and a pipe with no mixer. These values are nearly equal to the wall temperature after the flow passes the fourth mixing element. For flow in a pipe with no mixer, when $\operatorname{Re}=10$, a large difference between the averaged and minimum values of fluid temperature is distinguishable in all cross-sections; however, for the flow in a pipe with static mixer, the difference between the averaged and minimum values of fluid temperature is considerably less, especially when the flow passes the sixth mixing element. The averaged temperature at the end of the sixth mixing element is $1.56 \mathrm{~K}$ less than the wall temperature, when the wall temperature is $318.15 \mathrm{~K}$ and it is $3.89 \mathrm{~K}$ less than the wall temperature when it is $348.15 \mathrm{~K}$; for the pipe with no mixer the averaged temperature at the same flow cross-section is $3.43 \mathrm{~K}(8.56 \mathrm{~K})$ less than the wall temperature, when the wall temperature is $318.15 \mathrm{~K}(348.15 \mathrm{~K})$. Although the differences between the averaged temperatures and the wall temperatures for the pipe with static mixer are less than those for the pipe with no mixer, the static mixer does not show a significant improvement. By increasing the flow Reynolds number these differences are more pronounced; for Re $=1,000$, the averaged temperature at the end of the sixth mixing element is $11.01 \mathrm{~K}$ $(27.53 \mathrm{~K})$ less than the wall temperature, when the wall temperature is $318.15 \mathrm{~K}(348.15$ $\mathrm{K})$. For the pipe with no mixer the averaged temperature at the same flow cross-section is $14.28 \mathrm{~K}(35.70 \mathrm{~K})$ less than the wall temperature, when the wall temperature is $318.15 \mathrm{~K}$ $(348.15 \mathrm{~K})$. 
The heat transfer coefficients for flow in a pipe that contains a six-element static mixer, with boundary condition (A), and for flow in a pipe with no mixer are compared in Figs. 8.8-8.10. The dashed-lines in these figures show the axial location of the contact point of each two mixing elements; and are numbered by the numbers of those elements. As can be seen, for the case of $\operatorname{Re}=1$, the heat transfer coefficients for both flows are the same; static mixer produces no increase in the heat transfer coefficient. When the Reynolds number is increased, the static mixer manifests a significant improve to the heat transfer coefficient. Also, it is seen that the heat transfer coefficient has local maximums around the contact point of each two mixing elements. Thus, in these transition areas the rate of heat transfer is higher.

The ratio of the minimum temperature to the averaged temperature was calculated at a cross-section located an $\mathrm{L} / 2$ distance downstream of the tailing edge of the last mixing element in a pipe with static mixer and at the same axial cross-section with a pipe with no mixer, and are shown in Table 8.4.

When the difference between the fluid temperature at inlet and the wall temperature is $20 \mathrm{~K}$, the static mixer produces almost no improvement in the temperature distribution. When the difference between the fluid temperature and the wall temperature is increased to $50 \mathrm{~K}$, the static mixer shows a better ability to develop a more uniform temperature distribution within the fluid, especially for the case of $\operatorname{Re}=100$. For $\operatorname{Re}=100$, in which the fluid temperature at inlet and the wall temperature are set to $278.15 \mathrm{~K}$ and $368.15 \mathrm{~K}$, respectively, the minimum and the averaged temperature for a pipe with static mixer at a cross-section located a distance L/2 downstream of the tailing edge of the last mixing 
element are $319.571 \mathrm{~K}$ and $324.838 \mathrm{~K}$, respectively. The minimum and the averaged temperature for a pipe with no mixer at the same axial cross-sections are $278.306 \mathrm{~K}$ and 313.564 K, respectively. When the difference between the fluid temperature at inlet and the wall temperature is increased to $90 \mathrm{~K}$, the static mixer manifests a higher performance. It increases the minimum and the averaged fluid temperatures significantly and also it decreases the temperature gradient in a flow cross-section significantly; the ratio of the minimum temperature to the averaged temperature is 0.984 for a pipe with static mixer, while it is only 0.888 for a pipe with no mixer.

The ratio of heat flux from the pipe wall into the fluid in a pipe with static mixer to the heat flux in a pipe with no mixer is presented at Table 8.5; two wall temperatures have been considered: $318.15 \mathrm{~K}$ and $348.15 \mathrm{~K}$. As expected, the wall temperature has no impact on the heat flux ratio.

For the case of $\operatorname{Re}=1$, the heat flux is slightly less when the pipe is equipped with static mixer. The area of the heated surface in a pipe with static mixer is $88.08 \%$ of the area of heated surface in a pipe with no mixer. This is because of the material thickness of the static mixer studied here. It is noticed that for a standard Kenics mixer, the mixer thickness is $4.17 \%$ of the mixer length; however, as mentioned before, for the TAH static mixer, this ratio is $22 \%$. In this flow condition, the static mixer is not able to bring a high degree of mixing to the fluid and therefore decreasing the heat transfer surface between the fluid and the heated surface leads to lower heat flux be transferred to the flowfield. By increasing the Reynolds number to 10 , the static mixer manifests a higher capability of fluid mixing, leading to higher convective heat exchange to the core fluid inside the 
tube and increases the rate of heat transfer to the flow. By increasing the Reynolds number from 10 to 100 , the heat flux ratio increases significantly ( 2.17 for $\operatorname{Re} 100$, compared to 1.22 for $\operatorname{Re}=10$ ). By increasing the Reynolds number to 1,000, again, the heat flux is increased, however, the rate of its increase is not as significant as for the case of $\operatorname{Re}=100$. In fact, when the Reynolds number is increased from 10 to 100 , the heat flux ratio is increased by $78.19 \%$, where as, when the Reynolds number is increased form 1 to 10 , the heat flux ratio is increased by $22.0 \%$ and when the Reynolds number is increased from 100 to 1000 , the heat flux ratio is increased by $26.18 \%$.

This can be explained by fluid mixing performance of the helical static mixer, studied in Chapter 5. By increasing the number of the mixing elements, the PDU value increases. When the flow regime changes from creeping flow to laminar flow, the rate of increase of the PDU values, is also increased. It can be seen that by increasing the Reynolds number from an order of $10^{1}$ to the order of $10^{2}$, the rate of increase of PDU values, based on the number of mixing elements, increases.

It worth mentioning that using the first order upwind method to solve the energy equation leads to an overestimation of the heat flux; for the case of $\operatorname{Re}=100$, first order upwind predicts a heat flux $17.92 \%$ higher in a pipe with a six-element mixer. Also it was observed that using a constant value for thermal conductivity leads to an underestimation of heat flux; for the case of $\operatorname{Re}=1,000$, a constant thermal conductivity assumption results in a heat flux, 3.3\% smaller in a pipe with a six-element mixer.

Figures 8.11 and 8.12 show contours of temperature at the end of the second, the fourth, and the sixth mixing element, from top to bottom, respectively, for a pipe that 
contains a six-element static mixer, applying boundary condition $(\mathrm{T})$. For $\operatorname{Re}=1$, the temperature at these flow cross-sections is equal to the wall temperature. For $\operatorname{Re}=10$, only temperature contours are distinguishable at the end of the second helical element; at the fourth and the sixth element the temperature gradient in the flow cross-section is extremely small and virtually all fluid elements are reach the wall temperature. For the case of $\operatorname{Re}=100$, the area of the low temperature region, at the end of the second mixing element, is significantly smaller compared to the results obtained for the same Reynolds number using $\mathrm{BC}(\mathrm{A})$. Also, the minimum temperature is higher for the case of $\mathrm{BC}(\mathrm{T})$, compared to the case of $\mathrm{BC}(\mathrm{A})$. The same pattern can be observed at the end of the fourth and the sixth helical mixing elements. By increasing the Reynolds number to 1,000 , the difference of fluid temperature distribution in a cross-section between a static mixer with $\mathrm{BC}(\mathrm{A})$ and static mixer with $\mathrm{BC}(\mathrm{T})$ is more pronounced.

Applying boundary condition (T) to the mixer surface, the flowfield was studied for different flow conditions. Averaged and the minimum fluid temperatures at different flow cross-section are presented in Table 8.6. The minimum to averaged temperature ratios at the end of even numbered mixing element for different flow Reynolds number are given in Table 8.7. For $\operatorname{Re}=1$ and $\operatorname{Re}=10$, the averaged temperature and the minimum temperature are almost the same for both cases of a pipe with static mixer and a pipe with no mixer. These values are nearly equal to the wall temperature after the flow passes the fourth mixing element. For flow in a pipe with no mixer, for $\operatorname{Re}=100$ and $\operatorname{Re}=1000$, a large difference between the averaged and minimum values of fluid temperature is distinguishable in all cross-sections; however, for the flow in a pipe with static mixer, the 
difference between the averaged and minimum values of fluid temperature is considerably less, especially when the flow passes the sixth mixing element. Comparing these results, obtained by applying boundary condition (T), to the results obtained by applying boundary condition (A), shows that the mixer has a higher performance under boundary condition (T), especially for higher Reynolds numbers.

Table 8.8 presents the maximum to minimum temperature ratios at the end of each even numbered element, for $\operatorname{Re}=1000$. When Reynolds number is small, this ratio is one at all flow cross-sections. For higher Reynolds numbers this ratio increases when the wall temperature is increased. As the flow passes through the mixing elements the ratio decreases and therefore fluid temperature is more uniform. The rate of decrease of the temperature ratio is higher when the wall temperature is higher, indicating that the helical static mixer has better performance for such cases.

Table 8.9 shows the heat flux ratio, which is the heat flux obtained by applying boundary $(\mathrm{T})$, divided by the heat flux obtained by applying boundary condition (A). For low Reynolds number flows, this ratio is almost one. For such a case the static mixer material has a minimal impact on the rate of heat transfer. The heat flux ratio increases about $10 \%$, when Reynolds number increases from 1 to 10 . For $\operatorname{Re}=100$, the rate of heat transfer to the working fluid is about $60 \%$ greater, when the mixer surface has a temperature equal to the pipe wall. When Reynolds number increases form 100 to 1,000, again the heat flux ratio increases, although the rate of increase is not as high as the one when Reynolds number increased from 10 to 100. 
Table 8.10 presents TDR values at the end of the last mixing element for different flow Reynolds numbers. When $\operatorname{Re}=1$, TDR is equal to zero for boundary condition (A). For such a case, TDR is 1.0 for boundary condition (T); as it was mentioned in Chapter 4, in this situation the TDR value cannot reveal any information about the mixer performance. For the case studied here, the difference between maximum and minimum temperatures for a pipe with no mixer is only $0.005 \mathrm{~K}$. Therefore, the static mixer does not improve the temperature blending process in this case either. When $\operatorname{Re}=10, \mathrm{TDR}=$ 0.6837, for boundary condition (A). By increasing the Reynolds number, TDR values decrease. This suggests that the helical static mixer is more effective for low Reynolds number laminar flows. When boundary condition (T) is applied, the TDR value is high for all Reynolds numbers from 10 to 1,000. Using a heat conductive material for the static mixer enhances the temperature blending process significantly in laminar flows. 


\begin{tabular}{|c|c|c|}
\hline $\begin{array}{c}\text { Temperature } \\
(K)\end{array}$ & $\begin{array}{c}\text { Thermal conductivity } \\
(\mathrm{W} / \mathrm{m} . \mathrm{K})\end{array}$ & $\begin{array}{c}\text { Viscosity } \\
(\mathrm{Kg} / \mathrm{m} . \mathrm{s})\end{array}$ \\
\hline 273.15 & 0.564 & 0.001792 \\
\hline 278.15 & 0.574 & 0.001519 \\
\hline 283.15 & 0.584 & 0.001307 \\
\hline 288.15 & 0.593 & 0.001138 \\
\hline 293.15 & 0.602 & 0.001002 \\
\hline 298.15 & 0.610 & 0.000890 \\
\hline 303.15 & 0.617 & 0.000797 \\
\hline 308.15 & 0.624 & 0.000719 \\
\hline 313.15 & 0.631 & 0.000653 \\
\hline 318.15 & 0.637 & 0.000596 \\
\hline 323.15 & 0.643 & 0.000547 \\
\hline 328.15 & 0.648 & 0.000504 \\
\hline 333.15 & 0.653 & 0.000467 \\
\hline 338.15 & 0.657 & 0.000434 \\
\hline 343.15 & 0.662 & 0.000405 \\
\hline 348.15 & 0.665 & 0.000379 \\
\hline 353.15 & 0.669 & 0.000355 \\
\hline 358.15 & 0.673 & 0.000334 \\
\hline 363.15 & 0.676 & 0.000315 \\
\hline 368.15 & 0.678 & 0.000298 \\
\hline 373.15 & 0.681 & 0.000282 \\
\hline
\end{tabular}

Table 8.1: Values of thermal conductivity and viscosity for water 


\begin{tabular}{|c|c|c|c|}
\hline Re & $\begin{array}{c}\text { Element } \\
\text { number }\end{array}$ & $\begin{array}{c}\text { Avg. T } \\
(K)\end{array}$ & $\begin{array}{c}\text { Min. T } \\
(K)\end{array}$ \\
\hline 1 & 2 & 317.978 & 317.747 \\
\hline 1 & 4 & 318.148 & 318.144 \\
\hline 1 & 6 & 318.148 & 318.145 \\
\hline 10 & 2 & 311.692 & 304.653 \\
\hline 10 & 4 & 315.110 & 311.990 \\
\hline 10 & 6 & 316.594 & 315.204 \\
\hline 100 & 2 & 307.304 & 299.511 \\
\hline 100 & 4 & 309.255 & 303.523 \\
\hline 100 & 6 & 310.357 & 306.624 \\
\hline 1000 & 2 & 303.383 & 299.620 \\
\hline 1000 & 4 & 305.519 & 302.675 \\
\hline 1000 & 6 & 307.139 & 304.939 \\
\hline
\end{tabular}

Table 8.2: Averaged and minimum fluid temperatures for a pipe with static mixer (wall temperature $=318.15 \mathrm{~K}, \mathrm{BC}(\mathrm{A})$ ) 


\begin{tabular}{|c|c|c|c|}
\hline Re & $\begin{array}{c}\text { Cross } \\
\text { section }\end{array}$ & $\begin{array}{c}\text { Avg. T } \\
(K)\end{array}$ & $\begin{array}{c}\text { Min. T } \\
(K)\end{array}$ \\
\hline 1 & 2 & 317.835 & 317.260 \\
\hline 1 & 4 & 318.137 & 318.113 \\
\hline 1 & 6 & 318.148 & 318.147 \\
\hline 10 & 2 & 310.872 & 299.659 \\
\hline 10 & 4 & 313.282 & 304.639 \\
\hline 10 & 6 & 314.725 & 308.811 \\
\hline 100 & 2 & 305.415 & 298.150 \\
\hline 100 & 4 & 306.774 & 298.151 \\
\hline 100 & 6 & 307.603 & 298.173 \\
\hline 1000 & 2 & 302.104 & 298.150 \\
\hline 1000 & 4 & 303.306 & 298.155 \\
\hline 1000 & 6 & 303.869 & 298.222 \\
\hline
\end{tabular}

Table 8.3: Averaged and minimum fluid temperatures for a pipe with no mixer (wall temperature $=\mathbf{3 1 8 . 1 5} \mathrm{K}$ ) 


\begin{tabular}{|c|c|c|c|}
\hline & $\operatorname{Re}$ & $\begin{array}{c}\text { Temp. ratio } \\
\mathrm{T}_{\mathrm{W}}=318.15 \mathrm{~K}\end{array}$ & $\begin{array}{c}\text { Temp. ratio } \\
\mathrm{T}_{\mathrm{W}}=348.15 \mathrm{~K}\end{array}$ \\
\hline \multirow{3}{*}{ 莕 } & 10 & 0.998 & 0.995 \\
\hline & 100 & 0.996 & 0.991 \\
\hline & 1000 & 0.999 & 0.997 \\
\hline \multirow{3}{*}{$\stackrel{\circ}{Z} \cdot \stackrel{\bar{x}}{\Xi}$} & 10 & 0.987 & 0.969 \\
\hline & 100 & 0.975 & 0.940 \\
\hline & 1000 & 0.988 & 0.971 \\
\hline
\end{tabular}

Table 8.4: Minimum to averaged temperature ratio (BC (A)) 


\begin{tabular}{|c|c|c|}
\hline Re & $\begin{array}{c}\text { Heat flux ratio } \\
\mathrm{T}_{\mathrm{W}}=318.15 \mathrm{~K}\end{array}$ & $\begin{array}{c}\text { Heat flux ratio } \\
\mathrm{T}_{\mathrm{W}}=348.15 \mathrm{~K}\end{array}$ \\
\hline 1 & 0.9988 & 0.9990 \\
\hline 10 & 1.2187 & 1.2187 \\
\hline 100 & 2.1716 & 2.1716 \\
\hline 1000 & 2.7401 & 2.7401 \\
\hline
\end{tabular}

Table 8.5: Heat flux ratio (BC (A)) 


\begin{tabular}{|c|c|c|c|}
\hline Re & $\begin{array}{c}\text { Element } \\
\text { number }\end{array}$ & $\begin{array}{c}\text { Avg. T } \\
(K)\end{array}$ & $\begin{array}{c}\text { Min. T } \\
(K)\end{array}$ \\
\hline 1 & 2 & 318.150 & 318.150 \\
\hline 1 & 4 & 318.150 & 318.150 \\
\hline 1 & 6 & 318.150 & 318.150 \\
\hline 10 & 2 & 317.22 & 312.739 \\
\hline 10 & 4 & 318.021 & 317.412 \\
\hline 10 & 6 & 318.130 & 318.046 \\
\hline 100 & 2 & 313.781 & 301.774 \\
\hline 100 & 4 & 315.595 & 309.641 \\
\hline 100 & 6 & 316.434 & 313.300 \\
\hline 1000 & 2 & 311.113 & 302.352 \\
\hline 1000 & 4 & 313.159 & 307.098 \\
\hline 1000 & 6 & 314.359 & 310.600 \\
\hline
\end{tabular}

Table 8.6: Averaged and minimum fluid temperatures for a pipe with static mixer $($ wall temperature $=318.15 \mathrm{~K}, \mathrm{BC}(\mathrm{T})$ ) 


\begin{tabular}{|c|c|c|c|}
\hline $\operatorname{Re}$ & $\begin{array}{c}\text { Temp. ratio } \\
\text { 2nd element }\end{array}$ & $\begin{array}{c}\text { Temp. ratio } \\
\text { 4th element }\end{array}$ & $\begin{array}{c}\text { Temp. ratio } \\
\text { 6th element }\end{array}$ \\
\hline 10 & 0.985868086850256 & 0.998085073479070 & 0.999736642731888 \\
\hline 100 & 0.961733140262416 & 0.981133927527240 & 0.990097203222500 \\
\hline 1000 & 0.971839517288645 & 0.980646703285024 & 0.988045926597824 \\
\hline
\end{tabular}

Table 8.7: Minimum to averaged temperature ratio $\left(\mathrm{T}_{\mathrm{W}}=318.15 \mathrm{~K}, \mathrm{BC}(\mathrm{T})\right)$ 


\begin{tabular}{|c|c|c|c|}
\hline $\begin{array}{c}\mathrm{T}_{\mathrm{W}} \\
(K)\end{array}$ & $\begin{array}{c}\text { Temp. ratio } \\
\text { 2nd element }\end{array}$ & $\begin{array}{c}\text { Temp. ratio } \\
\text { 4th element }\end{array}$ & $\begin{array}{c}\text { Temp. ratio } \\
\text { 6th element }\end{array}$ \\
\hline 318.15 & 1.052 & 1.036 & 1.024 \\
\hline 348.15 & 1.128 & 1.086 & 1.057 \\
\hline 368.15 & 1.177 & 1.117 & 1.077 \\
\hline
\end{tabular}

Table 8.8: Maximum to minimum temperature ratio $(\operatorname{Re}=1000, \mathrm{BC}(\mathrm{T}))$ 


\begin{tabular}{|c|c|}
\hline $\operatorname{Re}$ & Heat flux ratio \\
\hline 1 & 1.0033 \\
\hline 10 & 1.1073 \\
\hline 100 & 1.5866 \\
\hline 1000 & 1.7047 \\
\hline
\end{tabular}

Table 8.9: Ratio of heat flux with BC (T) to heat flux with BC (A) 


\begin{tabular}{|c|c|c|}
\hline $\operatorname{Re}$ & $\begin{array}{c}\text { TDR } \\
(\mathrm{BC}(\mathrm{A}))\end{array}$ & $\begin{array}{c}\text { TDR } \\
(\mathrm{BC}(\mathrm{T}))\end{array}$ \\
\hline 1 & 0.0000 & 1.0000 \\
\hline 10 & 0.6837 & 0.9884 \\
\hline 100 & 0.4413 & 0.9707 \\
\hline 1000 & 0.3961 & 0.9585 \\
\hline
\end{tabular}

Table 8.10: Temperature Difference Ratio $\left(T_{W}=348.15 K\right)$ 


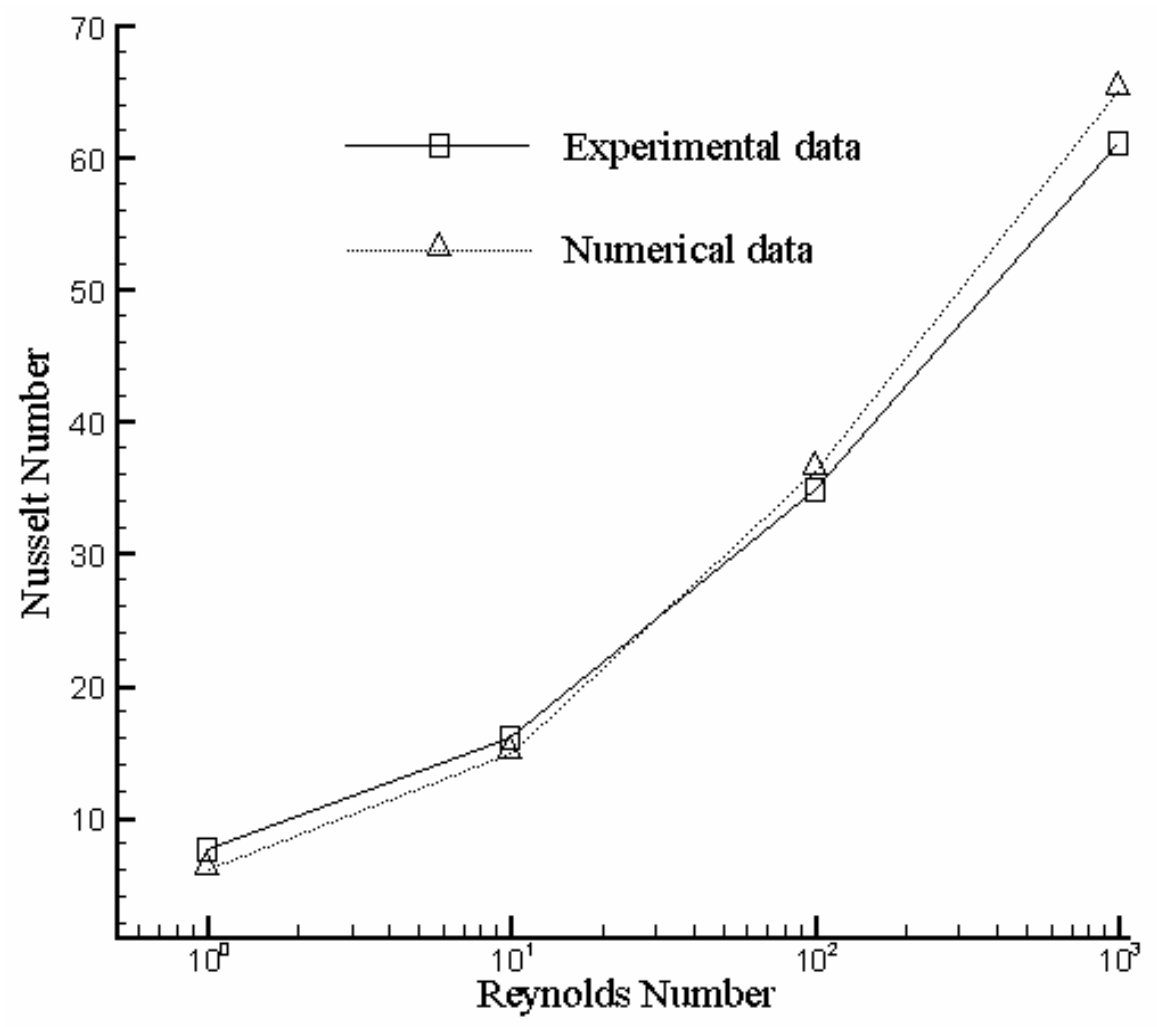

Figure 8.1: Comparison between numerical and experimental data 

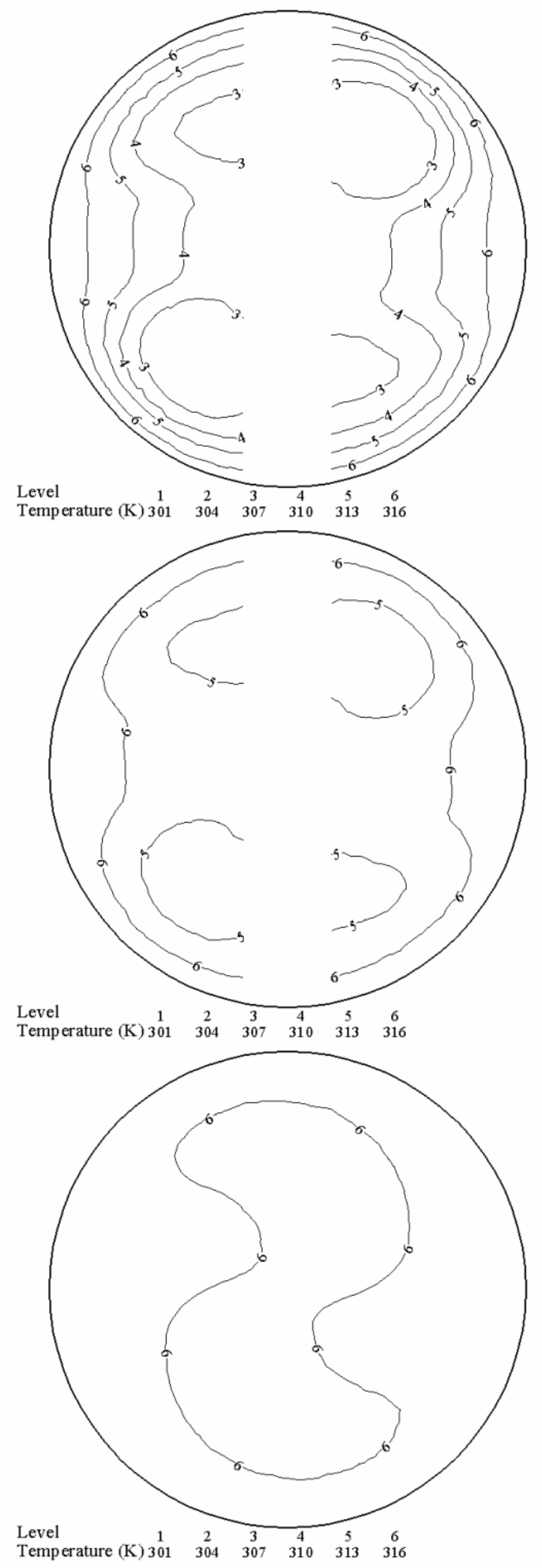

Figure 8.2: Temperature at even numbered elements, $\operatorname{Re}=10$ 

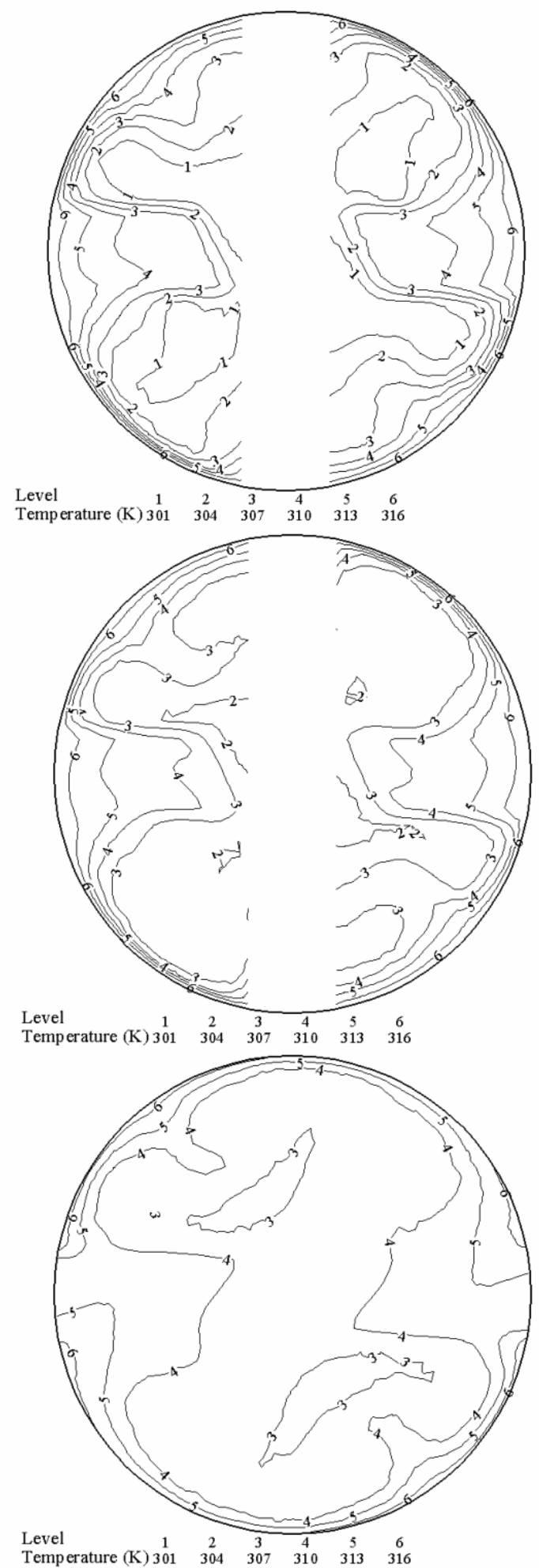

Figure 8.3: Temperature at even numbered elements, $\operatorname{Re}=100$ 


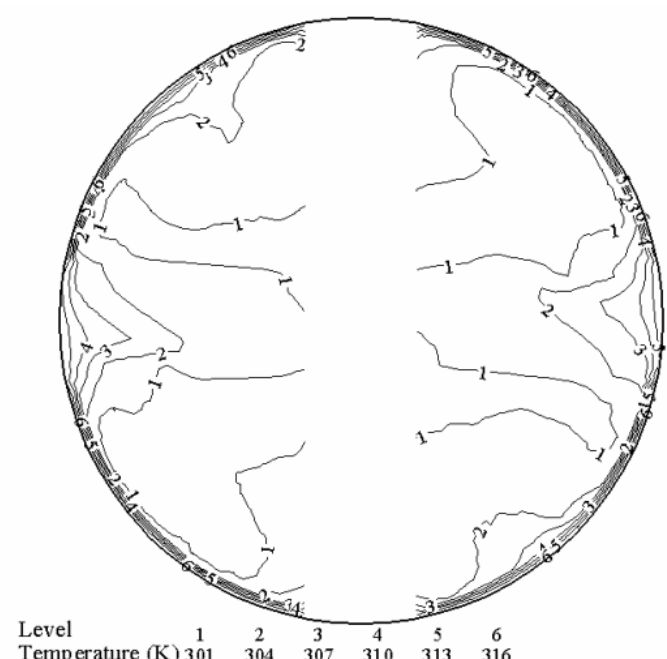

Temperature (K) $301 \quad 304 \quad 307 \quad 310 \quad 313 \quad 316$

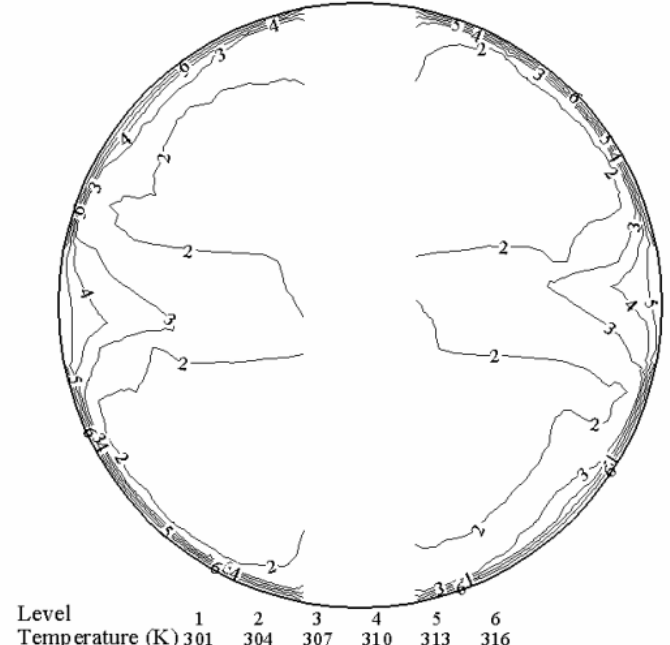

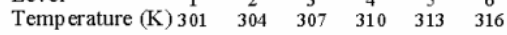

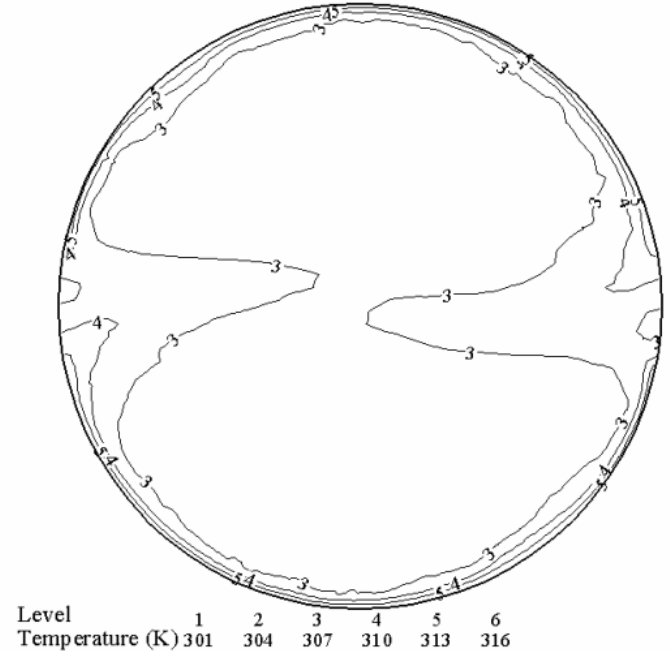

Figure 8.4: Temperature at even numbered elements, $\operatorname{Re}=1000$ 


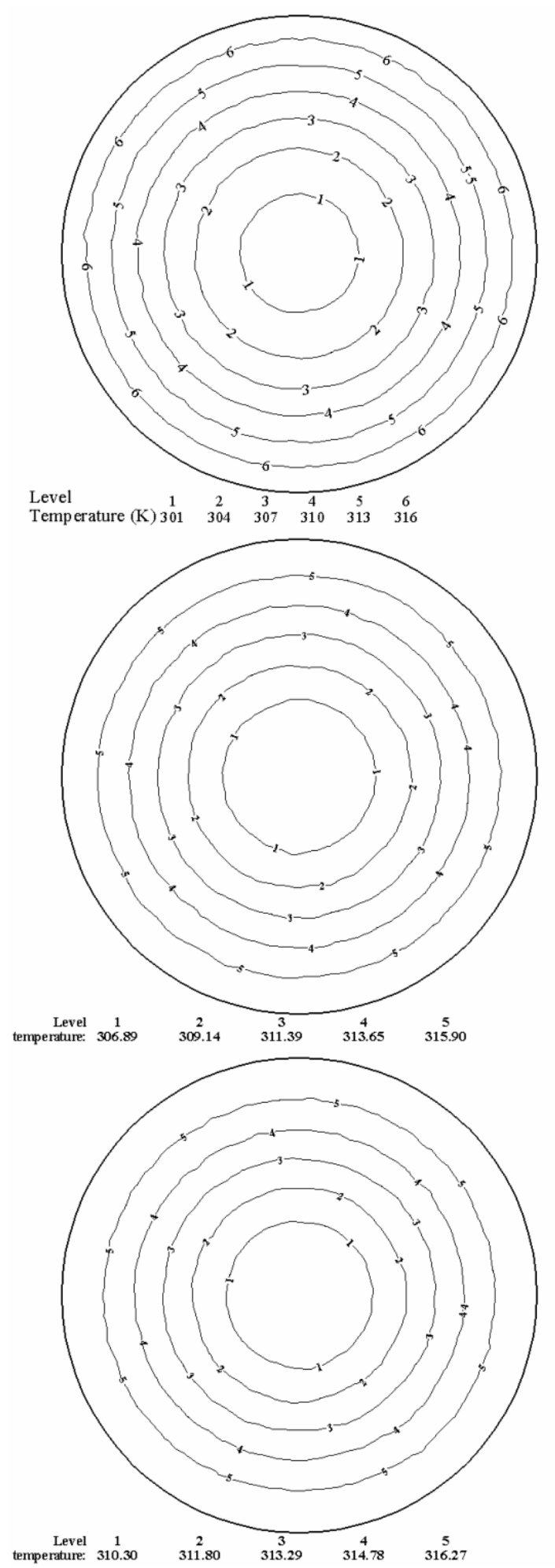

Figure 8.5: Temperature contours at the same cross-sections, $\operatorname{Re}=10$ 

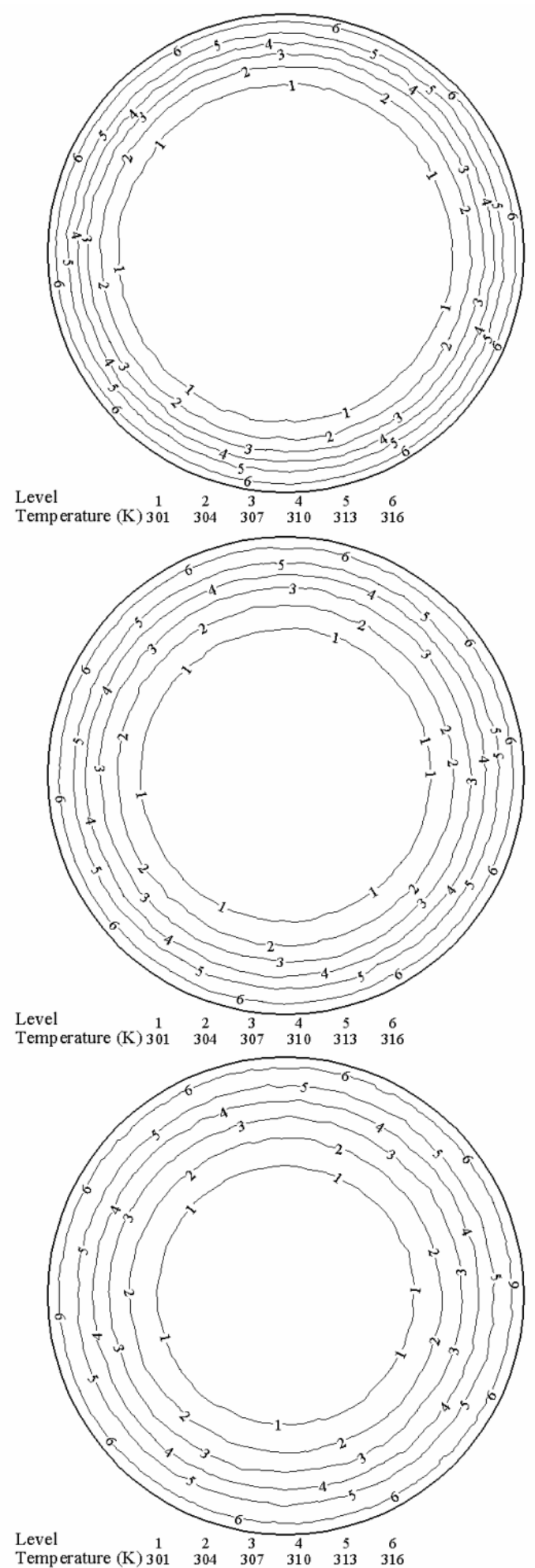

Figure 8.6: Temperature contours at the same cross-sections, $\operatorname{Re}=100$ 

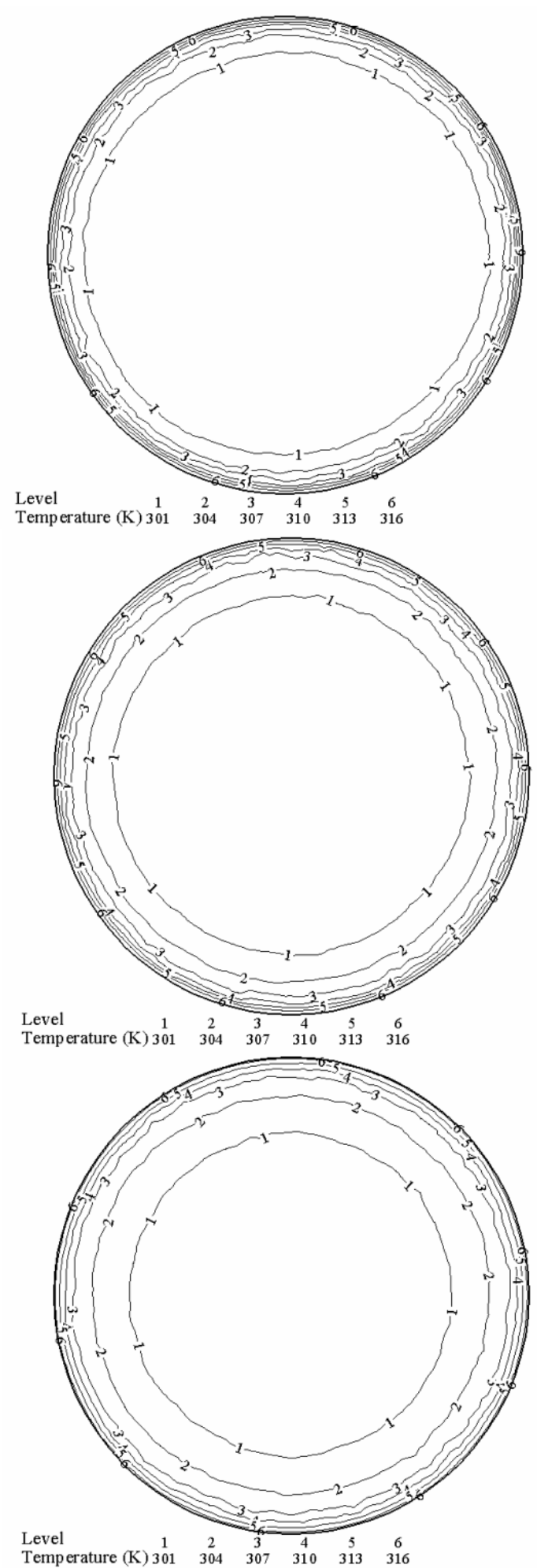

Figure 8.7: Temperature contours at the same cross-sections, $\operatorname{Re}=1000$ 
$\operatorname{Re}=1$

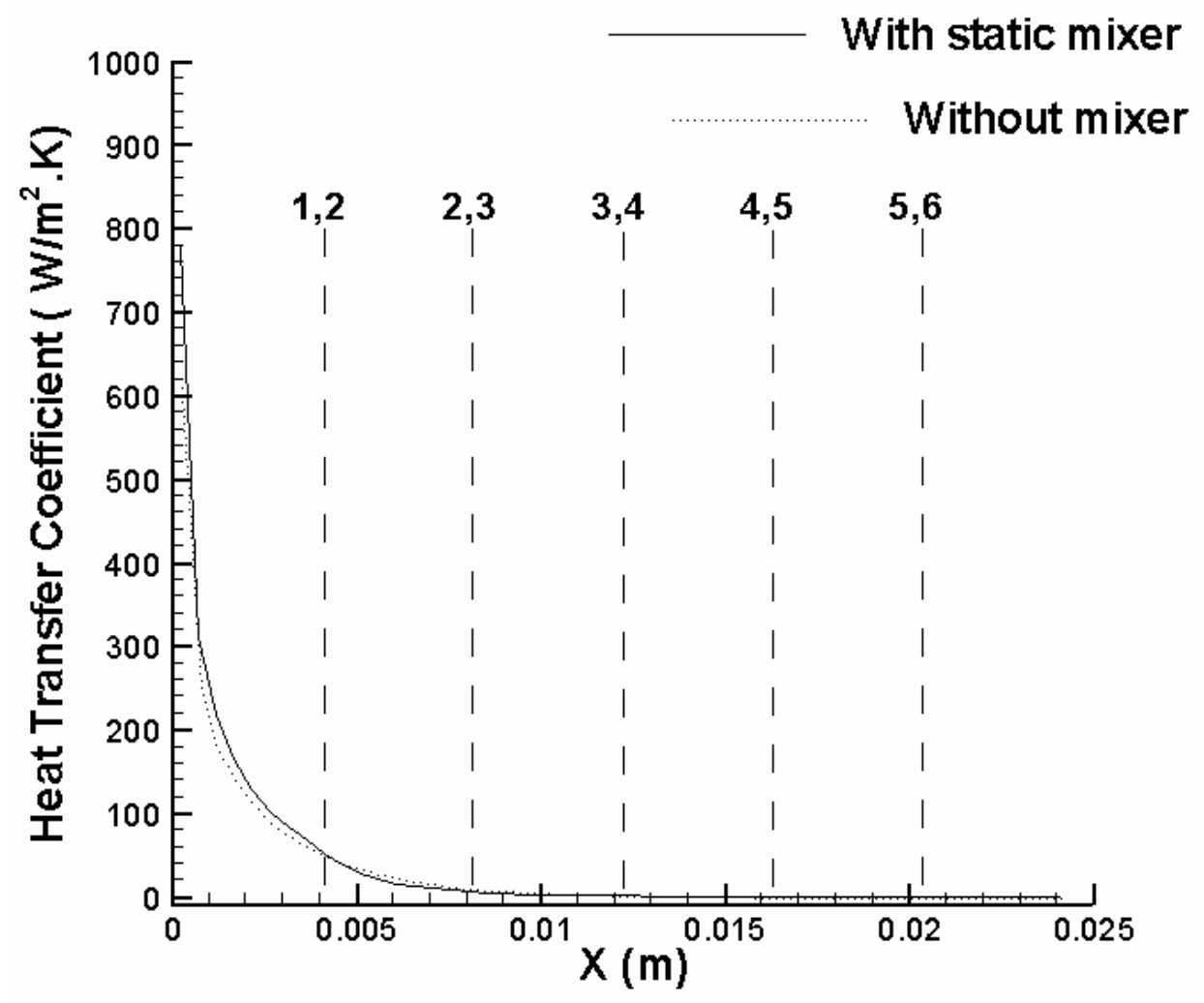

Figure 8.8: Heat transfer coefficient in a pipe $(\operatorname{Re}=1, B C(A))$ 
$\operatorname{Re}=100$

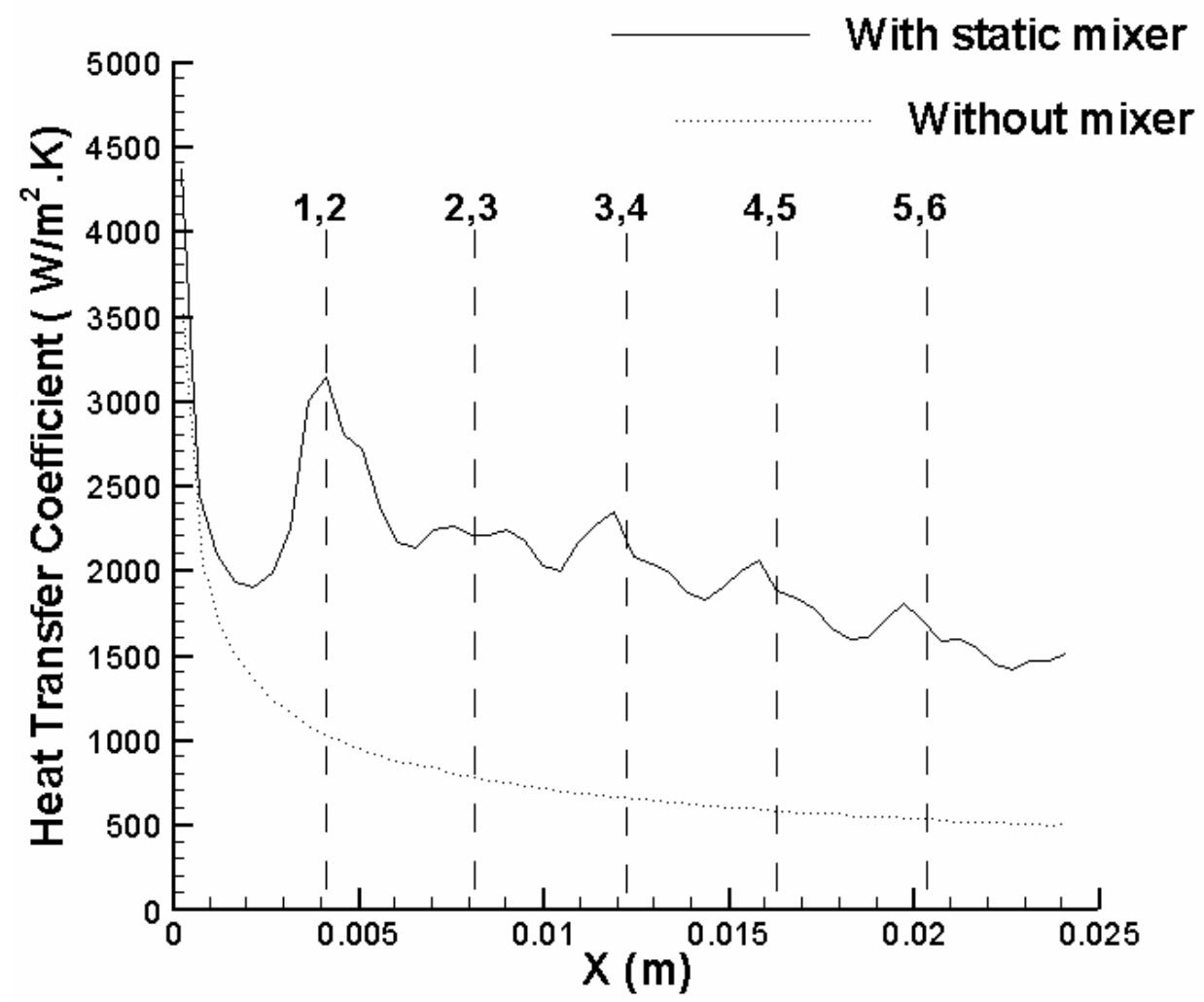

Figure 8.9: Heat transfer coefficient in a pipe $(\operatorname{Re}=100, \mathrm{BC}(\mathrm{A}))$ 


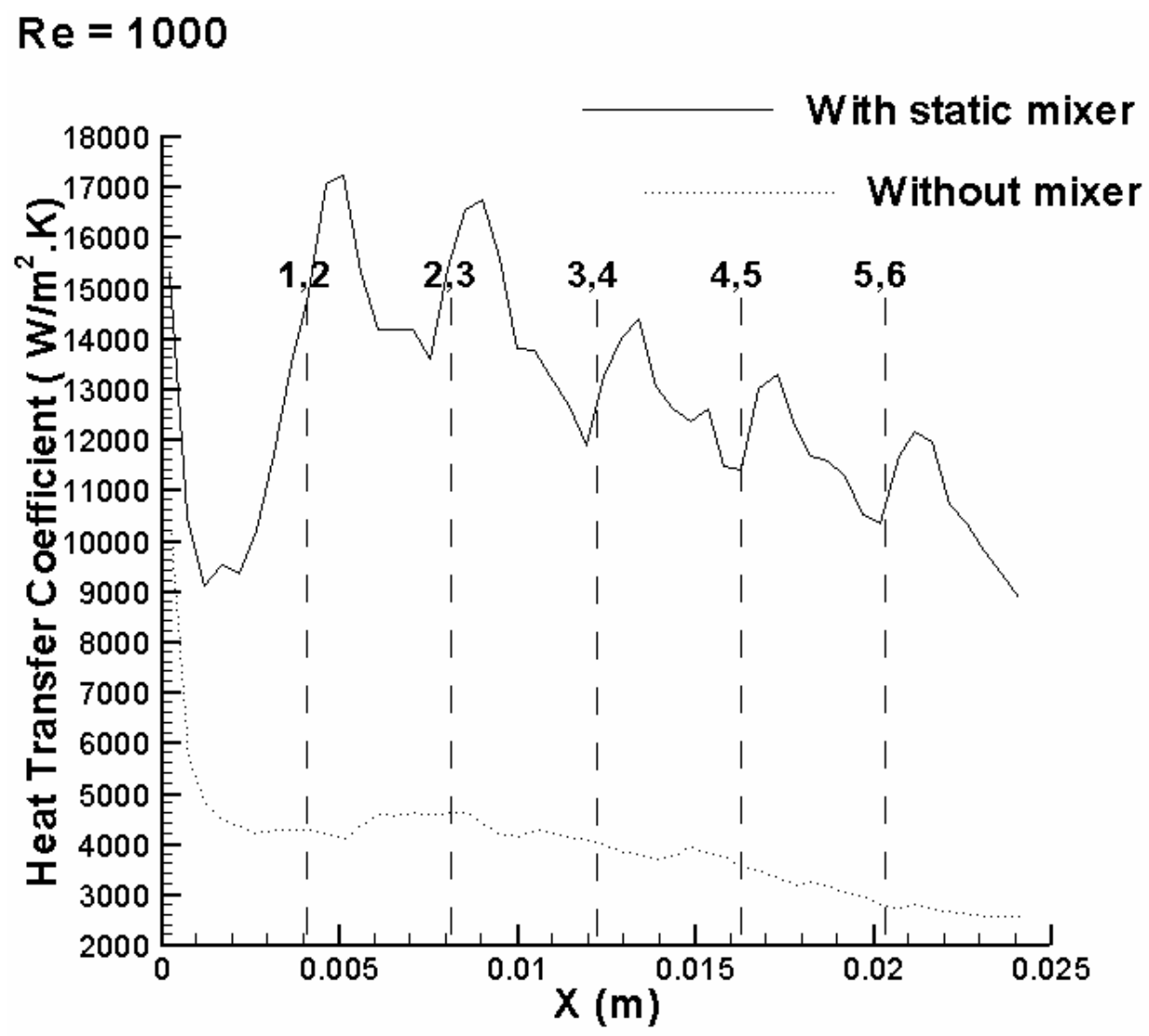

Figure 8.10: Heat transfer coefficient in a pipe $(\operatorname{Re}=1000, B C(A))$ 


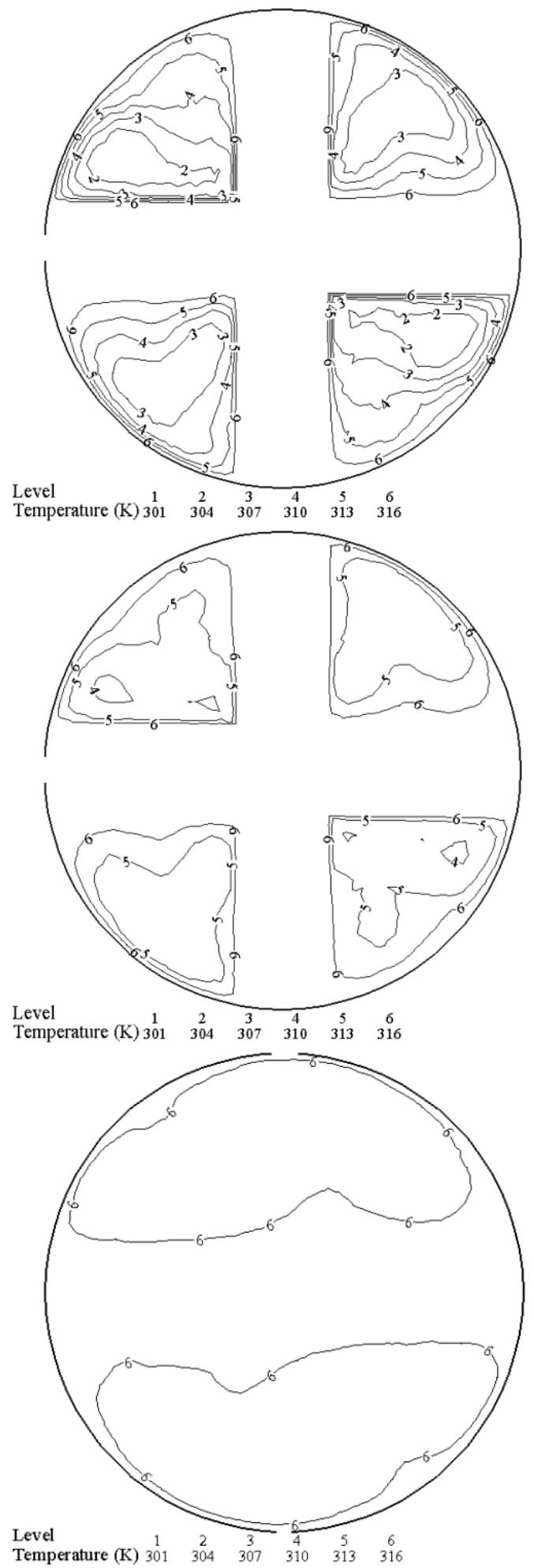

Figure 8.11: Temperature contours, $B C(T), \operatorname{Re}=100$ 


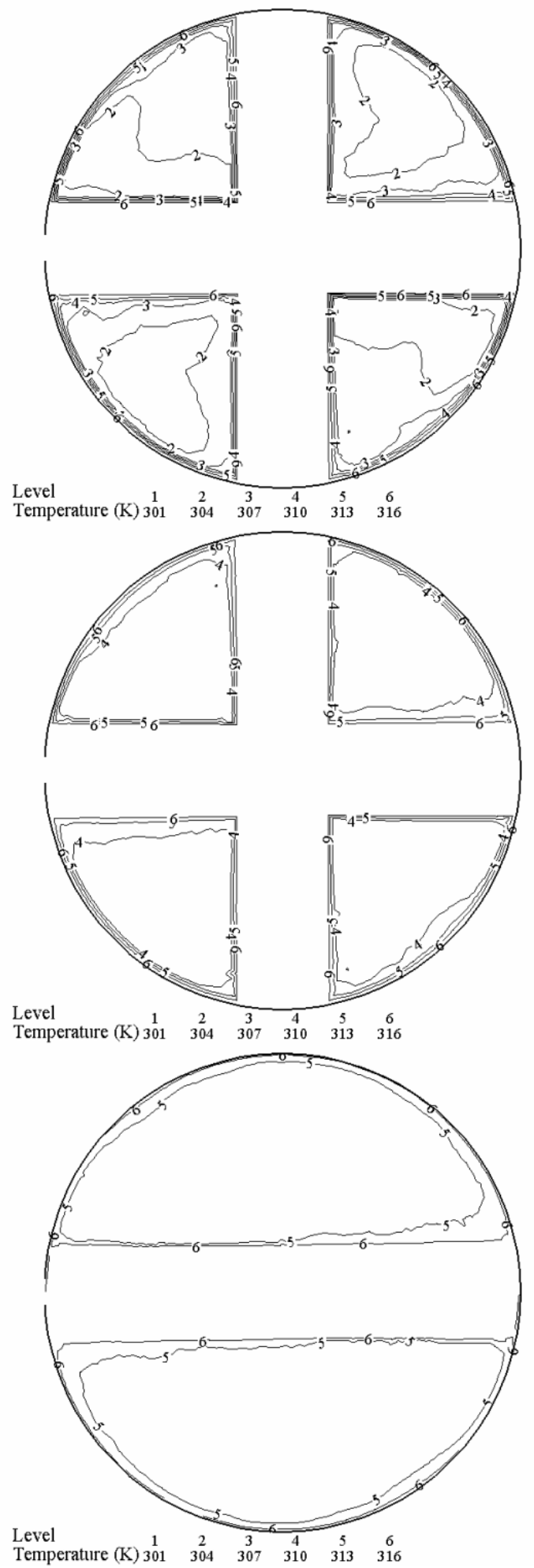

Figure 8.12: Temperature contours, $\mathrm{BC}(\mathrm{T}), \mathrm{Re}=1000$ 


\section{CONCLUSIONS}

Performance of a compact industrial helical static mixer, for a variety of flow conditions, was studied efficiently on a PC. A second order accurate numerical method was used. Comparison with certain experimental values of the pressure drop across the mixer measured for the standard ele ment twist angle shows very good agreement over a range of flow conditions. Also, the numerically predicted Nusselt numbers are in good agreement with the experimental data for different flow Reynolds numbers.

- Study of a helical static mixer in laminar flow shows that the Reynolds number has a major impact on the performance of the mixer. For low Reynolds number flows most of fluid particles are separated, whereas for higher Reynolds number flows increased mixing of fluid particles occurs.

The distribution functions are similar for Reynolds numbers up to 100 . However, for $\operatorname{Re}=1,000$, the distribution function is quite different. The maximum nondimensional residence time is more than 3 for all cases studied except for $\operatorname{Re}=$ 1,000 , which yields a value about 1.8 .

By increasing the Reynolds number, the structure radius might be expected to be decreased. Although this is true for most cases, it is not correct in general. For Re $=10$, the radius structure at the last mixing element is higher compared to the radius structure at the same flow cross-section, when $\mathrm{Re}=1$ or less.

PDU value increases by increasing the number of the mixing elements. By changing the flow pattern from creeping flow to laminar flow, the rate of increase 
of the PDU values is also increased. For the case of $\mathrm{Re}=10$, PDU value decreases at the end of the last mixing element.

- Performance of helical static mixer using pseudo-plastic fluids was studied. It was observed that the power law model can lead to unrealistic results in the study of static mixer performance. The Carreau model can provide accurate results. The Reynolds number and concentration level of the non-Newtonian solution have a major impact on the performance of the helical static mixer. For low Reynolds number flows, most of the fluid particles are separated. For higher Reynolds number flows, increased mixing of fluid particles occurs. Pressure drop across the static mixer is affected by the concentration level of a CMC solution. Dilute CMC solutions leads to a very low pressure drop. The pressure drop increases significantly with increasing concentration level. The mixed fluid pattern, however, is not affected by the concentration level of CMC solution.

The PDU values for creeping flow conditions are very close and almost the same. Increasing the modified Reynolds number increases the PDU values. A significant increase in PDU values can be seen when the modified Reynolds number increases to 100; by increasing the modified Reynolds number from 100 to 1000 . It was found that for the same flow Reynolds number, the helical static mixer has different performance for Newtonian and shear thinning non-Newtonian fluids, pseudo-plastic fluids, in non-creeping flows. For low Reynolds number flows, when the working fluid is non-Newtonian, static mixer efficiency is more than two times of the mixer efficiency, when the working fluid is Newtonian. For 
higher Reynolds numbers, the superiority of the static mixer with non-Newtonian fluids to the mixer with Newtonian fluids is less pronounced; for Reynolds number of 1,000 , the helical static mixer efficiency with non-Newtonian fluid is about $15 \%$ more than the static mixer efficiency with Newtonian fluid.

- The accuracy, global performance, and computational expenses of four RANS turbulence models, the Spalart-Allmaras, the $k-\varepsilon$, the $k-\varepsilon k-\omega$ model and the RSM model, for flow in an industrial helical static mixer was numerically investigated. Also, the global performance of static mixer in turbulent flow regime was studied by the LES model.

As long as the overall performance of the mixer, e.g., pressure drop across the mixer, is the subject of the study, all the RANS turbulence models considered here provide accurate results; for such a case, the $k-\varepsilon$ model can the model of choice, because of its low computational costs.

However, turbulence models that are dependent on different ways of wall treatments are not able to capture the details of the flow field and misjudge the degree mixing provided by the static mixer. In order to study the performance of the static mixer in a great detail, the $k-\omega$ has more accuracy to capture some of the main aspects of mixing flows.

Comparison of the results obtained by the LES model to those of the RANS models, shows that the $k-\omega$ and RSM models are able to capture some of the main aspects of mixing flows, while they underestimate the mixer performance to 
different degrees. However, the LES model provides the most comprehensive predictive capability of all methods investigated.

Flow parameters, such as static pressure and velocity, were computed. Their mean and instantaneous values were used for determining static mixer parameters, which are not extractable from experiments.

Study of the RTD for the flow across the mixer showed a similar history for a large portion of fluid particles in the flow.

Significant increases in PDU values observed at the end of each even numbered mixing element. This can be explained by considering the fact that mean vorticity magnitudes are larger at those elements.

Instantaneous distribution of fluid particles at flow cross-sections observed at different physical times showed a pattern similar to that obtained by mean flow field parameters.

- Heat transfer rate in a helical static mixer was numerically investigated. Study of heat transfer in a helical static mixer shows that static mixers can decrease the temperature gradient in fluid elements and produce a more uniform temperature distribution within the fluid; however, the mixer's effect on the uniformity of the temperature distribution is not significant, when the difference between the wall temperature and the inlet fluid is small.

A helical static mixer gives a heat flux ratio of at least 2.2 to 2.7 for a noncreeping laminar flow. Heat flux rate is not improved by using the helical static mixer in very low Reynolds number flows. For Reynolds numbers of 10 to 1000 , 
use of helical static mixer increases the heat flux to the flow significantly. The static mixer studied here shows high performance for Reynolds numbers of the order of $10^{2}$. 


\section{FUTURE WORK}

The research presented in this dissertation lays the ground for extensions in several aspects. The following presents a list of potential directions to further proceed.

a. Numerical simulation and performance study of other types of static mixers.

b. Study of alternative helical static mixer geometries in order to optimum the mixer performance.

c. Study of helical static mixer performance using other kinds of nonNewtonian fluids, e.g., shear thickening materials.

d. Performance study of a helical static mixer under two/three-phase flow conditions, e.g., with solid particles entering into the working fluid.

e. Study of heat transfer rate in a helical static mixer under turbulent flow conditions. 